\title{
Equilibrium and Non-equilibrium Emission of Complex Fragments
}

\author{
by \\ David Raymond Bowman \\ Ph.D. Thesis \\ August, 1989 \\ Department of Chemistry \\ University of California, Berkeley \\ and \\ Nuclear Science Division \\ Lawrence Berkeley Laboratory \\ 1 Cyclotron Road \\ Berkeley, CA 94720
}

This work was supported by the Director, Office of Energy Research, Division of Nuclear Physics of the Office of High Energy and Nuclear Physics of the U.S. Deparment of Energy under Contract DE-AC03-76SF00098. 


\title{
EQUILIBRIUM AND NON-EQUILIBRIUM EMISSION \\ OF COMPLEX FRAGMENTS
}

by

David Raymond Bowman

\begin{abstract}
Complex fragment emission $(Z>2)$ has been studied in the reactions of 50,80 , and $100 \mathrm{MeV} / \mathrm{u}{ }^{139} \mathrm{La}+{ }^{12} \mathrm{C}$, and $80 \mathrm{MeV} / \mathrm{u}{ }^{139} \mathrm{La}+{ }^{27} \mathrm{Al}$, ${ }^{2 a} \mathrm{Cu}$, and ${ }^{197} \mathrm{Au}$. Charge, angle, and energy distributions were measured inclusively and in coincidence with other complex fragments, and were used to extract the source rapidities, velocity distributions, and cross sections. The experimental emission velocity distributions, charge loss distributions, and cross sections have been compared with calculations based on statistical compound nucleus decay.

The binary signature of the coincidence events and the sharpness of the velocity distributions illustrate the primarily 2 -body nature of the ${ }^{139} \mathrm{La}+{ }^{12} \mathrm{C}$ reaction mechanism between 50 and $100 \mathrm{MeV} / \mathrm{u}$. The emission velocities, angular distributions, and absolute cross sections of fragments of $20 \leq Z \leq 35$ at $50 \mathrm{MeV} / \mathrm{u}, 19 \leq \mathrm{Z} \leq 28$ at $80 \mathrm{MeV} / \mathrm{u}$, and 17 $\leq \mathrm{Z} \leq 21$ at $100 \mathrm{MeV} / \mathrm{u}$ indicate that these fragments arise solely from the binary decay of compound nuclei formed in incomplete fusion reactions in which the ${ }^{139} \mathrm{La}$ projectile picks up about one-half of the ${ }^{12} \mathrm{C}$ target. At 80 and $100 \mathrm{MeV} / \mathrm{u}$, statistical model calculations are also able to reproduce the isotropic portions of the cross sections for lighter and heavier fragments. However, significant fractions of the total cross sections for these fragments are due to non-equilibrium emission. While the emission process is still mainly binary, the anisotropic angular distributions and the magnitudes of the absolute yields are incompatible with exclusively statistical decay.
\end{abstract}

In the $80 \mathrm{MeV} / \mathrm{u}{ }^{139} \mathrm{La}+{ }^{27} \mathrm{Al},{ }^{n a} \mathrm{Cu}$, and ${ }^{197} \mathrm{Au}$ reactions, the disappearance of the 
binary signature in the total charge and velocity distributions suggests an increase in the complex fragnent and light charged particle multiplicity with increasing target mass. As in the 80 and $100 \mathrm{MeV} / \mathrm{u}^{139} \mathrm{La}+{ }^{12} \mathrm{C}$ reactions, the lighter complex fragments exhibit anisotropic angular distributions and cross sections that are too large to be explained exclusively by statistical emission. Moreover, the cross sections for the heavier fragments with isotropic angular distributions are several times larger than predicted by compound nucleus decay. In addition, the dependence of the coincidence charge distributions $\left(Z_{1}+\right.$ $\mathrm{Z}_{2}$ ), of the velocity distributions, and of the cross sections upon the target mass, as contrasted with the independence of the average source rapidity, suggests that the incomplete fusion mechanism is not applicable to the $80 \mathrm{MeV} / \mathrm{u} 139 \mathrm{La}+{ }^{n 2} \mathrm{Cu}$ and ${ }^{139} \mathrm{La}$ $+{ }^{197}$ Au reactions. 


\section{ACKNOWLEDGEMENTS}

The skills that I have acquired in graduate school, to a large extent, have been gained through the examples of those around me. I have been associated with many excellent scientists, each of whom is outstanding in his or her own way. Four of these people deserve special recognition.

1 am grateful to my thesis advisor, Professor Luciano Moretto, for allowing me access to his deep well of knowledge, for the professional opportunities I have had as a member of his group, for his example of what a scientist should strive to be, and for his generally good nature.

Di. Gordon Wozniak deserves a special acknowledgement for illustrating the traits of an experimentalist par excellence: patience, thoroughness, and perseverance; and for being a good fellow even though he was the boss.

As a postdoc, Dr. Bob Charity had the unenviable day-to-day task of training a green graduate student. I have benefited immensely from his extensive knowledge of both experimental and theoretical nuclear physics, and I am grateful to him for his willingness to share his knowledge, his patience with my questions, his pleasant demeanor, and also for supplying tinnies and lamingtons.

I am indebted to soon-to-be Dr. Walter Kehoe for top-notch experimental help, and for transferring to me some of his unmatched mechanical, organizational, and interpersonal skills, although none of his culinary skill.

In addition, I would like to mention my senior collaborators who have helped me along in various ways: Dr. Peggy McMahan, Dr. Dick McDonald, Professor Alice Mignerey, Professor Ileana Iori and Dr. Arialdo Moroni. Thanks also go to Dr. Graham Peaslee, Dr. Nicola Colonna, Mr. Dimitri Delis, and Mr. Karl Hanold for assistance in various phases of my thesis project, including experimental help, animated "discussions", and fierce ping-pong matches. 
Last, but not least, I would like to acknowledge my wife June for her love and support during my years as a student, and my parents for the many things that they have done for me throughout my life. 


\section{EQUILIBRIUM AND NON-EQUILIBRIUM EMISSION OF COMPLEX FRAGMENTS}

\section{TABLE OF CONTENTS}

I. Introduction. $\ldots \ldots \ldots \ldots \ldots \ldots \ldots \ldots \ldots \ldots \ldots \ldots$

A. Historical Survey of Complex Fragment Emission . . . . . . . 1

B. Theoretical Background. . . . . . . . . . . . 3

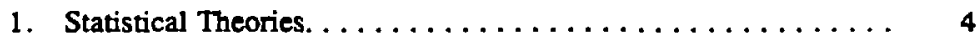

a) Compound Statistical Emission of Complex Fragments. ..... 4

b) Nuclear Shattering. . . . . . . . 7

c) Chemical Equilibrium Theories. ............. 9

d) Percolation Theories. . . . . . . . . . . . 10

2. Dynamical Models. . . . . . . . . . . . . . . 10

C. Experimental Features of Complex Fragment Emission at E/A $\leq 50 \mathrm{MeV}$. 12

1. The Anisotropic Source. ................ 12

2. The Isotropic Source - Compound Statistical Decay. . . . . . . 13

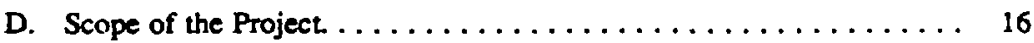

Figures. ........................ 18

II. Experimental. . . . . . . . . . . . 20

A. Choice of Projectile and Target. $\ldots \ldots \ldots \ldots \ldots \ldots \ldots \ldots$

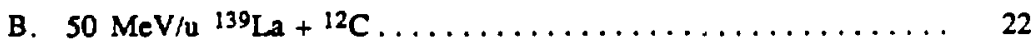

1. Beam, Targets, and Detection Hardware. . . . . . . . 22

2. Electronics and Logic. . . . . . . . . . . . . . 24

3. Calibrations and Analysis. ............... 25

C. 80 and $100 \mathrm{MeV} / \mathrm{u}{ }^{139} \mathrm{La}+{ }^{12} \mathrm{C},{ }^{27} \mathrm{Al}$, nat $\mathrm{Cu}$, and ${ }^{197} \mathrm{Au} \ldots \ldots \ldots 28$ 
1. Beam, Targets, and Detection Hardware. . . . . . . 28

2. Electronics and Logic. ................ 30

3. Calibrations and Analysis. . . . . . . . . . 31

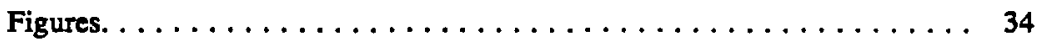

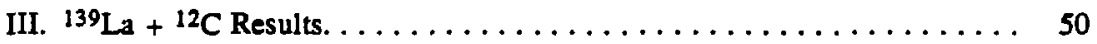

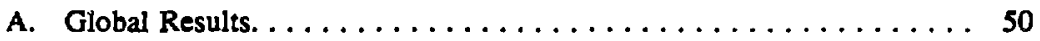

1. Two-fold Coincidence Events. .............. 50

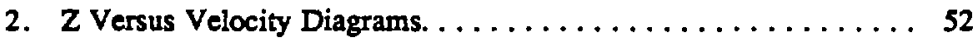

3. $v_{\|}-v_{\perp}$ Plots. $\ldots \ldots \ldots \ldots \ldots \ldots \ldots \ldots \ldots \ldots \ldots \ldots \ldots$

B. Quantitative Results. ................... 56

1. Source Rapidities. .................. 56

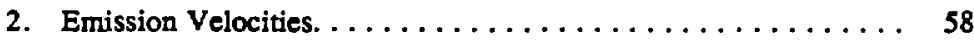

3. Angular Distributions. ................. 61

4. Cross Sections. . . . . . . . . . . . . . . 64

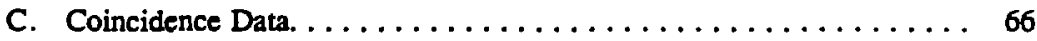

1. Total Charge and Rapidity Dependence on Fragment Multiplicity.... 66

2. Charge Loss and Widths. . . . . . . . . . . . 66

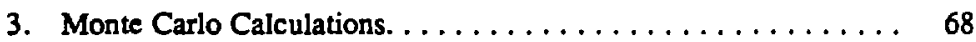

D. Model Calculations. . . . . . . . . . . . . . . . . . . . 69

1. Incomplete Fusion. . . . . . . . . . . 70

2. Statistical Decay Calculations. . . . . . . . . . 72

Tables. . . . . . . . . . . . . .

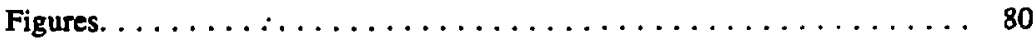

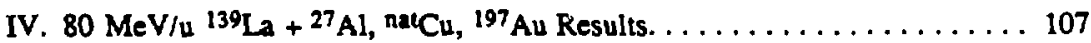

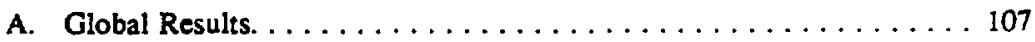

1. Coincidence Charge and Rapidity Distributions. ........ 107

2. Z Versus Velocity Plots. . . . . . . . . . . . . 109 
3. $\mathrm{Y}-\mathrm{P}_{\perp} / \mathrm{mc}$ Plots. $\ldots \ldots \ldots \ldots \ldots \ldots \ldots \ldots \ldots \ldots \ldots \ldots$

B. Quantitative Results. . . . . . . . . . . . . . . 112

1. Source Rapidities. . . . . . . . . . . . . . . . 112

2. Emission Velocities. ................... 114

3. Angular Distributions. .................. 117

4. Cross Sections. . . . . . . . . . . . . . . 117

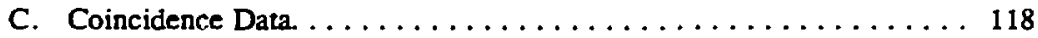

D. Model Calculations. . . . . . . . . . . . . . . . 121

Table. . . . . . . . . . . . . . . . 122

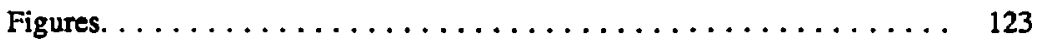

V. Summary and Conclusions. . . . . . . . . . . . . . 146

A. $50 \mathrm{MeV} / \mathrm{u}{ }^{139} \mathrm{La}+{ }^{12} \mathrm{C} \ldots \ldots \ldots \ldots \ldots \ldots \ldots \ldots \ldots \ldots$

B. 80 and $100 \mathrm{MeV} / \mathrm{u}{ }^{139} \mathrm{La}+{ }^{12} \mathrm{C} \ldots \ldots \ldots \ldots \ldots \ldots \ldots 7$

C. $80 \mathrm{MeV} / \mathrm{u}{ }^{139} \mathrm{La}+{ }^{27} \mathrm{Al}$, nat $\mathrm{Cu}$, and ${ }^{197} \mathrm{Au} \ldots \ldots \ldots \ldots \ldots 148$

Appendix I: Detector Efficiency and Kinematics. . . . . . . . . . 150

A. Detector Efficiency. . . . . . . . . . . . . . 150

B. Kinematics. . . . . . . . . . . . . . . . . 151

1. $50 \mathrm{MeV} / \mathrm{u}{ }^{139} \mathrm{La}+{ }^{12} \mathrm{C} \ldots \ldots \ldots \ldots \ldots \ldots \ldots \ldots \ldots 151$

2. 80 and $100 \mathrm{MeV} / \mathrm{u}{ }^{139} \mathrm{La}+{ }^{12} \mathrm{C},{ }^{27} \mathrm{Al}$, nat $\mathrm{Cu}$ and ${ }^{197} \mathrm{Au} \ldots \ldots \ldots 152$

Appendix II: An Incomplete Fusion Model. . . . . . . . . . . . 160

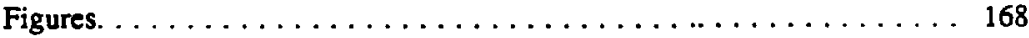

Footnotes. . . . . . . . . . . . . . . . . . . . 173

References. . . . . . . . . . . . . . . . . . . . . . . 179 


\section{INTRODUCTION}

\section{A. Historical Survey of Complex Eragment Emission}

Historically, products from compound nucleus decay have been divided into two distinct classes: light particles, such as neutrons, protons, and alpha particles; and, from heavier systems, fission fragments with masses approximately half that of the fissioning nucleus. Although both classes of products are emitted statistically by compound nuclei, their vast difference in mass gave rise to two different statistical theories: a theory based upon the detailed balance principle for the evaporation of light particles [We 37], and a theory based upon the transition state method for fission [Bo 39]. This theoretical dichotomy was due, in no small part, to the wide gulf in the product mass distributions between alpha particles and fission fragments.

Fragments of intermediate mass, commonly termed complex fragments $(C F)$, were first observed radiochemically following high energy proton $(2.2 \mathrm{GeV})-$ nucleus collisions in the 1950's [Mi 53, Su 54, Wo 56]. Subsequent counter experiments were performed at the Lawrence Berkeley Laboratory Bevatron and BEVALAC with very energetic proton and later light ion beams through the 1970's and into the 80's [Po 71, Hy 71, Ko 73, Ze 75, Go 77, We 78, Me 80, Wa 82, Gu 82, Wa 83].

These observations of novel nuclear decay products, unseen at lowez energy, were taken as evidence of a new reaction mechanism at higher bombarding energy'. The high energy reactions were assumed to proceed through a fast nucleon cascade step, followed by fragment emission from a moving source. The fragment energy spectra at various laboratory angles were fit to Maxwell distributions in a frame moving at a fixed laboratory velocity [Po 71]. The velocity of the moving source, and the slope of the high energy tail of the Maxwell distribution ( $\tau$-parameter), which in a thermal interpretation is equivalent to the temperature of the emitting system, were extracted from the fits.

Empirical treatments such as these successfully fit the experimental energy spectra 
of light-ion induced reactions at $\mathrm{E} / \mathrm{A} \geq 100 \mathrm{MeV}$ [Go 77], especially when more than one moving source was considered [We 78]. The extracted source velocities and $\tau$-parameters were essentially independent of fragment mass [We 78]. However, the analysis of this relatively small body of work shed little light on the complex fragment formation mechanism.

With the discovery of deep-inelastic reactions in the 1970's, fragments of virtually all masses were detected at low bombarding energy [Sc 77, Mo 81a]. (For purpose of discussion, bombarding energies $\mathrm{E} / \mathrm{A}$ of $<10 \mathrm{MeV}$ will be termed "low energy", from 10 $100 \mathrm{MeV}$ will be termed "intermediate energy", and above $100 \mathrm{MeV}$ will be termed "high energy".) The products from these reactions were emitted in binary processes; the two primary reaction produets could then de-excite by light particle emission, or for heavier primary fragments, by sequential fission. Various degrees of energy relaxation running the gamut from quasi-elastic scattering to completely damped reactions were observed. The mass distributions were very broad; fragments of all masses up to the heavier entrance channel reaction partner were produced. While generally not completely relaxed, the mass distributions showed sensitivity to the potential energy of the dinuclear system. A variety of angular distributions was also observed. Depending upon the amount of energy damping and the mass of the fragment, the angular distributions could be forward-peaked (for projectiie-like fragments), backward peaked (for target-like fragments), side-peaked, or in the case of complete relaxation, flat ${ }^{1}$. In general, the binary products from low energy deep-inelastic reactions could be identified as projectile-like and target-like.

In the 1980's heavy-ion beams became available in the previously unexplored intermediate energy region. The first systematic investigations showed that fragments of 3 $\leq Z_{\text {frag }} \leq 20$ had monotonically decreasing charge distributions that could be described in terms of a power law $\mathrm{P}(\mathrm{Z}) \propto \mathrm{Z}^{-2.6}[\mathrm{Ly}$ 82, Ch 83a, Ja 83a, So 83, Fi 84]. The angular distributions were forward peaked for the lightest complex fragments, smoothly changing into forward/backward symmetric distributions with increasing Z-value [So 83, Ch 83a, Fi 
84]. The energy spectra of the light complex fragments were Maxwellian ai forward angles, gradually evolving into Gaussians at backward angles. The heavier complex fragments had Gaussian energy distributions at all laboratory angles [So 83, Fi 84].

The large anisotropy of the complex fragment angular distributions and the strong angular dependence of their energy spectra illustrated that, unlike at high energy, moving sources with velocities and $\tau$-parameters independent of fragment charge could not fit the experimental energy spectra. Jacak et al. [Ja 83a] claimed that the fragments $1 \leq A \leq 14$ in the reactions 42,92 , and $137 \mathrm{MeV}, \mathrm{u}^{40} \mathrm{Ar}+{ }^{197} \mathrm{Au}$ were emitted from a common moving source. However, later studies have shown dependences of both the source velocity and $\tau$ parameter upon the fragment charge [Fi 84, Tr 85], with the lighter fragments being characterized by faster sources and larger $\tau$-parameters.

The novel complex fragment ernission process was hailed as the onset of a new and exciting reaction mechanism, and, almost overnight, spawned a flurry of theoretical activity.

B. Theoretical Backoround

Each of the theories proposed to account for the rapid increase in complex fragment emission above bombarding energies of approximately $10 \mathrm{MeV} / \mathrm{u}$ can be classified as either statistical or dynamical.

Fully statistical decay models assume a decoupling of the interaction and decay processes. Following the projectile - target interaction the source of the fragments, be it the entire projectile - target system or only some portion, is assumed to attain equilibrium prior to the emission process. The only reievant parameters in these theories are the conserved quantities of the reaction - the total mass, the total charge, the excitation energy, and the angular momentum.

Dynamical theories, on the cther hand, depend in principle upon a much larger set of variables and require the knowledge of collestive quartitics like inertias, viscosities, etc., which are not precisely known. The two venerabie statistical decay models mentioned 
above, the evaporation formalism of Weisskopf [We 37] and the transition state (fission) theory of Bohr and Wheeler [Bo 39], have been very successfully applied to nuclear reaction theory. Statistical theories have also been applied to certain aspects of dynamical processes with success. Thus it is not surprising that statistical theories of complex fragment emission sprung up first.

1. Statistical Theories

a) Compound Statistical Emission of Complex Fragments

In their present formulation compound statistical emission theories differ from other statistical theories in that they predict only binary decays. The emission of a number of fragments is described as a sequential mechanism, with the compound nucleus relaxing and reattaining equilibrium prior to each subsequent decay. This is a well-known occurence at low energy. A very fissile nucleus may emit one or more neutrons prior to fission. Second- and higher-order chance fission is still well described in terms of compound nucleus decay by taking into account the excitation energy and angular momentum removed by the emitred neutron(s).

The transition state model of complex fraginent emission, as developed by Moretto [Mo 72, Mo 75], generalizes the method of Bohr and Wheeler [Bo 39, Wh 63] which has been successfully applied to fission reactions for many years. The saddle point of Bohr and Wheeler was extended to a ridge line of $Z$-dependent conditional saddles using the charge asymmetry coordinate, $\mathrm{Z}_{2 s y}=\mathrm{Z}_{\mathrm{frag}} / \mathrm{Z}_{\mathrm{CN}}$, which is the ratio of the emitted fragment charge to the compound nucleus charge. In this way, the model does away with the artificial distinction between evaporated particles and fission fragments in a natural manner.

In analogy with fission theory, the decay width at any conditional saddle is proportional to the number of states above the conditional barrier, and can be written as:

$$
\Gamma_{Z}=\frac{1}{2 \pi \rho_{0}\left(U_{0}\right)} \int_{0}^{E^{*} \cdot E_{s a d}(J)} \rho_{s a d}\left(U_{s a d}\right) d \varepsilon .
$$


Here $\rho_{0}$ is the level density of the compound nucleus with thermal energy $U_{0}=E^{*}-E_{0}(J)$, equal to the excitation energy minus the rotational energy at angular momentum $J$, and $\rho_{\mathrm{sad}}$ is the level density at the conditional saddle with thermal energy $U_{s a d}=E^{*}-E_{s a d}(J, Z)-E$. $E_{\text {sad }}$ is the deformation plus rotational energy at angular monconium $J$ of the saddle point configuration, and $\varepsilon$ is the kinetic energy along the fission mode. It should be noted that the decay widths determine the charge distributions aiong the ridge line. The asymptotic charge distributions can be influenced by mass transfer in the descent from the ridge line to the scission points. The effect will generally be smaller for lighter nuclei $(A<100)$, where the saddle and scission points are nearly degenerate, than for heavier nuclei ( $A>200$ ), where the saddle point shapes are fairly compact. However, for very asymmetric decay of heavy nuclei, the saddle and scission points are also nearly degenerate, so the asymmetric products are strongly correlated with their respective decay widths [Sa 89].

Equation (i) can be simplified by first expanding the logarithm of $\rho_{\text {sad }}$ about $\varepsilon=0$ to yieid:

$$
\Gamma_{Z} \equiv \frac{\rho_{\mathrm{sad}}\left[\mathrm{E}^{*}-\mathrm{E}_{\mathrm{sad}}(\mathrm{J}, \mathrm{Z})\right]}{2 \pi \rho_{\mathrm{o}}\left[\mathrm{E}^{*}-\mathrm{E}_{\mathrm{o}}(\mathrm{J}, \mathrm{Z})\right]} \int_{0}^{\mathrm{E}^{*}-\mathrm{E}_{\mathrm{sad}}(\mathrm{J})} e^{-\varepsilon / T} \mathrm{d \varepsilon},
$$

remembering that $\frac{\partial \ln p}{\partial U} \equiv \frac{1}{T}$. The nuclear temperature is $T \equiv \sqrt{\frac{U}{a}}$, where $a$ is the nuclear level density parameter, usually taken to be in the range of $\mathrm{A} / 10$ to $\mathrm{A} / 8$. The above integral can be evaluated between the limits of 0 and $\infty$ :

$$
\Gamma_{Z} \equiv \frac{T}{2 \pi \rho_{0}\left[E^{*}-E_{o}(J)\right]} \rho_{\text {sad }}\left[E^{*}-E_{s a d}(J, Z)\right] .
$$


In order to recover the Bohr-Wheeler fission decay width we must perform an integration over all of the conditional saddle points that contribute to fission. For heavier nuclei we can assume a parabolic shape for the saddle point energies near symmetry (see Figure I.1) of $E_{\text {sad }}(J, Z) \equiv E_{\text {fiss }}(J)+b Z^{\prime 2}$, where $E_{\text {fiss }}(J)$ is the conditional saddle point energ;' at symmetry and $Z^{\prime}=Z-Z_{\text {sym- }}$ Expanding the logarithm of $\rho_{\text {sad }}$ about $Z^{\prime}=0$ yields:

$$
\Gamma_{F} \equiv \frac{T \rho_{\text {sad }}\left[E^{*}-E_{f i s s}(J)\right]}{2 \pi \rho_{0}\left[E^{*}-E_{0}(J)\right]} \int e^{-b Z^{\prime 2} / T} d Z^{\prime}
$$

After integrating between $-\infty$ to $\infty$ we have:

$$
\Gamma_{F} \equiv \frac{T \rho_{s a d}\left[E^{*}-E_{f i s s}(J)\right]}{2 \pi \rho_{0}\left[E^{*}-E_{0}(J)\right]} \sqrt{\frac{\pi T}{b}} \equiv \frac{T \rho_{\text {fiss }}\left[E^{*}-E_{f i s s}(J)\right]}{2 \pi \rho_{0}\left[E^{*}-E_{0}(J)\right]},
$$

where we have defined $\rho_{f i s s}\left[E^{*}-E_{f i s s}(J)\right]=\sqrt{\frac{\pi T}{b}} \rho_{s a d}\left[E^{*}-E_{f i s s}(J)\right]$ as the level density for fission. This shows the relationship between the fission and the individual complex fragment decay widths.

If instead we explicitly define a barrier for each conditional saddle point $B_{Z}=E_{s a d}$ $(J, Z)-E_{0}(J)$, in the limit of high excitation we can expand the logarithm of $p_{: a d}$ about $B_{Z}$ $=0$ in (3) to obtain:

$$
\Gamma_{Z} \equiv \frac{T}{2 \pi} \frac{\rho_{\text {sad }}\left[E^{*}-E_{o}(J)\right]}{P_{o}\left[E^{*}-E_{o}(J)\right]} e^{-B_{Z} T} \equiv \frac{T}{2 \pi} e^{-B_{Z} / T}
$$

because at high excitation $\rho_{\text {sad }}\left[E^{*}-E_{o}(J)\right] \equiv \rho_{o}\left[E^{*}-E_{o}(J)\right]$. 
Figure I.1 shows the conditional barriers for complex fragment emission calculated using the Liquid Drop Model [Da 85] from a ${ }^{139} \mathrm{La}$ nucleus with $\mathrm{J}=0$, and a qualitative calculation of the corresponding yields [from eq. (6)] at excitation energies of 75,170 , and $300 \mathrm{MeV}$. At low excitation energy, only light particles, evaporation residues, and, from heavier nuclei, fission fragments have cross sections larger than typical detection thresholds. The observed gulf in the product mass distributions between alpha particles and fission fragments gave rise to the widespread belief that fission and evaporation were distinct processes. Because of the exponential dependence of the yields upon $e^{-B} z^{T}$, the yields increase dramatically and the mass distribution becomes much flatter with increasing excitation energy. Hence, in the intermediate energy regime, fragments of all mass asymmetries are detectable, and the continuity of the emission process as a function mass asymmetry is observable.

Compound statistical models of complex fragment emission have also been developed using the detailed balance formalism. These theories calculate the decay probabilities $P_{A B}$ based on the phase space density of the compound nucleus $P_{A}$ and the asymptotic products $\rho_{B}$, and on the inverse cross section $\sigma_{B A}$ [Fr 83a, Fr83b, Go 88]:

$$
\mathbf{P}_{\mathrm{AB}} \propto \sigma_{\mathrm{BA}} \frac{\rho_{\mathrm{A}}}{P_{\mathrm{B}}} .
$$

Typically the optical model is used to calculate the inverse cross section for the absorption of the emitted fragment. These calculations are fairly accurate for the absorption of light particles (as in the Weisskopf evaporation formalism), where the heavy fragment remains nearly spherical. However, for complex fragment emission the two reaction partners are strongly polarized during the decay process, and corrections must be made by introducing unrealistic strong absorption radii and barrier transmission coefficients.

\section{b) Nuclear Shattering}

Using information theory, a method was proposed by Aichelin and Huefner to calculate the probability of fragment formation as a function of charge [Ai 84a, Ai84b]. The 
method was later shown to be a saddle point approximation to the Euler number partition [So 85]. If $P(m, Z)$ is the probability of producing a fragment of charge $Z$ with multiplicity $\mathrm{m}$ from an initial nucleus of charge $\mathrm{Z}_{0}$, then normalization of the probability and conservation of charge lead to the following constraints:

$$
\begin{aligned}
& \sum_{m} P(m, Z)=1 \\
& \sum_{m} \sum_{Z=1}^{Z_{0}} m Z P(m, Z)=Z_{0} .
\end{aligned}
$$

The form of $P(m, Z)$ can be derived from the principle of maximum likelihood or minimum bias. Using Lagrange multipliers to introduce the above constraints, $P(m, Z)$ was found to be:

$$
P(m, Z) \equiv\left[\exp \left(\frac{1.28 Z}{\sqrt{Z_{0}}}\right)-1\right]^{-1}
$$

Since all partitions are equally likely, the only parameter in the model is the charge of the fragmenting nucleus $\left(Z_{0}\right)$. This model yields different results if, instead of partitioning the charge, the charge distribution is obtained by partitioning the mass and then scaling the distribution according to the initial charge to mass ratio [So 85]:

$$
P\left(m, \frac{A Z_{0}}{A_{0}}\right) \equiv \frac{Z_{0}}{A_{0}}\left[\exp \left(\frac{1.28 A}{\sqrt{A_{0}}}\right)-1\right]^{-1} \neq P(m, Z)
$$

This model has not received much serious attention in the intermediate energy domain, where mean field effects are very important. 
c) Chemical Equilibrium Theories

A hypothesis that has received much more attention is that of a phase transition from the normal nuclear fluid to a vapor of nucleons. Since the nucleon - nucleon interaction is composed of a very short range repulsive core and a short range attractive part, which is similar to that between atoms in a monatomic Van der Waals gas, it was realized that nuclear matter should be able to undergo a liquid to vapor transition. This behavior was first proposed by Sauer et al. in 1976 [Sa 76]. Following the interpretation of data from high energy proton-induced reactions with this model [Fi 82, Mi 82, Hi 84], there was a rebirth of theoretical interest. Several different versions of this theory have been expounded [ $\mathrm{Ja} 83 \mathrm{~b}, \mathrm{Ja} \mathrm{84,} \mathrm{Cu} 83, \mathrm{Si} 84, \mathrm{Lo} \mathrm{84,} \mathrm{Pa} \mathrm{84,} \mathrm{Pa} \mathrm{85],} \mathrm{all} \mathrm{based} \mathrm{on} \mathrm{the}$ Fischer model of droplet formation [Fi 67, Fi 71].

The probability of finding a cluster of A particles in a fluid is given by:

$$
P_{A}=e^{A\left(\mu-\mu_{L}\right) / T} e^{-c A^{2 / 3} / T} P_{0} A^{-\tau}
$$

where $\mu$ is the chemical potential of clusters of size one, $\mu_{\mathrm{L}}$ is the chemical potential of the liquid, $T$ is the temperature, $c$ is the surface energy coefficient, and $\tau$ is a critical exponent. The first factor in the above equation is related to the volume energy of a cluster, and the second factor to the surface energy. The third factor arises from the statistical weight of a cluster of size $\mathrm{A}$. This factor only contributes when the first two exponential factors are approximately equal to one.

When $\mu_{L}>\mu$ the system is in the gas phase and the cluster probability falls exponentially with A. On the other hand, when $\mu_{\mathrm{L}}<\mu$ the system is in the liquid phase. When $\mu=\mu_{\mathrm{L}}$ the the liquid and gas phases are in equilibrium and the probabilities are determined by the surface energy.

At the critical temperature, which is the highest temperature at which the two phases can exist in equilibrium, the densities of the liquid and gas are equal and $c=0$. At this 
point the liquid-to-vapor transition is second order. The distribution of sizes is determined by the third factor in equation (12), and the probability distribution assumes the form of a power law, $P(A) \propto A-\tau$.

Of course the finite size of nuclei, the Coulomb interaction, and the shell structure of the nucleus are all ignored in the above model. This calls into question the applicability of the liquid-vapor equilibrium theory to nuclear systems.

Other chemical equilibrium models have been proposed that do incorporate the above factors. In these theories the decay configuration is assumed to have a number of distinct "pre-fragments" existing within a somewhat arbitrary "freeze-out" volume. This is a crucial assumption because the potential energy of these configurations determines the decay rates, and it is also the facet most open to criticism because it has not been accepted that an equilibrated nucleus ever approaches such configurations. Multifragment branching ratios have been calculated using the microcanonical ensemble [ $\mathrm{Ab} 86, \mathrm{Gr} 86, \mathrm{Gr} 87 \mathrm{a}$, Zh 87a, Zh 87b], canonical ensemble [Bo 85a, Bo 855, Ba86a], and granu caisonical ensemble [Ra 81] formalisms.

d) Percolation Theories

These theories treat the nucleus as a three dimensional lattice of nucleons connected by bonds. The bond breaking probabilities are related to the excitation energy of the nucleus. With increasing excitation energy (fewer existing bonds) the system evolves from consisting of mainly one large fragment to many smaller fragments. This behavior is similar to that described above for the liquid - vapor phase transition, and in fact, percolation models have been used to simulate such transitions for finite systems [Ba 85 , Ba 86b, Ne 86a, De 87a, De 87b]. Pre-equilibrium models have been used in conjunction with these theories to determine input parameters such as the lattice site occupation and bond breaking probabilities [Ne $86 \mathrm{~b}, \mathrm{Ce} 88]$ ].

2. Dynamicai Models

In recent years several dynamical models (Büitzmann-Ueling-Uhlenbeck (BUU) 
[Be 84, Ai 85, $\mathrm{Kr}$ 85, Mo 85, Ai 86a], or Landau-Vlasov (LV) [Gr 85, Gr 87b, Gr 87c], among others) have sprouted that treat nuclear interactions semi-classically by using the Vlasov equation to determine the time evolution of the phase space distribution function $f(r, p, t):$

$$
\frac{\partial f}{\partial t}+\frac{P}{m} \cdot \nabla_{r} f-\nabla_{r} U_{r} \cdot \nabla_{p} f=I_{\text {coll }}[f]
$$

where $U_{r}$ is the mean field potential. To the classical Vlasov equation a collision term ( $\left.I_{\text {coll }}[f]\right)$ has been added that simulates Pauli-blocked particle - particle scattering. The numerical solution to the above equation is found as the motion in phase space of many Gaussian wave packets ( $\sim 50$ per nucleon) that are chosen randomly from the initial phase space distribution function.

The application of dynamical models to the problem of CF emission appears to be a promising avenue of research due to the increased importance of dynamical processes in the intermediate energy regime. However, these models have some serious drawbacks. The mean-field aspect of the models does not allow for fluctuations or the emission of clusters of nucleons from the excited regions of the system. Thus it is impossible to make compar.sons with experimental distributions. To circumvent this difficulty, a dynamical model simulating the pre-equilibrium phase of the reaction has recently been combined with a statistical multifragmentation model that simulates the decay of the excited system [Sn 88].

The most recent development in this very active field has been the introduction of the Quantum Molecular Dynamics (QMD) model [Ai 86b, Ai 87]. In this approach, the Gaussian wave packets interact by mutual 2- and 3-body forces. The great advantage of this approach over the standard BUU and LV theories is that correlations and fluctuations are preserved, hence the fragment emission process can be studied theoretically. In 
principle distributions can be generated and compared with experimental data, although the computer-intensiveness of this model is a serious drawback.

Virtually all of the above models claimed agreement with the cross sections and inclusive charge distributions measured in the first intermediate energy experiments. Thus much more detailed experimental work was needed to adequately test the proposed models C. Experimental Features of Complex Fragment Emissionat E/A $\leq 50 \mathrm{MeV}$

Attempts to fit the energy spectra of complex fragments as a function of both angle and charge have required the introduction of three or more sources (projectile-like, targetlike, and intermediate rapidity, plus others). The projectile-like source is usually attributed to a direct reaction process, such as quasi-elasic scattering, and will not be discussed here in further detail. The other two sources, intermediate rapidity and target-like, have been respectively associated with the anisotropic and isotropic porticns of the angular distributions.

\section{The Anisotropic Source}

Recent studies have shown that the anisotropic light complex fragment emission grows in importance relative to isotropic emission as the bombarding energy is increased from $20-50 \mathrm{MeV} / \mathrm{u}$ [Fi 89]. What is the productir. mechanism for these anisotropic fragments? It has been proposed that they are produced by a mechanism that is very similar to low energy deep-inelastic scattering [Bo 88].

Borderie et al [Bo 88] have shown that most of these fragments are emitted in binary processes, and that the fragments are formed with both complete and incomplete energy relaxation over a large angular region. These results are similar to those obtained for the same system in the low energy regime [Ga 75], and interpreted as classical deepinelastic scattering. Other studies have confirmed the projectile- and target-like character of the exit channel fragments [Ch 88a, Ch 88b, Ha 89, Pl 89].

The polarization of gamma rays in coincidence with non-equilibrium CF has illustrated another dynamical aspect of the process. The fragments are preferentially 
deflected to negative scattering angles by the nuclear mean field [Ts 88 ]. These polarizations were in agreement with those mensured for heavy fragments detected near the grazing angle in deep-inelastic collisions at lower energy [Du 84, Tr 84]. Purely dynamical calculations, not incorporating statistical fluctuations, over-predicted the measured polarizations, illustrating the partially relaxed nature of the mechanism [Ts 88], in agreement with the low energy deep-inelastic scattering process.

Arguments for altemative interpretations of this mechanism have been proposed, based on perceived differences between the process at intermediate energy and the deepinelastic process at low energy [Fi 84]. The differences may be due to dissimilar experimental conditions, such as the entrance channel mass asymmetry and the detector placement relative to the classical grazing angle, rather than to any fundamental difference in mechanism. In fact, studies of the ${ }^{20} \mathrm{Ne}+{ }^{\text {nat }} \mathrm{Ag}$ system at 8.75 and $12.6 \mathrm{MeV} / \mathrm{u}$ [Ba 76] have shown charge and angular distributions that are very similar to those generally observed in asymmetric reactions at intermediate energy.

Of course, as the bomba ling energy is increased, one expects changes in the reaction mechanism, due, for example, to the increased importance of nucleon - nucleon interactions. It seems likely that the anisotropic mechanism is the intermediate energy analog of the classical deep-inelastic scattering process, originating from the same range of impact parameters as its low-energy counterpart.

2. The Isotropic Source - Compound Statistical Decay

The first systematic study of CF emission, in the ${ }^{3} \mathrm{He}+{ }^{\text {nat }} \mathrm{Ag}$ system, demonstrated the compound nucleus nature of the process [So 83, So 84, Mc 85]. The use of the very light ${ }^{3} \mathrm{He}$ projectile eliminated quasi-elastic reactions as a source of complex fragments. Contributions from the anisotropic component were similarly eliminated by measuring the yields at $170^{\circ}$. The excitation functions for the equilibrium emission of fragments of $3 \leq \mathrm{Z} \leq 11$ from $50-135 \mathrm{MeV}$ are shown in Fig. 1.2 [Mc 85]. The lowest excitation energy is only about $10 \mathrm{MeV}$ above the largest conditional barriers, hence the 
cross sections are very small, between $0.05-10$ microbarns for $Z$-values larger that 3 . The subsequent opening of phase space with increasing excitation energy is beautifully illustrated by these excitation functions, which are reminiscent of those for fission [Va 73]. These rapidly increasing cross sections are in excellent agreement with theoretical predictions of the transition state formalism described above (solid lines).

Along with establishing the statistical basis for CF emission, these studies also explained tise pervasiveness of complex fragments in the intermediate energy regime as contrasted to their extreme rarity below $10 \mathrm{MeV} / \mathrm{u}$ bombarding energy. The important quantity in the statistical model is the available energy above the barrier, and it is necessary to deposit a few hundred $\mathrm{MeV}$ of excitation energy into compound nuclei before CFs become easily detectable with cross sections on the order of one mb per Z-value.

This process of CF emission from compound nuclei has been systematically studied for very asymmetric entrance channels throughout the periodic table, from $8 \mathrm{MeV} / \mathrm{u}$, where the process is very rare, up to $40 \mathrm{MeV} / \mathrm{u}$, where the CF multiplicity per event can approach one [So 84, Au 85, Ch 86, Au 87, Ch 88a, Ch 88b, Ha 89, P1 89]. For fragments intermediate in mass between the projectile and the target, the cross sections can be explained as originating solely from the binary decay of equilibrated compound nuclei. This conclusion has been reached from the analysis of the source velocities, emission velocities, angular distributions, direct measurements of the binary nature of the process (as opposed to the multifragment nature of the other proposed processes), and, above all, by the shape of the charge distributions and the magnitude of the cross sections as a function of excitation energy.

The observable change in mechanism in these reactions is that the compound nucleus results from progressively less fusion of target and projectile as the bombarding energy is increased. An interesting question in regard to the dynamics of nucleus - nucleus collisions is the fate of the incomplete fusion process at larger bombarding energies. Thir question will be discussed below. 
Less asymmetric systems such as ${ }^{93} \mathrm{Nb}$ or ${ }^{139} \mathrm{La}+{ }^{27} \mathrm{Al}[\mathrm{Ch} 88 \mathrm{~b}, \mathrm{Ch} 89]$ and ${ }^{139} \mathrm{La}$ $+{ }^{64} \mathrm{Ni}[\mathrm{Co} 89]$ have been studied at bombarding energies of $11-18 \mathrm{MeV} / \mathrm{u}$. Fragments intermediate in mass between the target and the projectile are emitted in binary reactions, with Coulomb velocities from a source corresponding to complete or nearly complete fusion. However it is impossible to reproduce the measured cross sections without considering entrance channel $\mathcal{L}$-waves larger than $\mathrm{J}_{\text {criv }}$, where the symmetric fission barriers vanish. This precludes compound nucleus emission following complete fusion as the source of all of these fragments.

Two explanations that could account for these experimental results are: 1) CF production via a quasi-fission or non-statistical fission process [To 85], or 2) statistical emission of CF following incomplete fusion reactions with entrance channel $L$-waves larger than $J_{\text {crir }}$ In the latter case the orbital angular momentum taken away by the incomplete fusion products could decrease the transferred spin to values smaller than $J_{\text {erit }}$ and thus allow true statistical competition between the various exit channels.

For reactions with very asymmetric entrance channels such as ${ }^{93} \mathrm{Nb}+{ }^{9} \mathrm{Be},{ }^{12} \mathrm{C}$ or ${ }^{139} \mathrm{La}+{ }^{12} \mathrm{C}$ a large range of impact parameters gives rise to complete overlap between the target and the projectile. This should simplify or at least limit the number of competing processes, and in fact it has been shown that the fragments are all emitted by a source with a well-defined velocity. Thus it is fairly easy to characterize the reactions from the inclusive data alone. The study of the more symmetric ${ }^{139} \mathrm{La}+{ }^{64} \mathrm{Ni}$ reaction at $18 \mathrm{MeV} / \mathrm{u}$ [Co 89] has shown much more complicated fragment velocity distributions, with no welldefined source. By gating on specific coincidence source velocities, it was shown that the distributions result from a continuum of sources, each emitting fragments of a given charge with a well-defined velocity. This study indicates that symmetric reactions, where each impact parameter gives rise to a different amount of geometric overlap between target and projectile, can bu virtually impossible to untangle from the sudy of inclusive distributions alone. 


\section{Scope of the Project}

For very asymmetric systems at incident energies of $8-40 \mathrm{MeV} / \mathrm{u}$ and excitation energies per nucleon approaching $4 \mathrm{MeV}$, the compound statistical model has successfully explained the isotropic portion of the complex fragment cross sections following both complete and incomplete fusion reactions. It is always interesting to extend the study of a well-understood process into new domains to determine the limits of the process, and to search for new effects. For example, one such effect that might occur at larger excitation energies is the possible sequential statistical emission of several complex fragments from very hot compound nuclei.

Two natural questions arise that are relevant to the extension of these studies to higher energies:

1) What is the maximum amount of excitation energy or excitation energy per nucleon that a nucleus can thermalize?

2) What is the maximum relative velocity beyond which there is no longer caf " of any portion of the target nucleus by the projectile? That is, when does the incomplete fusion process cease?

Since this second question concerns the dynamics of the interaction, the maximum relative velocity that can sustain incomplete fusion will depend upon the impact parameter and the target - projectile combination. It is to be expected that above some bombarding energy the incomplete fv : on process will disappear. For instance in the ${ }^{139} \mathrm{La}+{ }^{12} \mathrm{C}$ system, predictions from a simple geometrical - kinematic model (see Appendix) indicate that at bombarding energies $\geq 80 \mathrm{MeV} / \mathrm{u}$ impact paramcters larger than $4 \mathrm{fm}$ can lead to participant - spectator ("fireball" [Bo 73, We 76, Go 77]) types of reactions rather than to incomplete fusion.

In addition, studies of the two above questions can give important information regarding the multifragment decay process which should become important at higher energy. If multifragment decays (defined as 3 or more fragments with $Z>2$ ) are governed 
by a statistical mechanism, then the important parameter is the nuclear temperature. if, however, the mechanism is dynamic, then we expect that the bombarding energy may be the quanity of interest. Recent work has indicated that the excitation energy of the system, rather than the bombarding energy, is more strongly comelated to the average complex fragment multiplicity $[\mathrm{Ha}$ 87, $\operatorname{Tr} 88]$.

The goal of this project is to test whether the incomplete fusion - compound statistical decay model can alsc explain CF emission from asymmetric reactions at larger bombarding energies and correspondingly larger excitation energies. The tery asymmetric ${ }^{139} \mathrm{La}+{ }^{12} \mathrm{C}$ system has been studied at 50,80 and $100 \mathrm{MeV} / \mathrm{u}$, and the more symmetric ${ }^{139} \mathrm{La}+{ }^{27} \mathrm{Al}$, nat $\mathrm{Cu}$ and ${ }^{197} \mathrm{Au}$ systems at $80 \mathrm{MeV} / \mathrm{u}$. Previous work has indir ated the disappearance of the fusion process from evaporation residues [Bo 85c, $\mathrm{Au}$ 86] or coincident fission fragments [Ha 87] studies. However it has since been shown in lighter systems that complex fragment emission can be a more sensitive probe for fusion or incomplete fusion products [P1 89]. Thus these experiments focus on detecting fragments of $Z>2$ emitted inclusively and in coincidence with other $C F$.

The data discussed in this thesis were taken in two separate experiments with two completely different cetection apparatuses. The experimental details will be viscussed serially. For clarity, the $50 \mathrm{MeV} / \mathrm{u}{ }^{139} \mathrm{La}+{ }^{12} \mathrm{C}$ results will be discussed along with the ${ }^{139} \mathrm{La}+{ }^{12} \mathrm{C}$ results at 80 and $100 \mathrm{MeV} / \mathrm{u}$. The data on heavier targets from the $80 \mathrm{MeV} / \mathrm{u}$ experiment will be discussed in a separate section. 


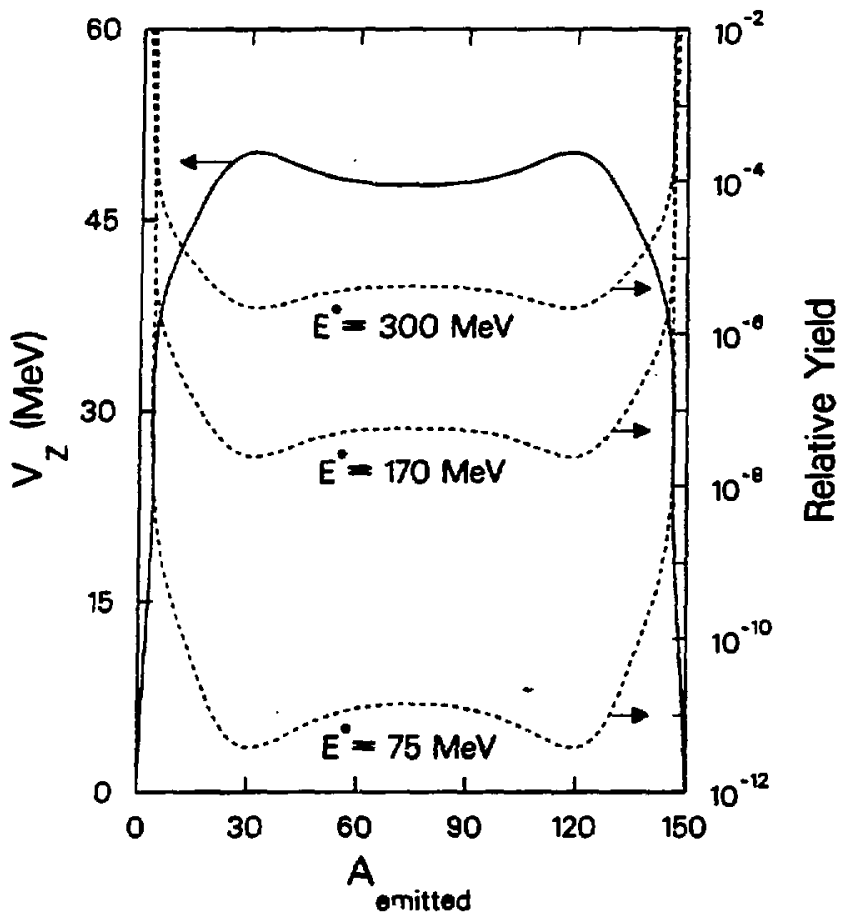

XaL $196-2320$

Figure I.1 Liquid drop conditional barriers $\left(V_{Z}\right)$ at $J=0$ for a ${ }^{139} \mathrm{La}$ compound nucleus as a function of the mass of the emitted fragment (solid line - left scale) [Da 85], and the corresponding relative yields calculated from equation (6) at excitation energies of 75,170 and $300 \mathrm{MeV}$ (dashed line - right scale). 


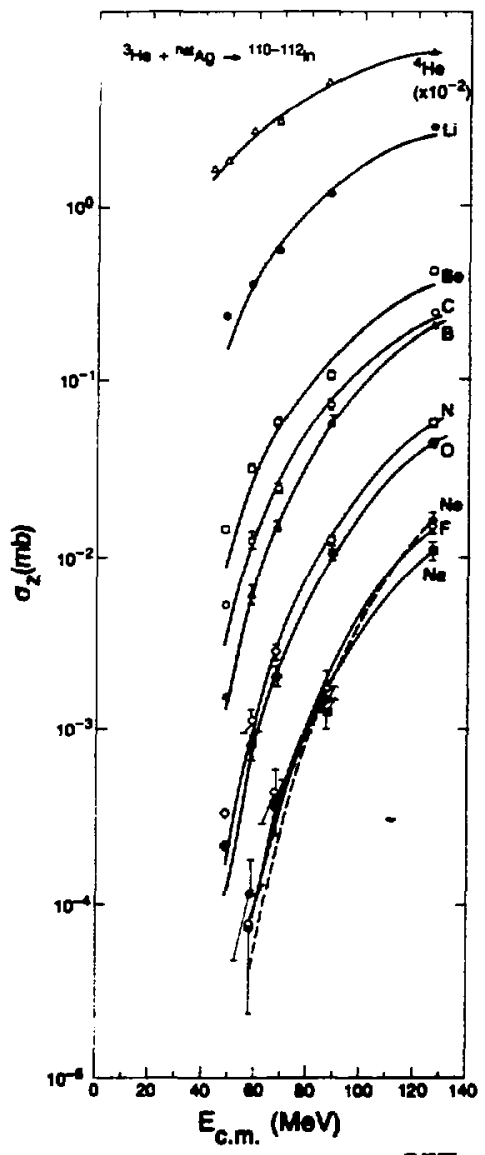

Figure I.2 Excitation functions for complex fragment emission from ${ }^{110-112}$ In compound nuclei. The symbols with error bars are the experimental points. The lines are statistical model calculations of first chance complex fragment emission [Mc 85]. 


\section{EXPERIMENTAL}

\section{A. Choice of Projectile and Target}

The total mass of the projectile and target is constrained by the mass of the system that one wishes to study. Systems of the desired mass can, however, be formed with a variety of entrance channel mass asymmetries. The of use very asymmetric entrance channels, in which a broad range of impact parameters leads to complete overlap of the projectile and target, and possibly fusion, should simplify, or at least limit the number of competing processes as compared with symmetric reactions, where each impact parameter is associated with a different overlap, and perhaps a different mass transfer. Previous studies have shown that the use of very asymmetric entrance channels gives rise to sources with well-defined velocities, masses and excitation energies [Ch $86, \mathrm{Ch} 88 \mathrm{a}, \mathrm{Ch} 88 \mathrm{~b}, \mathrm{Ha}$ 89 , Pl 89, Ch 89]. We have thus chosen to study a systems of mass $150-200$ using the asymmetric ${ }^{139} \mathrm{La}+{ }^{12} \mathrm{C},{ }^{27} \mathrm{Al}$, and ${ }^{\text {nat }} \mathrm{Cu}$ entrance channels.

The ${ }^{139} \mathrm{La}$-induced experiments described in this thesis take advantage of the unique capabilities of the Lawrence Berkeley Laboratory BEVALAC by using reverse (or inverse) kinematics, that is an asymmetric projectile - target combination with the heavier nucleus used as the projectile. The technical details of this procedure are quite different from those of a normal kinematics experiment, in which the lighter reaction partner is used as the projectile. Additional information can be obtained using reverse kinematics, although the physics of the reaction is, of course, identical in both cases.

Figure II.1 schematically illustrates the kinematics of a compound binary decay process in a reaction induced with reverse kinematics. The interaction between the target and the much heavier projectile creates a source moving with a large laboratory velocity denoted by $V_{\text {source. }}$ The binary decay partners are emitted at all angles in the source frame

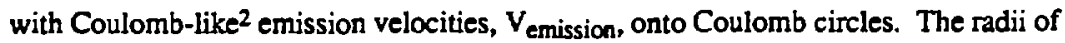
these circles are dependent upon the Coulomb energy in the decay process and the mass 
asymmetry of the decay partners, with the lighter fragments having larger emission velocities due to momentum conservation.

Typically in reverse kinematics the source velocity is larger than the emission velocity, so the laboratory velocity distributions show two peaks at a given laboratory angle. The velocity vector $\mathrm{V}_{\mathrm{a}}$ corresponds to forward emission in the source frame, and $V_{b}$ corresponds to backward emission with the same emission velocity. For each physical binary event there is a second fragment emitted $180^{\circ}$ in the source frame from the first fragment and located on another Coulomb circle. The respective radii of the circles depend upon the Coulomb energy and mass asymmetry as discussed above.

The large velocity of the source makes it rather easy to detect and identify the heavier fragments, which have very small velocities in the source frame and which are virtually impossible to identify with normal kinematics. Recent experiments using normal kinematics have shown that, even with $4 \pi$ detector systems, a large fraction of the mass can exist as large fragments that cannot be detected oridentified [So 89]. With reverse kinematics these fragments are easy to detect and identify. The large source velocity also gives rise to a forward focusing of the reaction products, so modest sized detectors can have very large effective solid angles. This effect is particularly important in detecting coincident fragments.

Along with the advantages of reverse kinematics relative to normal kinematics there are disadvantages as well. In contrast to normal kinematics, where the experimentally measured quantities are not much different from the relevant (center-of-mass) quantities, in reverse kinematics quantities are extracted by taking the difference between two large numbers. For instance, the difference between the source velocity and the beam velocity may be a small percentage of the original beam velocity. This may attach some uncertainty to such quantities as momentum transfer and excitation energy. Additionally, the advantage of the much larger geometric efficiency turns into a disadvantage when the multiplicities per event become too large, requiring a highly segmented detector. Obviously this is a special 
problem with protons and alpha particles where the multiplicity per event can be $\geq 10$

B. $50 \mathrm{MeV} / \mathrm{u}^{139} \mathrm{La}{ }^{12} \mathrm{C}$

1. Beam, Targets, and Detection Hardware

The $50 \mathrm{MeV} / \mathrm{u}{ }^{139} \mathrm{La}$ beam was provided by the Lawrence Berkeley Laboratory BEVALAC Accelerator Complex. The 13\%La ions were produced by the Abel injector at the SuperHILAC, accelerated to $1.2 \mathrm{MeV} / \mathrm{u}$ and stripped to a charge state of +31 , and further accelerated to $5 \mathrm{MeV} / \mathrm{u}$ before injection into the Bevatron. In the Bevatron the ions were accelerated to their final energy of $50 \mathrm{MeV} / \mathrm{u}$ and extracted at a magnetic field of 3035 Gauss. The extracted beam was delivered into the $60^{\prime \prime}$ diameter scattering chamber in the beam 44 cave in the Bevatron EPB Hall.

The beam energy at the BEVALAC is determined from the radial position of the beam at extraction and from the synchrotron field, and is estimated to be known to $\pm 1 \%$. The beam spot was approximately circular and about $1.5 \mathrm{~cm}$ in diameter, with an estimated beam divergence of $1^{\circ}$. The beam quality was very poor during this experiment. Targetout runs show the presence of both heavy fragment background, due to the scattering of primary beam particles, and light particle background, from reaction products generated in the beam pipe and collimators.

Self-supporting targets of ${ }^{12} \mathrm{C}$ and ${ }^{197} \mathrm{Au}$ (for elastic scattering calibrations) with thicknesses of 2.2 and $4.0 \mathrm{mg} / \mathrm{cm}^{2}$, respectively, were prepared by evaporation at the Lawrence Berkeley Laboratory target-making facility. The target thicknesses were chosen such that the bram lost no more than $1 \%$ of its energy within the targets.

The experimental set-up is illustrated in Figure II.1. Two circular detector telescopes of $2^{\prime \prime}$ diameter were centered about the beam at 5.5 degrees, and covered from $3^{\circ}$ to $8^{\circ}$ in-plane, and $-2.5^{\circ}$ to $2.5^{\circ}$ out-of-plane. The detectors were placed $58.7 \mathrm{~cm}$ from the target, and each subtended a solid angle of $5.9 \mathrm{msr}$. Although this solid angle is very small in the lab, the focusing of reaction products due to the reverse kinematics allows for reasonable detection efficiencies for both inclusive and coincidence events. The 
experimentally determined singles efficiency for fragments of $Z=25$ was approximately $10 \%$. For symmetric and nearly symmetric decays, the efficiency for detecting both fragments in coincidence was approximately $3 \%$. The coincidence efficiency was greater than $1 \%(10 \% \times 10 \%)$ because of the correlation between the coincident fragments.

A schematic of one of the four-element GaSP telescopes (Gas-Silicon-siliconPlastic) is shown in Figure II.2. Each GaSP has a gas ionization detector as a $\Delta E$ element, followed by a $2 \mathrm{~mm}$ thick $\mathrm{Si}(\mathrm{Li})$ solid state detector, a second $1.5 \mathrm{~mm}$ thick $\mathrm{Si}(\mathrm{Li})$, and finally a 2" or 3" thick plastic scintillator. The Frisch-gridded transverse-field ionization chambers of $14 \mathrm{~cm}$ length were pressurized with 200 torr of P-10 (90\% Ar and $10 \%$ $\mathrm{CH}_{4}$ ) gas mixture. Each chamber had a two segment anode, but only the signal from the second (longer) anode was recorded. Operating voltages of $+400 \mathrm{~V}$ for the anodes and $800 \mathrm{~V}$ for the cathode were used in each ion chamber. The Frisch grid was held at ground potential. The 3" diameter $\mathrm{Si}(\mathrm{Li})$ detectors were fabricated at the Lawrenc: Berkeley Laboratory Silicon Detector Laboratory, and were operated with a bias voltage of $+300 \mathrm{~V}$. The plastic scintillators were Bicron corporation model \#BC-400, and were optically coupled to a type HTV R2060 photomultiplier tube provided with $+700 \mathrm{~V}$. Figure III. 3 is a photograph of the two telescopes. In this figure, the ion chamber was removed from the further telescope to show a $\mathrm{Si}(\mathrm{Li})$ detector.

The detector telescopes were position-sensitive both in- and out-of-plane. The back of the $\mathrm{Si}(\mathrm{Li})$ detectors, on which the +300 Volt bias was applied, had a Au ohmic contact from which electrons were cullected to determine the energy loss of the fragments. A resistive Pd layer ran across the front face of the detectors. The in-plane position was determined by resistive charge division across this layer. At one side of the front face (farther from the beam) the resistive layer was grounded through 50 ohms. On the other side the holes were collected. The in-plane position was determined by dividing the signal from the holes by the total energy signal from the electrons. Vertical strips of Au across the front face of the detector produced a more uniform resistive gradient and partially 
compensated for the circular geometry of the detectors. Thus the position signal mainly depended upon the horizontal position of the fragment, rather than upon the total distance to the contact, as it would with no Au strips.

The out-of-plane position signals were measured from the electron drift time in the gas $\Delta E$ section. A particle striking the first $\mathrm{Si}(\mathrm{Li})$ detector generated a prompt timing signal which was used to start a drift time time-to-amplitude converter (TAC). The anode signal of the ionization chamber was used as the stop signal for the TAC. This signal was generated when the electron cloud, which was produced when a charged particle traversed the ionization chamber, passed through the Frisch grid. The time difference measured in the TAC was a measure of the time necessary for the electron cloud to reach the Frisch grid, and depended upon the vertical position of the fragment. The gas detectrs were operated with an electric field per unit pressure of $\sim 0.6 \frac{\mathrm{V}}{\mathrm{cm} \text {-torr }}$ which should correspond to drift velocities of about $12 \mathrm{~cm} / \mu \mathrm{sec}$ [Ch 79]. However, the measured drift times were somewhat greater than predicted by this value.

The beam current was monitored using a Faraday cup and a current integrator. The beam stop in the Faraday cup was kept at a potential of $+200 \mathrm{~V}$ to limit the scattering of electrons from the stop, which would give each beam particle a larger effective charge. The current integrator electronics were calibrated by delivering a known current into the preamplifier. Since the Faraday cup itself could not be calibrated with elastic scattering, the uncertainties in the absolute beam intensity are estimated to be $\sim 50 \%$.

\section{Electronics and Logic}

The electronic scheme for this experiment is shown in Figure II.4. The master gate for the electroniss was a valid coincidence between the $\Delta E$ (second anode of gas section) and the $\mathrm{E}$ (electron signal) of the first $\mathrm{Si}(\mathrm{Li})$ detector. Amplitide thresholds were set on the prompt signals coming from the timing amplifiers to allow $\mathrm{Li}$ and heavier fragments to trigger the electronics, but to exclude He and lighter particles. Of course, it is not possible 
to achieve a complete separation, and there were some He that did trigger, and some $\mathrm{Li}$, and perhaps even heavier ions, that did not trigger the electronics.

Inclusive and coincidence events were written event-by-event onto magnetic tape using a PDP 11/45 with a Multi-Branch Driver reading standard CAMAC modules (Ortec AD811 ADCs). The data were analyzed off line using a VAX 780 computer and the data analysis package LISA [ $\mathrm{Li} 88]$ with user-written subroutines.

\section{Calibrations and Analysis}

The energy response of the gas ionization chamber anodes and the $\mathrm{Si}(\mathrm{Li})$ detectors was calibrated using elastically scattered $50 \mathrm{MeV} / \mathrm{u}^{139} \mathrm{La}$ ions (from the ${ }^{197} \mathrm{Au}$ target), and the zero-channel of the slectronics as determined from pulser calibrations. Corrections for the energy losses [Hu 78] in the targets, the $4.7 \mathrm{mg} / \mathrm{cm}^{2} \mathrm{naPb}$ foils (used in front of each detector for electron suppression), and the $175 \mu \mathrm{g} / \mathrm{cm}^{2}$ mylar gas windows, along with a correction for the pulse height deficit in the Si(Li) detectors [Mo 78] were performed for the calibration beam prior to the calibration procedure, and for each detected fragment.

The energy calibration was estimated to be accurate to approximately $2 \%$, but systematic errors in the pulse height defect (PHD) parametrization could make the absolute error larger. The PHD correction is not considered to be very accurate for ${ }^{139} \mathrm{La}$ ions at these energies. With one exception, which is discussed below, systematic errors should affect both the calibrations and the data similarly, and thus will not greatly affect any of the extracted quantities.

The absolute position calibrations were done using a position mask of $2 \mathrm{~mm}$ diameter holes equally spaced $(5 \mathrm{~mm})$ in- and out-of-plane. The raw Energy (electron signal) vs. Position $x$ Energy (hole signal) and drift time spectra of the detectors are shown in Figure II.5. The theoretical resolution was approximately $2 \mathrm{~mm}$ or 0.2 degrees in the laboratory. However, the actual uncertainty in the measured angles was about $0.8^{\circ}$ due to the beam spot size (mainly) and the beam divergence.

Slight $(\sim 10 \%)$ quadratic dependences of the anode energy signal upon the vertical 
position of the detected fragment, and of the drift time upon the anode signal were found. Corrections were performed using elastic scattering calibration data taken with the position masks in front of the detectors. The corrected and calibrated position spectra are shown in Figure II.6.

The $\mathrm{Z}$ identification of the detected fragments was performed by first converting the $\Delta E$ and $E$ channels into energy units (MeV) as described above. Plots of $E$ versus $\Delta E$ (or vice versa) are typically used to extract the atomic charge of the detected fragments. The standard method is to draw tree form gates around each of the "Z-lines" in the E - $\Delta E$ maps (see Figure II.7). However, using this method to extrapolate the particle identification beyond the region in which the lines are visible is not very accurate. A better method is to make use of the classical Bohr equation [Bo 48] for the energy loss of a charged particle in a medium:

$$
-\frac{d E}{d x}=\frac{4 \pi e^{4} Z^{2} \rho z}{m_{e} v^{2}} \ln \left[\frac{m_{e} v^{3}}{2 Z e^{2} \omega}\right] .
$$

Here $e$ is the electron charge, $m_{e}$ is the mass of the electron, $\rho, z$, and $\omega$ are respectively the number density of atoms, the atomic number, and the classical frequency of motion of the elecison in the stopping material; and $\mathrm{Z}$ and $\mathrm{v}$ are the effective charge and velocity of the charged particle. Neglecting the slowly varying logarithmic term we have:

$$
-\frac{d E}{d x} \propto \frac{Z^{2}}{v^{2}} \propto \frac{M Z^{2}}{E} \propto \frac{Z^{3}}{E}
$$

where the additional assumption that $\mathrm{Z} \propto \mathrm{M}$ has been made. By equating $\mathrm{dE} / \mathrm{dx}$ with the energy loss in the $\Delta E$ detector (assumption of a thin detector), we have the equation of a hyperbola for each separate $Z$-value, $\Delta E \cdot E=a(Z)$.

In practice, the equation $\left(\Delta E+a_{1}\right)\left(E+a_{2}\right)=a_{3}$ was used to fit the $E$ versus $\Delta E$ 
curves for the known $Z$-lines of $Z \leq 25$ and $Z=57$ (from elastic scattering). Then the series of fitting parameters $a_{1}, a_{2}$, and $a_{3}$ were themselves fit as functions of $Z$, as quadratic, linear, and cubic functions respectively. Finally, to extract the charge of each detected fragment, the cubic equation in $Z$ was soived for each $\Delta E-E$ pair. It was necessary to use two different particle identification functions for $Z<8$ and for $Z \geq 8$, and to rescale the particle identification values to units of atomic number. Figure II.8(a) shows the extracted atomic numbers for one of the detector telescopes. Peaks corresponding to atomic numbers up to 26 are visible.

The average mass associated with each Z-value must be determined in order to calculate fragment velocities from the measured energies and Z-values. This is not a straightforward determination, since the primary fragments are excited and sequentially evaporate light particles. The evaporation code PACE has been used to estimate the secondary masses following light particle evaporation [Ch $88 \mathrm{a}$ ]. The average mass for each Z-value determined from the PACE simulations is approximately A $=2.08 \mathrm{Z}+$ $0.0029 Z^{2}$, which is in agreement with experimentally determined average masses [Au 87]. These simulations show that when the excitation energies per fragment are $\geq 1 \mathrm{MeV} / \mathrm{u}$ the secondary mass for a given charge is independent of the initial excitation energy, and corresponds to a mass slightly more proton-rich than the valley of beta-stability. In the simulations, the fragments first emit neutrons until they reach the above curve, and then they emit both protons and neutrons to follow the curve down to smaller masses.

In actuality, the mass of each fragment was calculated using the equation $A=2.17 \mathrm{Z}$ $+0.0027 Z^{2}$. This mass parametrization, which predates the more accurate one given above, predicts masses for each $Z$-value that are about $4 \%$ larger than those from the mass parametrization based on the PACE simulations. This corresponds to approximately $2 \%$ smaller velocities. Quantities such as emission velocities and source frame angular distributions depend very little on the laboratory velocity of the fragments because systematic errors in the lab velocities and in the source velocity cancel, but the extracted 
source velocity and momentum transfer do depend upon the parametrization.

Recently acquired data show that the empirical Moulton formula [Mo 78] greatly underestimates pulse height deficits for ions of $Z>40$. The slope of the energy calibration used for the data analysis is based on elastically scattered ${ }^{139} \mathrm{La}$ ions, and is estimated to be about $6 \%$ larger than the "true" slope calculated with a more realistic determination of the pulse height deficit. The systematic error in the mass parametrization should be nearly compensated by the underestimation of the pulse height deficit of the elastically scattered ${ }^{139} \mathrm{La}$ ions. Thus to within about $1-2 \%$ (in velocity) the systematic errors from the pulse height deficit and the mass parametrization should cancel.

The raw data were calibrated and written to "sorted" files with parameters of $Z_{1}$, $Z_{2}, E_{1}, E_{2}, v_{1}, v_{2}, X_{1}, X_{2}, Y_{1}$, and $Y_{2}$, which were then used for further analysis. The time difference between the first $\mathrm{Si}(\mathrm{Li})$ detectors of both telescopes was used to eliminate random events from the coincidence data. It is estimated that no more than a $1 \%$ contamination of random events makes it through the time gate.

C. 80 and $100 \mathrm{MeV} / \mathrm{u}{ }^{139} \mathrm{La}+{ }^{12} \mathrm{C}_{\mathrm{C}^{27}}{ }^{\mathrm{Al}}{ }^{\text {nat }} \mathrm{Cu}$, and ${ }^{197} \mathrm{Au}$

1. Beam, Targets, and Detection Hardware

The ${ }^{139} \mathrm{La}$ ions were produced in the Abel injector of the SuperHILAC as during the $50 \mathrm{MeV} / \mathrm{u}$ experiment, but further accelerated to $8.5 \mathrm{MeV} / \mathrm{u}$ and stripped to a charge state of +48 before injection into the Bevatron for the final accelerations to 80 and 100 $\mathrm{MeV} / \mathrm{u}$ at main field settings of 2500 and 2800 Gauss. The beam was again delivered into the $60^{\prime \prime}$ scattering chamber in Beam 44. The beam spot for this experiment was oval, about 2-3 $\mathrm{mm}$ in width and $1 \mathrm{~cm}$ in height. The estimated beam divergence was $1^{\circ}$.

The targets of ${ }^{12} \mathrm{C},{ }^{27} \mathrm{Al}$, ${ }^{\mathrm{na}} \mathrm{Cu}$, and ${ }^{197} \mathrm{Au}$ had thicknesses of 3.7, 5.5, 4.0, and $4.0 \mathrm{mg} / \mathrm{cm}^{2}$ respectively, and were prepared as above. The target thicknesses were again chosen such that no more than $1 \%$ of the beam energy was lost in the target. Thus at these higher energies it was possible to have slightly thicker targets

A new local coliimation system was introduced into the scattering chamber prior to 
these experiments. The collimator system consisted of two parts: a 3" thick circular Al plate that fit into the port between the scattering chamber and the beam line, and two circular rings of 1/8" thick Ta: an upstream ring of 3/8" diameter and a "clean-up" ring of $1 / 2$ " diameter. The Al plate was necessary to eliminate secondary beams of ${ }^{139} \mathrm{La}$ stripped to higher charge states in the external beam line, and bent to larger angles in the bending magnet just upstream of the scattering chamber. These secondary beams produced a large background at angles $\leq 15^{*}$. The new collimator system significantly improved the quality of the beam, virtually eliminating the background scattering of heavy beam-like particles into the detectors that was seen at the lower energy (compare Figures II.7 and II.9).

A schematic of one of the detector telescopes employed in this experiment is shown in Figure II.10. Each detector telescope had 4 elements - a $300 \mu \mathrm{m}$ thick Si detector used as a $\Delta E$ element, followed by two $5 \mathrm{~mm}$ thick $\mathrm{Si}(\mathrm{Li})$ detectors, and finally a $7.5 \mathrm{~cm}$ thick plastic scintillator. The detector telescopes were $5.5 \mathrm{~cm} \times 5.7 \mathrm{~cm}$, and each telescope had an active area of $4.5 \mathrm{~cm} \times 4.5 \mathrm{~cm}$. The large dead regions were due to construction requirements of the $5 \mathrm{~mm} \mathrm{Si}(\mathrm{Li})$ devices. A photo of a detector telescope is shown in Figure II.11.

Eleven such detector telescopes were arranged into a $3 \times 4$ array about the beam. Figure II.12 is a beam's eye photo of the array showing the close packed geometry and the missing element through which the beam passes. Each element of the array was positioned $91 \mathrm{~cm}$ from the target, and had a solid angle of $2.4 \mathrm{msr}$. The total array covered from approximately $-5^{*}$ to $+9^{\circ}$ in-plane and from $-5^{\circ}$ to $+5^{\circ}$ out-of-plane, with a $\pm 2.2^{\circ}$ gap in- and out-of-plane about $0^{\circ}$. There were several advantages of this detection system over the older system. The most obvious was the number of telescopes in the apparatus. Other advantages will be discussed below.

The $300 \mu \mathrm{m}$ and $5 \mathrm{~mm} \mathrm{Si}(\mathrm{Li})$ devices were fabricated at the Lawrence Berkeley Laboratory Silicon Detector Laboratory. The $300 \mu \mathrm{m}$ detectors were biased with voltages

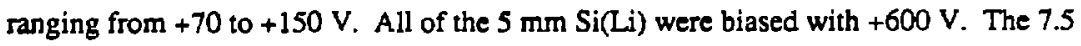


$\mathrm{cm}$ plastic scintillators were purchased from Bicron, model \#BC-400. The photomultiplier tubes (type HTV R2060) behind each scintillator were operated with +600 to $+800 \mathrm{~V}$.

The position determination was done in a slightly different manner than described above. Each $\mathrm{Si}$ or $\mathrm{Si}(\mathrm{Li})$ detector was position sensitive in one dimension. The electrons were collected at a Au ohmic contact on the back of the detectors, and the holes were collected on the front. The front of each device was divided into 15 Au strips of $2.4 \mathrm{~mm}$ width, separated by 14 resistive strips of $0.6 \mathrm{~mm}$ width. As before, one side of the front face of the detectors was terminated to ground through $\mathbf{5 0}$ ohms, so that the position of the fragments was again proportional to the hole signal divided by the electron signal. However, with these devices all of the resistance appears between the Au strips so that the position signal is discrete. The discrete nature of the devices makes them self-calibrating. No position mask for absolute calibration was necessary. The square geometry of the devices also eliminated much of the non-linear edge effects associated with the circular detectors described above. The strips in each detector were arranged orthogonally to each other to give both in- and out-of-plane position determinations, with a redundant in-plane measurement from the second set $5 \mathrm{~mm} \mathrm{Si(Li)} \mathrm{detectors.}$

A gas ionization chamber followed by a plastic scintillator was used to monitor the beam current. A Ta mask with an array of holes in it was placed directly in front of the scintillator during the experiment to decrease the scintillator counting rate to measurable levels. Following the experiment, the ion chamber current was calibrated versus the absolute beam intensity uring the scintillator, with mask, at high intensity; and the scintillator, without mask, at low intensity. The uncertainty in the absolute beam monitoring was estimated to be $\sim 20 \%$.

2. Electronics and Logic

The electronics scheme for this experiment was very similar to that described above for the $50 \mathrm{MeV} / \mathrm{u}$ experiment. A master gate was gemerated by a coincidence between the $300 \mu \mathrm{m} \mathrm{Si} \mathrm{detector} \mathrm{and} \mathrm{the} \mathrm{first} 5 \mathrm{~mm} \mathrm{Si}(\mathrm{Li}$ ) of any of the 11 array elements (see Fig 
II.13(a) and (b)). As befort, the logic discriminators were set to exclude protons and alpha particles from triggering the acquisition system, but to allow heavier fragments. Wher a valid $\Delta E$ - E1 coincidence was generated, 44 energy signals ( $\triangle E, E 1, E 2, P 1$ for 11 telescopes), 33 position signals ( $\mathrm{X} \triangle \mathrm{E}, \mathrm{YE} 1$, and $\mathrm{XE} 2$ for each telescope) and a timing signal for each telescope were digitized, read out by a VME based processor, and transferred over Ethemet to a VAX 780 for transfer to magnetic tape and on-line monitoring. The data anaiysis program LISA [Li 88], with user-writter، subroutines, was used for on-line monitoring (in conjunction with a second program for dispiay of twodimensional spectra) and off-line data analysis on both a VAX 780 and a VAXstation 2000.

\section{Calibrations and Analysis}

The energy calibrations were performed by determining the zero-channel of all of the ADCs, and by stopping $80 \mathrm{MeV} / \mathrm{u}:{ }^{39} \mathrm{La}$ and ${ }^{40} \mathrm{Ca}$, and $100 \mathrm{MeV} / \mathrm{u}{ }^{139} \mathrm{La}$ calibration beams directly in the first $5 \mathrm{~mm} \mathrm{Si}(\mathrm{Li}$ ) detector (both with and without the $300 \mu \mathrm{m} \mathrm{Si}$ in front). This was accomplished by removing the local collimator and by defocusing the beam with the quadrupole magnets directly in front of the scattering chamber. The second set of $5 \mathrm{~mm} \mathrm{Si}(\mathrm{Li})$ detectors was calibrated in the same manner with $80 \mathrm{MeV} / \mathrm{u}{ }^{40} \mathrm{Ca}$ beams and the zero-channel of the ADCs. The Plastic detectors were calibrated with $80 \mathrm{MeV} / \mathrm{u}$ ${ }^{20} \mathrm{Ne}$ and ${ }^{4} \mathrm{He}$ beams. Corrections for the energy losses in the target and in the $1.5 \mathrm{mg} / \mathrm{cm}^{2}$ Au foils 'used for electron suppression were applied. These corrections were very small for all of the measured fragments.

The $80 \mathrm{MeV} / \mathrm{v},{ }^{139} \mathrm{La}$ and ${ }^{40} \mathrm{Ca}$, and the $100 \mathrm{MeV} / \mathrm{u}{ }^{139} \mathrm{La}$ calibration points indicate that the parametrization of Moulton et al. [Mo 78] grossly underpredicts the pulse height deficit (PHD) for ${ }^{139} \mathrm{La}$ ions at these energies. A new correction for the PHD was developed based upon data taken at the $88^{\prime \prime}$ Cyclotron and the 80 and $100 \mathrm{MeV} / \mathrm{u}^{139} \mathrm{La}$ calibration points.

Ions of $\mathrm{Fe}, \mathrm{Zn}, \mathrm{Kr}$, and $\mathrm{Xe}$ with energies of $8.75 \mathrm{MeV} / \mathrm{u}$ from the 88" Cyclotron vere stopped directly in a $5 \mathrm{~mm} \mathrm{Si}(\mathrm{Li})$ detector similar to those used in this experiment. To 
better than $1 \%$, the ratio of the PHD to the measured pulse height $(\mathrm{PH})$ was found to be a pure cubic function of the fragment charge, PHD $=\left(7.87 \times 10^{-7} \mathrm{Z}^{3}\right)$ PH. Using the PHD calculated at $8.75 \mathrm{MeV} / \mathrm{u}$ for ${ }^{139} \mathrm{La}$ ions, and those measured directly at 80 and $100 \mathrm{MeV} / \mathrm{u}$, the following correction for the PHD as a function of energy was obtained:

$$
E=E^{\prime} Z^{3}\left[7.87 \times 10^{-7}-C\left(\frac{E^{\prime}}{A}-8.75\right)\right]
$$

Here $E$ is the "true" energy, $E$ ' is the measured energy from the linear energy calibration, $A$ is the fragment mass and $\mathrm{C}$ is a constant calculated separately for each of the $\mathrm{Si}(\mathrm{Li})$ detectors. Since the PHD is thought to be primarily an end-of-range effect, the above correction was only applied for detectors in which the fragments stopped. Thus no PHD corrections were applied to the energies measured in the $300 \mu \mathrm{m} \mathrm{Si} \mathrm{detectors.} \mathrm{This}$ empirical parametrization should predict the absolute PHD to approximately $10 \%$, corresponding to less than a $1 \%$ uncertainty in the total energy.

Raw spectra of $\triangle E$ vs $X \triangle E$ and $E$ vs $Y E$ are shown in Figure II.14. Lines corresponding to the 15 discrete Au strips are clearly visible. The position spectra show small energy dependences associated with the very small energy channels. Kaufman et al [Ka 70] associate these non-linearities with DC offsets in the shaping amplifiers and the ADCs, and propose a simple method to eliminate the dependences. In the present analysis a different method was used. The position lines corresponding to each strip were plotted versus energy and then corrected to their asymptotic values at large energy using a twodimensional least squares fit (deviation versus position and energy). This method was not found to improve upon the simpler method of Kaufman ct al.

The theoretical position resolution of the new detectors is $\pm 1.5 \mathrm{~mm}$ or 0.1 degree, better than what was achieved with the conunuous detectors. Figure II.15 shows the corrected and calibrated position spectra for the $\Delta E$ and $E 1$ detectors. The overall position resolution of the scattered fragments is again determined mainly by the beam spot size and 
is estimated to be $\sim 0.5^{\circ}$.

The $Z$ identification was performed with a streamlined version of the method reported above. From equation (15) above we see that the product $\Delta \mathrm{E} \cdot \mathrm{E}$ is proportional to $\mathrm{Z}^{3}$, if we assume $\mathrm{Z}$ to be proportional to the mass. Taking the cube root of $\Delta \mathrm{E} \cdot \mathrm{E}$ should give a quantity which is nearly proportional to the atomic number of each fragment. By scaling this quantity linearly with the $\Delta \mathrm{E}$ signal it is possible to achieve excellent resolution. Figure $\Pi .8(\mathrm{~b})$ shows the resulting $\mathrm{Z}$ resulution using this particle identification scheme. Atomic numbers past $Z=50$ are distinguishable in this logarithnic plot. The much improved charge resolution in the 80 and $100 \mathrm{MeV} / \mathrm{u}$ experiment relative to the $50 \mathrm{MeV} / \mathrm{u}$ experiment is due to the larger energy loss in the thicker Si $\Delta \mathrm{E}$ elements, and to the better energy resolution of the solid state detectors as compared with the gas ionization detectors.

The mass parametrization used in this experiment was $\mathrm{A}=2.08 \mathrm{Z}+0.0029 \mathrm{Z}^{2}$ which was determined from PACE [Ga 80] simulations by Charity et al. [Ch 88a]. As described above, this parametrization is in agreement with experimentally determined average masses [Au 87] in this bombarding energy regime.

As before, the calibrated data were written to sorted files. Inclusive files were written with parameters of $Z, E, \theta, \varphi$, a time signal (TDC), and the detector identification. Coincidence files were also written that included a multiplicity parameter and each of the above parameters for every coincident fragment. A gate on the TDC spectrum of each detector was set to eliminate random events. It is again estimated that no more than $1 \%$ of the coincidence data is due to random events getting through the TDC gates. 


\section{Reverse Kinematic Reactions \\ Experimental Set-up for $50 \mathrm{MeV} / \mathrm{u} \mathrm{La}+\mathrm{C}$}

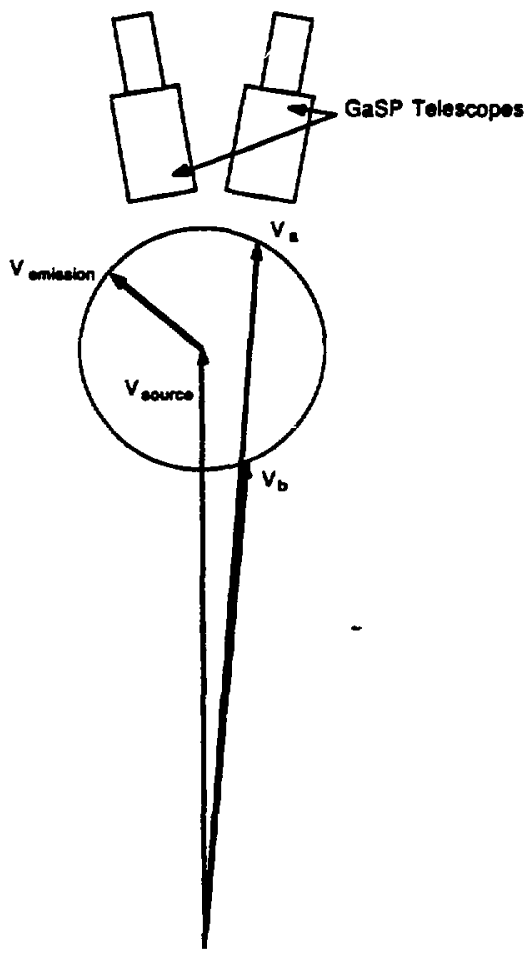

$x$ x $196 \cdot 2321$

Figure II.1 Schematic loci of events from compound binary decay in a reverse kinematics reaction. A source with a sharply defined velocity $\left(V_{\text {source }}\right)$ emits fragments with velocity ( $V_{\text {emission}}$ ) onto an isotropic Coulomb ring. The vectors $V_{a}$ and $V_{b}$ show the two laboratory velocities possible at a given laboratory angle, one corresponding to emission forward in the source frame $\left(V_{a}\right)$, and the other to emission backward $\left(V_{b}\right)$. The experimental set-up (GaSP Telescopes) in the $50 \mathrm{MeV} / \mathrm{u}{ }^{139} \mathrm{La}+{ }^{12} \mathrm{C}$ reaction is shown. The two GaSP telescopes span from $3^{\circ}-8^{\circ}$ in the laboratory. 


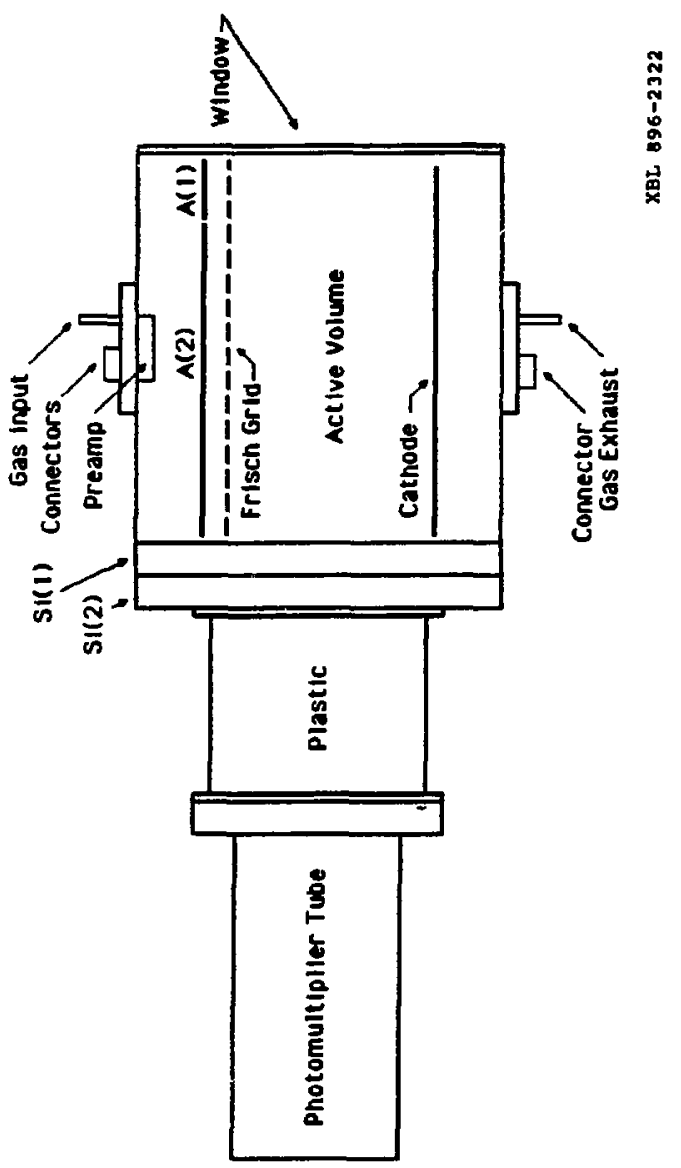

Figure II.2 Schematic drawing of a GaSP (Gas - Silicon - silicon - Plastic) telescope. 


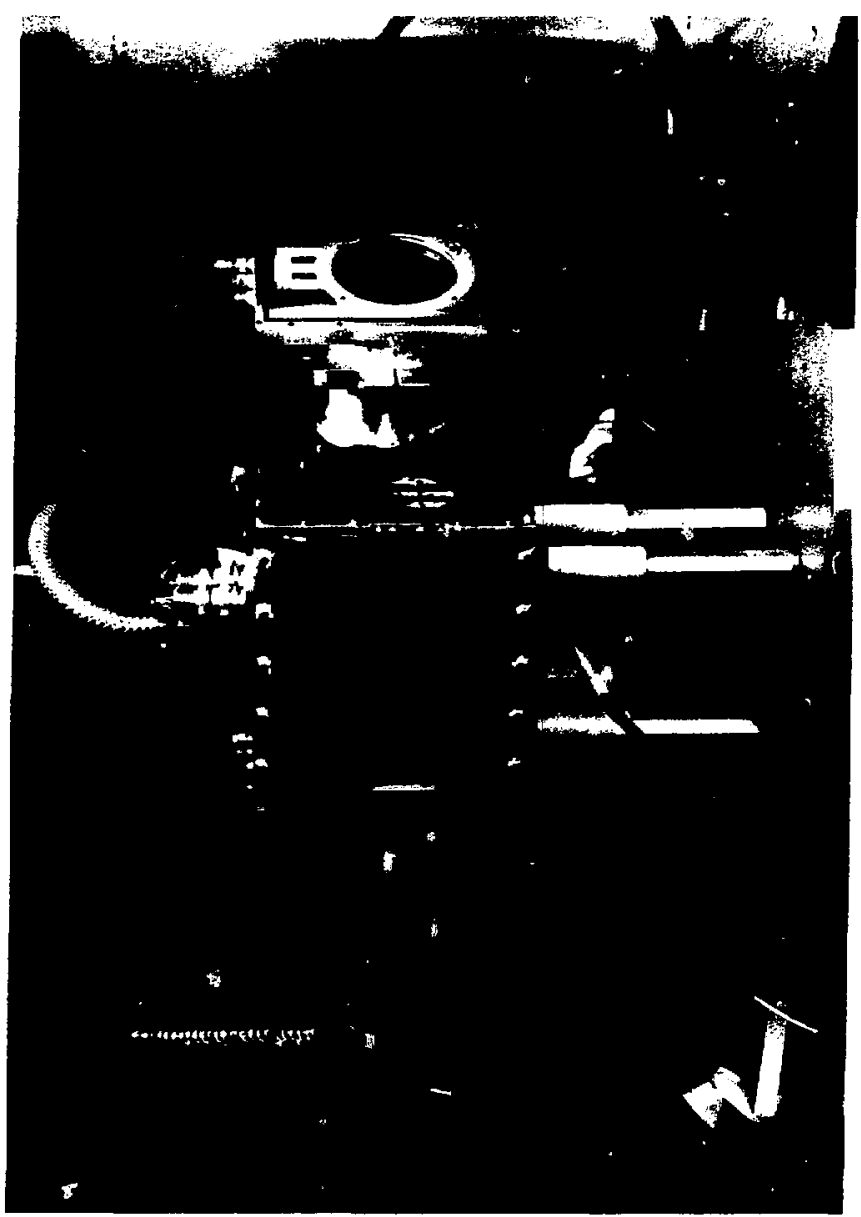

Figure II.3 Photograph of a GaSP telescope (foreground). The ionization chamber window is behind the circular opening. In vacuum, the horizontal and vertical bars support the window. Magnets above and below the opening are for electron suppression. In the background, a $\mathrm{Si}(\mathrm{Li})$ detector similar to those used in the GaSP telescopes is mounted in front of a plastic scintillator. 
Telescope Logic
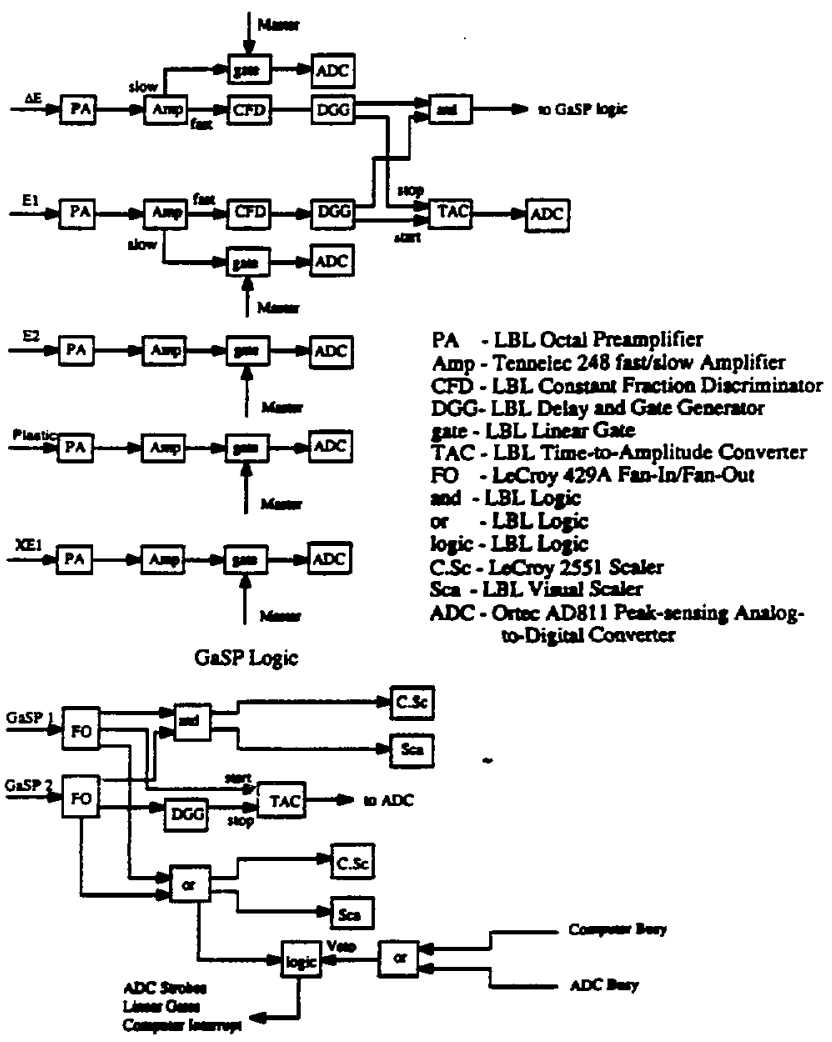

XDL 896-2323

Figure U.4 Electronics diagram for the $50 \mathrm{MeV} / \mathrm{u}{ }^{139} \mathrm{La}+{ }^{12} \mathrm{C}$ experiment. 


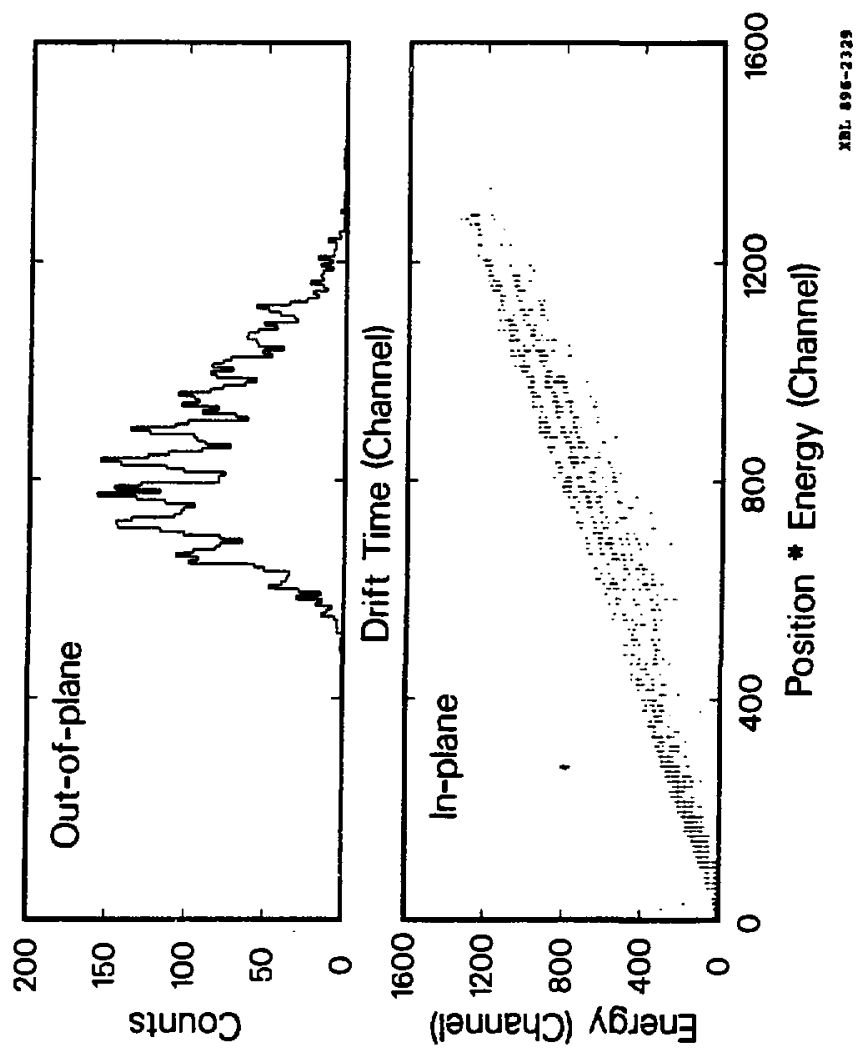

Figure Il.5 Raw position spectra for the $50 \mathrm{MeV} / \mathrm{u}{ }^{139} \mathrm{La}+{ }^{12} \mathrm{C}$ experiment. The top portion of this Figure shows the time difference between the $\mathrm{Si}(\mathrm{Li})$ detector and the gas ionization chamber, which is a measure of the Y-position of the fragment. The bottom portion of the Figure shows the Energy signal from the $\mathrm{Si}(\mathrm{Li})$ detector (electrons) versus the (X-Position x Energy signal) (holes). The peaks in the top spectrum correspond to the vertical columns of the calibration mask and the diagonal lines in the bottom spectrum correspond to the horizontal rows. 


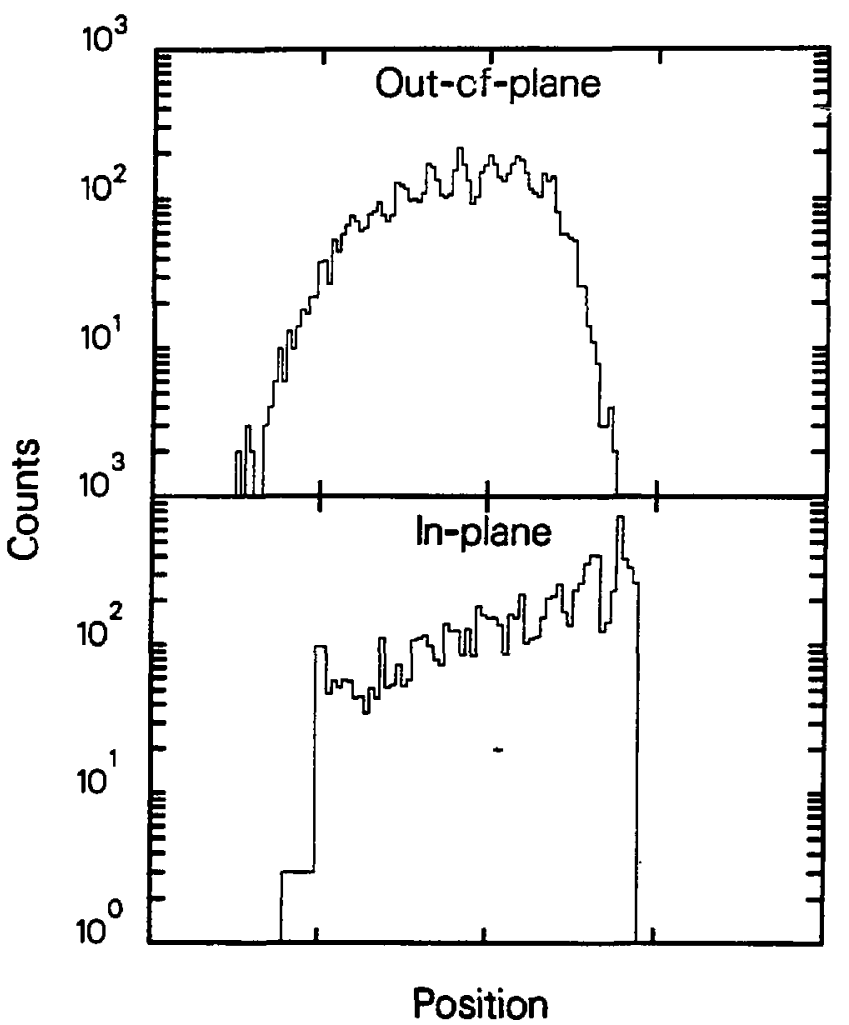

xat $996-2324$

Figure II.6 Corrected and calibrated position spectra from the raw data shown in Figure II.5. 


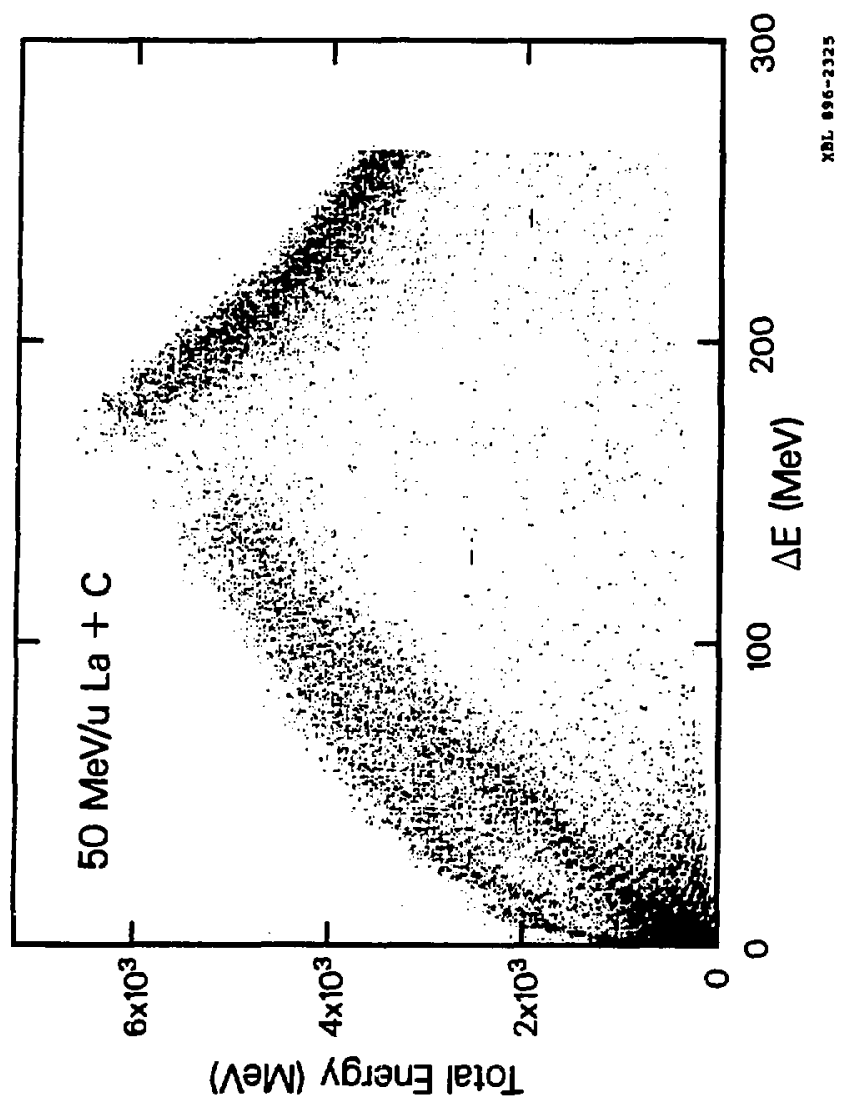

Figure II.7 Total Energy versus $\Delta \mathrm{E}$ (energy loss in the gas ionization detector) spectrum for one of the GaSP telescopes in the $50 \mathrm{MeV} / \mathrm{u}^{139} \mathrm{La}+{ }^{12} \mathrm{C}$ experiment. Each hyperbolic band corresponds to an individual Z-value. The high energy and low energy ridges correspond to fragment emission forward and backward in the source frame (see Figure II.1). The broad band in the upper right portion of the Figure is background from inelastic scattering of beam particles off of the collimator and beam pipe. 


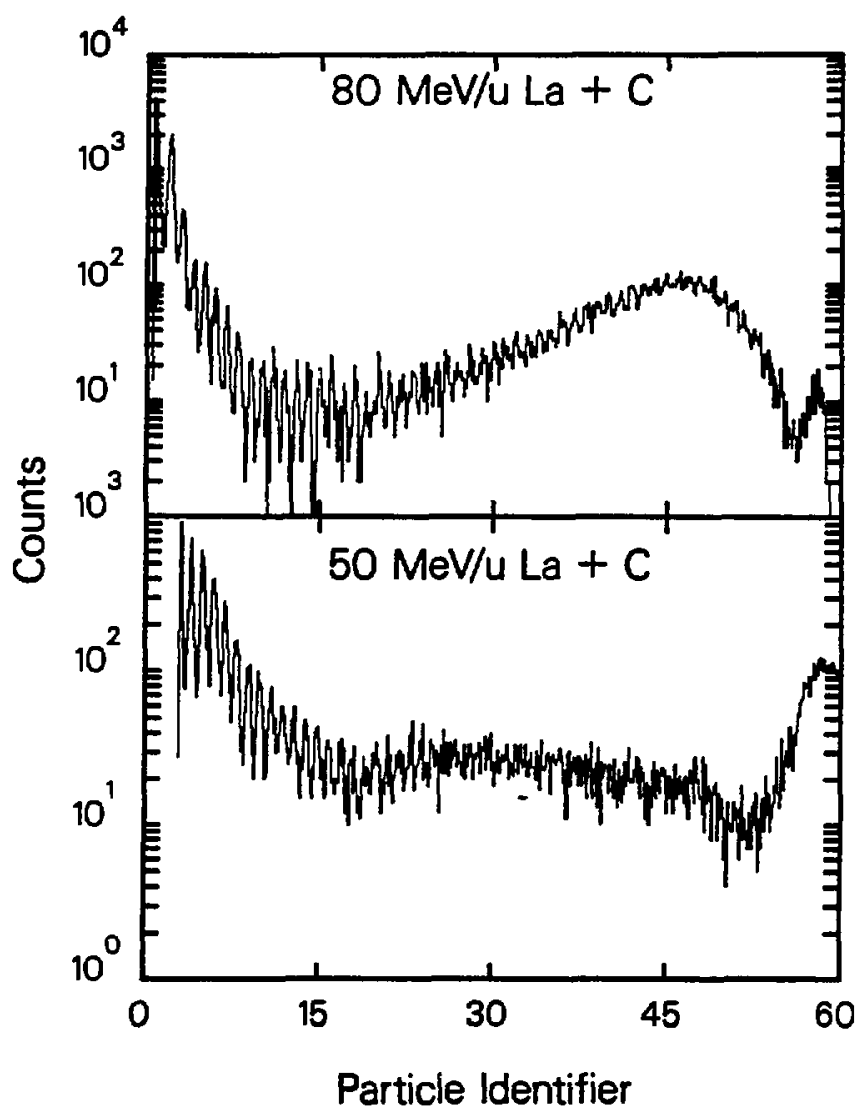

$\operatorname{xoL} 196-2327$

Figure 11.8 Particle identification function for the ${ }^{139} \mathrm{La}+{ }^{12} \mathrm{C}$ reactions at 50 and 80 $\mathrm{MeV} / \mathrm{u}$ (see text). Individual atomic number are resolved up to $\mathrm{Z} \sim 26$ at $50 \mathrm{MeV} / \mathrm{u}$ and up to $Z \sim 50$ at $80 \mathrm{MeV} / \mathrm{u}$. 


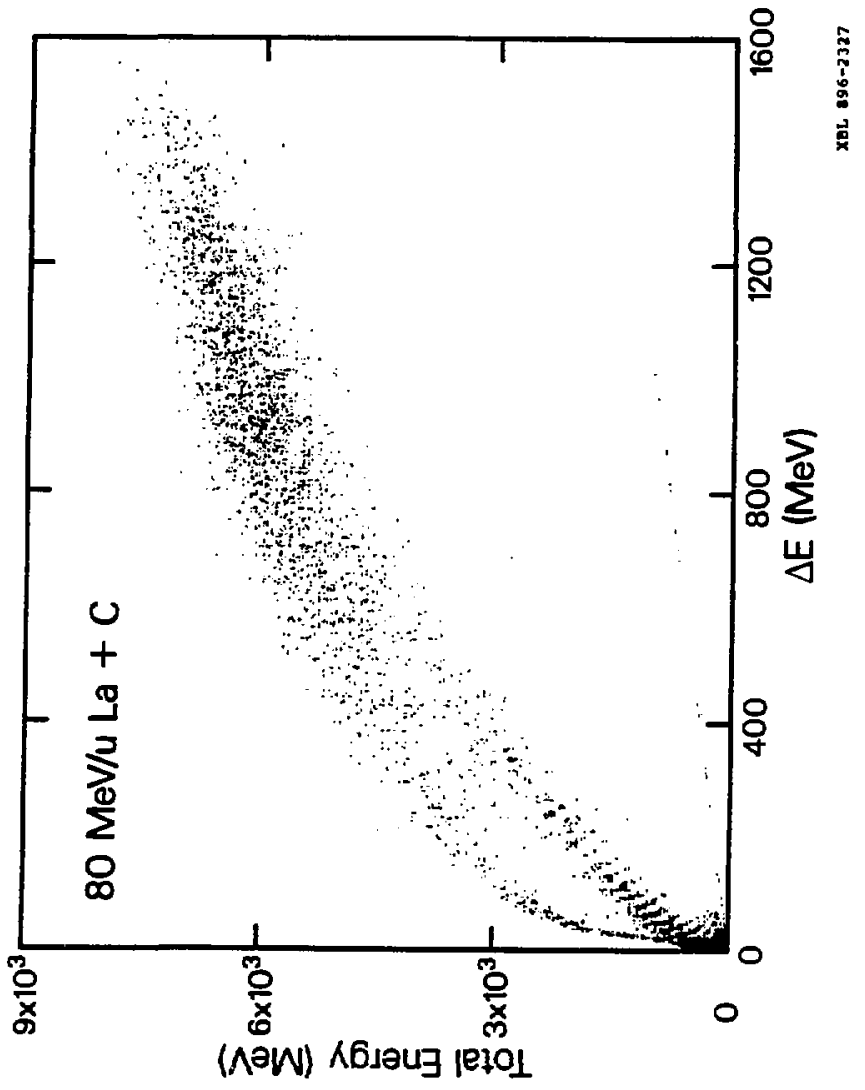

Figure II.9 Total Energy versus $\Delta \mathrm{E}$ (energy loss in the $300 \mu \mathrm{Si}$ ) spectrum for one of the $\mathrm{Si}-\mathrm{Si}(\mathrm{Li})-\mathrm{Si}(\mathrm{Li})$ array telescopes in the $80 \mathrm{MeV} / \mathrm{u}{ }^{139} \mathrm{La}+{ }^{12} \mathrm{C}$ reaction. The bands corresponding to emission forward and backward in the source frame are still apparent at this energy. Note that the spectrum is much cleaner than at $50 \mathrm{MeV} / \mathrm{u}$. There is no observable background from inelastic scattering of beam particles. 


\section{Four Element Telescope for} Reverse Kinematic Studies

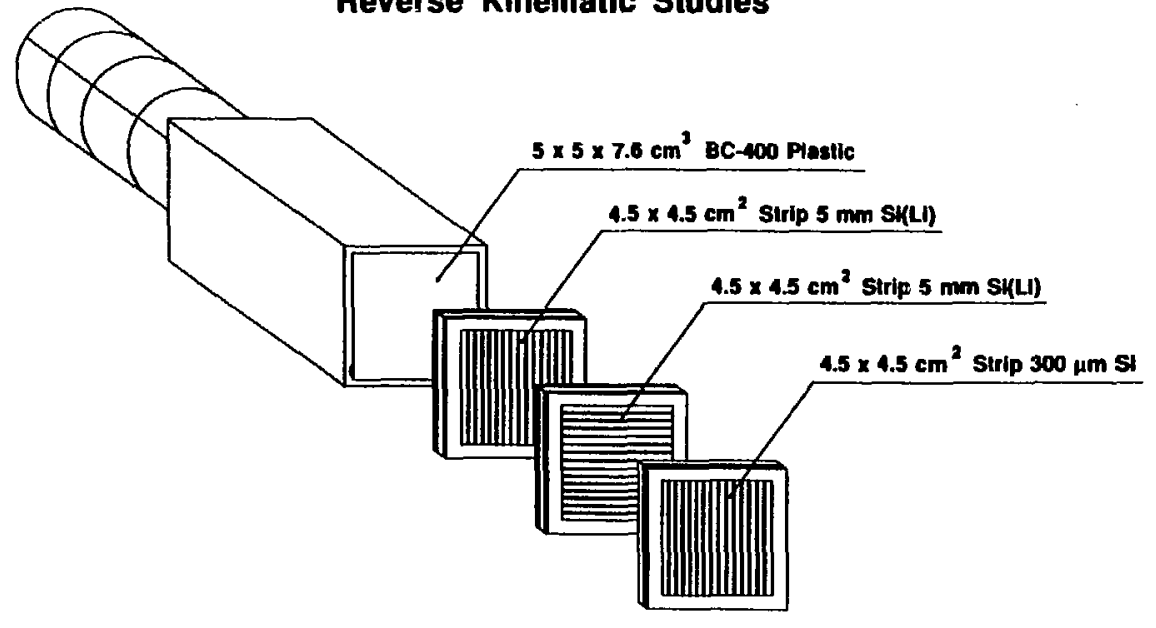

XBL 896-2310 


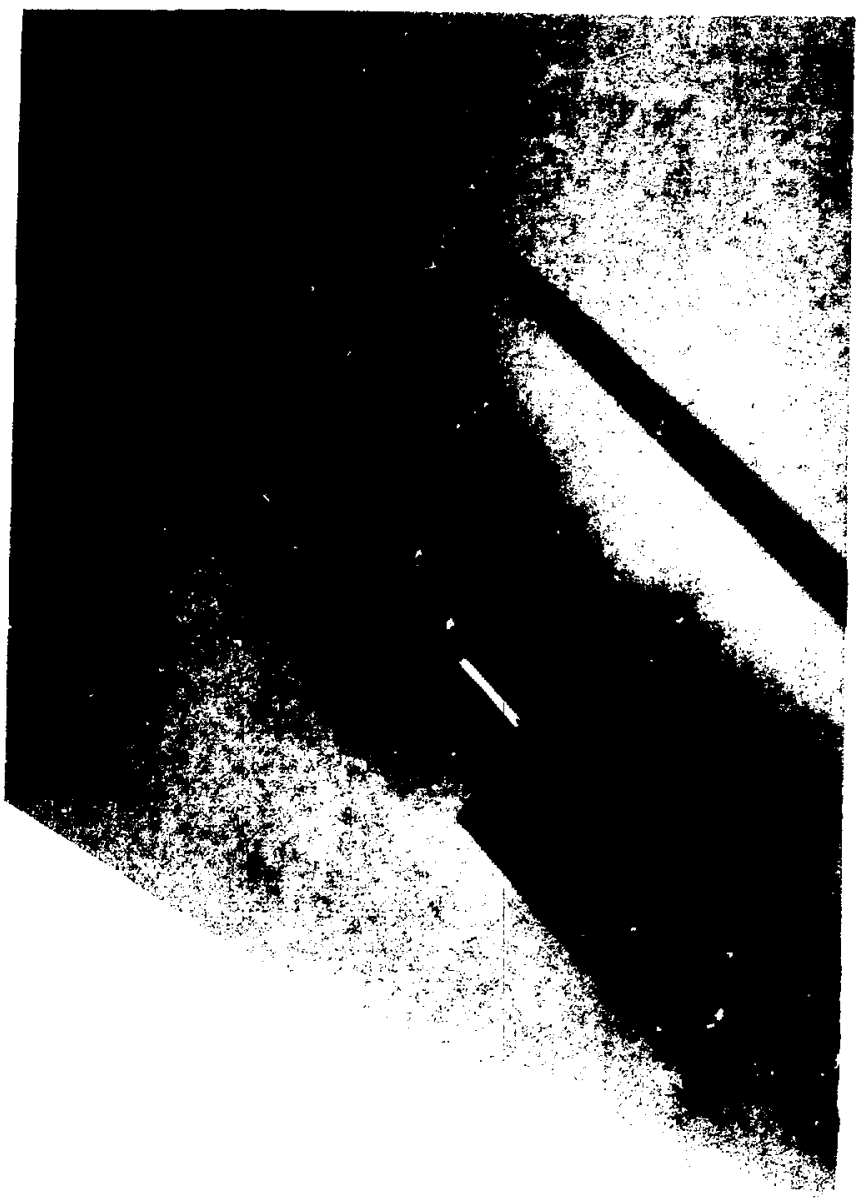

Figure II.11 Photograph of one of the Si-Si(Li)-Si(Li)-plastic telescopes used in the 80 and $100 \mathrm{MeV} / \mathrm{u}$ experiments. The alternating Au and $\mathrm{Pd}$ (dark lines) strifs used for the position determination are visible on the front face of the $5 \mathrm{~mm} \mathrm{Si}(\mathrm{Li})$ detectors. 


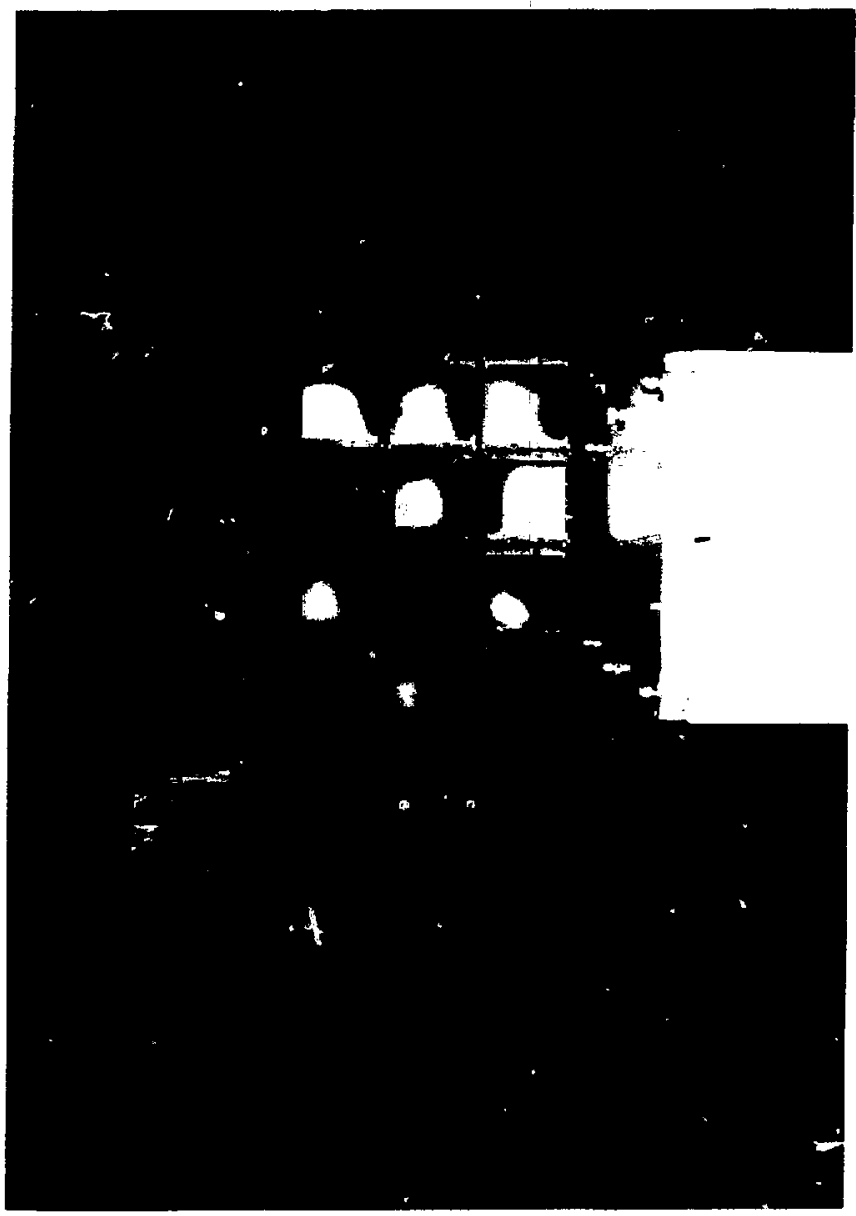

Figure II.12 Photograph of the detector configuration in the 80 and $100 \mathrm{MeV} / \mathrm{u}$ experiments. The detector array was configured in a $3 \times 4$ rectangular geometry, with one telescope missing for the beam. Each telescope was positioned $91 \mathrm{~cm}$ from the target, and subtended approximately $2.8^{\circ}$ both in- and out-of-plane. 
Telescope Logic
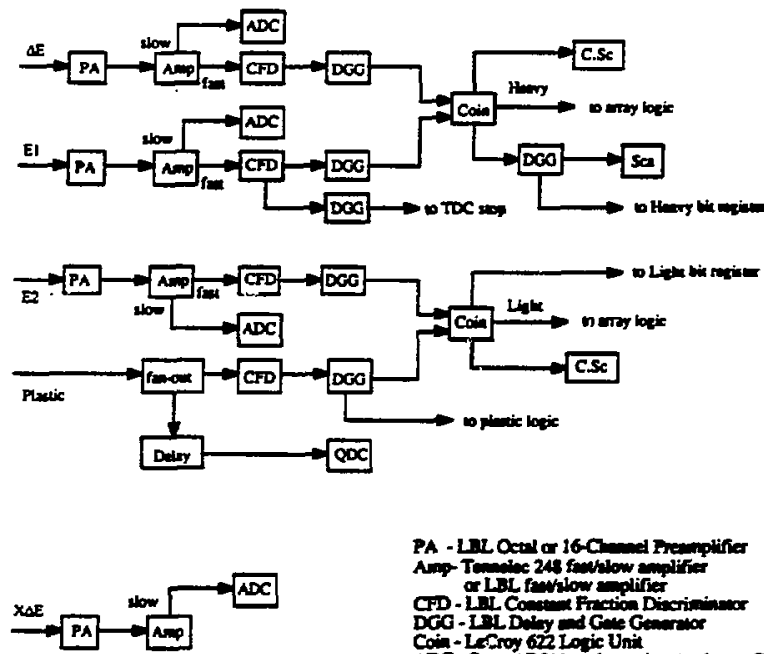

PA - LLC Oct o 16-C and Frempifier Anp Truec 213 fmblow enplifis

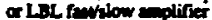

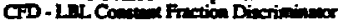

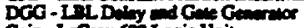
Coin-10roy 62 topic Uni

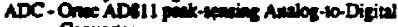
Comines

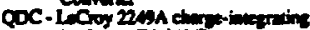
Anto -Dinin Coner

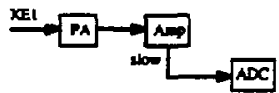

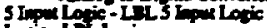

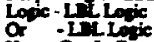

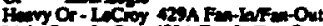

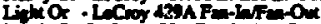

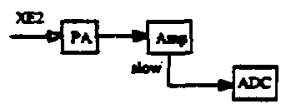

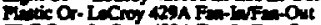

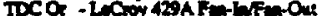

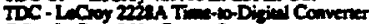
Csc-LCor 2351 Coneseste. Ses - LL V Vind Sein

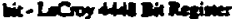

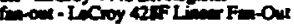

21. 95-2330

Figure II.13(a) Low-level electronics diagram (Telescope Logic) for the 80 and 100 MeV/u experiments. 
Ama Logic

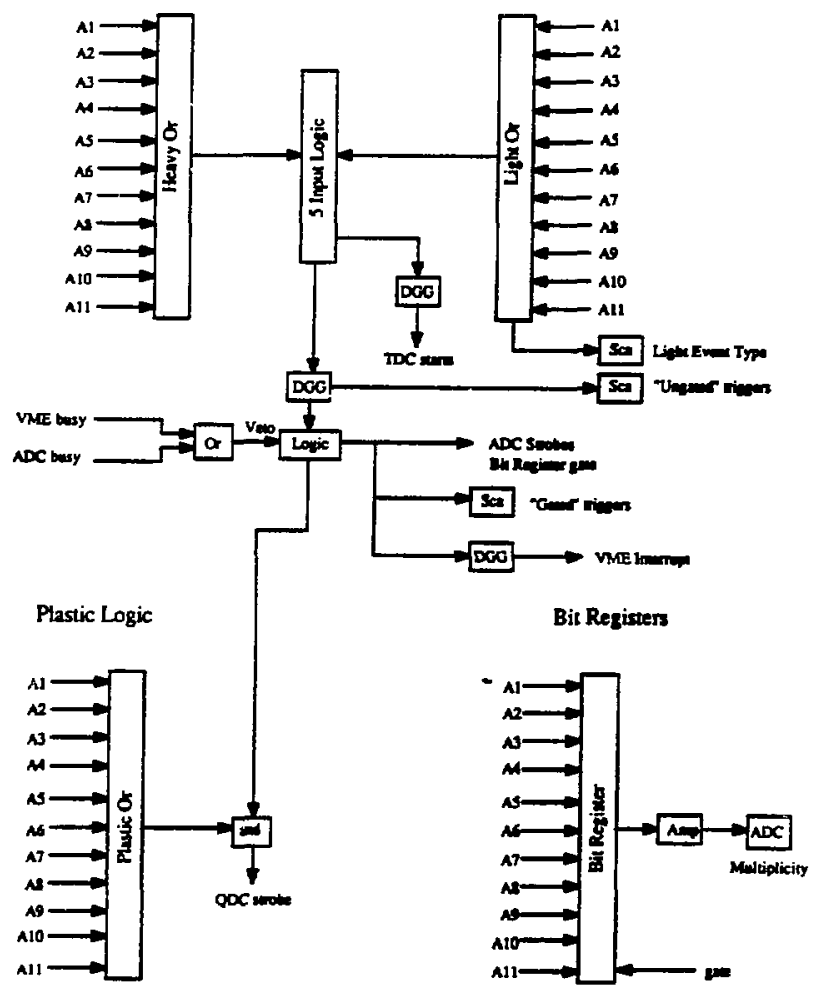

xt a96-2331

Figure II.13(b) High-level electronics diagram (Array Logic) for the 80 and 100 $\mathrm{MeV} / \mathrm{u}$ experiments. The legend is given in Figure II.13(a). 


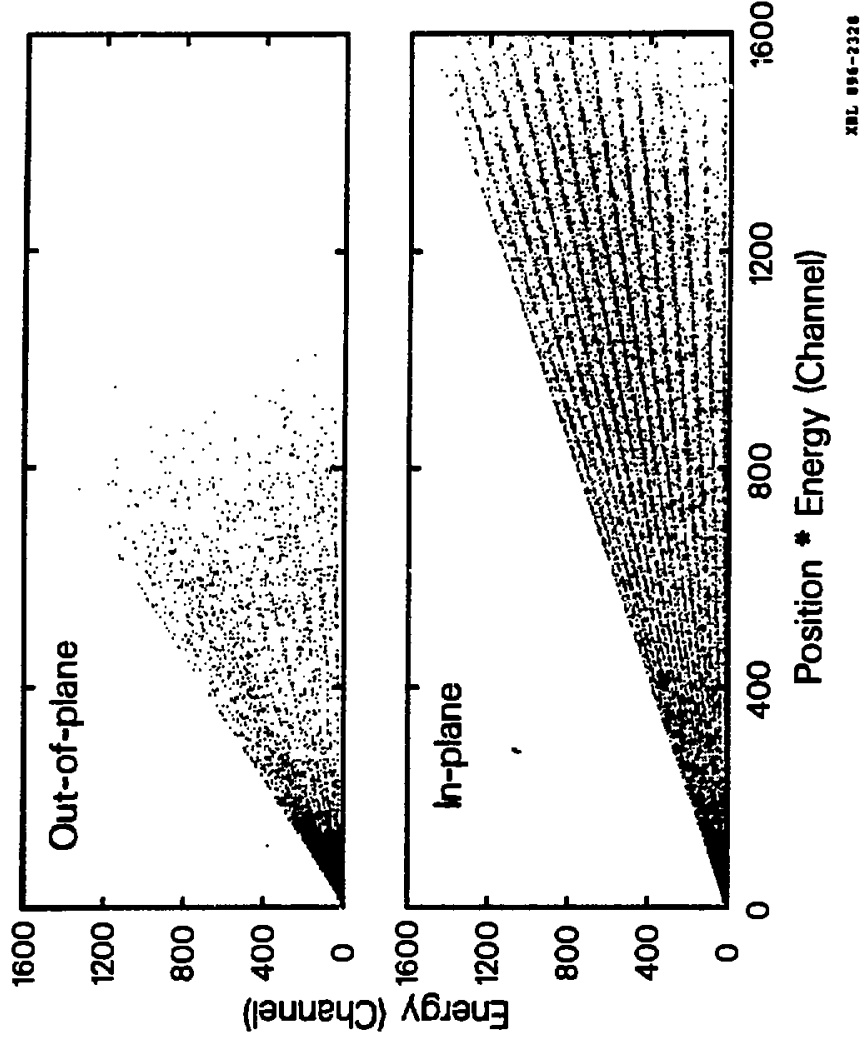

Figure II.14 Raw position spectra for a $300 \mathrm{Si}$ detector (bottom) and a $5 \mathrm{~mm} \mathrm{Si}(\mathrm{Li}$ ) detector (top). In both cases the Energy signal (electrons) is plotted versus the (Positon $x$ Energy) signal (holes). The 15 diagonal lines correspond to the 15 discrete position elements of the devices. 


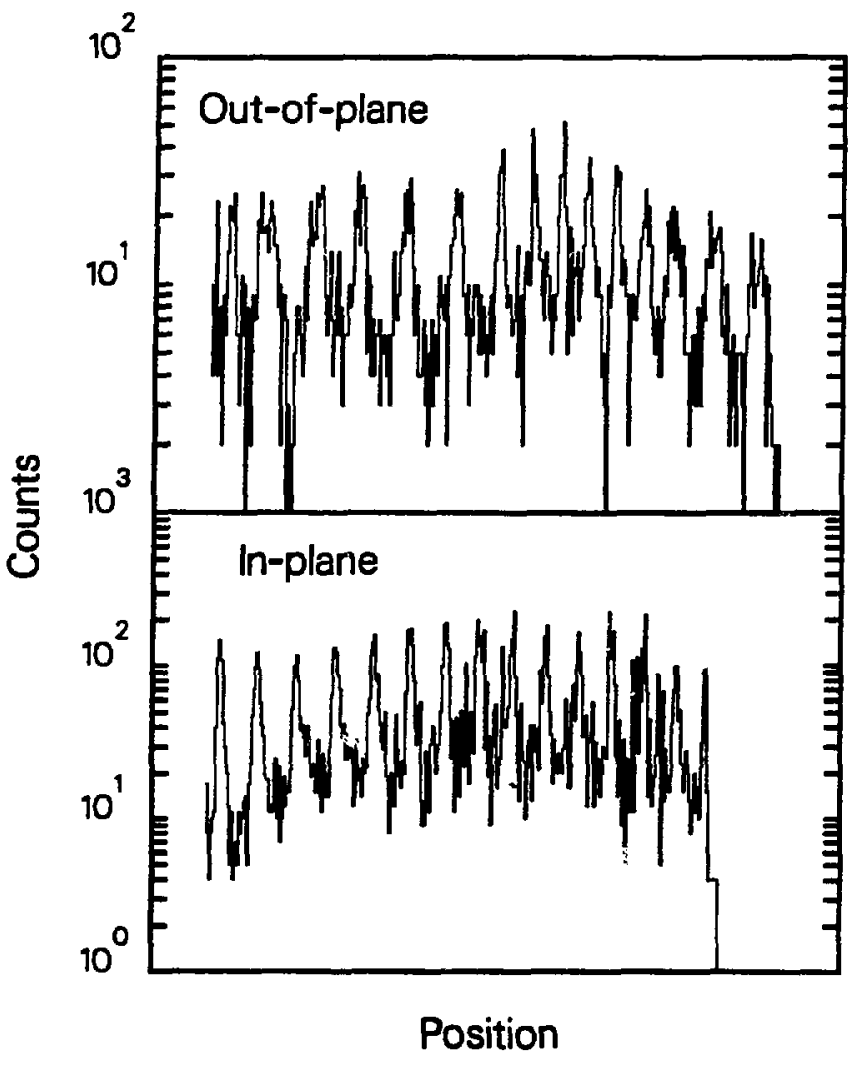

xet $196-2319$

Figure II.15 Calibrated position spectra from the raw data shown in Figure II.14. 


\section{III. ${ }^{139} \mathrm{La}+{ }^{12} \mathrm{C}$ RESULTS}

We will begin the discussion of the ${ }^{139} \mathrm{La}+{ }^{12} \mathrm{C}$ reactions by examining the global nature of the reaction mechanism. We will then turn to the more quantitative results: source rapidities, emission velocities, angular distributions and cross sections. Next, we will investigate the coincidence data in some detail. Finally, we will compare the experimental results with predictions from the incomplete fusion - compound statistical emission model that has successfully explained a large amount of data at lower energy. Throughout the discussion, we will often compare the $50-100 \mathrm{MeV} / \mathrm{u}{ }^{139} \mathrm{La}$-induced reactions to these well-understood lower energy results.

\section{A. Global Results \\ 1. Two-fold Coincidence Events}

Important information about the reaction mechanism can be obrained by examining the two-fold complex fragment coincidence data. Plots of $Z_{1}$ versus $Z_{2}$ and $Z_{1}+Z_{2}$ can tell us whether the reaction proceeds through final states with only two complex fragments, or whether the exit channel is predominantly many body. In the former case we expect that the coincidence charge distributions should peak at values near the total target plis projectile charge, whereas, with a many body final state, we expect the charge distribution to be peaked, if at all, at values significantly less than the total charge in the entrance channel.

Let us consider the linear contour plots of the two-fold $\left(Z_{2}\right.$ versus $Z_{1}$ ) coincidence events at 18,50, 20 and $100 \mathrm{MeV} / \mathrm{u}$ shown in Figure III.1. At bombarding energies $\leq 50$ $\mathrm{MeV} / \mathrm{u}$ the two detectors were placed symmetrically about the beam, thus there is no preferred ordering of $Z_{1}$ or $Z_{2}$. At the higher energies the rietector configuration was asymmetric. For purposes of comparison, the $Z_{1}-Z_{2}$ data in the 80 and $100 \mathrm{MeV} / \mathrm{u}$ reactions have been reflected about the line $Z_{1}=Z_{2}$.

The dashed lines in this figure indicate the total charge of the projectile plus target. 
The figure is striking because, at all bombarding energies, even at $100 \mathrm{MeV} / \mathrm{u}$, the contour plots are dominated by a band of events in which two and only two fragments contain the bulk of the charge. This band broadens and shifts towards a smaller total charge as the bombarding energy increases. Multibody events with more than two heavy fragments fall below the dominant band and become visible at the highest bombarding energy.

Because the bulk of the target and projectile mass is contained in two heavy fragments, the two-body kinematics is well preserved in these reactions. Although the reactions are not strictly binary in the sense that there are only two final fragments, they can still be considered binary in the same way as are low energy fission and deep-inelastic reactions. In these processes, there are two heavy final fragments moving relative to one another with a well-defined velocity determined mainly by the Coulomb repulsion energy between the fragments. The emission of light particles either preceding or following the main binary decay acts mainly to perturb the sharpness of the relative velocity distribution. At higher bombarding energies ( $>20 \mathrm{MeV} / \mathrm{u}$ ), the distribution of sources (in velocity, mass and charge) produced in incomplete fusion processes may also contribute to the broadening of the inclusive velocity distributions.

The two-body nature of the process is also illustrated by the $Z_{\text {Total }}\left(Z_{1}+Z_{2}\right)$ distributions shown in Figure III.2. The total charge distributions in the coincidence events show a well defined peak at all bombarding energies. They show a decrease in $\mathbf{Z}_{\text {Total }}$ and an increase in width with increasing bombarding energy.

The distribution of events along the binary band in Figure III. 1 is strongly related to the detection efficiency for a given charge division. Monte Carlo simulations indicate that the efficiency for detecting symmetric binary decays is much larger at all bombarding energies than that for detecting asymmetric decays. The limited angular coverage of the detectors at $50 \mathrm{MeV} / \mathrm{u}$ is the most biased in this regard. At the other bombarding energies, especially at 80 and $100 \mathrm{MeV} / \mathrm{u}$, the larger solid angle coverage permitted a more efficient detection of asymmetric events and events with three or more complex fragments. 


\section{Z Versus Velocity Diagrams}

The velocity distributions of the complex fragments can also indicate much about the reaction mechanism. The compound binary decay process illustrated schematically in Figure $\Pi 1.1$ predicts that the fragments will be emitted with well-defined velocities. (Of course the sharp emission velocity distribution must be folded into the source velocity distribution, which can be broad in incomplete fusion reactions.) In contrast, products from multifragment emission should always have broad velocity distributions. In order to gain understanding of the emission process, let us examine the fragment velocity distributions using $\mathbf{Z}$ versus velocity contour plots. Details of the efficiency corrections and of the kinematical transformations used to generate many of the experimental distributions are given in Appendix $I$.

Figure III. 3 shows the inclusive reaction products detected at laboratory angles of $3^{*}-8^{*}$ in the $50 \mathrm{MeV} / \mathrm{u}^{139} \mathrm{La}+{ }^{12} \mathrm{C}$ reaction. Linear contours of the Galilean-invariant cross-section $\frac{d^{2} \sigma}{v^{2} d v d \Omega}$ in the $Z$ - velocity plane are shown. The dashed line in this figure is an experimental threshold delimiting the events that stopped in the $2 \mathrm{~mm} \mathrm{Si}(\mathrm{Li})$ detectors from those that punched-through.

Three components of the distribution are visible. The first component consists of heavy fragments $(Z \sim 50)$ moving at well defined velocities slightly smaller than that of the ${ }^{139} \mathrm{La}$ beam. These fragments are a mixture of the heavy partners from very asymmetric complex fragment decay processes and of "classical evaporation residues", which are the residues of hot systems that have lost their excitation energy via the emission of light particles. These fragments have very small velocities relative to the source velocity due to their large mass, and are therefore confined to a small angular region near the beam.

Above $Z=12$ one observes two ridges that meet and merge at about a velocity $\mathrm{V}=$ $0.95 \mathrm{~V}_{\text {beam }}$ and $\mathrm{Z}=35$. This second component has the smallest yield and consists of fragments which we interpret as being produced uniquely in the binary decay of composite 
systems. The two ridges in velocity observed at the same laboratory angle arise from the Coulombic emission of fragments forward and backward in the source frame (see Figure II.1). The Coulomb emission velocity of a fragment in a binary process is given by:

$$
V_{\text {emiss }}=\sqrt{\frac{2\left(M_{S}-M_{1}\right) \text { Ecoul }}{M_{s} M_{1}}}
$$

where $E_{C o u l}$ is the Coulomb energy between the two fragments, $M_{1}$ is the mass of the fragment and $\mathrm{M}_{S}$ is the mass of the composite system. The increasing separation in laboratory velocity between the two ridges with decreasing Z-value is due simply to linear momentum conservation in the Coulomb repulsion process, with the lighter fragments having larger velocities.

The distribution of events along the two Cou' umb ridges is strong evidence for the binary, relaxed nature of the process. There is no filling of the area between the ridges as would be expected for events with higher multiplicities of complex fragments.

The third component has the highest yield, and consists of light $(Z<10)$ complex fragments with velocities significantly smaller than that of the beam. These fragments are the lighter reaction partners in the binary anisotropic process discussed in the Introduction. Unfortunately, at $50 \mathrm{MeV} / \mathrm{u}$ a large background of light fragments from reactions of the ${ }^{139} \mathrm{~L}_{2}$ projectiles in the collimator and beam pipe populate the same portion of the $\mathrm{Z}$ velocity map. Therefore, no more than a qualitative survey of this component is possible.

The continuity of these processes as a function of bombarding energy is exhibited by the similarity of the distributions obtained both at lower bombarding energy [Ch 88a] and in Figures III.4 and III.S for the 80 and $100 \mathrm{MeV} / \mathrm{u}{ }^{139} \mathrm{La}+{ }^{12} \mathrm{C}$ reactions. For these higher energy reactions, the proper Lorentz-invariant cross sections of $\frac{d^{2} \sigma}{\gamma^{4} v^{2} d v d \Omega}$ are shown ${ }^{3}$. An inspection of these diagrams at several laboratory angles shows the same pattern of heavy residues, Coulomb ridges characteristic of relaxed binary decay, and 
anisotropic light complex fragments. The heavy residues dominate the distributions at the smallest laboratory angles. At larger angles where the heavy residues are kinematically forbidden due to their large mass, the non-equilibrium light complex fragments dominate the distributions.

3. $\mathrm{V}_{\|}-\mathrm{V}_{\perp}$ Plots

Another way of portraying the global nature of the emission process is to plot, for a given Z-value, the cross section in velocity space. The Galilean invariant cross section in velocity space is $\frac{\partial^{2} \sigma}{v_{\perp} \partial v_{\perp} \partial v_{\|}}$. However for statistical emission of heavy fragments from systems with high angular momentum, we expect differential cross sections that are isotropic in the reaction plane, $\frac{\partial \sigma}{\partial \Omega} \propto \frac{1}{\sin \theta^{\prime}}$, where $\theta^{\prime}$ is the emission angle in the source frame. To show this reaction plane isotropy, the above invariant cross sections have been multiplied by $\sin \theta^{\prime}$, or equivalently by $v_{\perp}$, since $v_{\perp}=v^{\prime} \sin \theta^{\prime}\left(v^{\prime}\right.$ is the emission velocity in the source frame, and, for statistical emission, is indepindent of $\theta^{\prime}$ in this frame). Therefore, for the $50 \mathrm{MeV} / \mathrm{u}{ }^{139} \mathrm{La}+{ }^{12} \mathrm{C}$ reaction we have plotted the cross sections in velocity space as $\frac{\partial^{2} \sigma}{\partial v_{\perp} \partial v_{\|}}$, which are invariant only with respect to translations of $v_{\|}{ }^{4}$

A schematic representation of the binary emission of complex fragments from an equilibrated source with a laboratory velocity $V_{S}$ is shown in Figure III.6(a). The emission of complex fragments at high angular momentum appears as an isotropic Coulomb ring centered at the arrowbead. In the binary decay process each observed fragment also has a partner emitted at $180^{\circ}$, in general on another ring, with a radius dependent upon the Coulomb repulsion energy and the mass ratio of the fragments. The ring is smeared by pre-equilibrium effects (particle emission and incomplete fusion), sequential evaporation of light paricles, and thermal fluctuations of the Coulomb energy [Mo 75]. The dashed lines in the figure correspond to the limits of the detector acceptance at $3.0^{\circ}$ and $8.0^{\circ}$ in the laboratory. The solid area between these lines is the expected loci of observed events. 
The experimental cross sections (corrected for efficiency) are presented in Figure III.6(b,c,d) for $\mathrm{Z}$ bins of $21-23,24-26$, and $27-29$. The distributions exhibit isotropic rings, strikingly sirnilar to the schematic representation. Arrows 1 and 3 represent the beam velocity and the ve'ocity resulting from complete fusion, respectively. Approximately midway in between, the experimentally determined source velocity is indicated by arrow 2 . The Coulomb rings associated with the different $Z$ bins are centered at approximately the same value of $v_{\|}$, suggesting that all of these fragments have a common origin.

The radii of the experimental distributions correspond to the emission velocities in the source frame. These velocities decrease with increasing fragment charge, in accordance with Coulomb emission systematics given by equation (17) above. The observed independence of the source velocity upon $Z$-value, the decreasing emission velocity with increasing Z-value, and the angular isotropy in the reaction plane are all consistent with complex fragment emission from the binary decay of an equilibrated compound nucleus at high angular momentum.

Cross sections in the rapidity - perpendicular momentum plane $\left(\frac{\partial^{2} \sigma}{\partial Y \partial\left(P_{\perp} / m c\right)}\right)$ are shown for representative Z-values between 6 and 38 for the 80 and $100 \mathrm{MeV} / \mathrm{u}{ }^{139} \mathrm{La}+$ ${ }^{12} \mathrm{C}$ reactions in Figures III.7 and III.8. As at lower energy, the cross sections are distributed along Coulomb rings centered at a constant source velocity. The sharpness of the rings indicates once again that most of the reactions leading to these products are "quasi-binary", and that the two-body kinematics is preserved. These cross sections are invariant only to translations in rapidity ( $Y$ ). Isotropic distributions along the Coulomb rings would indicate isotropic emission in the reaction plane. However, in contrast to the distributions at bombarding energies $550 \mathrm{MeV} / \mathrm{u}$, the distributions at higher energits are no longer isotropic along the rings. They change from backward peaked at $Z<20$, to forward/backward symmetric around $Z=22$, and finally to forward peaked for $Z>26$. 
The anisotropic angular distributions suggest a non-equilibrium emission mechanism at higher energies.

\section{B. Quantitative.Results}

1. Source Rapidities

If all of the fragments have a common origin, we expect no dependence of the source rapidity upon fragment $Z$-value. Should such a dependence be found, it would indicate that the fragments are produced by different mechanisms, or by a range of sources in an incomplete fusion process. Therefore, it is instructive to determine the average source rapidity as a function of fragment Z-value.

The source velocities were extracted from the centroids of the laboratory velocity distributions in the $50 \mathrm{MeV} / \mathrm{u}^{139} \mathrm{La}+{ }^{12} \mathrm{C}$ experiment. In order to facilitate comparisons with the higher energy data, in which relativistic kinematics was used throughout, these extracted source velocities were transformed into rapidities as described in Appendix I.

In Figure III.9 the ratios of the source rapidities to that of the beam for the 50 $\mathrm{MeV} /{ }^{139} \mathrm{La}+{ }^{12} \mathrm{C}$ reaction are plotted as a function of fragment $\mathrm{Z}$-value for $21 \leq \mathrm{Z} \leq 35$. The source rapidities appear constant over this range, indicating the common origin of all of these fragments. The solid line represents the mean source rapidity, which is intermediate between the beam rapidity and the rapidity for complete fusion of the projectile and target (dashed line). The small error bars for each $\mathrm{Z}$ are the statistical errors associated with the extraction of the source velocities; the large single error bar is the possible systematic error from uncertainties in the energy calibration, and the mass parametrization.

In order to extract the source rapidities from the inclusive data at 80 and 100 $\mathrm{MeV} / \mathrm{u}$, the experimental rapidity - perpendicular momentum distributions were Lorentztransformed into an assumed source frame. The centroids of the emission velocity distributions in this frame were determined as a function of the emission angle. The distributions of these centroids as a function of emission angle were then fit to ellipses (see Appendix I). The need to fit the distributions to ellipses, rather than circles, is illustrated 
by Figure III.10, in which the emission velocity in the source frame for $Z=26$ fragments is plotted as a function of the emission angle in this frame. The dashed line is a leastsquares fit of an ellipse to the experimental data. A circular distribution would be indicated by emission velocities that are independent of emission angle. Circular distributions, like those observed at lower energy [Ch 88b], are expected for equilibrium emission from a well-defined source.

The source rapidities at 80 and $100 \mathrm{MeV} / \mathrm{u}$ were extracted from the elliptisai fits to the inclusive data. The source rapidities can also be obtained as the center-of-rnass rapidities of the coincidence events. Figure IIL.9 shows the inclusive and coincidence source rapidities/beam rapidity ratios as a function of fragment $Z$-value for the 80 and 100 $\mathrm{MeV} / \mathrm{u}{ }^{139} \mathrm{La}+{ }^{12} \mathrm{C}$ system. There is a slight systematic disagreement between the rapidities obtained from the inclusive and the coincidence events which is within the experimental uncertainty. As at lower energy, the extracted source rapidities show no dependence upon the fragment $Z$-value.

Figure III.11 shows the major/minor axis ratios of the elliptical fits to the inclusive

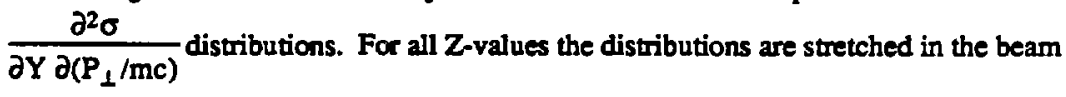
direction. This could be explained by fragment emission from a range of sources with different rapidities corresponding to different amounts of fusion. In conjunction with the previous observation of the independence of the source rapidity on the fragment Z-value, the elliptical distributions suggest that all of these fragments originate from a common distribution of sources.

Source rapidity to beam rapidity ratios extracted from the inclusive and coincidence events for the ${ }^{139} \mathrm{La}+{ }^{12} \mathrm{C}$ system are shown in Figure III.12 as a function of bombarding energy. Within error bars, the ratios show no dependence upon bombarding energy above $50 \mathrm{MeV} / \mathrm{u}$. The solid line is the source rapidity ratio predicted from the momentum transfer systematics [ $\mathrm{Vi} 82, \mathrm{Ga} 82$, Ch 83b, Mo 84, St 85]. The 14, 18, and $50 \mathrm{MeV} / \mathrm{u}$ data points 
are in agreement with the systematics; the 80 and $100 \mathrm{MeV} / \mathrm{u}$ data points show smaller source rapidities (larger momentum transfers) than does the systematics. However, the rapidities above $60 \mathrm{MeV} / \mathrm{u}$ were determined from proton distributions [Ga 82]. Since the barriers for complex fragment decay are much larger than for proton decay, complex fragment emission should, on the average, be associated with events with larger energy deposition. Hence, it is not surprising that the source rapidities (momentum transfers) for complex fragment emission are found to be smaller (larger) than that for proton emission.

2. Emission Velocities

From the radii of the distributions in velocity space we can determine the average emission velocity for each Z-value. At $50 \mathrm{MeV} / \mathrm{u}$ the laboratory distributions were explicitly transformed event-by-event into the source frame determined from the above analysis using the Galilean transformation..$^{5}$ For the 80 and $100 \mathrm{MeV} / \mathrm{u}$ reactions, the emission velocities were determined from the minor axes of the elliptical fits to the rapidity - perpendicular momentum disiributions.

The measured emission velocities are shown in Figure III.13 as a function of fragment $Z$-value. At all energies the velocities decrease with increasing $Z$-value, as expected for Coulombic emission (equation 17). This figure also shows that the emission velocity for a given Z-value gets smaller as the bombarding energy is increased. There are several possible explanations for this. Figures III.1 and III.2 show that the average coincidence charge decreases with increasing bombarding energy. If this charge is lost prior to scission then the complex fragments would be emitted, on average, from systems that have a smaller total charge, so the Coulomb repulsion energy and the corresponding emission velocity would be smaller. If the charge is emitted after scission, the observed secondary fragments are emitted as heavier primary fragments, with smaller emission velocities. In addition as the temperature of the emitting system is increased, the system is predicted to expand, thus lowering the Coulomb repulsion energy in the decay [Ca 83]. (This effect would give rise to a lowering of the conditional barriers to complex fragment 
emission with increased temperature. No evidence for this effect has been seen in the ${ }^{93} \mathrm{Nb}$ $+{ }^{12} \mathrm{C},{ }^{9} \mathrm{Be}$ and ${ }^{139} \mathrm{La}+{ }^{12} \mathrm{C}$ systems at temperatures up to approximately $4 \mathrm{MeV}[\mathrm{Ch} 88 \mathrm{~b}$, Ch 89], as there is no enhancement of the experimental cross-sections above those predicted with zero-temperature barriers. It is therefore likely that a combination of the first two explanations given above accounts for the decrease of emission velocity with increasing bombarding energy.)

Figure III.14 shows the inclusive emission velocities for the three bombarding energies, and the fragment velocities in the center-of-mass frame for 2 -fold coincidence events in the 80 and $100 \mathrm{MeV} / \mathrm{u}$ reactions. Tc calculate the emission velocities from the coincidence events, a general Lorentz-transformation into the center-of-mass frame (not necessarily along the beam direction) was applied (see Appendix I). There is general agreement between the emission velocities calculated from the inclusive and the 2-fold coincidence events.

The lines in Figure III i4 are predictions of the expected emission velocity based upon the Viola fission fragment kinetic energy systematics [Vi 85]. The Viola systematics gives the kinetic energy of the two fission fragments as a function of the $Z$ and $A$ of the fissioning system:

$$
E_{\text {Viol } 2}=0.1189 \frac{Z^{2}}{A^{1 / 3}}+7.3 \mathrm{MeV}
$$

While the Viola systematics has been compiled for symmetric fission, we have generalized it by solving for the radius parameter ro in the equation

$$
E_{\text {Viola }}=\frac{1.44 Z_{1} Z_{2}}{r_{0}\left(A_{1}^{1 / 3}+A_{2}^{1 / 3}\right)}
$$

with $Z_{1}=Z_{2}$ and $A_{1}=A_{2}$. The extracted to was used to calculate the kinetic energy 
released and the fragment velocities for asymmetric decay from equation (19) with $Z_{1} \neq Z_{2}$ and $A_{1} \neq A_{2}$.

To compare predictions from the Viola systematics to the experimental data, corrections must be made for the light charged particle loss. As mentioned above, the loss of light particles could both precede or follow the main binary division.

The emission velocities were calculated using two extreme assumptions. First, the assumption was made that the binary division preceded all of the light particle emission. The measured source velocity was used to infer the charge, mass and excitation energy of the composite system as described below. The emission velocities from this system were calculated from equation (19), and then evaporation corrections were performed for the hot primary fragments using the evaporation code PACE [Ga 80]. The excitation energy of the primary fragments n tetermined from the inferred excitation energy of the compound nucleus and the Q-values for the various binary divisions. The angular momenta $\left(\mathrm{J}_{1}\right)$ of the binary decay products were calculated in the sticking limit, $J_{1}=\left(I_{1} / I_{0}\right) J_{0}$, from the spin distributions used in the statistical model calculations described below. Here $\mathrm{J}_{1}$ and $\mathrm{J}_{0}$ are the spin of the fragment and the compound nucleus, and $I_{1}$ and $I_{0}$ are the moments of inertia of the fragment and of the composite system The moment of inertia of the composite system is $I_{0}=I_{1}+I_{2}+\mu R^{2}$, where $I_{1}$ and $I_{2}$ are the moments of inertia of the fragment and its decay pariner, $\mu$ is the reduced mass of the system, and $R$ is the separation distance between the fragments. The dashed lines in Fig. III.14. are the emission velocities calculated for the assumption of post-scission light charged particle emission.

The emission velocities were also calculated by assuming that all of the light particle emission preeeded the binary decay. A system with the average charge measured from the $\mathbf{Z}_{1}+\mathbf{Z}_{2}$ coincidence distributions was assumed to decay. Statistical model calculations were performed to estimate the post-evaporative masses associated with the measured charges. The calculated masses were essentially the same as predicted by the $A=2.08 \mathrm{Z}+$ $0.0029 \mathrm{Z}^{2}$ mass parametrization [Ch $88 \mathrm{a}$ ]. The extended Viola systematics was used as 
above to calculate the emission velocities from the residual systems (solid line in Figure III.14).

The excellent agreement between both calculations and the data for intermediate Zvalues illustrates the complete relaxation of the entrance channel kinetic energy in the decay process. Better agreement between data and calculation has been obtained for the smaller Z-values by taking into account angular momentum effects [Ch 89]. The heavier fragments are likely to be emitted from systems that are heavier than the average values assumed in the calculations, and thus they have larger emission velocities than predicted. The two predicted emission velocity distributions (solid and dashed lines) were found to be essentially identical over the Z-value range measured in the experiments. This indicates that the emission velocities are insensitive to small variations of the total mass of the system at scission.

Figure III.15 shows emission velocity spectra for $Z=6$ fragments from the reaction of $80 \mathrm{MeV} / \mathrm{u}{ }^{139} \mathrm{La}+{ }^{12} \mathrm{C}$ at several angles in the source frame. These spectra were generated with an event-by-event Lorentz transformations of the inclusive data into the measured source frame. While the yields are approximately a factor of 10 larger for backward emission than for forward, the shapes of the distributions are very similar. This is in stark contrast to data obtained with the same system at $18 \mathrm{MeV} / \mathrm{u}$ [Ch 89], and with many others systems in the $15-40 \mathrm{MeV} / \mathrm{u}$ bombarding energy regime. In these data the emission velocity spectra of the light fragments have high energy tails at forward emission angles in normal kinematic reactions, or backward angles in reverse kinematic reactions. These tails indicate the incomplete relaxation of the entrance channel kinetic energy, and are evidence for the deep-inelastic production mechanism. At $80 \mathrm{MeV} / \mathrm{u}$, however, the emiscion velocities for fragments near the target $Z$-value are completely relaxed over the entire angular region.

3. Angular Distributions

Statistical compound emission requires the relaxation of the angular degrees of 
freedom prior to the decay process. The resulting fragment angular distributions are forward-backward symmetric. The two limiting cases are: the emission of light particles, such as neutrons and protons, which is nearly isotropic (do/d $\Omega \propto$ constant), and heavier fragment emission at high angular momentum, such as detected in this study, which is nearly isotropic in the reaction plane, thus $d \sigma / d \Omega \propto 1 / \sin \theta$, or $d \sigma / d \theta \propto$ constant [Va 73].

While the limited angular region spanned and the poor statistics preclude the determination of complete angular distributions for the $50 \mathrm{MeV} / \mathrm{u}$ data, the ratio of the differential cross sections forward and backward of $90^{\circ}$ in the source frame can be investigated at all bombarding energies. The forward/backward ratios of $d \sigma / d \theta$ in this frame are shown in Fig III.16. The solid lines are linear least squares fits to the data. For the $50 \mathrm{MeV} / \mathrm{u}$ data, the mean forward/backward ratio is $1.06 \pm 0.10$ for $\mathrm{Z}$-values in the range of 22-35, and is virtually independent of the fragment atomic number. Thus, the fragment yields are symmetric about $90^{\circ}$, as required by the compound nucleus emission process.

In contrast to the $50 \mathrm{MeV} / \mathrm{u}$ reaction, at the larger bombarding energies the forward/backward ratios increase smoothly with increasing Z-value. The rate of change of the forward/backward ratio with Z-value is slightly larger at $100 \mathrm{MeV} / \mathrm{u}$ than at $80 \mathrm{MeV} / \mathrm{u}$. The anisotropy of the fragment distributions at 80 and $100 \mathrm{MeV} / \mathrm{u}$ strongly suggests that non-equilibrium processes account for much of the complex fragment yield.

The counting statistics and angular coverage are such in the 80 and $100 \mathrm{MeV} / \mathrm{u}$ reactions that angular distributions can be presented. Figure III.17 shows inclusive angular distributions for _epresentative Z-values. For symmetric fragments with Z-values approximately half of the average coincidence charge $\left(Z=\frac{1}{2}\left(\overline{\left.Z_{1}+Z_{2}\right)}=22-24\right)\right.$ the distributions are flat and consistent with statistical emission. However, for lighter and heavier Z-values, the distributions show strong anisotropies. In contrast to angular distributions at lower bombarding energy, which were interpreted as consisting of both a 
flat compound nucleus component and an exponential deep-inelastic component, the angular distributions of asymmetric products at these larger bombarding energies do not exhibit flat regions.

Monte Carlo simulations were periormed to investigate if a range of isotropic sources could give rise to the observed anisotropic angular distributions. Anisotropic angular distributions could be generated by assuming that the source mass depended upon the source velocity. This is not an unreasonable assumption for incomplete fusion products. However, the resulting anisotropic angular distributions were still relatively independent of the fragment $Z$-value, and the evolution from backward to forward peaking was not observed. Thus it was concluded that the experimental angular distributions originate from an intrinsically anisotropic emission process.

An interesting speculation about the nature of the anisotropic yield relates these fragments to the dynamical transition from the incomplete fusion regime to the fireball regime.

In the fireball model $[\mathrm{Bo} ; 3$, We 76, Go 77$]$, the nuclear matter is divided into three geometrical regions - the projectile spectator, the target spectator, and the fireball or the region in which the nucleons in the target and spectator overlap. In an instantaneous projectile-target interaction the thermal energy per nucleon in the fireball is much larger than the nucleon binding energy. Thus it is likely that the participants in the fireball region would be emitted entirely as nucleons. The complex fragments that are observed in asymmetric reactions at $\mathrm{E} / \mathrm{A} \gg 100 \mathrm{MeV} / \mathrm{u}$ are emitted very nearly isotropically [Wa 83 , Bo 87, Ye 88]. These products have been explained as resulting from the decay of the excited target spectator in a two-step reaction mechanism [Po 71].

At the other extreme, when the relative velocity is not large enough for the fireball to decouple quickly from the spectator fragments, then the region of high excitation can expand ar. $\mathrm{d}$ cool. In the limit of complete equilibration we have the incomplete and finally complete fusion processes seen at lower energy. 
In some bombarding energy region, which will öepend upon the target-projectile combination, we should observe the transition from the incomplete fusion mechanism to the fireball mechanism. This transition may not be particularly sharp as it will also depend upon impact parameter. A geometrical-kinematic model that gives some insight into this process is described in Appendix II. Just below the threshold bombarding energy, when the fireball is barely captured by the projectile-spectator, the product nucleus may have an elongated shape. If this product nucleus subsequently decays before the shape degree of freedom is able to relax, the fragment should point towards the light spectator and the angular distributions could be influenced by the dynamical interaction process. Similarly, just above the threshold bombarding energy, the fireball and the target spectator will have a relative velocity determined mainly by their munal Coulomb repulsion. In either of these scenarios, the rather long interaction time between the fireball and the spectator fragment could allow for a large amount of energy thermalization and mass transfer, and give rise to the observed anisotropic complex fragments.

\section{Cross Sections}

The measured differential cross sections at $50 \mathrm{MeV} / \mathrm{u}$ were integrated over $180^{\circ}$ assuming the $1 / \sin \theta$ angular distribution required by compound nucleus emission at high angular momentum. The angle-integrated cross sections for the 80 and $100 \mathrm{MeV} / \mathrm{u}$ reactions were extracted from quadratic fits to the measured differential cross sections (solid lines in Figure III.17). The angle-integrated cross sections as a function of fragment Z-value for the ${ }^{139} \mathrm{La}+{ }^{12} \mathrm{C}$ system at 18 [Ch 89$], 50,80$ and $100 \mathrm{MeV} / \mathrm{u}$ are shown in Figure II!.18.

The charge distributions for fragments of $8<\mathrm{Z}<40$ at $18 \mathrm{MeV} / \mathrm{u}$ [Ch 89] are consistent with the statistical emission from a system above the Businaro-Gallone transition point [Bu 55]. There is a maximum in the yield at symmetry due to a minimum in the potential energy surface above this point (see Figure I.1). Between 18 and $50 \mathrm{MeV} / \mathrm{u}$ the yields decrease and the charge distribution becomes flatter. 
The decrease in the cross sections for symmetric products as the bombarding energy is increased above $20 \mathrm{MeV} / \mathrm{u}$ is contrary to what is generally expected. If the nuclear temperature increases with increasing bombarding energy, we expect high barrier complex fragment emission to compete more favorably with low barrier light particle evaporation (see equation 6). Additionally, at larger bombarding energies multiple emission of complex fragments (either sequentially or simuitaneously) should become likely. As in another system $\left({ }^{93} \mathrm{Nb}+{ }^{27} \mathrm{Al}[\mathrm{Ch} 88 \mathrm{~b}]\right)$, we can attribute the observed decrease in the symmetric cross sections to the onset of the incomplete fusion process above $=20 \mathrm{MeV} / \mathrm{u}$. The symmetric fission-like events are likely to be produced in the more central collisions, whereas the light non-equilibrium fragments are likely to be produced at larger impact parameters. With increasing bombarding energy the range of impact parameters that gives rise to incompletely equilibrated products should increase due to dynamical effects (see Appendix II). This increase in the non-equilibrium cross sections has been experimentally observed between 20 and $50 \mathrm{MeV} / \mathrm{u}$ [Fi 89]. The flattening of the charge distribution with increasing bombarding energy can be explained by the increase in temperature of the system, which tends to make all of the decay channels more equally probable (equation (6) above).

From 50 to $80 \mathrm{MeV} / \mathrm{u}$ the yields increase and the charge distribution evolves into a $\mathrm{U}$-shape. The symmetric shape of the charge distribution is another indication of the predominantly binary nature of the decay process. A large number of multibody events should give rise to monotonically decreasing cross sections, or to cross sections that remain flat with increasing Z-value. Although this U-shape is incompatible with statistical emission from a system beyond the Businara-Gallone point, it is tot inconsistent with statistical emission from a system that has lost a large amount of charge, or angular momentum, or both, prior to the emission process. Thus we can draw no conclusions about the equilibrium or non-equilibrium nature of the process solely from the shape of the charge distributions. 
From 80 to $100 \mathrm{MeV} / \mathrm{u}$ the yields increase again. It is not clear whether the distribution is still $\mathrm{U}$-shaped at $100 \mathrm{MeV} / \mathrm{u}$ or whether it has flattened out, indicating a significant amount of multibody decay.

\section{Coincidence Data}

1. Total Charge and Rapidity Dependence on Fragment Multiplicity

From Figure III. 2 we see that the peak in the disuribution of total detected charge in the 2-fold coincidence events shifts to smaller values and the width increases as the bombarding energy is increased. The question arises concerning the nature of the missing charge. Is it due to an undetected light complex fragment(s), or due instead to larger multiplicities of protons and alpha particles? We can answer this question by examining the tocal charge distributions as a function of the complex fragments multiplicity (Figure III.19). This figure indicates that the total charge distributions are insensitive to the complex fragment multiplicity at both 80 and $100 \mathrm{MeV} / \mathrm{u}$. This strongly suggests that all of the missing charge is the form of undetected light particles. It would be very interesting to determine whether the lost light charged particles are evaporated or emitted prior to equilibrium. The number of evaporated particles would give a good indication of the amount of (thermalized) excitation energy in these systems.

A similar comparison of the rapidity in the $\mathrm{CM}$ frame as a function of the multiplicity of complex fragments also shows no dependence (Figure III.20). These two results are strong evidence for the common origin of all of the complex fragments. The 3 and 4 fold coincidences do not pick out a special class of events with larger momentum transfers or excitation energies.

\section{Charge Loss and Widths}

The average charge loss from 2 -fold coincidence events as a function of the $Z$-value of one of the fragments is presented in Figure III.21. Although the charge loss increases with increasing bombarding energy for all mass asymmetries, the shapes of these distributions are very similar. The charge loss distributions go through minima near 
symmetry, rise again for heavier fragments, before finally decreasing for the heaviest detected fragments at 80 and $100 \mathrm{MeV} / \mathrm{u}$.

From the measured source rapidity/beam rapidity ratios, which are independent of bombarding energy, the atomic number of all of the composite systems has been inferred to be -60 . Corrections for light charged particle evaporation, assuming post-scission emission, have been performed with the evaporation code PACE $[\mathrm{Ga} 80]$ as described above. The prediction of the charge loss distribution from the PACE simulations is shown by the dashed lines in the figure. The solid lines show statistical model calculations that includes both light charged particle emission and complex fragment emission (see below). Both models underpredict the measured charge loss somewhat, although the PACE simulations do a slightly better job at the larger bombarding energies.

Although the absolute magnitudes of the data and the statistical model predictions differ slightly, the shapes of the charge loss distributions are similar. This similarity of shapes at all bombarding energies is evidence for the statistical nature of the light charged particle loss. Light complex fragments tend to evaporate more charged particles per unit excitation energy than do heavier fragments, due to their smaller Coulomb barriers for charged particle emission. The experimental distributions pass through minima for symmetric decay in which no light complex fragments are emitted, and then rise again for larger Z-values, which are enitted in coincidence with a light complex fragment. Hence, it appears that the potential energy influences the light charged particle emission process.

At the largest $Z$-values the charge loss becomes smaller once more. The observed differences between the experimental and theoretical distributions for these Z-values can be ascribed to detector efficiency effects and to the experimental distribution of sources (in contrast with the "average" source used for the statistical decay calculations). The largest Z-values may be associated with products from peripheral reactions with smaller excitation energies.

The average charge loss of coincidence events is shown as a function of the center- 
of-mass energy for the ${ }^{139} \mathrm{La}+{ }^{12} \mathrm{C}$ system in Figure $\mathrm{III} 22(\mathrm{~b})$. The slope of the linear least-squares fit line (solid line) is about $50 \mathrm{MeV}$ per charged particle. This value is very close to that observed experimentally for a range of sources in the ${ }^{139} \mathrm{La}+{ }^{64} \mathrm{Ni}$ system [Co 89], and, as shown in Figure III.21 above, is in fairly good agreement with statistical emission calculations for these systems. The solid line in Figure III.22(b) does not pass through zero, presumably because only neutrons are emitted from the neutron-rich primary fragments at low excitation enurgy.

Figure III.22(a) shows the widths of the rapidity and momentum distributions from the 2-fold coincidence events. The width of the rapidity distribution is slightly larger than the other widths, and more significantly, it increases more steeply with center-of-mass energy. This observation accounts for the change from nearly circular to elliptic distributions in velocity space with increasing bombarding energy.

We have attempted to reproduce an elliptical rapidity - $P_{\perp} /$ me distribution by assuming that a range of sources with the source rapidity distribution given in Figure III.20 emits fragments with a constant emission velocity. The solid line in Figure $\mathrm{III} .10$ is the result of a simulation for $Z=26$ fragments in the $80 \mathrm{MeV} / \mathrm{u}{ }^{139} \mathrm{La}+{ }^{12} \mathrm{C}$ reaction. This simulation shows that the observed elliptical distributions are due to the extended source velocity distribution, rather than to an emission velocity dependence upon the emission angle.

The momentum distributions in- and out-of-plane in Figure III.22(a) should, in principle, be identical. Although the rates of change of the widths with center-of-mass energy are identical, the difference in magnitude is most likely due to systematic errors introduced by the (asymmetric) detector geometry, the beam spot size, and beam divergence.

3. Monte Carlo Calculations

In order to investigate the biases associated with the detector configuration, a Monte Carlo simulation of the decay process at $50 \mathrm{MeV} / \mathrm{u}$ has been performed [Ch 88a]. In this 


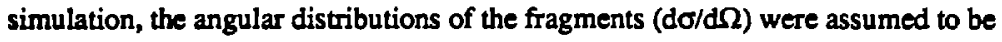
proportional to $1 / \sin \theta$ in the frame of the binary decay, as required by compound nucleus emission at high angular momentum. The first and second moments of the experimental emission velocity distributions were used to generate emission velocity distributions for each Z-value. The velocities in the source frame were then transformed (nonrelativistically) to the laboratory system using the measured source velocity. The beam spot size and beam divergence were incorporated into the simulation, since these quantities have only a negligible effect on the emission velocity, but can have a significant effect on the coincidence efficiency. Finally from the experimental detector geometry, the ratio of binary events, in which both heavy partners were detected, to inclusive events, in which at least one of the fragments was detected, was determined.

Figure III.23 shows the comparison of the experimental coincidence/inclusive ratios to those calculated with the Monte Carlo simulation. The average deviation between the data and the simulation in the range of $21 \leq \mathrm{Z} \leq 35$ is less than $3 \%$. This excellent agreement is additional evidence for the binary nature of the process. If there were a significant fraction of multibody events we would expect deviations from the predictions of the simulation. These deviations could act either to decrease the coincidence/inclusive ratio, if the experimental configuration is optimized for the two-body kinematics, or to increase the ratio, if the detector geometry is not optimized for the two-body kinematics [Ch 88a]. D. Model Calculations

At $18 \mathrm{MeV} / \mathrm{u}$ the experimental results of the ${ }^{139} \mathrm{La}+{ }^{12} \mathrm{C}$ reaction have been explained by complete fusion followed by the sequential compound statistical decay of the equilibrated fusion product [Ch 89]. In the following we will compare our experimental results to the incomplete fusion - compound statistical emission model, which has successfully explained the results of asymmetric reactions at bombarding energy of 25 - 40 $\mathrm{MeV} / \mathrm{u}[\mathrm{Ch} 86, \mathrm{Ch} 88 \mathrm{a}]$. 


\section{Incomplete Fusion}

The source rapidities, as determined from both inclusive and coincidence events, indicate that in the bombanding energy range of this work ( $250 \mathrm{MeV} / \mathrm{u})$ complete fusion has given way to incomplete fusion, in agreement with many previous studies [Vi 82, Ga 82, Ch 83b, Mo 84, St 85]. Two different models of the incomplete fusion process have been applied. A simple prescription is to calculate the mass transfer, momentum transfer, and excitation energy of the incomplete fusion product from the measured source rapidity by assuming that all of the beam momentum is given to the incomplete fusion product and that the lighter reaction partner is sheared in the incomplete fusion process. According to the model that is introduced below and developed in Appendix II, the lighter reaction partner is more easily able to be sheared than the heavier partner.

Relativistically (see Appendix I) we have:

$$
A_{\text {Trans }}=\frac{P_{\text {berm }}}{u \sinh (Y)}-A_{P} \text {, }
$$

where $A_{p}$ is the projectile mass, $P_{\text {bean }}$ is the beam momentum, $Y$ is the experimentally determined source rapidity, $u$ is the energy equivalent of one atomic mass unit (931.5 $\mathrm{MeV} / \mathrm{c}^{2}$, and $\mathrm{A}_{\mathrm{Tr}}$ is the calculated mass transferred from the ${ }^{12} \mathrm{C}$ target to the ${ }^{139} \mathrm{La}$ projectile.

This determination of the mass transferred to the projectile allows the deposited excitation energy to be calculated as:

$$
E^{*}=E_{\text {beam }}-E_{I F}+Q .=E_{\text {beam }}-u \cosh (Y)\left(A_{P}+A_{\text {tran }}\right)+Q
$$

$E_{\text {beam }}$ is the beam energy, $E_{\text {IF }}$ is the energy of the incomplete fusion product, and $Q$ is the ground state Q-value of the incomplete fusion process. The Q-values for the incomplete 
fusion reactions were calculated to be $-20 \mathrm{MeV}$ assuming two fragments in the exit channel (incomplete-fusion product and the target remnant). The $Q$-values could range as low as $45 \mathrm{MeV}$ if, instead, the non-fusing portion of the ${ }^{12} \mathrm{C}$ target escapes as single nucleons. However, the $Q$-value is a small fraction of the total excitation energy in these reactions. Rather than assuming that the non-fusing portion of the ${ }^{12} \mathrm{C}$ target has zero laboratory velocity, a more sophisticated model of the process can be employed. A simple geometrical - kinematic model (Appendix II) has been developed that calculates the drag induced upon the target remnant during incomplete fusion. This drag comes from the impulse associated with creating new nuclear surface in the shearing process. In this model a similar source velocity will give rise to slightly smaller mass transfers than with the zero spectator momentum model described above, due to the drag of the target remnant on the incomplete fusion product. This model predicts mass and momentum transfers, source rapidities and excitation energies as a function of impact parameter.

We have used the geometrical-kinematic model predictions for mass transfer, momentum transfer, and excilation energy at the impact parameter at which the model predicts the experimental source rapidities. This may not be a bad assumption, particularly for incomplete fusion reactions, since the emission process is strongly dependent upon the angular momentum of the emitting system, and a small impact parameter range may account for the bulk of the complex fragment cross sections (see Fig III.24). We do not expect this geometrical-kinematic model to give more than a schematic picture of the incomplete fusion process, but it improves upon the zero spectator momentum model which does not take the nuclear force into account at all.

The model predictions for momentum transfers calculated in a frame moving along with the projectile ("normal kinematics"), mass transfers, and excitation energies are shown in Table III.1. At all energies, the two models give similar results for mass and momentum transfers because the target spectator is not massive enough to perturb the incomplete fusion product significantly. The small differences in the predicted excitation 
energies at 80 and $100 \mathrm{MeV} / \mathrm{u}$ arise because relativistic kinematics have not been incorporated into the geometrical-kinematic model. At bombarding energies up to 50 $\mathrm{MeV} / \mathrm{u}$ the two models predict similar excitation energies.

2. Statistical Decay Calculations

The statistical decay calculations were performed using the Monte Carlo code GEMINI [Ch 88b]. The decay widths for the emission of heavy fragments $(Z \geq 3)$ are calculated using the transition state formalism [Mo 75] as described in the introductory section. The relevant equation is (3). The decay widths for the emission of light particles (Z $\leq 2)$ are caiculated using the Hauser-Feshbach formalism [Ha 52]. Details of this calculation, including expressions for the transmission coefficients and the strong absorption radii are described in [Ch 88b].

The quantitative calculation of cross sections for the emission of complex fragments requires suitable expressions for the level densities and the saddle point energies as a function of mass asymmetry and angular momentum. For all level densities the Fermi gas expression [Be 36, Bo 69] was used:

$$
\rho(U, J)=(2 J+1)\left(\frac{\Lambda^{2}}{2 I}\right)^{\frac{3}{2}} \frac{\sqrt{2}}{12} \frac{\exp (2 \sqrt{2 U})}{U^{2}},
$$

where $I$ is the moment of inertia of the system, $U$ is the thermal energy of the system, and a is the nuclear level density parameter, here $2=\mathrm{ACN} / 8.5 \mathrm{MeV}-1$. The saddle point energies, as a function of mass asymmetry and angular momentum, were calculated by Carjan and Alexander [Ca 88] using the Rotating Finite Range Model (Yukawa-plusexponential potential, plus a surface diffuseness term) [Si 86].

At each decay step all possible binary decays, from neutron and proton evaporation through symmetric fission, were considered. After each decay process, the heavy ( $\mathrm{Z} \geq 3$ ) secondary fragments were allowed to decay again until all the excitation energy was 
exhausted. Following the eilission of a heavy fragment, the remaining excitation energy was apportioned assuming equal temperatures in the two fragments. The angular momentum partition was calculated in the sticking limit. Thermal fluctuations in both the excitation energy division [Mo 81b] and the angular momentum partition [Mo 30, Sc 82] were incorporated.

Calculating the charge distributions requires the summation of the entrance channel L-waves as

$$
\sigma(Z)=\pi \times 2 \sum_{l=0}^{l-l_{\max }}(2 \ell+1) T_{l}(Z)
$$

where $T_{l}(Z)$ are the probabilities of the given decays proceeding at angular momentum $l$. This "sharp cut-ofr" approximation has been refined by introducing a diffuseness parameter to smear out $h_{\max }$ [Va 83].

At low energy ( $<20 \mathrm{MeV} / \mathrm{u}$ ) wh:se complete fusion reactions dominate, the statistical model calculations have been summed to the $h_{\max }$ that provides the best fit to the experimental data. The shapes of the calculated and measured $\sigma(\mathrm{Z})$ distributions can be compared, as can the $\mathrm{h}_{\text {max }}$ used in the calculation and those predicted by fusion models [Ba 74, Ba 77, Sw 82, Bj 82]. Fits that provide good agreement with both the shapes of the experimental cross section and with the predictions of $l_{\max }$ by the fusion models have been obtained [Ch 88b, Ch 89].

At larger energies the incomplete fusion process sets in. There are two problems associated with applying the statistical emission theory to incomplete fusion reactions. First, it is not apparent that the triangular \&-wave distribution used for somplete fusion reactions represents the spin distribution of the incomplete fusion products. Second, the theoretical cross sections are very dependent upon the choice of $\mathrm{J}_{\max }$, and there are no reliable incomplete fusion models froin which this quantity can be determined. 
The simplest assumption is to retain the triangular shape of the spin distribution. One then assumes that for every entrance channel $l$-wave the fractional angular momentum transfer is equal to the fractional linear momentum transfer. Equation (23) above is replaced by:

$$
\sigma(Z)=\pi \times 2 \sum_{\ell=0}^{L-J_{\max } / f_{P}}(2 \ell+1) T_{l}(Z)
$$

with $J=f_{P} \downarrow$, where $J$ is the spin transferred to the incomplete fusion product, and $f_{P}$ is the fractional linear momentum transfer.

Although the theoretical angular momentum distribution of the incomplete fusion products could also be determined from the geometrical - kinematic model, this determination would require simulating compound nucleus decay for a large number of systems of varying mass, charge, excitation energy, and angular momentum. While it is likely that there is a distribution of systems produced in these reactions, the moolel may not accurately predict this distribution. Therefore we have chosen to use the much simpler procedure described above to simulate the decay of the "average" incomplete fusion product. Similarly we have not incorporated any diffuseness into the compound nucleus spin distributions; this is a technical refinement to an aiready uncertain process.

The histograms in Figure III.25 show the statistical decay calculations for ${ }^{146} \mathrm{Nd}$ compound nuclei. Table III. 2 gives the excitation energies and the fractional linear momentum transfers from the zero spectator momentum model, and the $\mathrm{J}_{\max }$ of the triangular distributions that were used in the calculations. For these incomplete fusion reactions, $J_{\max }$ should be regarded as no more than a fitting parameter. The statistical uncertainties associated with the Monte Carlo nature of the calculations are approximately $5 \%$.

The uncertainties in the input parameters of the statistical code, specifically in the 
angular momentum distribution, do not allow for quantitative comparisons with the experimental data. However, the agreement within the range of the experimental data is very good at $50 \mathrm{MeV} / \mathrm{u}$. The shape of the distributior and the absolute magnitude of the cross sections can be explained within a factor of 2 using reasonable assumptions about the excitation energy and angular momentum distribution. This is strong evidence for the statistici nature of the process. There are no inexplicably large cross sections due to nonequilibrium processes or due to the onset of another reaction mechanism.

At the higher bombarding energies the calculated distributions are nearly flat, whereas the experimental distributions are U-shaped. In order to reproduce the cross sections at lower energy, it was necessary to subtract the anisotropic yield from the total yield for Z-values near the that of the target. A similar procedure was attempted for the $\mathbf{8 0}$ and $100 \mathrm{MeV} / \mathrm{u}$ data for the $\mathrm{Z}$-values with anisotropic angular distributions. While it was previously shown that the differential cross sections of these products do not consist of flat components plus exponential components as they do at leiver energy (see Figure III.17), the compound statistical model allows for different shapes of the angular distributions depending upon the angular momentum, the moment of inertia, and the temperature of the nucleus.

In the statistical model, the quantity $\frac{\mathrm{J}^{2}}{2 \mathrm{I}_{\text {eff }} \mathrm{T}}$ determines the angular distributions of the decay products. Here $\mathrm{J}$ is the nuclear spin, $\mathrm{T}$ the nucle $\mathrm{r}$ temperature, and $\mathrm{I}_{\text {eff }}$ is the effective moment of inertia of the conditional saddle point, $I_{\text {eff }}=\frac{1}{I_{\text {eff }}}=\frac{1}{I_{11}}-\frac{1}{I_{\perp}}$, where $I_{11}$ and $I_{\perp}$ are, respectively, the moments of inertia parallel and perpendicular to the nuclear symmetry axis [Mo 75]. When the above quantity is large, the $d \sigma / d \theta$ angular distributions are constant with $\theta$. On the other hand, when the quantity is small, the angular distributions are proportional to $\sin \theta$. Angular distributions intermediate between these two extremes are possible as long as they are symmetric about $90^{\circ}$ in the frame of the compound nucleus. In the spirit of the statistical model, the experimental cross sections 
have been decomposed into components forward and backward of $90^{\circ}$. The smaller of these two components (the component forward of $90^{\circ}$ for the lighter fragments and backward of $90^{\circ}$ for the heavier fragments) was assumed to result entirely from statistical emission. The "absolute" statistical cross sections were then determined by multiplying the smaller component by two. These cross section are shown as the open points in Figure II.25.

The cross sections for fragments with isotropic angular distributions (solid points) have been used to determine the $J_{\max }$ of the GEMINI calculations. The underprediction of the statistical cross sections for Z-values $\leq 15$ in figure III.25 may indicate that the anisotropic component extends forward of $90^{\circ}$ for these fragments. The slight overprediction of the cross sections for the largest Z-values may be a systematic error related to the extrapolations of the limited angular distributions (see Figure III.17). As at $50 \mathrm{MeV} / \mathrm{u}$, it is possible to reproduce the symmetric cross sections at both 80 and 100 $\mathrm{MeV} / \mathrm{u}$ with reasconable assumptions about the excitation energy and angular momentum of the equilibrated system (see Table III.2). From the $\mathrm{J}_{\max }$ values used to fit the complex fragment cross sections near symmetry the total compound nucleus cross sections can be inferred. These values are given in Table III.2. While the cross sections for statistical emission of complex fragment rise with increasing bombarding energy, the inferred total compound nucleus cross sections decrease.

Gross has used his statistical multifragmentation model to calculate the decay of a $146 \mathrm{Nd}$ nucleus with $284 \mathrm{MeV}$ of excitation energy [ $\mathrm{Gr} 88$ ], which is the system produced in the $50 \mathrm{MeV} / \mathrm{u}{ }^{139} \mathrm{La}+{ }^{12} \mathrm{C}$ reaction. This model calculates the probabilities for the simultaneous disassembly of a system into several fragments. An angular momentum window of $39 \leq \ell \leq 86$ was assumed for the incomplete fusion product, and the freeze-out radius was taken to be $2.05 \mathrm{~A}^{1 / 3}$. As discussed in the introduction, the freeze-out radius is a critical parameter in statistical multifragmentation models because the phase space for the different decay channels is calculated within a sphere of this radius. 
Figure III.26 shows the model predictions for the $Z_{1}-Z_{2}$ correlation (compare with III.1). The code predicts "multifragmentation" events with two complex fragments and several light charged particles, which is in agreement with the observed results.

Predictions for the absolute cross sections are shown in Figure III.27. The calculated cross section distribution is fairly flat near symmetry, as is the experimental distribution. While the absolute cross sections are somewhat smaller than those measured experimentally, Gross claims that increasing the freeze-out radius parameter from 2.05 , which is used for proton-induced reactions with small angular momentum, to 2.30 , which may be more appropriate for the systems of this work, brings the data and calculation more into line.

This multifragmentation calculation reproduces the experimental data as well as that of GEMINI, which is based on sequential binary emission. The data collected in the experiment are not able to distinguish incontrovertibly the nature of the emitted light particles (simultaneous or sequential); in fact, it may be impossible to make such a distinction for light particles emitted from the composite system. However, the agreement of the charged particle loss with that calculated from statistical emission (Figure III.21), along with the demonstrated continuity of the complex fragment emission process from lower energy, where the sequential emission of light particles is well-established, suggest that the sequential decay mechanism is more likely. 
Table III.1

$$
{ }^{139} \mathrm{La}+{ }^{12} \mathrm{C}
$$

50MeVu Experiment_Zero Spectator Momentum Geometrical-Kinematic

Y/ $\mathbf{Y}_{\text {beam }}$

"P $\mathrm{PTR}$ "

mass

$\mathrm{E}^{*}(\mathrm{MeV})$

$80 \mathrm{MeV} / \mathrm{H}$

$\mathrm{Y} / \mathrm{Y}_{\text {beam }}$

"P $\mathrm{TR}$ "

mass

$\mathrm{E}^{*}(\mathrm{MeV})$

$100 \mathrm{MeV} / \mathrm{u}$

$\mathrm{Y} / \mathrm{Y}_{\text {beam }}$

"P $P_{\text {IR" }}$

mass

$\mathrm{E}^{*}(\mathrm{MeV})$ $.956 \pm .01$

$\begin{array}{cc}0.52 \pm 0.13 & 0.55 \pm 0.14 \\ 145.6 \pm 1.6 & 145.1 \pm 1.6 \\ 280 \pm 70 & 300 \pm 80\end{array}$

$.952 \pm .007$

$0.57 \pm 0.09$

$146.4 \pm 1.1$

$500 \pm 70$

$$
\begin{gathered}
0.57 \pm 0.09 \\
146.5 \pm 1.1 \\
625 \pm 90
\end{gathered}
$$

$0.61 \pm 0.1$

$146.0 \pm 1.2$

$$
540 \pm 80
$$

$.952 \pm .007$

$0.62 \pm 0.1$

$146.2 \pm 1.2$

$690 \pm 110$ 


\section{Table III.2}

$$
{ }^{139} \mathrm{La}+{ }^{12} \mathrm{C}
$$

$\begin{array}{lcccc}\mathrm{E} / \mathrm{A} & \mathrm{E}^{*}(\mathrm{MeV}) & \mathrm{P}_{\mathrm{TR}} & \mathrm{I}_{\max }(\mathrm{S}) & \sigma_{\mathrm{CN}}(\text { bams }) \\ 14 & 151 & 1.00 & 55 & 1.206 \\ 18 & 188 & 1.00 & 58 & 1.041 \\ 50 & 280 & 0.52 & 60 & 0.744 \\ 80 & 500 & 0.57 & 61 & 0.434 \\ 100 & 625 & 0.57 & 05 & 0.392\end{array}$




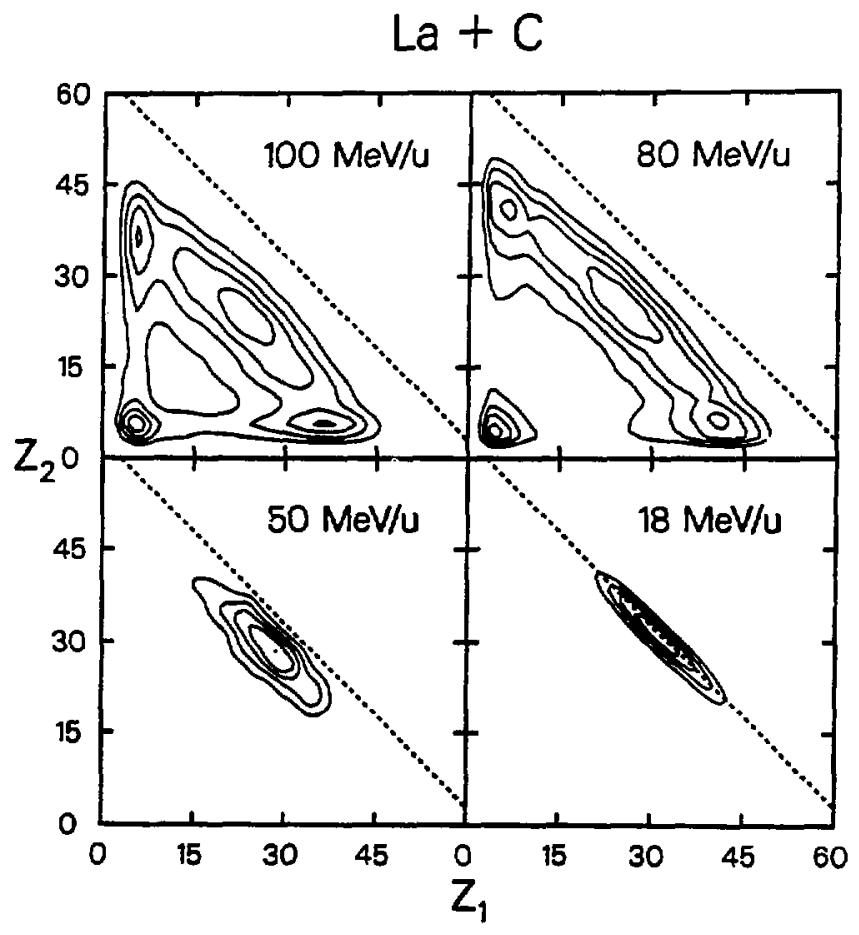

XBL $896-2524$

Figure III.1 Linear contour plots of 2 -fold $Z_{2}$ versus $Z_{1}$ coincidence events for the 18 , 50,80 , and $100 \mathrm{MeV} / \mathrm{u}{ }^{139} \mathrm{La}+{ }^{12} \mathrm{C}$ reactio 15 . The sets of four lines correspond to contours with relative ratios of 4:3:2:1. The oashed lines indicate the total charge of the projectile plus target (63). At $18 \mathrm{MeV} / \mathrm{u}$, the contours spread above this line due to the imperfect Z-resolution of the detectors and to the smoothing of the contour map. The distributions at 80 and $100 \mathrm{MeV} / \mathrm{u}$ have been reflected about the line $Z_{1}=Z_{2}$ to remove the bias due to the asymmetric detector configuration. 
$\mathrm{La}+\mathrm{C}$

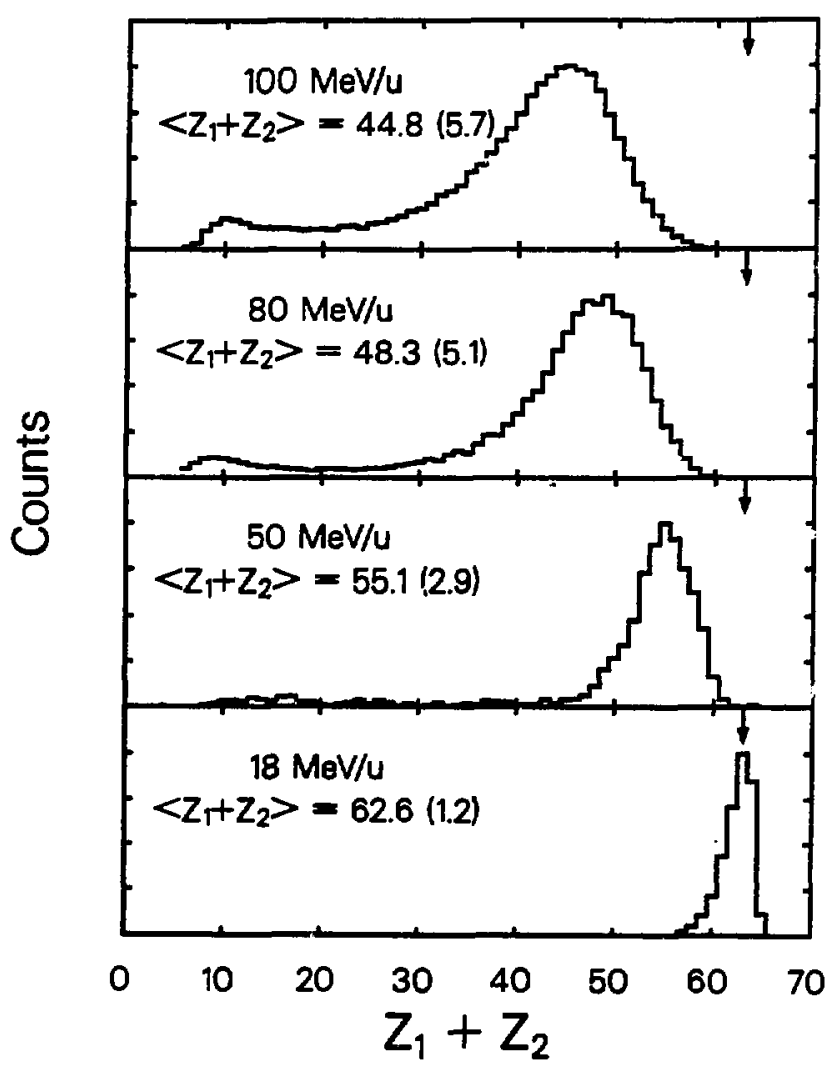

XEL 996-2513

Figure III.2 Distributions of the total detected charge $\left(\mathbf{Z}_{\mathbf{l}}+\mathbf{Z}_{2}\right)$ in 2-fold coincidence events for the $18,50,80$, and $100 \mathrm{MeV} / \mathrm{u}{ }^{139} \mathrm{La}+{ }^{12} \mathrm{C}$ reactions. The urrows indicate the total projectile and target charge (63). The centroid and (widith) of each distributions is shown. 


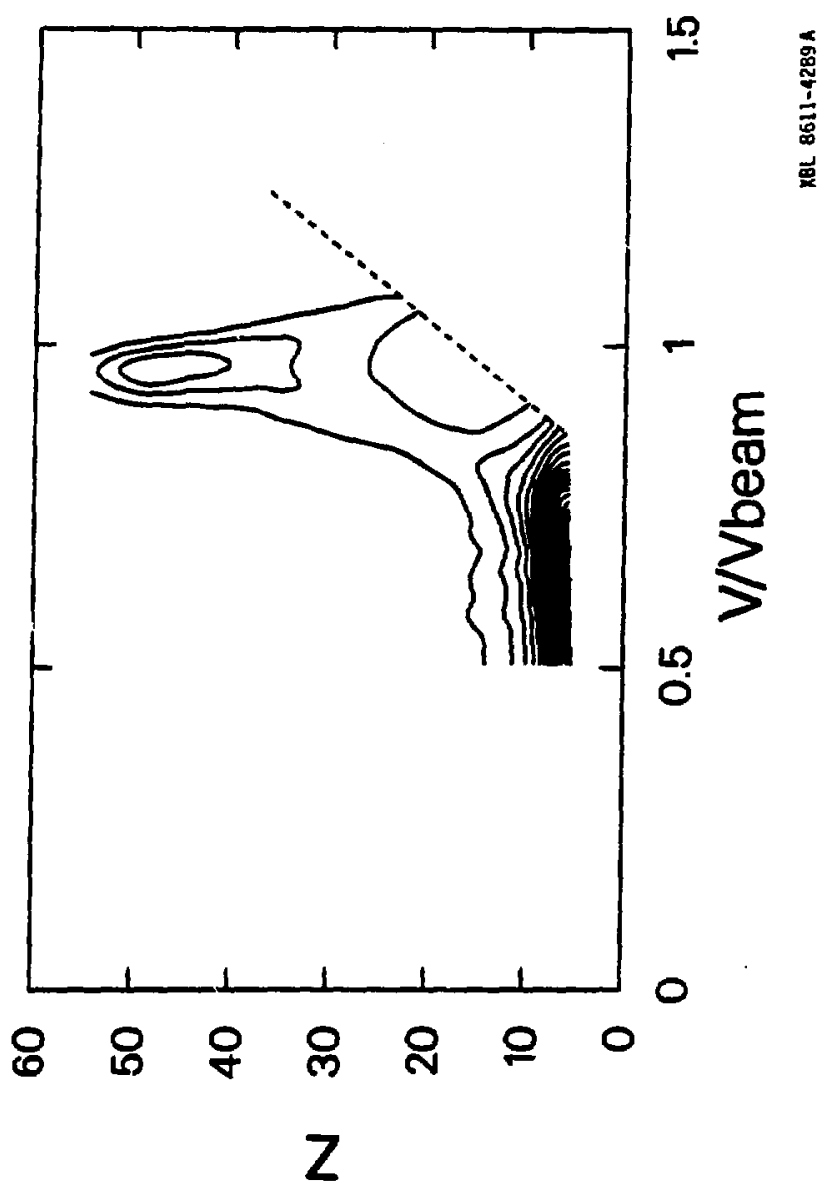

Figure III.3 Linear contours of the Galilean-invariant cross section, $\frac{d^{2} \sigma}{v^{2} d s i d v}$, in the Z velocity plane for the $50 \mathrm{MeV} / \mathrm{u} 139 \mathrm{La}+{ }^{12} \mathrm{C}$ reaction. There are a total of 37 contours with relative ratios of 1 to 37. The limits of detector acceptance were $3^{\circ}$ to $8^{\circ}$ in the lab The dashed line is the experimental threshold for particles which punched-through the $2 \mathrm{~mm}$ $\mathrm{Si}(\mathrm{Li})$ detectors. 


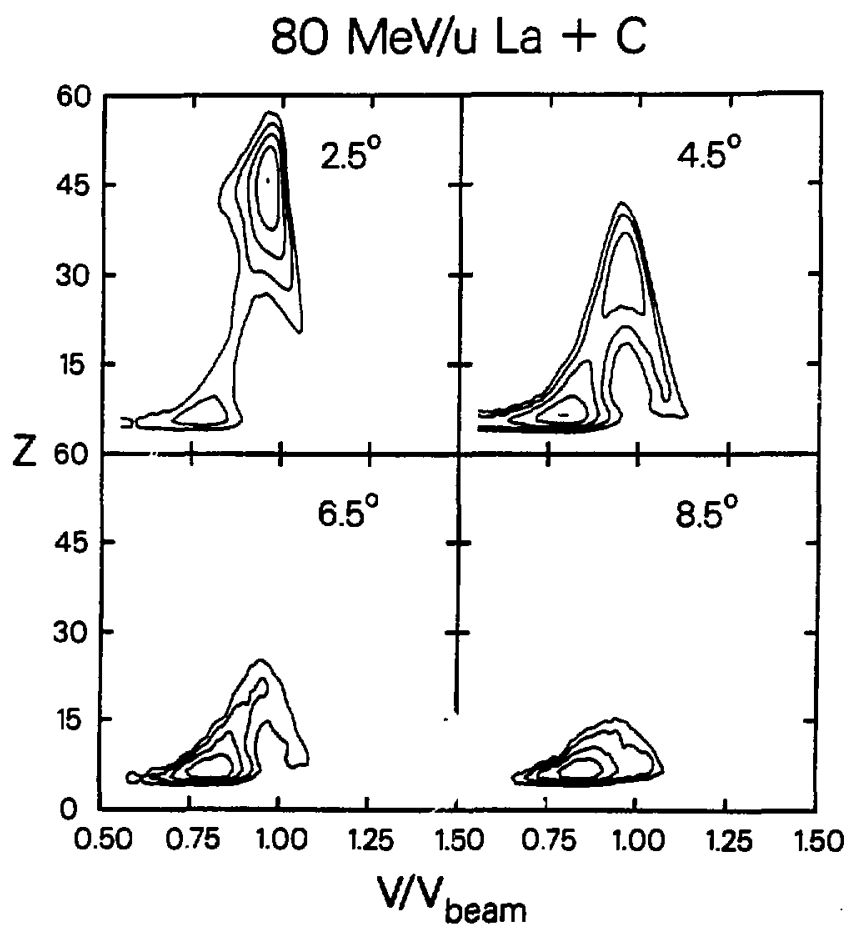

xaL $596-2911$

Figure III.4 Logarithmic contours of the Lorentz-invariant cross section, $\frac{d^{2} \sigma}{\gamma^{\prime} v^{2} d \Omega d v}$, in the $Z$ - velocity plane for the $80 \mathrm{MeV} / \mathrm{u}{ }^{139} \mathrm{La}+{ }^{12} \mathrm{C}$ reaction at laboratory angles of $2.5^{\circ}, 4.5^{*}, 6.5^{\circ}$, and $8.5^{\circ}$. Neighboring contours differ in value by approximately a factor of $S$ at $2.5^{\circ}$, a factor of 4 at $4.5^{\circ}$, a factor of 3 at $6.5^{\circ}$, and a factor of 3 at $8.5^{\circ}$. 


\section{$100 \mathrm{MeV} / \mathrm{u} \mathrm{La}+\mathrm{C}$}

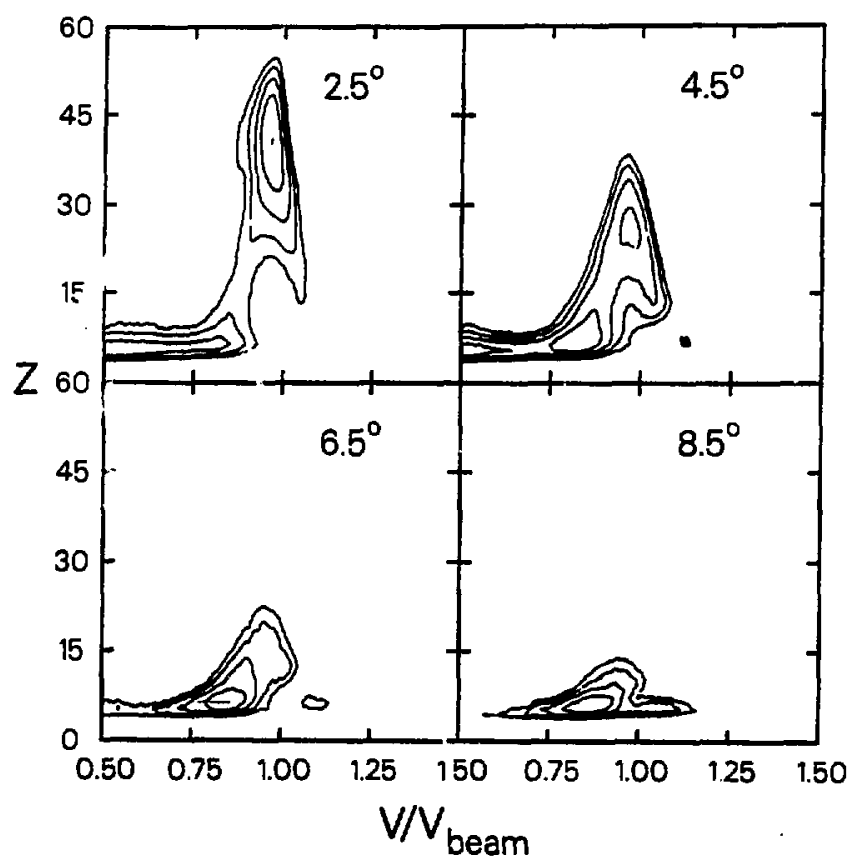

XEL $196-2512$

Figure III.5 Logarithmic contours of the Lorentz-invariant cross section, $\frac{d^{2} \sigma}{\gamma^{4} v^{2} d \Omega d v}$, in the $Z$ - velocity plane for the $100 \mathrm{MeV} / \mathrm{u}^{139} \mathrm{La}+{ }^{12} \mathrm{C}$ reaction at laboratory angles of $2.5^{\circ}, 4.5^{\circ}, 6.5^{\circ}$, and 8.5*. Neighboring contours differ in value by approximately a factor of 5.5 at $2.5^{\circ}$, a factor of $4.5^{\circ}$ at $4.5^{\circ}$, a factor of 3.5 at $6.5^{\circ}$, and a factor of 3.5 at $8.5^{\circ}$. 


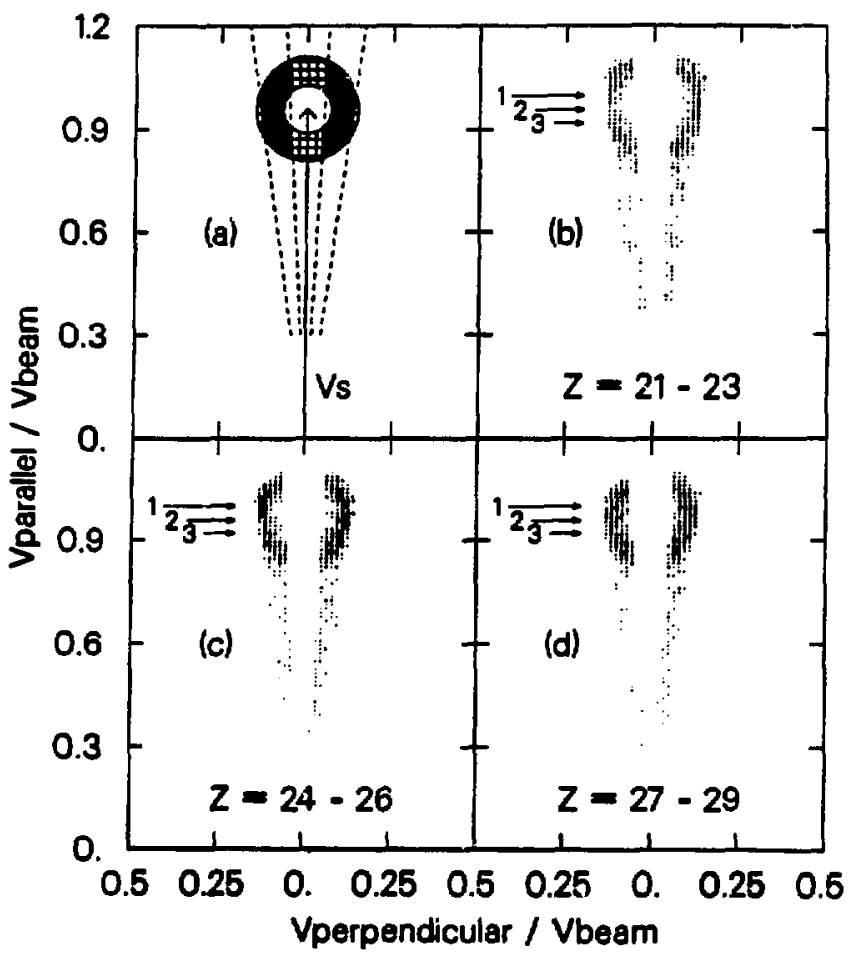

xec $611-4268$

Figure III.6 (a) Schematic representation of complex fragment emission in the $v_{\|}-v_{\perp}$ plune from a compound nucleus with a well-defined velocity, $V_{S}$. The geometric limits of detector acceptance are shown by the dashed lines, the solid area is the predicted experimental distribution. $(b, c, d)$ Linear density plots of $\frac{\partial^{2} \sigma}{\partial v_{11} \partial v_{\perp}}$ in the $v_{\| 1}-v_{\perp}$ plane for several $Z$ bins in the $50 \mathrm{MeV} / \mathrm{u}{ }^{139} \mathrm{Lz}+{ }^{12} \mathrm{C}$ reaction. The size of the points indicates the density of the distribution. Arrows 1,2 , and 3 denote the beam velocity, the extracted cource velocity, and the velocity for complete fusion, respectively. 


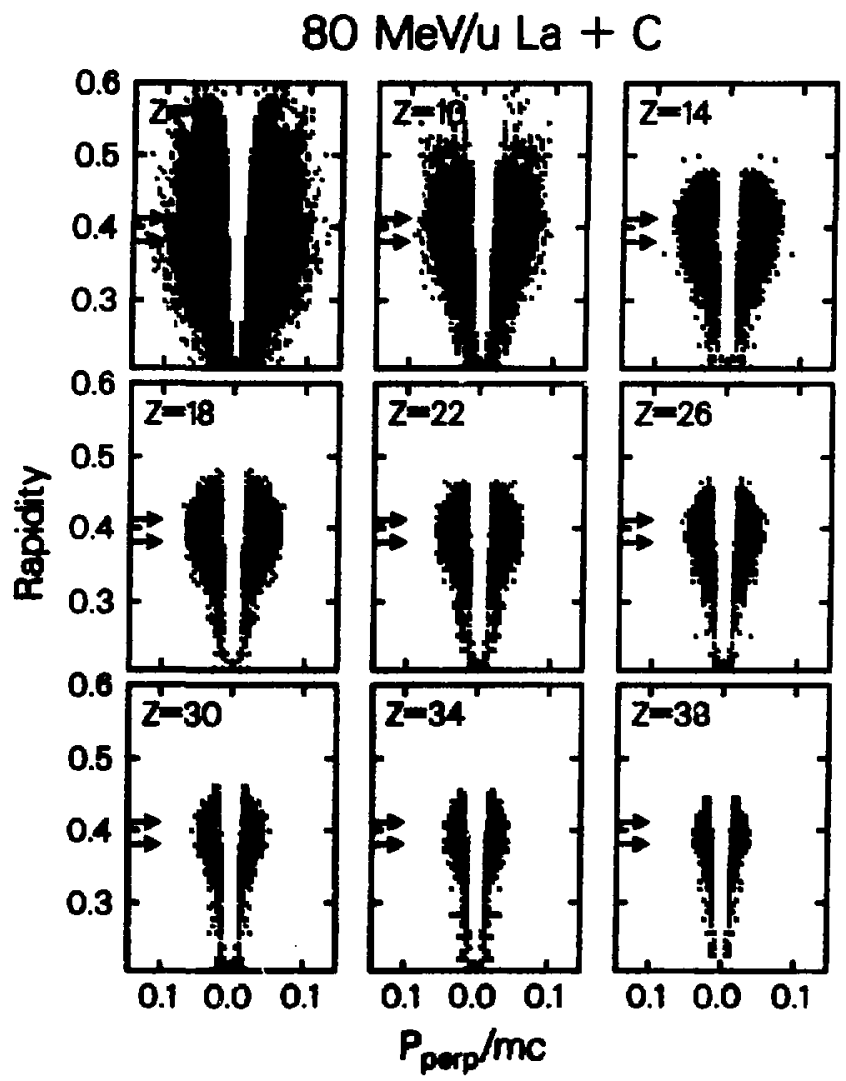

Figure III.7 Linear density plot of $\frac{\partial^{2} \sigma}{\partial \mathrm{Y} \partial\left(\mathbf{P}_{\perp} / \mathrm{mc}\right)}$ in the rapidity - perpendicular momentum plane for representative Z-values between 6 and 38 in the $80 \mathrm{MeV} / \mathrm{u}^{139} \mathrm{La}+$ ${ }^{12} \mathrm{C}$ reaction. The upper arrow in each subplot corresponds to the beam rapidity and the lower arrow to the rapidity of the center-of-mass. Red indicates the regions of highest intensity. 


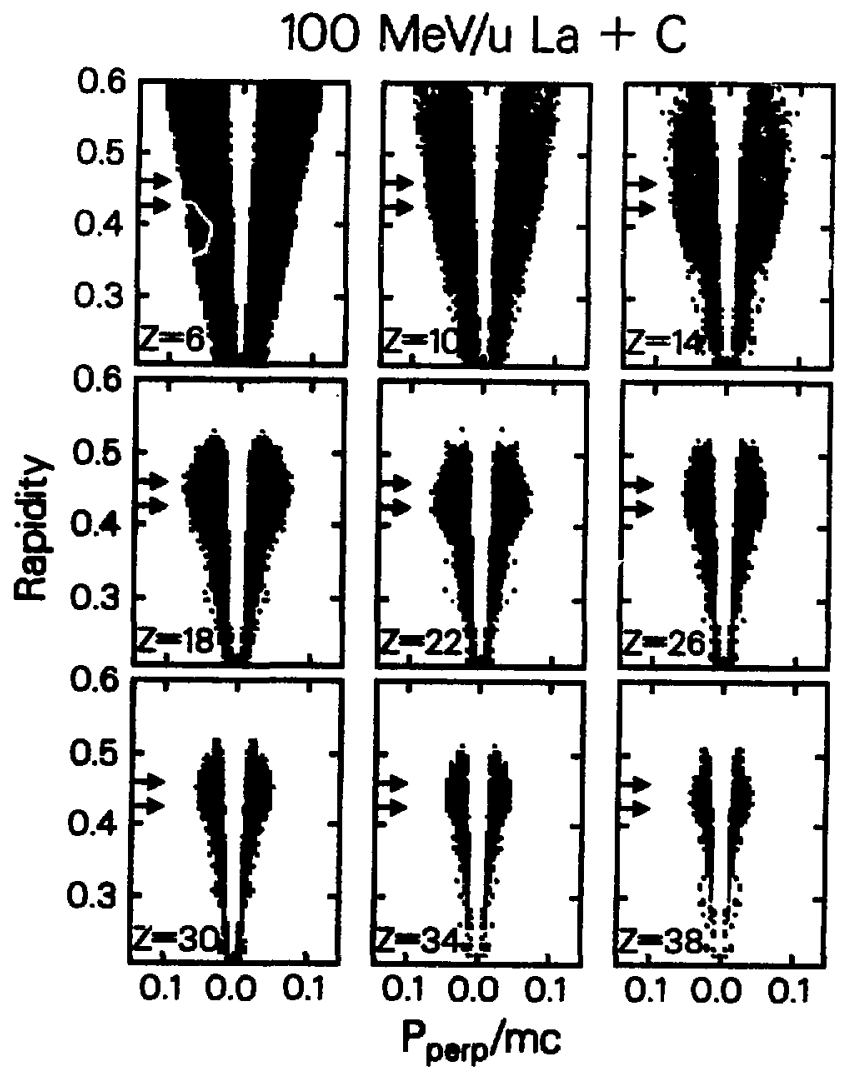

Figure III.8 Linear density plot of $\frac{\partial^{2} \sigma}{\partial Y \partial\left(P_{\perp} / \mathrm{mc}\right)}$ in the rapidity - perpendicular momentum plane for representative $\mathrm{Z}$-values between 6 and 38 in the $100 \mathrm{MeV} / \mathrm{u}$ ${ }^{139} \mathrm{La}+{ }^{12} \mathrm{C}$ reaction. The upper arrow in each subplot corresponds to the beam rapidity and the lower arrow to the rapidity of the center-of-mass. Rec indicates the regions of highest intensity. 


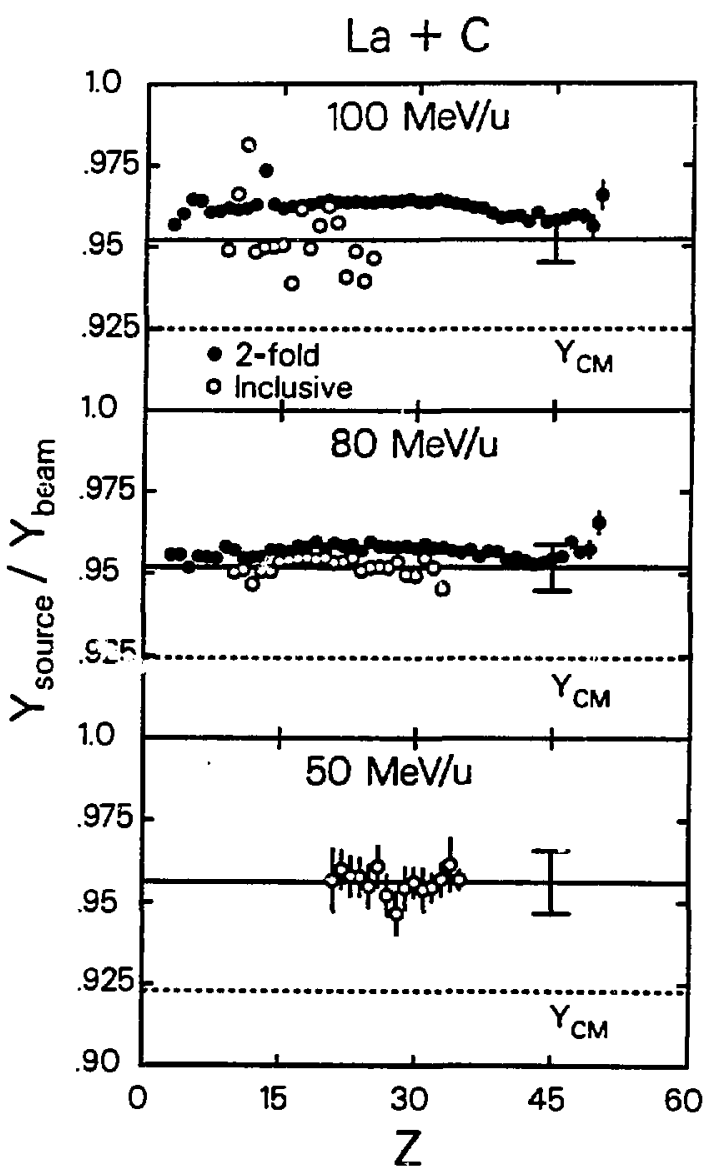

XBL $196-2495$

Figure III.9 Ratios of the source rapidity to the beam rapidity as a funcrion of Z-value extracted from the inclusive and 2-fold coincidence events for the 50,80 , and $100 \mathrm{MeV} / \mathrm{u}$ ${ }^{139} \mathrm{La}+{ }^{12} \mathrm{C}$ reactions. The bars on each point at $50 \mathrm{MeV} / \mathrm{u}$ are the statistical errors; at the higher energies the statistical errors are smaller than the data points. The solid lines correspond to the mean source rapidity as determined from the inclusive data, the large error bars indicate the possible systematic errors associated with the energy calibrations and the mass parametrization. The dashed lines are the rapidities which correspond to complete fusion. 


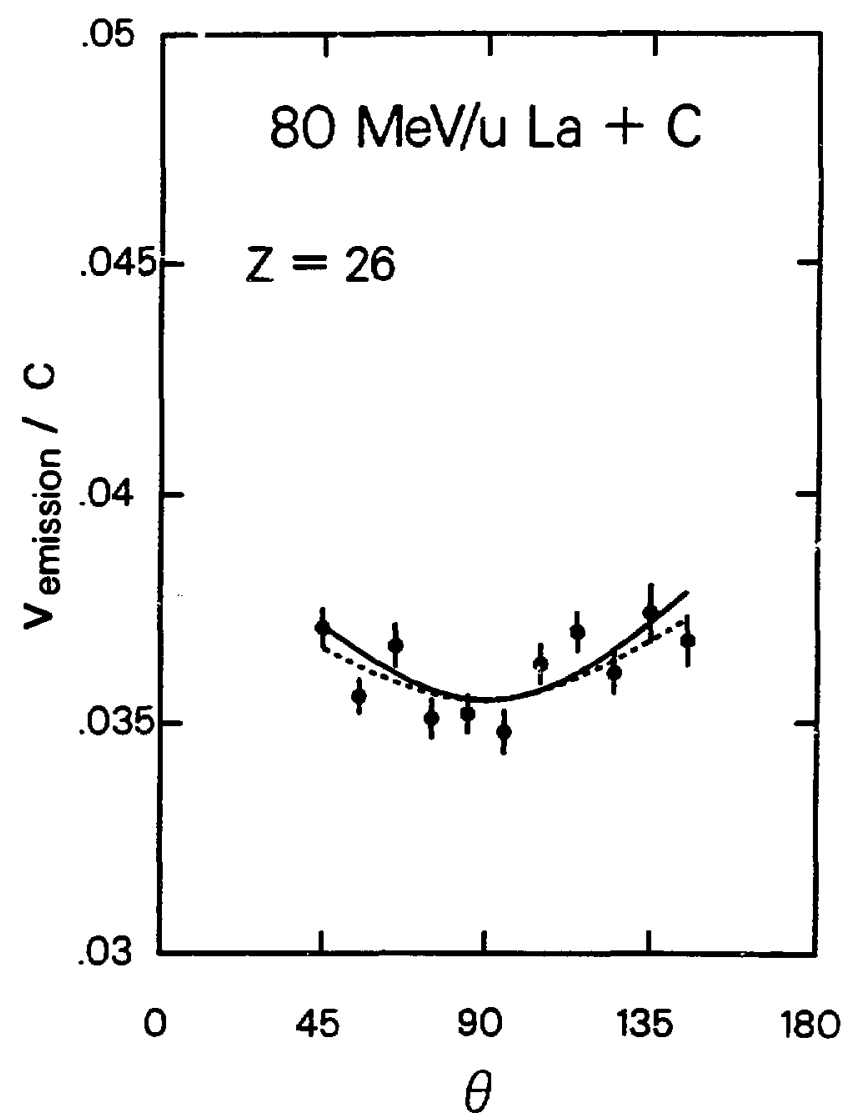

XBL 896-2497

Figure III.10 The emission velocity as a function of emission angle for $\mathrm{Z}=26$ fragments in the $80 \mathrm{MeV} / \mathrm{u}{ }^{139} \mathrm{La}+{ }^{12} \mathrm{C}$ reaction. The solid points are the experimental data with error bars. The dashed line is a least squares fit of the data points to an elliptical distribution. The solid line is the prediction of a Monte Carlo program that assumes emission with a well-defired velocity from a range of sources with the measured rapidity distribution (see Figure III.20). 


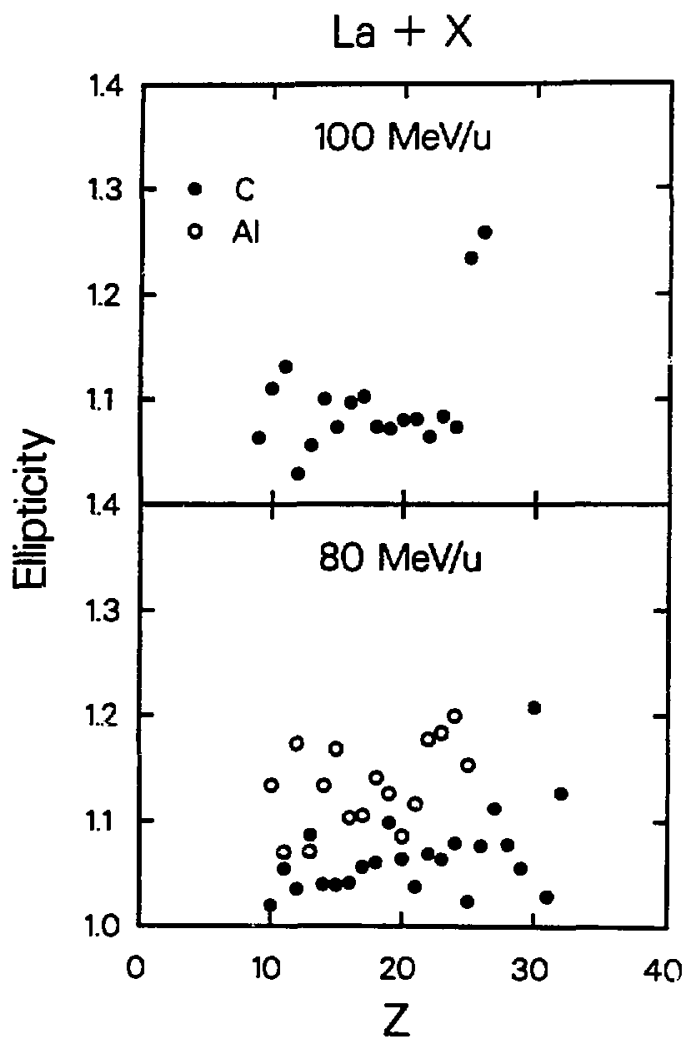

XoL $896-2498$ A

Figure III.11 The ratio of the major to minor axes of the elliptical fits to the $80 \mathrm{MeV} / \mathrm{u}$ ${ }^{139} \mathrm{La}+{ }^{12} \mathrm{C},{ }^{27} \mathrm{Al}$ and the $100 \mathrm{MeV} / \mathrm{u}{ }^{139} \mathrm{La}+{ }^{12} \mathrm{C} \frac{\partial^{2} \sigma}{\partial \mathrm{Y} \partial\left(\mathrm{P}_{\perp} / \mathrm{mc}\right)}$ distributions. In all cases the major axes are parallel to the beam direction. 
$\mathrm{La}+\mathrm{C}$

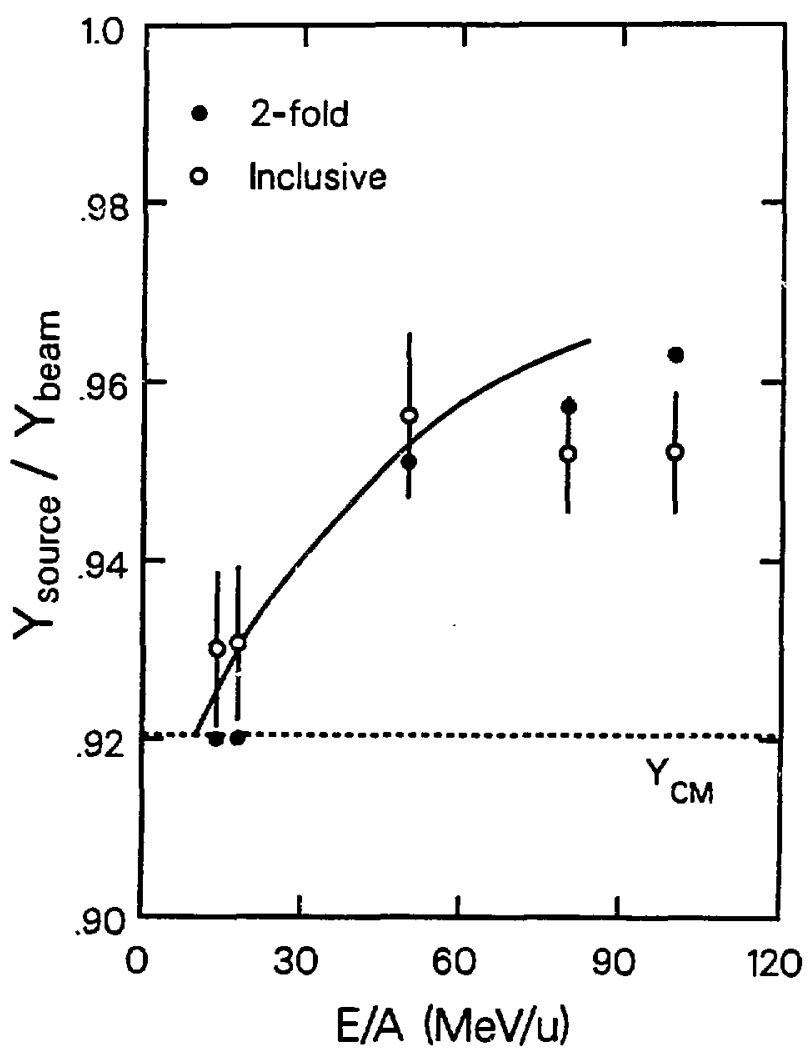

XBL $896-2499$

Figure III.12 The average ratios of the cource rapidity to the beam rapidity for ${ }^{139} \mathrm{La}+$ ${ }^{12} \mathrm{C}$ reactions as a function of bombarding energy as determined from inclusive and 2-fold complex fragment events. All of the statistical errors are smaller than the data points. The bars on the inclusive points indicate the possible systematic errors from the energy calibrations and the mass parametrization. The solid line corresponds to the experimental momentum transfer systematics [Vi $82, \mathrm{Ga} 82$, Ch 83b, Mo 84, St 85]. 


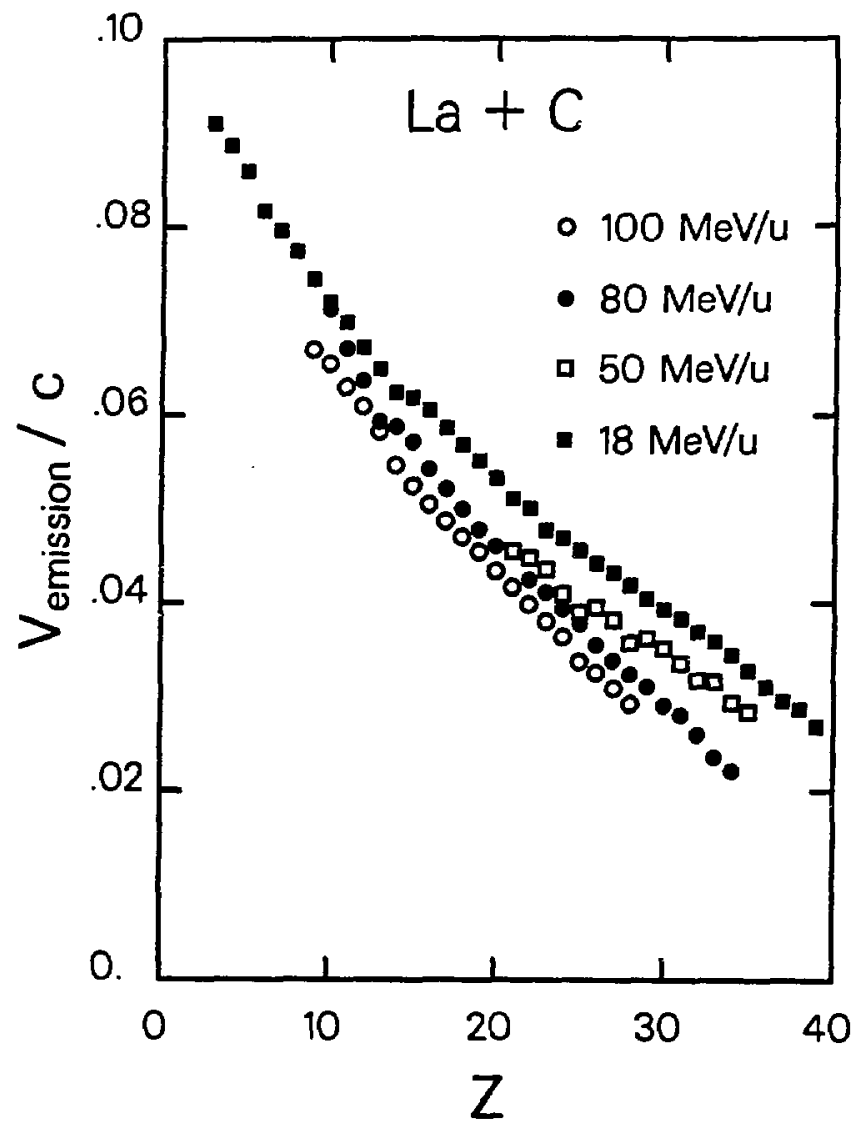

XBL $896-2500$

Figure III.13 The emission velocities as a function of Z-value extracted from the inclusive data in the $18,50,80$, and $100 \mathrm{MeV} / \mathrm{u}{ }^{139} \mathrm{La}+{ }^{12} \mathrm{C}$ reactions. In all cases the statistical errors are smaller than the data points. 


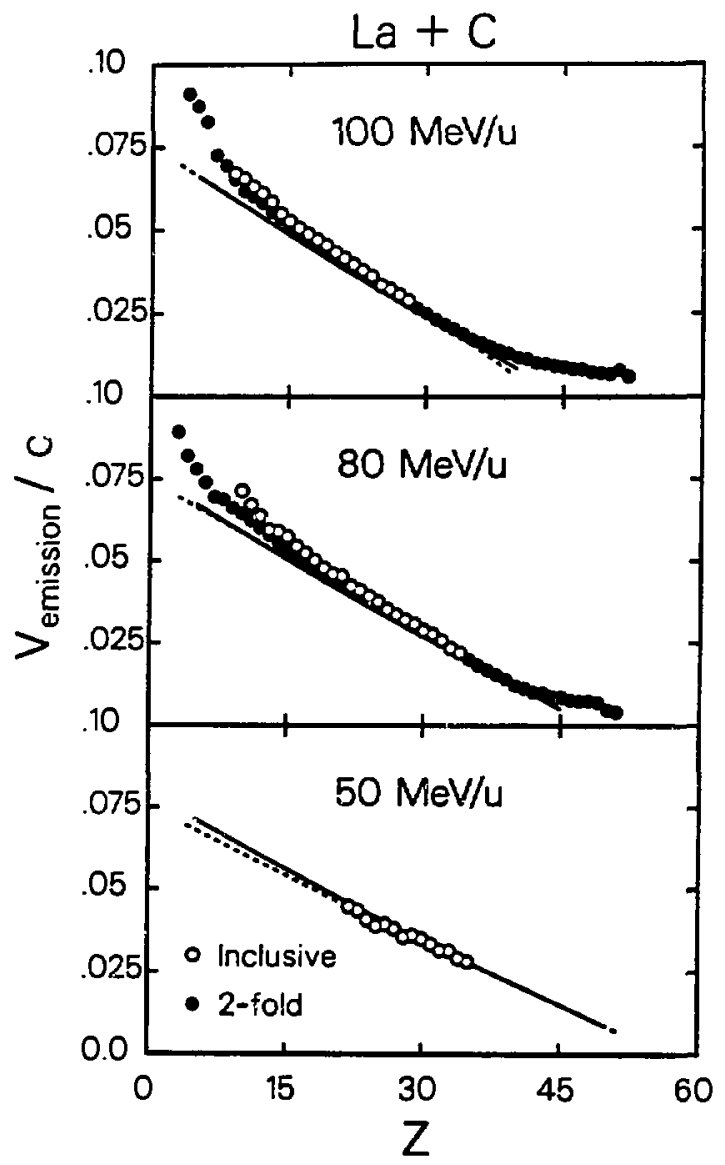

XEL 896-2501

Figure III.14 The emission velocities as a function of Z-value from inclusive and 2-fold coincidence events for the 50,80 , and $100 \mathrm{MeV} / \mathrm{u}^{139} \mathrm{La}+{ }^{12} \mathrm{C}$ reactions. In all cases the statistical errors are smaller than the data points. The lines are predictions of the expected emission velocities from the Viola fission fragment kinetic energy systematics [Vi 85], which were generalized to include decays at all charge asymmetries. The dashed line shows the predicted emission velocities assuming that the binary decay precedes all of the light particle emission. The solid line shows the predicted velocities assuming that the binary decay follows all of the light particle emission. 


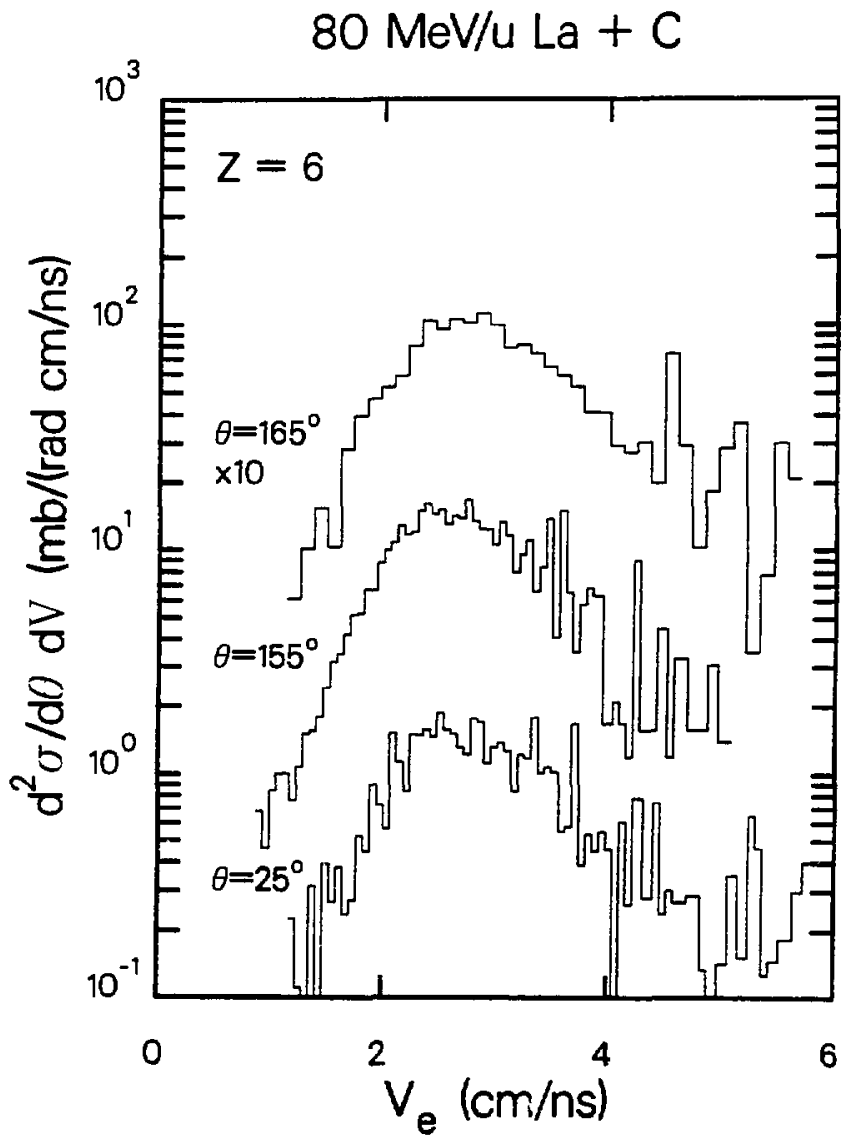

X.L $896-2502$

Figure III.15 Emission velocity distributions for $Z \approx 6$ fragments in the $80 \mathrm{MeV} / \mathrm{u} 130 \mathrm{La}$ $+{ }^{12} \mathrm{C}$ reaction at angles of $25^{\circ}, 155^{\circ}$, and $165^{\circ}$ in the source frame. The distribution at $165^{\circ}$ has been multiplied by a factor of 10 . 


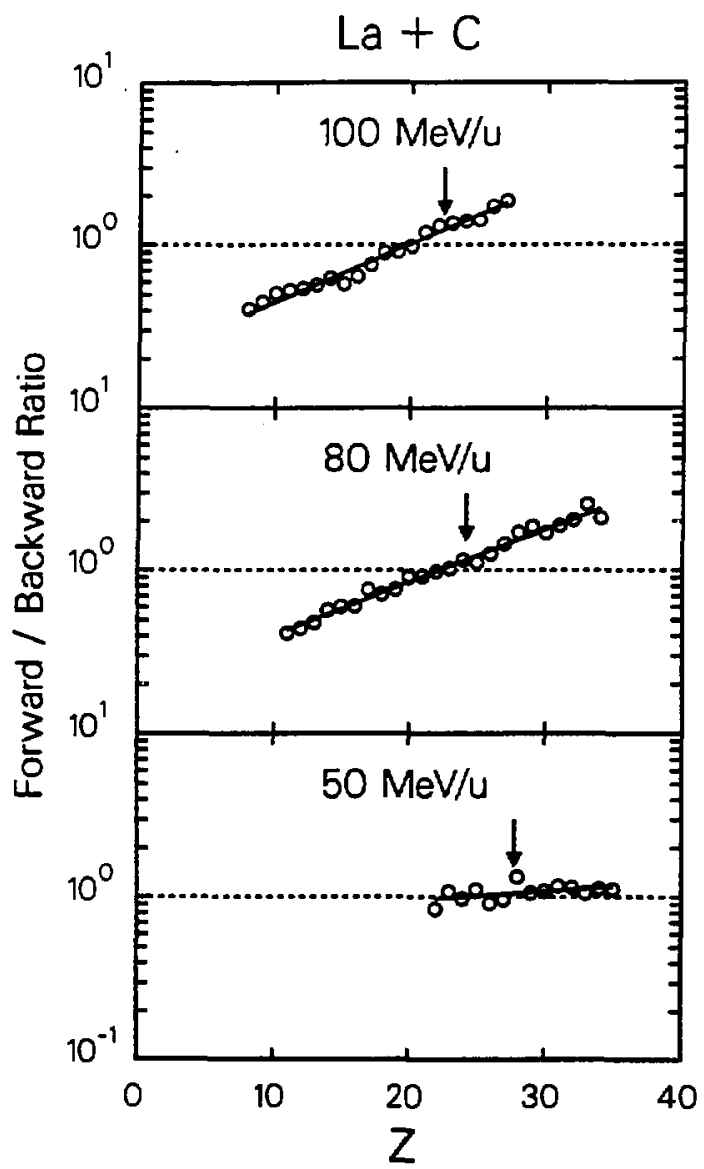

XBL $896-2503$

Figure III.16 The ratio of fragments emitted forward of $90^{\circ}$ to those emitted backward of $90^{\circ}$ (in the source frame) as a function of fragment Z-value for the 50,80 , and $100 \mathrm{MeV} / \mathrm{u}$ ${ }^{139} \mathrm{La}+{ }^{12} \mathrm{C}$ reactions. The solid lines are linear least squares fits to the $\log$ of the ratios. The arrows indicate the average $Z$-values corresponding to symmetric decay as determined by the coincidence data $\left(Z_{\text {sym }}=\frac{1}{2}\left(\overline{\left.Z_{1}+Z_{2}\right)}\right)\right.$. The dashed line $(=1)$ corresponds to forward/backward angular symmetry. 


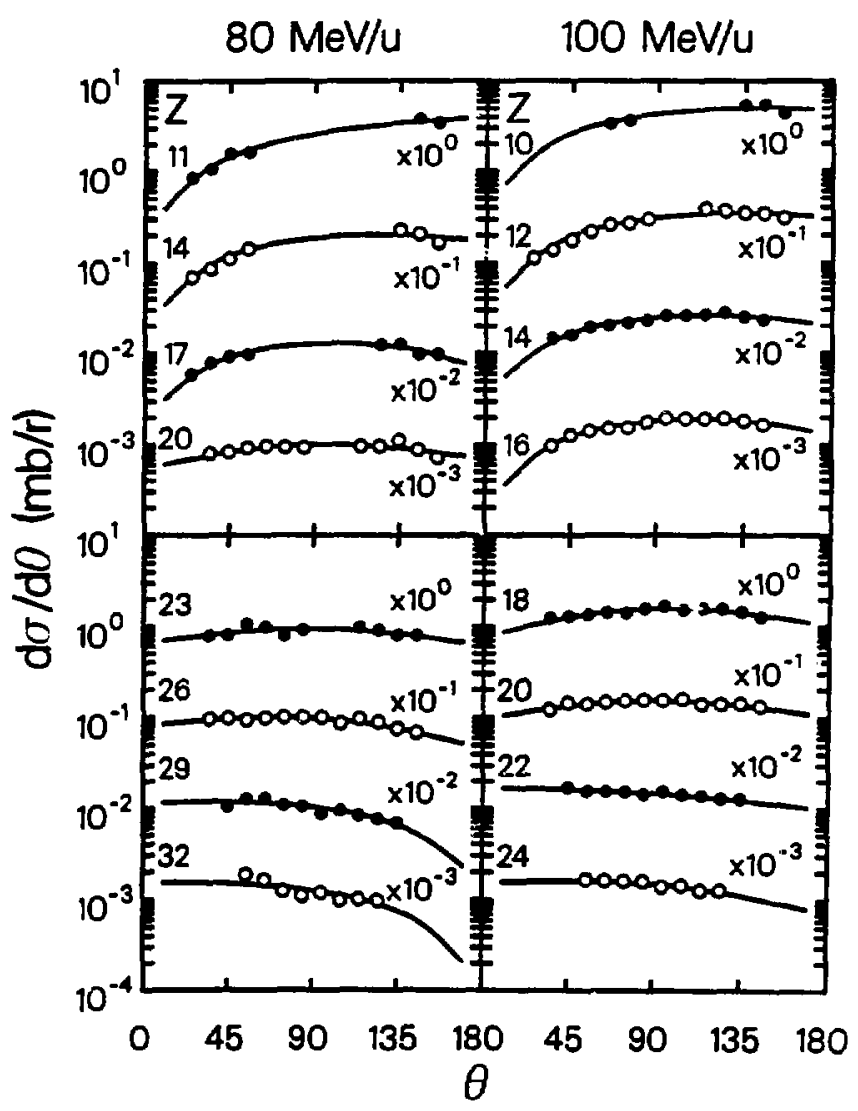

XaL $896-2504$

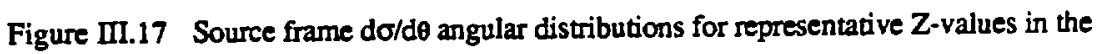
80 and $100 \mathrm{MeV} / \mathrm{u}{ }^{139} \mathrm{La}+{ }^{12} \mathrm{C}$ reactions. The $\mathrm{Z}$-value and normalization are indicated for each set of data. The solid lines show the fits to the distributions used to extract the absolute cross sections. At emission angles near $90^{\circ}$, the lighter complex fragments are emitted to larger laboratory angles than covered by the detector array, so the differential cross sections could not be measured. 


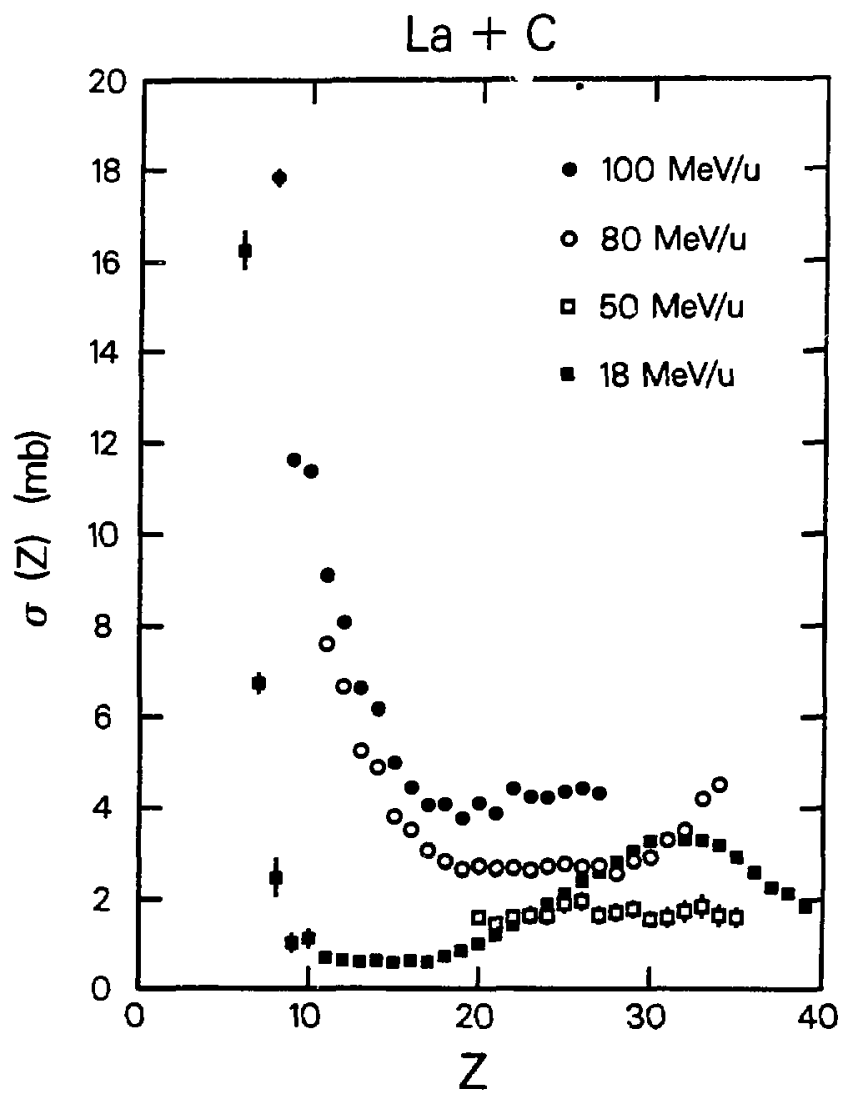

XEL $096-2505$

Figure III.18 Angle-integrated cross sections of products in the 18,50,80, and 100 $\mathrm{MeV} / \mathrm{u}{ }^{139} \mathrm{La}+{ }^{12} \mathrm{C}$ reactions. The bars on some of the points are the statistical errors, where bars do not appear the errors are smaller than the size of the data points. The possible systematic errors associated with the absolute beam normalization, target thickness, and the integration procedure are $20 \%$ at $18 \mathrm{MeV} / \mathrm{u}, 50 \%$ at $50 \mathrm{MeV} / \mathrm{u}, 20 \%$ at $80 \mathrm{MeV} / \mathrm{u}$ and $20 \%$ at $100 \mathrm{MeV} / \mathrm{u}$. 
$\mathrm{La}+\mathrm{C}$

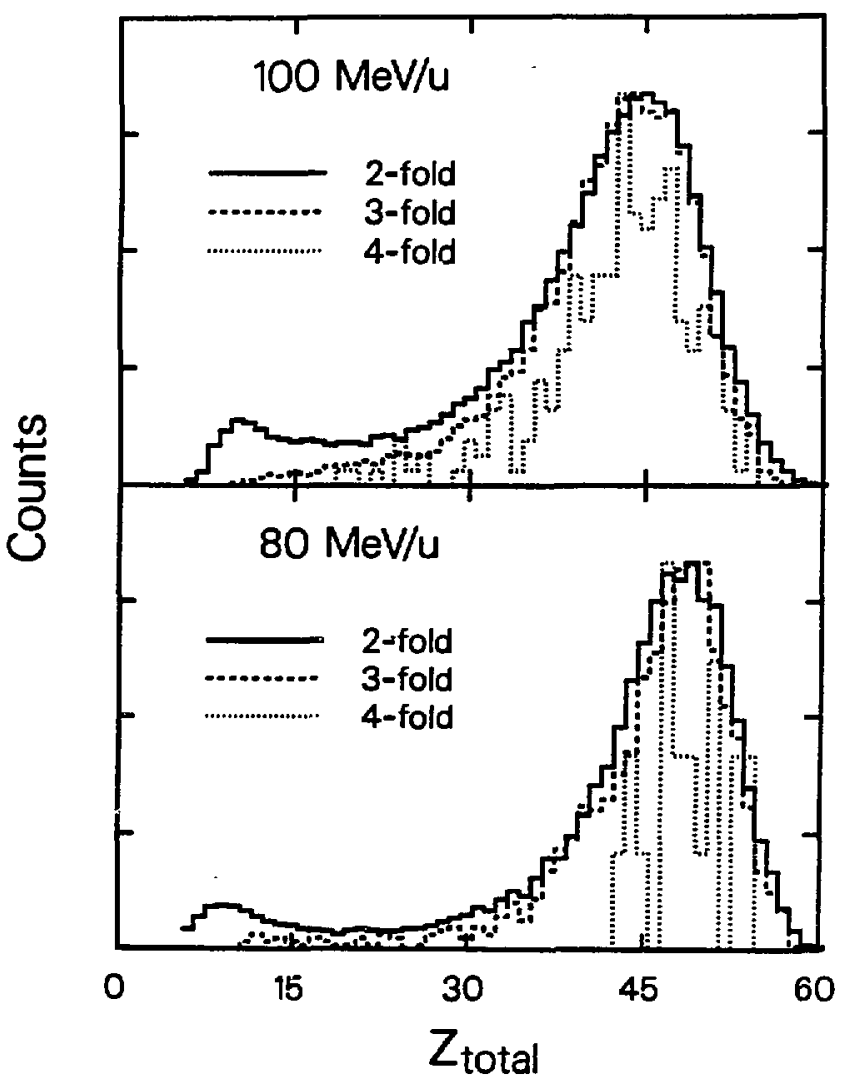

X2L 896-2506

Figure III.19 Distributions of $Z_{\text {Toeal }}\left(Z_{1}+Z_{2}\right)$ for 2-fold, 3-fold, and 4-fold complex fragment $(Z>2)$ events in the 80 and $100 \mathrm{MeV} / \mathrm{u}{ }^{139} \mathrm{La}+{ }^{12} \mathrm{C}$ reactions. The maxima of the distributions have been normalized to each other. 


\section{$\mathrm{La}+\mathrm{C}$}

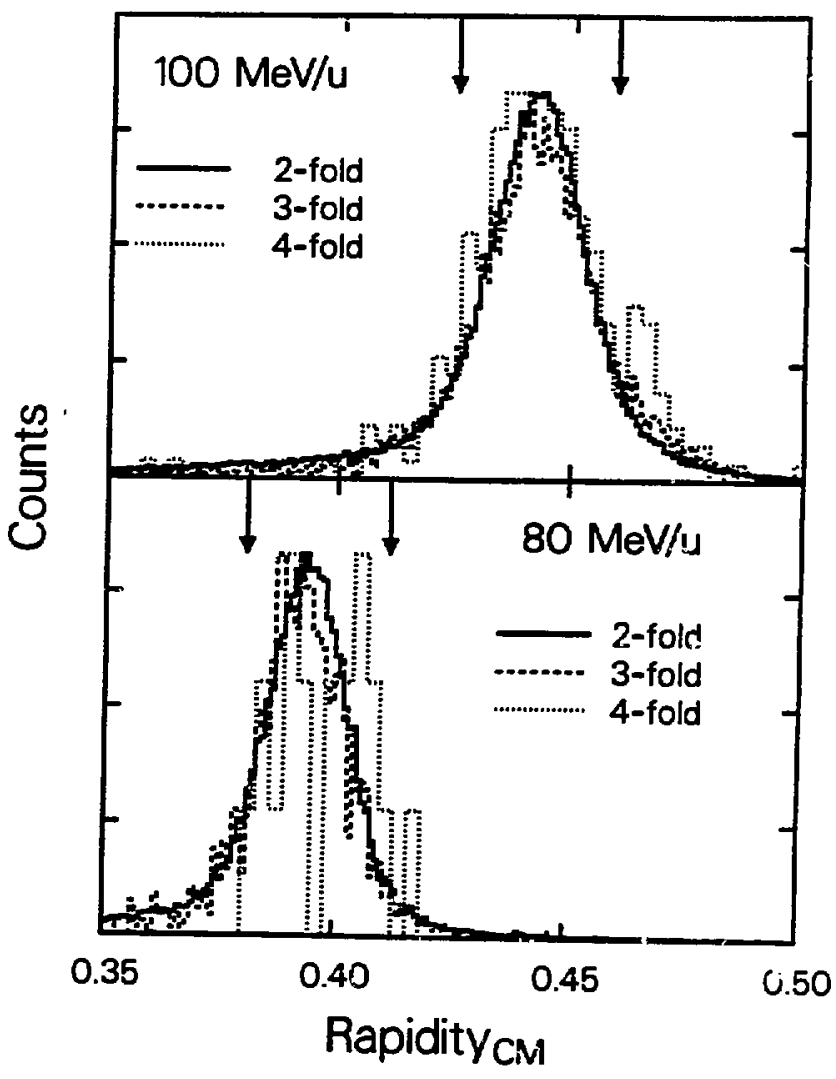

Y.L 896-2507

Figure III.20 Distributions of the center-of-mass rapidity for 2-fold, 3-fold, and 4-fold complex fragment $(Z>2)$ events in the 80 and $100 \mathrm{MeV} / \mathrm{u}{ }^{139} / \mathrm{a}+{ }^{12} \mathrm{C}$ reactions. The maxima of the distributions have been normalized to each other. The arrow at iarger rapidity in each subplot indicates the beam rapidity. The arrow at smaller rapidity indicates the center-of-mass rapidity of the entrince chinnel. 


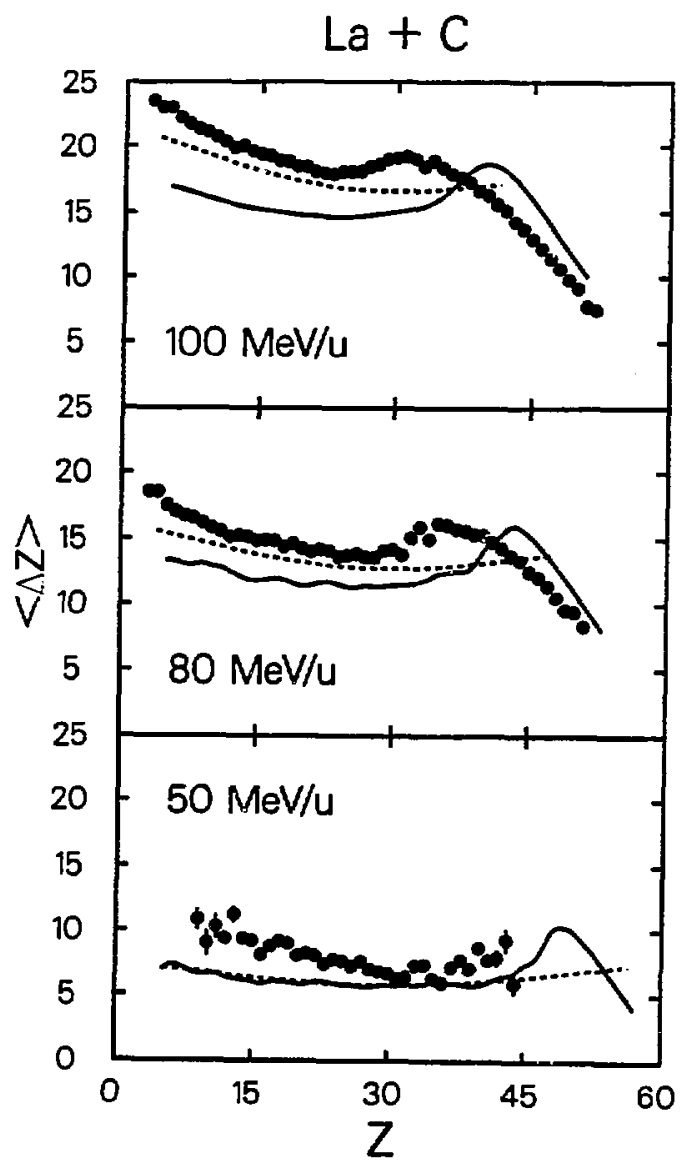

XBL 896-2509

Figure III.2i The average charge loss in coincidence events $\left[\left(Z_{\text {Projectile }}+Z_{\text {Target }}\right)-\left(Z_{1}+\right.\right.$ $\left.Z_{2}\right)$ ] as a function of the $Z$-value of ore of the coincident fragments in the 50,80 , and 100 $\mathrm{MeV} / 4{ }^{139} \mathrm{La}+{ }^{12} \mathrm{C}$ reactions. The solid points are experimental points. The solid line is the prediction from a statistical decay code (GEMINI) incorporating both complex fragment emission and light particle evaporation. The dashed line is the prediction from the statistical evaporation code PACE [Ga 80], assuming that the binary decay occun priur to the light particle evaporation. 


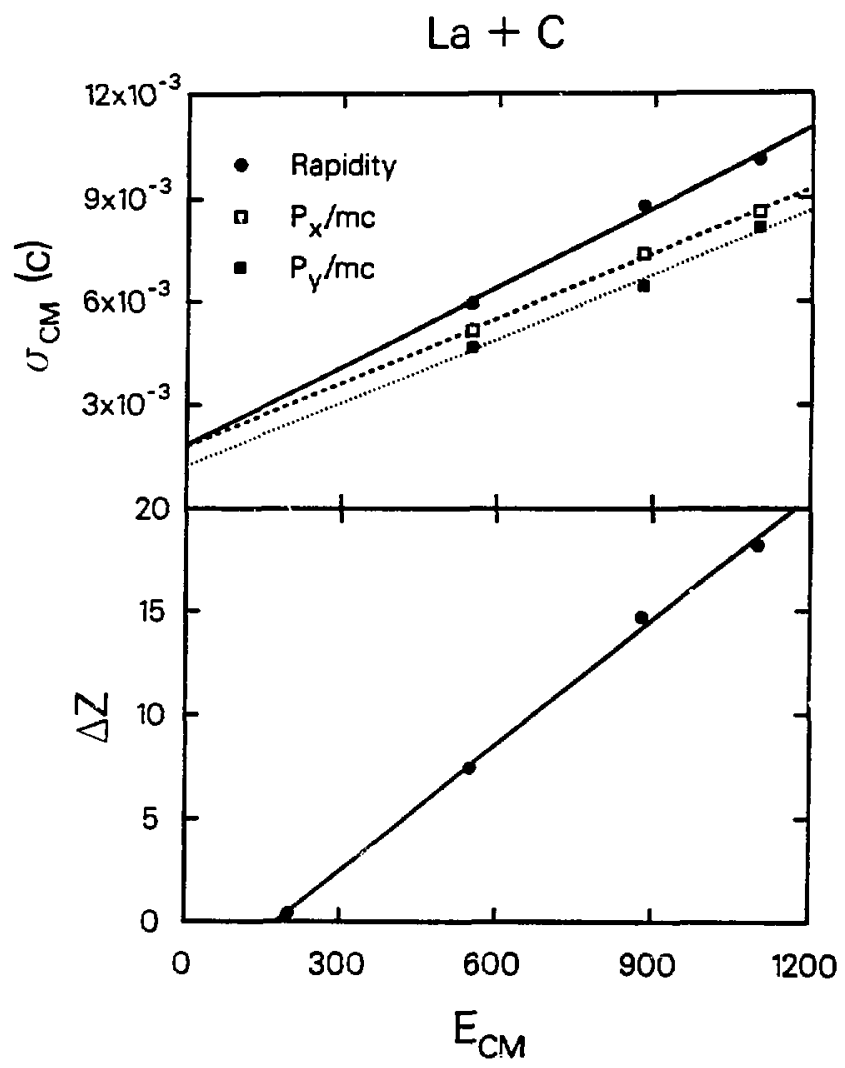

xp2 $996-2510$

Figure III.22 The upper part of the Figure shows the widths of the rapidity and momentum distributions as a function of the center-of-mass energy for ${ }^{139} \mathrm{La}+{ }^{12} \mathrm{C}$ reactions. The lower part of the Figure shows the average charge loss from coincidence events $\left[\left(Z_{\text {Projectile }}+Z_{\text {Target }}\right)-\overline{\left(Z_{1}+Z_{2}\right)}\right]$ as a function of the center-of-mass energy in the ${ }^{139} \mathrm{La}+{ }^{12} \mathrm{C}$ system. The solid lines in both parts of the Figure are linear least-squares fits to the data points. 


\section{$50 \mathrm{MeV} / \mathrm{u} \mathrm{La}+\mathrm{C}$}

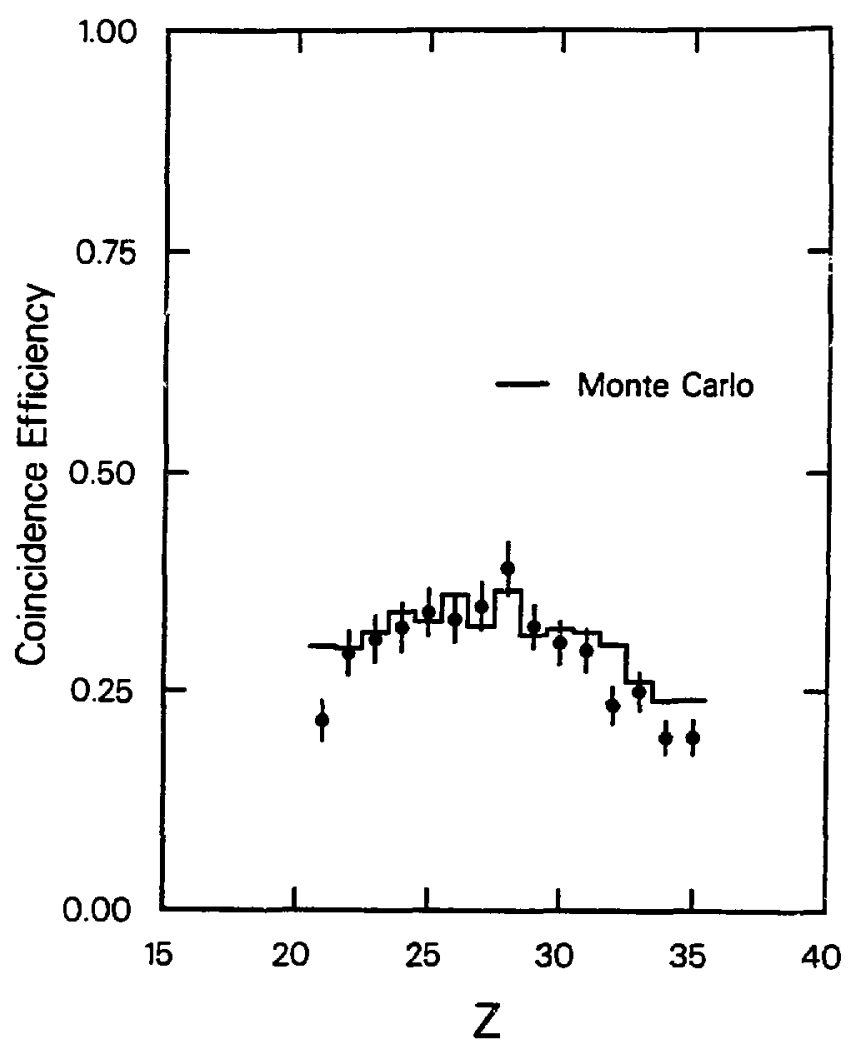

XaL $\$ 96-2508$

Figure III.23 The coincidence efficiency (ratio of coincidence events to inclusive events) as a function of Z-value for the $50 \mathrm{MeV} / \mathrm{u}{ }^{139} \mathrm{La}+{ }^{12} \mathrm{C}$ reaction. The solid points with error bars are the experimental data. The histogram is a Monte Carlo simulation of the coincidence efficiency assuming binary decay and incorporating the experimental velocity distributions, the detector geometry, and the beam spot size. 


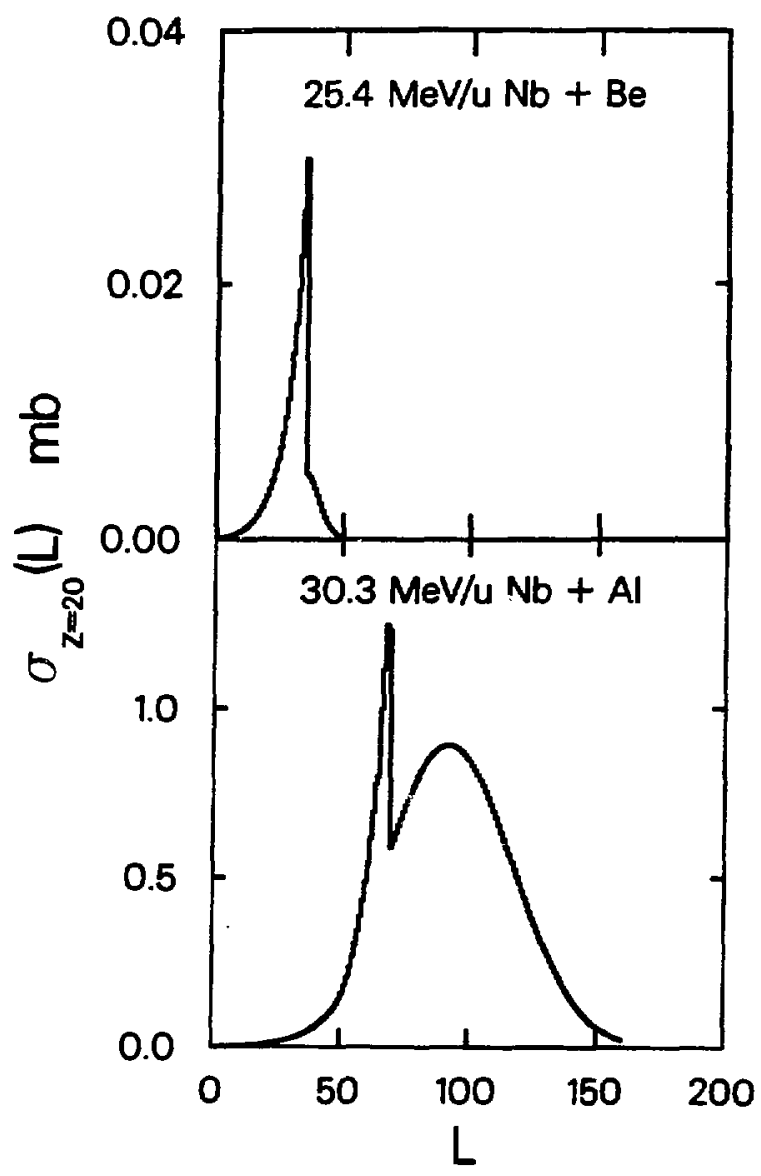

X8L 873-1335

Figure III.24 Calculated cross sections for $Z=20$ fragments as a function of the entrance channel angular momentum in the $25.4 \mathrm{MeV} / \mathrm{u}{ }^{93} \mathrm{Nb}+{ }^{9} \mathrm{Be}$ and $30.3 \mathrm{MeV} / \mathrm{u}{ }^{93} \mathrm{Nb}+{ }^{27} \mathrm{Al}$ reactions. The input parameters for the statistical decay model were determined using the incomplete fusion model described in Appendix II. 
104

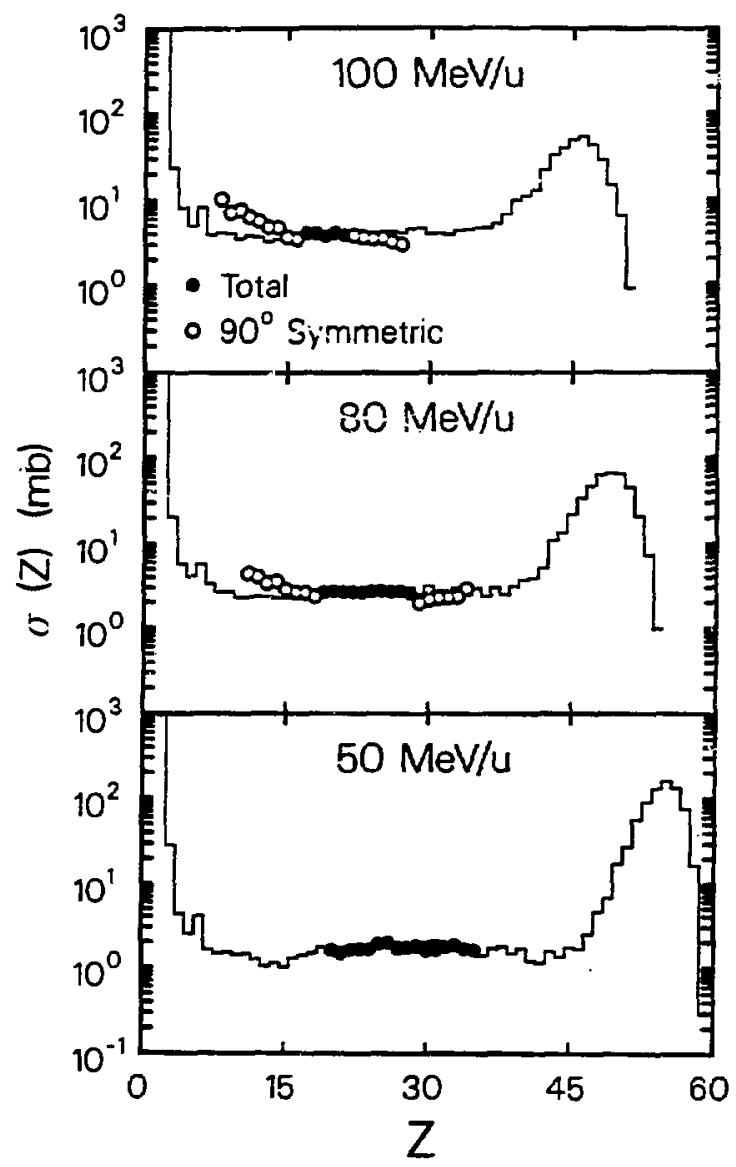

XBL $896-2494$

Figure III.25 Experimental complex fragment cross sections compared to predictions from the statistical decay model GEMINI (see text) for the 50,80 , and $100 \mathrm{MeV} / \mathrm{u}{ }^{139} \mathrm{La}+$ ${ }^{12} \mathrm{C}$ reactions. The total experimental cross sections are plotted for those $\mathrm{Z}$-values near symmetry (solid points). For fragments from more asymmetric decay, in which nonequilibrium processes are expected to contribute to the measured cross sections, only the $90^{\circ}$ symmetric portion of the cross section is plotted (open circles). The statistical model predictions are shown as the histograms. 


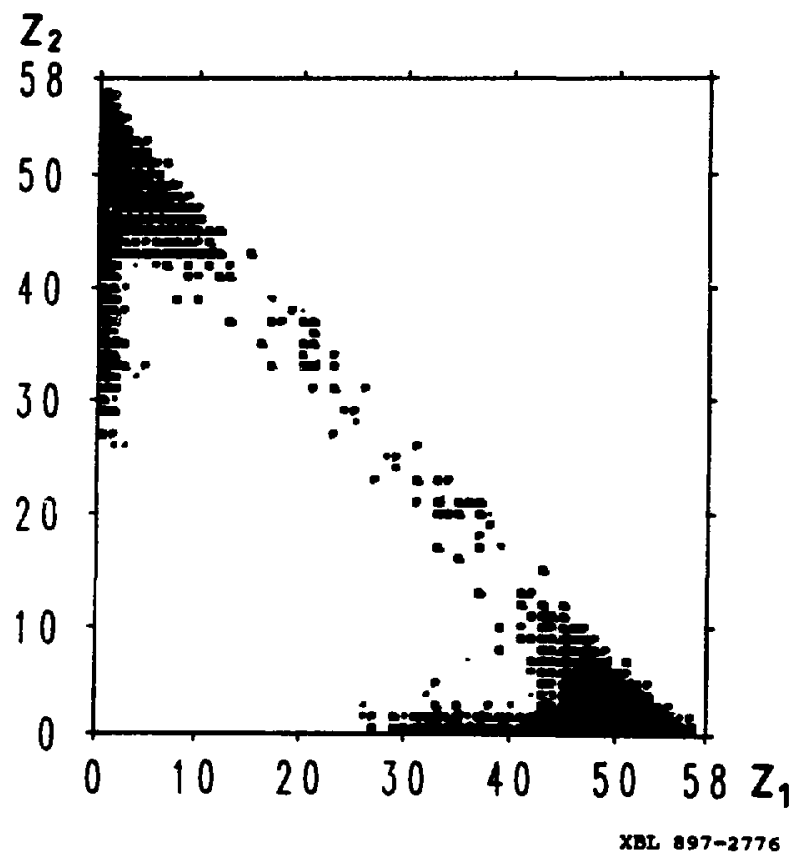

Figure III.26 Density plot of the relative yield (logarithmic scale) of the two heaviest fragments $\left(Z_{1}, Z_{2}\right)$ in the simulated statistical multifragmentation of ${ }^{146} \mathrm{Nd}$ at an excitation energy of $E^{*}=284$ and angular momentum of $39 \leq \mathrm{J} \leq 86$. The two axes give the $Z_{1}$ and $\mathrm{Z}_{2}$ of the two heaviest fragments in each event [Gr 88]. 


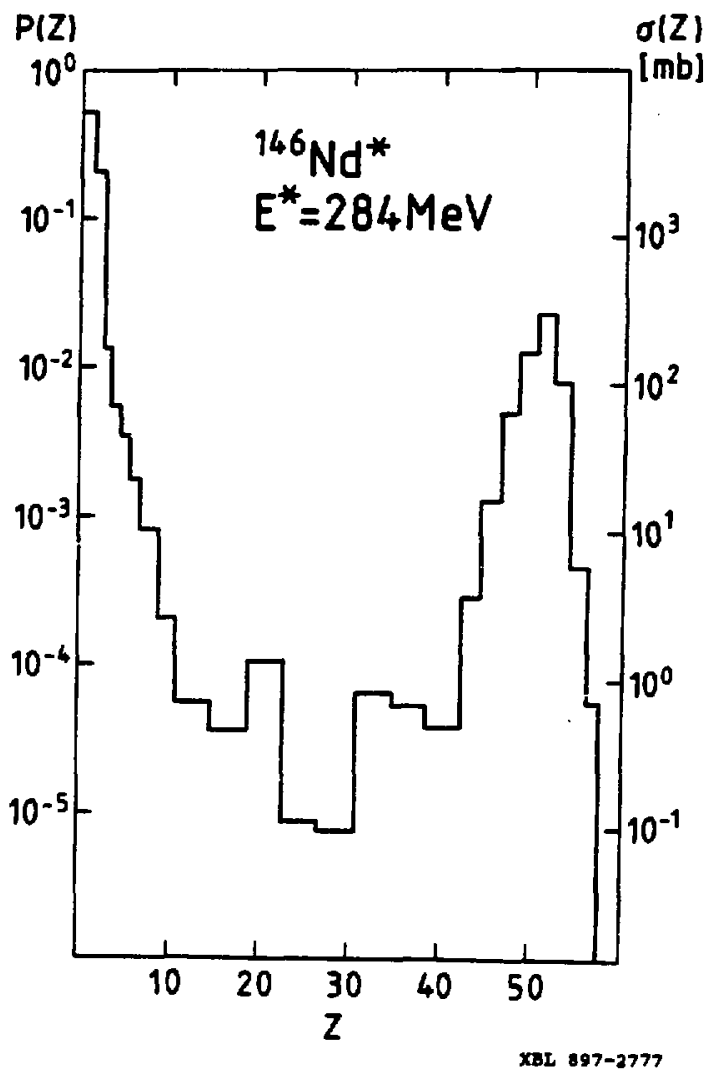

Figure III.27 Charge yield, $d \sigma / d Z$, in mb from the same calculation as in Figure III.26. The total cross section was taken to be $1.73 \mathrm{~b}$. The left scale gives the probability $P(Z)[G r$ 88]. 


\section{IV. $80 \mathrm{MEV} / \mathrm{U}^{139} \mathrm{La}+{ }^{27} \mathrm{Al},{ }^{n 24} \mathrm{Cu},{ }^{197} \mathrm{Au}$ RESULTS}

In this Chapter we will compare the results from the reactions of $80 \mathrm{MeV} / \mathrm{u}{ }^{139} \mathrm{La}+$ ${ }^{27} \mathrm{Al}$, nar $\mathrm{Cu}$, and ${ }^{197} \mathrm{Au}$ to the previously discussed ${ }^{139} \mathrm{La}+{ }^{12} \mathrm{C}$ data. As before, we will begin by examining the global results. We will then tum to the more quantitative results: source rapidities, emission velocities, angular distributions, and cross sections. Next, we will examine the coincidence data. Finally, we will discuss the application of the incomplete fusion - statistical emission model to these systems.

In the discussion of the ${ }^{139} \mathrm{La}+{ }^{12} \mathrm{C}$ reactions, we showed that most complex fragments are produced in highly equilibrated binary decay processes. These processes can be unequivocably associated with compound nucleus decay and deep-inelastic reactions at bombarding energies $\leq 50 \mathrm{MeV} / \mathrm{u}$, and they may include a substantial portion of compound nucleus decay at the higher bombarding energies. We shall see that the multibody nature of the reactions with the heavier targets allows no such simple interpretations. Hence, we have chosen to discuss all of these reactions simultarieously, to present the experimental data, and to focis on the differences between the targets. We are not able at this time to draw strong conclusions about the reaction mechanism.

\section{A. Slobal Results}

1. Coincidence Charge and Rapidity Distributions

Linear contour plots of the two-fold coincidence events $\left(Z_{2}\right.$ versus $\left.Z_{1}\right)$ are shown in Figure IV.1 for the $80 \mathrm{MeV} / \mathrm{u}{ }^{139} \mathrm{La}+{ }^{12} \mathrm{C},{ }^{27} . \mathrm{il}$, nat $\mathrm{Cu}$, and ${ }^{197} \mathrm{Au}$ reactions. The diagonal dashed lines in this figure indicate the charge of the ${ }^{139} \mathrm{La}$ projectile (57). As discussed in the previous chapter, the detection efficiency as determined from Monte Carlo simulations is biased toward symmetric and nearly symmetric coincidence events, so the contours are not representative of the quantitative yields of products. The distributions have been reifiected about the line $Z_{1}=Z_{2}$ to eliminate the bias in the choice of $Z_{1}$ introduced by the asymmetric detector configuration. 
A band due to binary decay, in which the two coincident fragments contain the bulk of the charge, dominates the ${ }^{139} \mathrm{La}+{ }^{12} \mathrm{C}$ contour plot. The distribution of events within the ${ }^{139} \mathrm{La}+{ }^{27} \mathrm{Al}$ contour plot is very broad. The maximum in this plot corresponds to residues of approximately $Z=30$ in coincidence with light complex fragments. For the ${ }^{139} \mathrm{La}+{ }^{\text {nat }} \mathrm{Cu}$ and ${ }^{197} \mathrm{Au}$ reactions, the total detected charge is substantially smaller. Coincidences between very light ecmplex fragments dominate these two plots. The trend is clear: with increasing target mass the coincident fragments typically become smaller.

The total detected charge distributions $\left(Z_{1}+Z_{2}\right)$ are shown in Figure IV.2. The decrease in the average charge and the increase ity the width of the distributions with increasing target mass is much more dramatic than that produced by increasing the bombarding energy in the ${ }^{139} \mathrm{La}+{ }^{12} \mathrm{C}$ reaction. While there are some "binary" events in each of the subplots in Figure III.2 with a total charge near that of the ${ }^{139} \mathrm{La}$ projectile, the fraction of events with $Z_{1}+Z_{2}>50$ to the total number of events derreases with increasing target mass.

The disappearance of the binary signature in these reactions with the heavier targets suggests an increase in the average light charged particle and/or complex fragment multiplicity. An increase in multiplicity should correlate with an increase in the deposited energy. We can naively, and perhaps correctly, attribute the increase in multiplicity to the increase in center-of-mass energy (see Table IV.1).

The source rapidity distributions determined from the coincidence events (see Appendix $\mathrm{D}$ ) are shuwn in Figure IV.3. The average source rapidity is very similar for the ${ }^{139} \mathrm{La}+{ }^{27} \mathrm{Al}$ and ${ }^{139} \mathrm{La}+{ }^{n 24} \mathrm{Cu}$ reactions. While the centroids of the two distributions are the same, the width of the ${ }^{139} \mathrm{La}+{ }^{n a t} \mathrm{Cu}$ distribution is approximately $40 \%$ larger than the width of the ${ }^{139} \mathrm{La}+{ }^{27} \mathrm{Al}$ distribution (which itself is about $60 \%$ larger the the width of the ${ }^{139} \mathrm{La}+{ }^{12} \mathrm{C}$ rapidity distribution, see Figure III.20). This increase in widths may be due to the larger range of impact parameters available with increasing target mass. In the incomplete fusion model described in Appendix $\Pi$, more symmetric systems with larger 
ranges of impact parameters give rise to sources with broader rapidity distributions.

2. Z Versus Velocity Plots

Figures IV.4 shows contours of the Lorentz-invariant cross section, $\frac{d^{2} \sigma}{\gamma^{4} v^{2} d \Omega d v}$, in the $\mathrm{Z}$ - velocity plane for the ${ }^{139} \mathrm{La}+{ }^{27} \mathrm{Al}$ reaction. As with the ${ }^{139} \mathrm{La}+{ }^{12} \mathrm{C}$ reactions (Figure III.4 - 6), there are three components in these contour plots. The first component, which dominates the contour plot at $2.5^{\circ}$, consists of heavy residues of $Z>30$ and $\mathrm{V} / \mathrm{V}_{\text {beam }} \sim 1$. The second component, which dominates at $6.5^{\circ}$ and $8.5^{\circ}$, consists of anisotropic complex fragments of $Z<10$ and $V / V_{\text {beam }}<0.9$. These two components have about equal intensities at $4.5^{\circ}$. The third component consists of the fragments along the two Coulomb ridges, which are characteristic of relaxed binary decay. While these Coulomb ridges are broader than those in the ${ }^{139} \mathrm{La}+{ }^{12} \mathrm{C}$ reactions (note the difference in the distributions at $4.5^{\circ}$ in Figures III.4 and IV.4) they are still clearly visible at $2.5^{\circ}$ and 4.5. This broadening may be due to the increase in light particle or complex fragment multiplicity, to the broader source rapidity distribution, or to a combination of these effects.

Lorentz-invariant cross sections in the $\mathrm{Z}$ - velocity plane for the ${ }^{139} \mathrm{La}+{ }^{\text {nat }} \mathrm{Cu}$ reaction are shown at several laboratcry angles in Figure IV.5. The residue charge distribution extends down to slightly smaller $\mathrm{Z}$-values $(\sim 15)$ than in the ${ }^{139} \mathrm{La}+{ }^{27} \mathrm{Al}$ reaction, but the residue velocity distributions are nearly identical in both cases. The anisotropic complex fragment component in the ${ }^{139} \mathrm{La}+$ natc $\mathrm{Cu}$ reaction is also quite similar to the corresponding components in the ${ }^{139} \mathrm{La}+{ }^{12} \mathrm{C}$ and the ${ }^{139} \mathrm{La}+{ }^{27} \mathrm{Al}$ reactions. The difference between the ${ }^{139} \mathrm{La}+$ nalCu contour plots in Figure IV.5 and those for the lighter targets is that there is no longer any evidence of the Coulomb ridges. Instead, the residues, which move at slightly less than beam velocity, merge smoothly into the anisotropic component near $Z=15$. Of course, the Coulomb ridge could still exist but be buried under the two more abundant components. 
3. $\mathrm{Y}-\mathrm{P}_{\perp} / \mathrm{mc}$ Plots

Density plots of $\frac{\partial^{2} \sigma}{\partial \mathrm{Y} \partial\left(\mathrm{P}_{\perp} / \mathrm{mc}\right)}$ in the rapidity - perpendicular momentum plane are shown in Figure IV.6 for representative $\mathrm{Z}$-values in the ${ }^{139} \mathrm{La}+{ }^{27} \mathrm{Al}$ reaction. As with the invariant $Z$ - velocity maps described above, these plots are similar to those observed with the ${ }^{12} \mathrm{C}$ target. The fragments of $\mathrm{Z}<20$ appear to be emitted primarily with Coulomb velocities. The angular distributions change from backward peaked for fragments of $\mathrm{Z}<$ 14 , through forward/backward symmetric near $Z=14$, to forward peaked for the heavier Z-values.

The patterns observed in these density plots are slightly more elliptical than those observed in the ${ }^{139} \mathrm{La}+{ }^{12} \mathrm{C}$ reactions. This increase in ellipticity is probably due to the broader source rapidity distribution in the ${ }^{139} \mathrm{La}+{ }^{27} \mathrm{Al}$ reaction. This behavior has been experimentally observed in the ${ }^{139} \mathrm{La}+{ }^{64} \mathrm{Ni}$ reaction at $18 \mathrm{MeV} / \mathrm{u}$, in which a continuum of sources with a broad range of velocities, each emitting fragments upon characteristic Coulomb circles, generated inclusive distributions in velocity space that were elongated along the beam direction [Co 89].

The elliptical pattems observed in these densiry plots are also slightly broader in emission velocity than those of the ${ }^{139} \mathrm{La}+{ }^{12} \mathrm{C}$ reaction. The increased broadening may be due to fragment recoil effects associated with the increased light particle and complex fragment multiplicity, and to the broader source rapidity distribution.

The $\frac{\partial^{2} \sigma}{\partial Y \partial\left(P_{\perp} / m c\right)}$ density plots of the heavier $Z$-values, $Z>25$, are somewhat different than the plots of similar fragments in the ${ }^{139} \mathrm{La}+{ }^{12} \mathrm{C}$ reactions. They do not appear to have the well-defined ring-shaped distributions characteristic of binary decay, but rather to have filled, disk-shaped distributions. These types of distributions suggest a nonbinary decay mechanism. Similar experimental distributions have been attributed to projectile fragmentation reactions [Go 74], and have been fit with Gaussian functions of the 
form [Da 86]:

$$
\frac{\partial^{2} \sigma}{\partial \mathrm{Y} \partial\left(\mathrm{P}_{\perp} / \mathrm{mc}\right)}=\mathrm{C} \exp \left[-\frac{\left(\mathrm{P}_{\perp} / \mathrm{mc}\right)^{2}}{2 \sigma_{\perp}{ }^{2}}\right] \exp \left[-\frac{\left(\mathrm{Y}-\mathrm{Y}_{0}\right)^{2}}{2 \sigma_{\| 1}^{2}}\right] .
$$

The projectile fragmentation interpretation has not explained the widths $\left(\sigma_{\perp}\right.$ and $\left.\sigma_{\|}\right)$of the fragment momentum distributions in the intermediate energy regime due, in part, to its neglect of the Coulomb force [Wo 82]. Additional broadening of the momentum distributions, presumably due to collective energy dissipation, has also been observed $\mathrm{Da}$ 86].

Density plots of $\frac{\partial^{2} \sigma}{\partial \mathrm{Y} \partial\left(\mathrm{P}_{\perp} / \mathrm{mc}\right)}$ in the rapidity - perpendicular momentum plane are shown in Figure IV.7 for representative $\mathrm{Z}$-values in the ${ }^{139} \mathrm{La}+{ }^{\text {nat }} \mathrm{Cu}$ reaction. In general, these density plots do not resemble those of the lighter targets. The distributions are not elliptical; the Coulomb ring is filled in for $Z \geq 10$. These fragments are not emitted with a well-defined velocity in quasi-binary reactions. Instead, the broadening associated with higher multiplicities of light particles and complex fragments, and the broader source rapidity distribution, have completely obliterated the characteristic Coulomb rings for fragments of $6<Z<20$. For fragments of $Z>20$, the distributions appear to be of the form of (25) above.

Although for the heavier fragments the population of events in the $\mathrm{Y}-\mathrm{P}_{\perp}$ plane depends quite strongly upon the target mass, it is interesting to compare the density plots for $Z$ = 6 fragments (Figures III.7, III.8, IV.5, and IV.6). While the distributions lonk more circular and less broad with the lighter targets, the population of the velocity plane is quite similar in each of the reactions. The bulk of the emission is Coulomb-like at backward angles in the source frame.

There are some non-physical background components in these $\frac{\partial^{2} \sigma}{\partial \mathrm{Y} \partial\left(\mathrm{P}_{\perp} / \mathrm{mc}\right)}$ 
density plots that should be pointed out. The first background component is visible as the tailing to small rapidities for heavier fragments $(Z>20)$, which has about the same intensity in the ${ }^{139} \mathrm{La}+{ }^{12} \mathrm{C},{ }^{27} \mathrm{Al}$, and ${ }^{\text {nat }} \mathrm{Cu}$ reactions. We associate these events with incomplete charge collection in the $5 \mathrm{~mm} \mathrm{Si(Li)} \mathrm{detectors.} \mathrm{This} \mathrm{incomplete} \mathrm{charge}$ collection is due to radiation induced damage of the detectors [Kr 84] or to impurities in the crystal. These events are typically a small percentage of the total number of events.

The second background component is visible as the tailing to large rapidities for fragments of $Z \leq 10\left(Z \leq 14\right.$ in the $100 \mathrm{MeV} / \mathrm{u}^{139} \mathrm{La}+{ }^{12} \mathrm{C}$ reaction $)$. This component is more intense for the reactions with the ${ }^{27} \mathrm{Al}$ and nat $\mathrm{Cu}$ targets than for those with the ${ }^{12} \mathrm{C}$ target, and more intense at $100 \mathrm{MeV} / \mathrm{u}$ than at $80 \mathrm{MeV} / \mathrm{u}$ in the ${ }^{139} \mathrm{La}+{ }^{12} \mathrm{C}$ reaction. These events correspond to light particle - complex fragment double hits in a single array telescope. The $\mathrm{Z}$ identification of the fragments is done solely with the $300 \mu \mathrm{Si}$ and $5 \mathrm{~mm}$ $\mathrm{Si}(\mathrm{Li})$ elements of the telescope. A proton or alpha particle that strikes a telescope in coincidence with a complex fragment will deposit very litule energy in the $\mathrm{Si}$ and $\mathrm{Si}(\mathrm{Li})$ elements and will not affect the $\mathrm{Z}$ identification. However, the light output of the plastic scintillators is very strongly charge dependent [Mc 88], with heavier ions producing much less light per unit energy than protons. Therefore, the charge-dependent energy calibration of the plastic scintillators associates very large energies to the high light outputs from the coincident light particles, and gives rise to the tailing to large rapidities. The background is much worse in the reactions in which the light charged particle multiplicity is high. We have eliminated this tailing for the higher Z-values by adding the energy deposited in the plastic scintillators only for "agments that have sufficient range to punch-through the $\mathrm{Si}$ and $\mathrm{Si}(\mathrm{Li})$ detectors. This cut-off was taken to be $\mathrm{Z}>\mathrm{i} 0$ for the reactions at $80 \mathrm{MeV} / \mathrm{u}$ and $\mathrm{Z}>14$ for the ${ }^{139} \mathrm{La}+{ }^{12} \mathrm{C}$ resction at $100 \mathrm{MeV} / \mathrm{L}$.

B. Quantitative Results

1. Source Rapidities Inclusive source rapidities for the $80 \mathrm{MeV} / \mathrm{u}{ }^{139} \mathrm{La}+{ }^{27} \mathrm{Al}$ reaction were extracted 
from elliptical fits to the distributions in the $\frac{\partial^{2} \sigma}{\partial \mathrm{Y} \partial\left(\mathrm{P}_{\perp} / \mathrm{mc}\right)}$ density plots as described in Appendix I (equitions A.16 - A22). Figure III.11 shows the ratio of the major to minor axes of the titted ellipses. The distributions from the ${ }^{27} \mathrm{Al}$ target are more elliptical than those from the $80 \mathrm{MeV} / \mathrm{u}{ }^{139} \mathrm{La}+{ }^{12} \mathrm{C}$, and are slightly more elliptical than the $100 \mathrm{MeV} / \mathrm{u}$ ${ }^{139} \mathrm{La}+{ }^{12} \mathrm{C}$ distributions. This is indicative of a broader source rapidity distribution, and presumably due tc the larger range of impact parameters that can lead to complex fragment emission with a heavier target.

The source rapidities were also determined from the center-of-mass rapidities of the 2- and 3-fold coincidence events (see Appendix I). The measured source rapidities are shown $:$ Figure IV.8. For the ${ }^{139} \mathrm{La}+{ }^{27} \mathrm{Al}$ reaction, it is apparent that there is a systentatic difference between the inclusive and coincidence source rapidities both in the magnitude and in the trend of the data. The inclusive data show that the lighter fragments are emitted from slower sources, while the coincidence data show no depencience of the source rapidity upon $Z$-value for $Z<40$.

While there may be sys:-matic errors associated with the coinsidence data due to efficiency effects, it is more likely that the difference between the source rapidities is due to a systematic error in the inclusive extraction procedure. There are larger fluctuations in the source rapidities exuracted from the elliptical fits than in those determined from the coincidence data both in the $80 \mathrm{MeV} / \mathrm{u}^{139} \mathrm{La}+{ }^{27} \mathrm{Al}$ and the $100 \mathrm{MeV} / \mathrm{u}{ }^{139} \mathrm{La}+{ }^{12} \mathrm{C}$ reactions (see Figure III.9). As the elliptical distibutions in the $\frac{\partial^{2} \sigma}{\partial Y \partial\left(P_{\perp} / m c\right)}$ density plots fill in, it becomes very difficult to extract meaningful quantities from them. On the other hand, Monte Carlo simulations have indicated that the coincidence detection efficiency is only very weakly dependent upon the source velocity, so there should be no strong biases in determining the source rapidities from the coincidence events.

For the nar $\mathrm{Cu}$ target, the source rapidities were determined solely from the 2- and 3- 
fold coincidence events because the inclusive distributions in the $\frac{\partial^{2} \sigma}{\partial \mathrm{Y} \partial\left(\mathrm{P}_{\perp} / \mathrm{mc}\right)}$ density plots do not resemble ellipses. The measured source rapidities are shown in Figure III.9. We observe a slight dependence of the source rapidity upon the fragment Z-value, with the lighter fragments tending to come from slower sources. In reverse kinematics, slower sources are associated with larger momentum transfers and energy depositions. The coincidence data (Figure IV.1) show that the heavy fragments are ustally in coincidence with light fragments, while the light fragments tend to be in coincidence with other light fragments. In general, the heavy-light coincidences have larger source repidities and correspondingly smaller momentum transfers and energy depositions than the light-light coincidences.

The average source rapidities determined from the coincidence events are very similar in the ${ }^{139} \mathrm{La}+{ }^{27} \mathrm{Al}$ and ${ }^{139} \mathrm{La}+$ nat $\mathrm{Cu}$ reaccions. At lower bombarding energy, the average source rapidity was able to characterize products from incomplete fusion reactions by allowing the extraction of mass transfers, momentum transfers, and energy depositions. This is not the case in the $80 \mathrm{MeV} / \mathrm{u}^{139} \mathrm{La}+{ }^{27} \mathrm{Al}$ and ${ }^{139} \mathrm{La}+{ }^{\text {ma }} \mathrm{Cu}$ reactions where the average source rapidities are quite similar, hut other experimental observables (cross sections and coincidence charge distributions) are very different.

\section{Emission Velocities}

The fragment emission velocities are shown in Figure IV.9 as determined from the inclusive and coincidence data in the ${ }^{139} \mathrm{La}+{ }^{27} \mathrm{Al}$ and ${ }^{\text {nat }} \mathrm{Cu}$ reactions. Ti:s inclusive emission velocities were extracted from the minor axes of the elliptical fits to the distributions in the ${ }^{139} \mathrm{La}+{ }^{27} \mathrm{Al} \frac{\partial^{2} \sigma}{\partial \mathrm{Y} \partial\left(\mathrm{P}_{\perp} / \mathrm{mc}\right)}$ density plots. The coincidence emission velocities were determined from the velocities in the center-of-mass frame using equations (A25 - A34). The emission velocities decrease with increasing fragment charge in both reactions. This shows that the major influences on these velocities are Coulomb repulsion 
and momentum conservation.

As with the extracted source rapidities, there is a systematic disagreement between the emission velocities determined from the inclusive and the coincidence data. The inclusive emission velocities are measured relative to the average source rapidity, which, by definition, lies along the beam direction. In contrast, the emission velocities determined from the coincidence data are measured in the center-of-mass of each event, which needs not be along the beam direction (see Figure IV.22). The coincidence data are strongly biased by the detection efficiency, which depends upon the directions of the fragment emission vector and the center-of-mass vector. On the other hand, there are smaller fluctuations in the emission velocities extracted from the inclusive data, so the inclusive emission velocities should be less biased than the coincidence velocities. Unfortunately, it was not possible to determine emission velocities from the inclusive data in the ${ }^{139} \mathrm{La}+$ nar $\mathrm{C} u$ reaction since, in general, the inclusive distributions in the $\frac{\partial^{2} c}{\partial \mathrm{Y} \partial\left(\mathrm{P}_{\perp} / \mathrm{mc}\right)}$ density plots could not be fit to ellipses.

In Figure IV. 10 the emission velocities of fragments in the $80 \mathrm{MeV} / \mathrm{u}{ }^{139} \mathrm{La}+{ }^{12} \mathrm{C}$, ${ }^{27} \mathrm{Al}$, and nat $\mathrm{Cu}$ reactions are shown as determined from the 2-fold coincidence events. The trend is similar to that seen in the ${ }^{139} \mathrm{La}+{ }^{12} \mathrm{C}$ system. An increase in the center-ofmass energy leads to a smaller emission velocity for a given $Z$-value. As described in the previous section, this effect could be due to an increase in light particle emission either before, after, or simultaneously with the complex fragment emission, or it could be due to the expansion of the system with increased temperature, or to a combination of these effects. In addition, complex fragment multiplicities greater than two could also decrease the average emission velocity for a given fragment relative to the binary emission velocity.

The similarities of the emission velocities at the smallest and largest Z-values for all of the targets in Figure IV.10 may be due to efficiency effects. We expect to sample only a portion of the emission velocity distributions for the very light and the very heavy complex 
fragments. The heaviest fragments are confined to a small angular region about the beam, and only fragments emitted to laboratory angles larger than $2.2^{\circ}$ are obseved. These fragments have the largest emission velocities Since the lighter fragments can be emitted to angles beyond the limit of the detector coverage $\left(9^{\circ}\right)$, there is a corresponding bias to detect fragments with the smallest emission velocities. For the lightest and heaviest fragments, the measured emission velocities may be less dependent on the actual velocity distribution than on the detector geometry. Thus the measured emission velocities should be strongly biased.

Figures IV.11 and IV.12 show the emission velocity distributions of $Z=6$ fragments in the frame corresponding to the average source rapidity for the $80 \mathrm{MeV} / \mathrm{L}{ }^{139} \mathrm{La}$ $+{ }^{27} \mathrm{Al}$ and nart $\mathrm{Cu}$ reactions. The distributions at $25^{\circ}$ and $155^{\circ}$ for the ${ }^{139} \mathrm{La}+{ }^{27} \mathrm{Al}$ reaction (Figure IV.11) have similar shapes. At $165^{\circ}$ the distribution is considerably broader due to a tailing to larger emission velocities (backwards in the laboratory). For the ${ }^{139} \mathrm{La}_{2}+$ matCu reaction (Figure IV.12) the centroids and widths of the distributions increase as the emission angle increases. This tailing at backward angles for target-like complex fragments is characteristic of lower energy reverse kinematics reactions, but is not observed in the ${ }^{139} \mathrm{La}+{ }^{12} \mathrm{C}$ system at 80 and $100 \mathrm{MeV} / \mathrm{u}$.

The origin of these fragments is less clear in the reactions at $80 \mathrm{MeV} / \mathrm{u}$ than at lower energy. As discussed in the Introduction, at bombarding energies $\leq 50 \mathrm{MeV}$ iu these fragments are produced in the binary deep-inelastic mechanism. Studies with coincident fission fragments indicate that they are associated with smaller energy depositions and momentum transfers than the equilibrium compound nucleus component [Fa 87], and that they probably result from larger impact parameters. The coincidence data suggest that most of the light fragments in the $80 \mathrm{MeV} / \mathrm{u}{ }^{139} \mathrm{La}+{ }^{27} \mathrm{Al}$ and ${ }^{139} \mathrm{La}+{ }^{n a t} \mathrm{Cu}$ reactions are produced in events in which the multiplicities of light particles and/or complex fragments can be large. Whether these fragments are still produced by deep-inelastic reactions followed by a substantial amount of sequential decay, or whether they are instead remnants 
of a more violent reaction mechanism, such as the fireball mechanism described above, remains to be determined.

\section{Angular Distributions}

Fragment angular distributions in the frame corresponding to the average source rapidity are shown in Figure IV.13 for the ${ }^{139} \mathrm{La}+{ }^{27} \mathrm{Al}$ and ${ }^{139} \mathrm{La}+{ }^{\text {nat }} \mathrm{Cu}$ reactions. We observe backward peaked angular distributions for the smaller Z-values which evolve smoothly through forward/backward symmetry and then become forward peaked for the larger $\mathrm{Z}$-values. The shapes of the ${ }^{139} \mathrm{La}+{ }^{27} \mathrm{Al}$ and ${ }^{139} \mathrm{La}+{ }^{n 2 t} \mathrm{Cu}$ angular distributions are very similar to each other and also to the ${ }^{139} \mathrm{La}+{ }^{12} \mathrm{C}$ angular distributions (Figure II.17). Fonward/backward symmetric angular distributions occur near $Z=16$ for both the ${ }^{27} \mathrm{Al}$ and the narcu targets, which is at a slightly smaller $\mathrm{Z}$-value than the forward/backward symmetric angular distributions in the ${ }^{139} \mathrm{La}+{ }^{12} \mathrm{C}$ reaction.

The ratios of the cross sections in the forward hemisphere to those in the backward hemisphere are shown in Figure IV.14 for the $80 \mathrm{MeV} / \mathrm{u}$ reactions. The trend of the ratios is similar: with increasing $Z$-value the ratios increase exponentially. There are two slight dependences on the target mass: the Z-value at which the cross sections are forward/backward symmetric decreases with increasing target mass, and the ratios show a stronger dependence on 2-value as the target mass is increased. The increase in the anisotropy of the angular distributions as the target mass is increased suggests a greater amount of non-equilibrium emission with the heavier targets, since statistical decay products (from well-defined sources) have forward/backward symmetric angular distributions.

\section{Cross Sections}

Figure IV.15 shows the charge distribution systematics for the ${ }^{139} \mathrm{La}+{ }^{27} \mathrm{Al}$ system. At $18 \mathrm{MeV} / \mathrm{u}$, the distribution has a shape determined by the potential energy of the two decay partners; there is a maximum in the yield at symmetry because the system is above the Businaro-Gallone point [Bu 55], and the potential energy for symmetric decay is 
smaller than at intermediate asymmetries (see Figure I.1). At $47 \mathrm{MeV} / \mathrm{u}$ the charge distribution has evolved into a $U$ shape, which is fairly flat near symmetry. Perhaps more interestingly, the cross sections near symmety decrease significantly between 18 and 47 $\mathrm{MeV} / \mathrm{u}$. The charge distribution at $80 \mathrm{MeV} / \mathrm{u}$ resembles the charge distribution in the 47 $\mathrm{MeV} / \mathrm{u}$ reaction. The yields for fragments of $\mathrm{Z}<20$ are slightly larger than those at 18 $\mathrm{MeV} / \mathrm{u}$, while the more symmetric products have somewhat smaller yields. The evolution of the ${ }^{139} \mathrm{La}+{ }^{27} \mathrm{Al}$ charge distribution with increasing bombarding energy is similar to that observed in the ${ }^{139} \mathrm{La}+{ }^{12} \mathrm{C}$ system. The decrease in the cross sections for symmetric products between 18 and $47 \mathrm{MeV} / \mathrm{u}$, while contrary to what is generally expected, may be due to the onset of incomplete fusion as described above.

Charge distributions from the 47 and $80 \mathrm{MeV} / \mathrm{u}{ }^{139} \mathrm{La}+{ }^{\text {nat }} \mathrm{Cu}$ reactions are shown in Figure IV.16. The shapes of the two distributions are very similar and the difference in the yields of approximately $20 \%$ is within the possible systematic error associated with the absolute cross section determination. In Figure IV.17 the cross sections at $80 \mathrm{MeV} / \mathrm{u}$ for the reactions on ${ }^{12} \mathrm{C},{ }^{27} \mathrm{Al}$, and nat $\mathrm{Cu}$ are shown. There is a factor of ten difference in the yields between the ${ }^{12} \mathrm{C}$ and ${ }^{27} \mathrm{Al}$ targets. This same factor has been observed between the ${ }^{139} \mathrm{La}+{ }^{12} \mathrm{C}$ and ${ }^{139} \mathrm{La}+{ }^{27} \mathrm{Al}$ yields at both 18 and $47 \mathrm{MeV} / \mathrm{u}$. There is another factor of two increase in the cross section between the ${ }^{27} \mathrm{Al}$ and narCu targets. Table $\mathrm{NV} .1$ shows that the differences in geometric overlap of the three systems $\left(R_{1}+R_{2}\right)$ are not nearly large enough to account for the differences in cross section. This may be additional indirect evidence of the higher complex fragment multiplicities in the reactions with the heavier targets.

\section{Coincidence Data}

Figure IV.18 shows the total coincidence charge distributions for different multiplicities of complex fragments. For purpose of comparison. the maxima of the distributions have been normalized to each other. The total detected charge in the ${ }^{139} \mathrm{La}+{ }^{27} \mathrm{Al}$ reaction does not depend strongly upon the complex fragment multiplicity. 
This shows that most of the charged particle loss is in the form of light particles and that the complex fragment multiplicity is usually no greater than two. If the 2 -fold events were in fact multibody events in which many complex fragments escaped undetected, then we would expect the higher-fold distributions to have a larger average charge. The similarity of the distributions indicates that only a small amount of charge can, on the average, escape as an undetected complex fragment (such as in a Li or Be fragment) in the 2-fold coincidence events. The total charge distributions in the ${ }^{139} \mathrm{La}+{ }^{12} \mathrm{C}$ reactions are also independent of the complex fragment multiplicity (see Figure III.19).

In contrast, the coincidence charge distributions in Figure IV.18 for the reactions with the nat $\mathrm{Cu}$ and ${ }^{197} \mathrm{Au}$ targets do depend strongly upon the complex fragment multiplicity. This indicates that the 2-fold events are not truly 2-body, but rather are events in which one or more complex fragment remain undetected. The difference between the 3and 4 body distributions suggests that the most probable exit channel in the ${ }^{139} \mathrm{La}+{ }^{\text {nat }} \mathrm{Cu}$ reaction has at least 4 complex fragments. The ${ }^{197} \mathrm{Au}$ target is similar to the nat $\mathrm{Cu}$ target in that there is a large difference between the 2- and 3-fold distributions. For the ${ }^{197} \mathrm{Au}$ target the average multiplicity is at least three, and very likely higher.

In contrast to the total charge distributions, Figure IV.8 shows that the center-ofmass rapidity distributions do not depend upon the number of detected complex fragments. In the ${ }^{139} \mathrm{La}+{ }^{27} \mathrm{Al}$ reaction, where the complex fragment multiplicity is usually low, this independence indicales the common origin of all of the complex fragments. The 3- and 4 fold events are not a special class of events with larger momentum transfers and energy depositions. The independence of the rapidity distributions in the ${ }^{139} \mathrm{La}+{ }^{n a t} \mathrm{Cu}$ and ${ }^{139} \mathrm{La}$ $+{ }^{197} \mathrm{Au}$ reactions suggests that the 2 - and 3-fold events correspond to high multiplicity events in which one or more fragments remain undetected.

The centroids and widths of the rapidity and total charge distributions are shown in Figure $\mathrm{IV} .19$ for 2 - and 3-fold coincidence events. The average source rapidity decreases slightly when the target mass is increased from 12 to 27 , but then remains approximately 
constant between 27 and 197. The widths of the rapidity distributions, which are indicated by the bars through the points, show a monotonic increase with target mass. The centroids of the total charge distributions decrease and the widths increase with increasing target mass, suggesting the increase in light particle and complex fragment multiplicity mentioned above.

In the incomplete fusion model, a single measurement of the source velocity allows the determination of the mass transfer, momentum transfer and the excitation energy deposited in the incomplete fusion product. The angular momentum of the incomplete fusion product is assumed to be determined by the mass transfer, and a similar mass transfer should give rise to an equivalent angular momentum deposition. Observables such as the emission velocities, the total charge detected in the coincidence events, and most importantly, the cross sections are used to verify the extracted quantities. For the ${ }^{139} \mathrm{La}+{ }^{27} \mathrm{Al}$, nare $\mathrm{Cu}$, and ${ }^{197} \mathrm{Au}$ reactions, we observe differences in the emission velocities, the coincidence charge distributions, the inferred complex fragment multiplicities, and the cross sections, but the average source rapidities are very sumilar. Hence the source rapidity is not able to characterize the ${ }^{139} \mathrm{La}+{ }^{\text {nat }} \mathrm{Cu}$ and ${ }^{139} \mathrm{La}+{ }^{197} \mathrm{Au}$ reactions as it has for reactions at lower bombarding energy. This suggests that the incomplete fusion model is not applicable to these systems, and that the reactions are proceeding through another mechanism.

Figure IV.20 and IV.21 illustrate the same point. In the ${ }^{139} \mathrm{La}+{ }^{64} \mathrm{Ni}$ reaction at 18 MeV/u one was able, by gating on specific source rapidities, to pick out specific amounts of mass transfer [Co 89]. However, the contour plots in Figures IV.20 and IV.21 show that there is no dependence of the total detected charge on the source rapidity. The source rapidity becomes very broad for small values of the total detected charge in the ${ }^{139} \mathrm{La}+$ ${ }^{n a t} \mathrm{Cu}$ reaction. As mentioned above, this is because these reactions have, on the average, at least 4 complex fragments in the exit channel, and the broadening is probably due to $\mathrm{mi}$ sing the momentum of at least one large fragment. 


\section{Model Calculations}

As described in the Introduction, the compound statistical decay model has had only moderate success in reproducing experimental results for ${ }^{139} \mathrm{La}$ and ${ }^{93} \mathrm{Nb}+{ }^{27} \mathrm{Al}$ complete fusion reactions at bombarding energirs $\leq 20 \mathrm{MeV} / \mathrm{u}[\mathrm{Ch} 88 \mathrm{~b}, \mathrm{Ch} 89]$ While the shapes of the charge distributions could be reproduced, the absolute magnitudes of the cross sections were underpredicted by factors of 3 - 5. The additional yield is probably due to quasifission or incomplete fusion reactions at \&-waves larger than $\mathrm{J}_{\text {crit }}$ (see Introduction). With the questionable applicability of the incomplete fusion model to these reactions with heavier targets, we should not expect that the calculations will do a very good job in reproducing the experimental data a: $8,0 \mathrm{MeV} / \mathrm{u}$.

The mass, charge, excitation energy, and spin distribution of the compound nuclei produced in the $80 \mathrm{MeV} / \mathrm{u}{ }^{139} \mathrm{La}+{ }^{27} \mathrm{Al}$ and ${ }^{139} \mathrm{La}+{ }^{\text {nal }} \mathrm{Cu}$ reactions were deduced as described in Chapter III (equations 20,21,24) from the measured coincidence source rapidities of these systems. The input parameters for the statistical emission code GEMINI are given in Table IV.2. In these decay calculations, the $\mathrm{J}_{\max }$ of the triangular spin distribution was taken to be $88 \mathrm{n}$, which is the largest spin at which finite range barriers for symmetric decay exist [Si 86b]. The calculations are shown as the histograms in Figure IV.22. While the shapes of the experimental charge distributions and the calculations are quite different, no attempt has been made to extract an "isotropic" component for these system as was done for the ${ }^{139} \mathrm{La}+{ }^{12} \mathrm{C}$ reactions. The model calculations underpredict the cross sections near symmetry by a factor of 3 in the ${ }^{139} \mathrm{La}+{ }^{27} \mathrm{Al}$ reaction and by a factor of 10 in the ${ }^{139} \mathrm{La}+{ }^{\text {nar }} \mathrm{Cu}$ reaction. This indicates that in these reactions the bulk of the complex fragments, even those near symmetry, are produced in non-equilibrium processes. 
Table IV.1

$$
80 \mathrm{MeV} / \mathrm{u}^{139} \mathrm{La}+\mathrm{X}
$$

$$
\text { Target___. } \quad E_{C M}(\mathrm{MeV}) \quad \sigma_{g e o m}\left(R_{P r o j}+\mathrm{RT}_{\mathrm{Tar}} 2 \quad \frac{\mathrm{Y}_{\text {source }}}{\mathrm{Y}_{\text {beam }}}\right.
$$

$\begin{array}{rccc}{ }^{12} \mathrm{C} & 881 & 9.14 & 0.957 \pm 0.007 \\ { }^{27} \mathrm{Al} & 1798 & 10.01 & 0.938 \pm 0.007 \\ { }^{\text {nat } \mathrm{Cu}} & 3457 & 11.22 & 0.938 \pm 0.007 \\ { }^{197} \mathrm{Au} & 6453 & 13.46 & 0.949 \pm 0.007\end{array}$

Table IV. 2

Statistical Decay Calculations

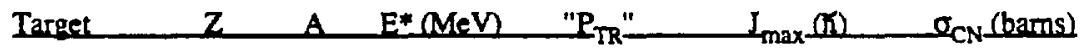

$\begin{array}{rllllll}{ }^{12} \mathrm{C} & 60 & 146 & 500 & 0.57 & 61 & 0.434 \\ { }^{27} \mathrm{Al} & 62 & 149 & 650 & 0.33 & 88 & 0.365 \\ { }^{\mathrm{naC}} \mathrm{Cu} & 62 & 149 & 650 & 0.14 & 88 & 0.234\end{array}$




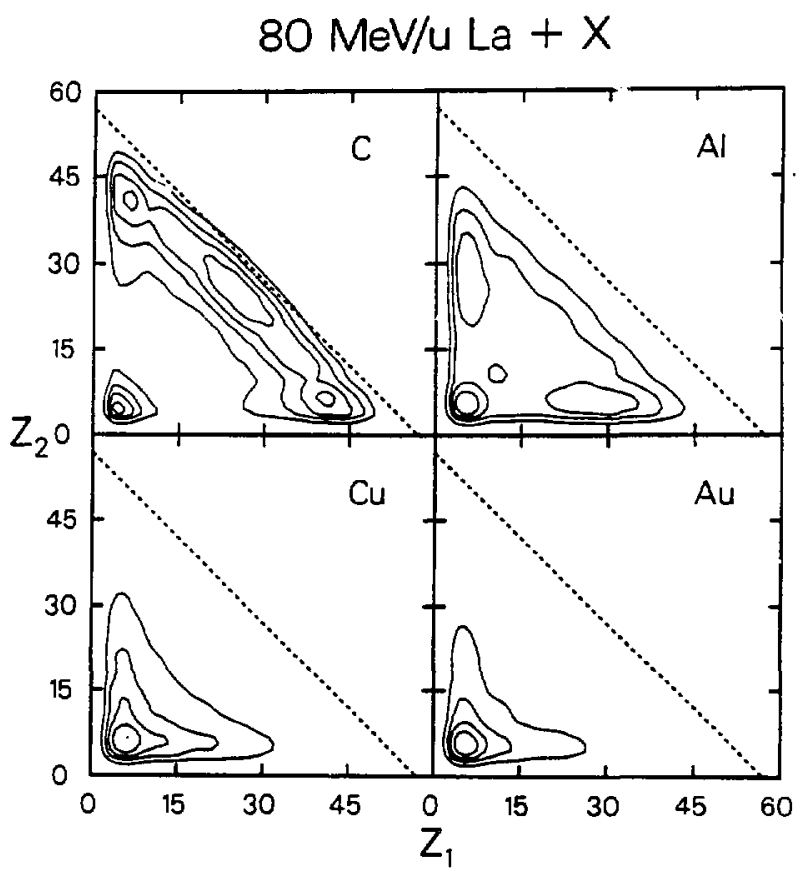

XBL $897-2757$

Figure IV.1 Linear contour plots of 2 -fold $Z_{2}$ versus $Z_{1}$ coincidence events in the 80 $\mathrm{MeV} / \mathrm{u}{ }^{139} \mathrm{La}+{ }^{12} \mathrm{C},{ }^{27} \mathrm{Al}$, nat $\mathrm{Cu}$ and ${ }^{197} \mathrm{Au}$ reactions. The sets of four lines correspond to contours in the relative ratios of 4:3:2:1. The dashed lines indicate the charge of the ${ }^{139} \mathrm{La}$ projectile (57). All of the distributions have been reflected about the line $Z_{1}=Z_{2}$ to remove the bias due to the asymmetric detector configuration. 


\section{$80 \mathrm{MeV} / \mathrm{u} \mathrm{La}+\mathrm{X}$}

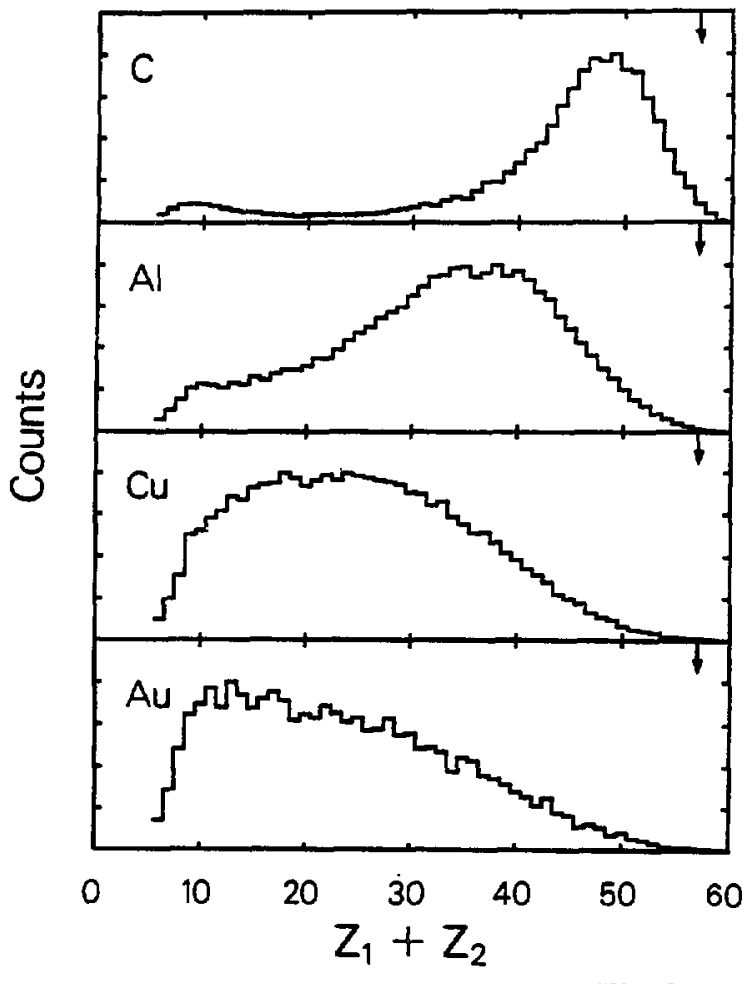

XBL $897-2758$

Figure IV.2 Distributions of the total detected charge $\left(Z_{1}+Z_{2}\right)$ in 2-fold coincidence events for the $80 \mathrm{MeV} / \mathrm{u}{ }^{139} \mathrm{La}+{ }^{12} \mathrm{C},{ }^{27} \mathrm{Al}$, nat $\mathrm{Cu}$, and ${ }^{197} \mathrm{Au}$ reactions. The maxima in each of the distributions have been normalized to each other. The arrows indicate the charge of the ${ }^{139} \mathrm{La}$ projectile (57). 


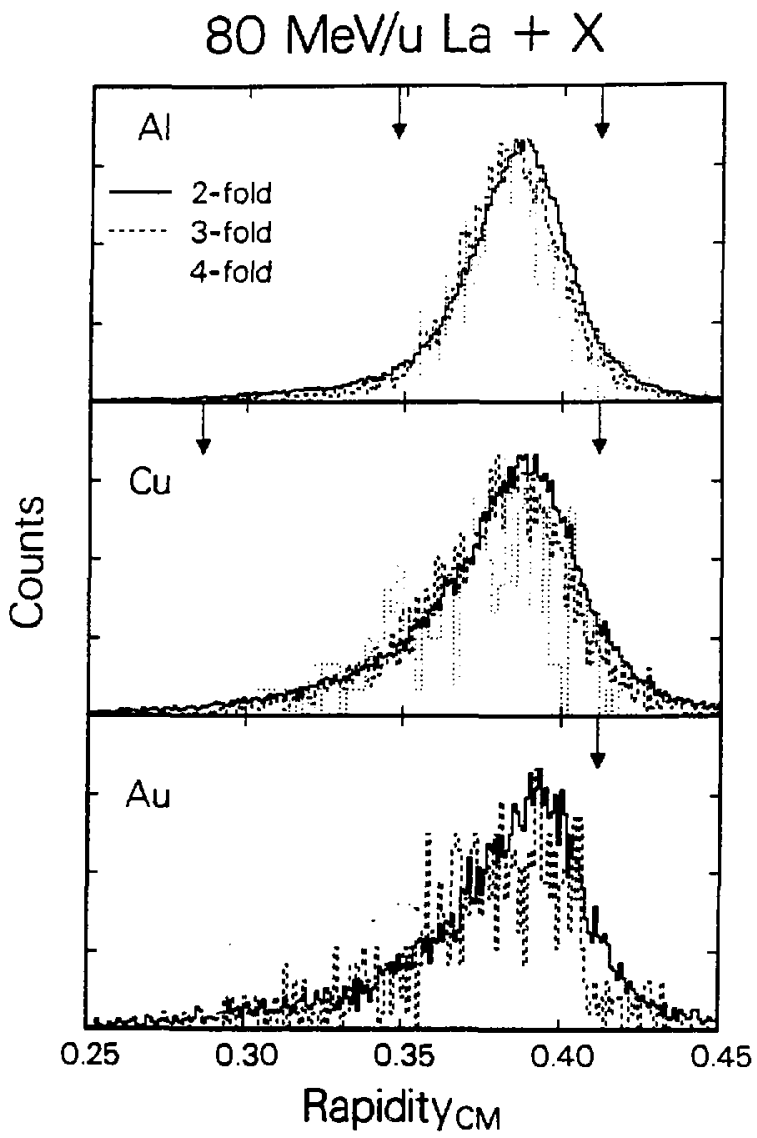

XBL $897-2762$

Figure IV.3 Distributions of the center-of-mass rapidity for 2-fold, 3-fold, and 4-fold complex fragment $(Z>2)$ events in the $80 \mathrm{MeV} / \mathrm{u}{ }^{139} \mathrm{La}+{ }^{27} \mathrm{Al}$, nat $\mathrm{Cu}$ and ${ }^{197} \mathrm{Au}$ reactions. The maxima of the distributions have been normalized to each other. The arrow at the larger rapidity in each subplot corresponds to the beam rapidity, the arrow at the smaller rapidity corresponds to the center-of-mass rapidity of the entrance channel. 


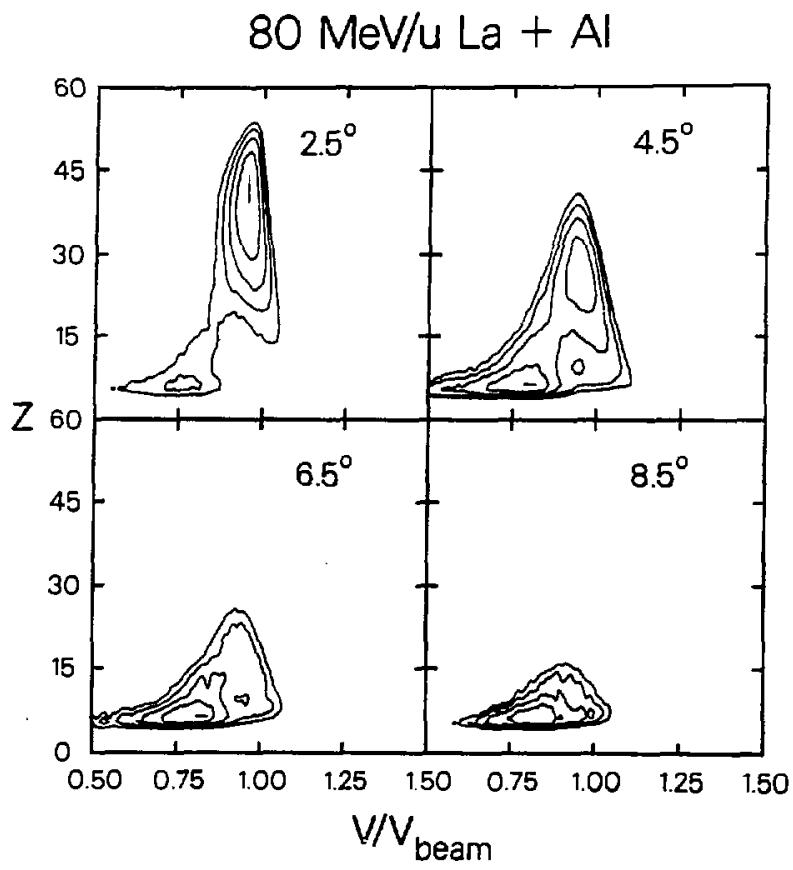

XEL $897-2759$

Figure IV.4 Logarithmic contours of the Lorent2-invariant cross section, $\frac{d^{2} \sigma}{\gamma^{4} v^{2} d \Omega d v}$, in the $Z$ - velocity plane for the $80 \mathrm{MeV} / \mathrm{u}^{139} \mathrm{La}+{ }^{27} \mathrm{Al}$ reaction at laboratory angles of $2.5^{\circ}$, $4.5^{\circ}, 6.5^{\circ}$, and 8.5. Neigh at $2.5^{\circ}$, a factor of 3.9 at $4.5^{\circ}$, a factor of 3.3 at $6.5^{\circ}$, and a factor of 3 at $8.5^{\circ}$. 


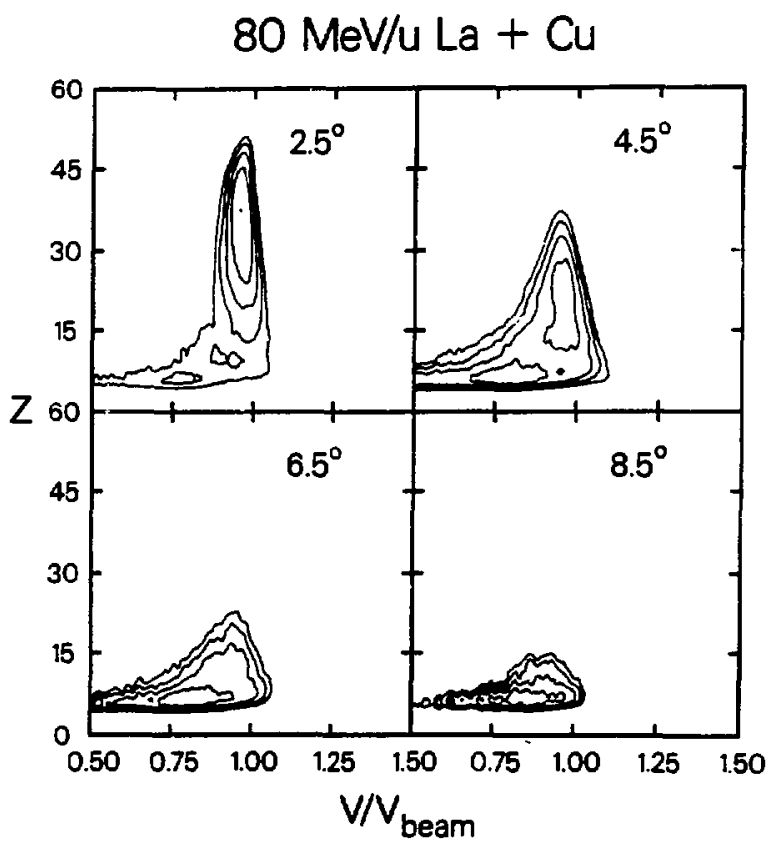

XE1 $897-2760$

Figure IV.5 Logarithmic contours of the Lorentz-invariant cross section, $\frac{d^{2} \sigma}{\gamma^{4} v^{2} d \Omega d v}$, in the $\mathrm{Z}$ - velocity plane for the $80 \mathrm{MeV} / \mathrm{u}{ }^{139} \mathrm{La}+$ nat Cu reaction at laboratory angles of $2.5^{\circ}$, $4.5^{\circ}, 6.5^{\circ}$, and $8.5^{\circ}$. Neighboring contours differ in value by approximately a factor of 3.6 at $2.5^{\circ}$, a fac ior of 3.4 at $4.5^{\circ}$, a factor of 3 at $6.5^{\circ}$, and a factor of 2.6 at $8.5^{\circ}$. 


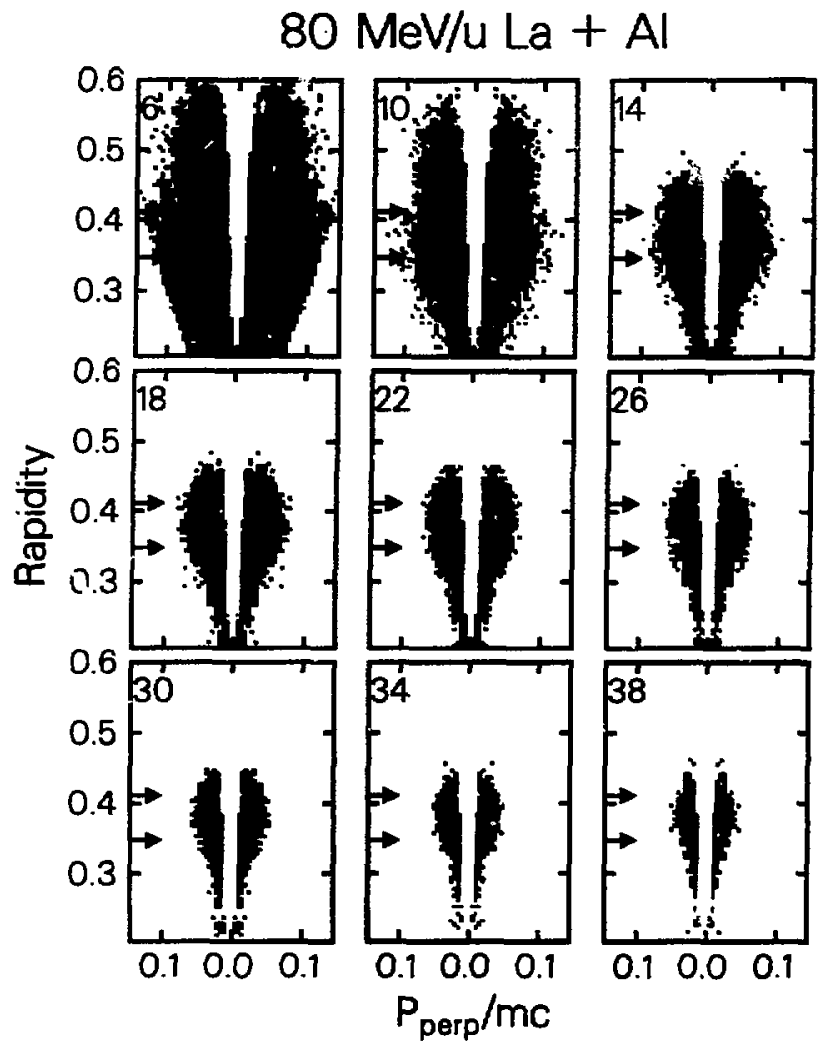

Figure IV.6 Linear density plots of $\frac{\partial^{2} \sigma}{\partial \mathrm{Y} \partial\left(\mathrm{F}_{\perp} / \mathrm{mc}\right)}$ in the rapidity - perpendicular momentum plane for representative $\mathrm{Z}$-values between 6 and 38 in the $80 \mathrm{MeV} / \mathrm{u}{ }^{139} \mathrm{La}+$ ${ }^{27} \mathrm{Al}$ reaction. The fragment $\mathrm{Z}$-values are indicated in the upper left hand comer of each subplot. The upper arrow in each subplot corresponds to the beam rapidity and the lower arrow to the center-of-mass rapidity of the entrance channel. Red indicates the regions of highest intensity. 


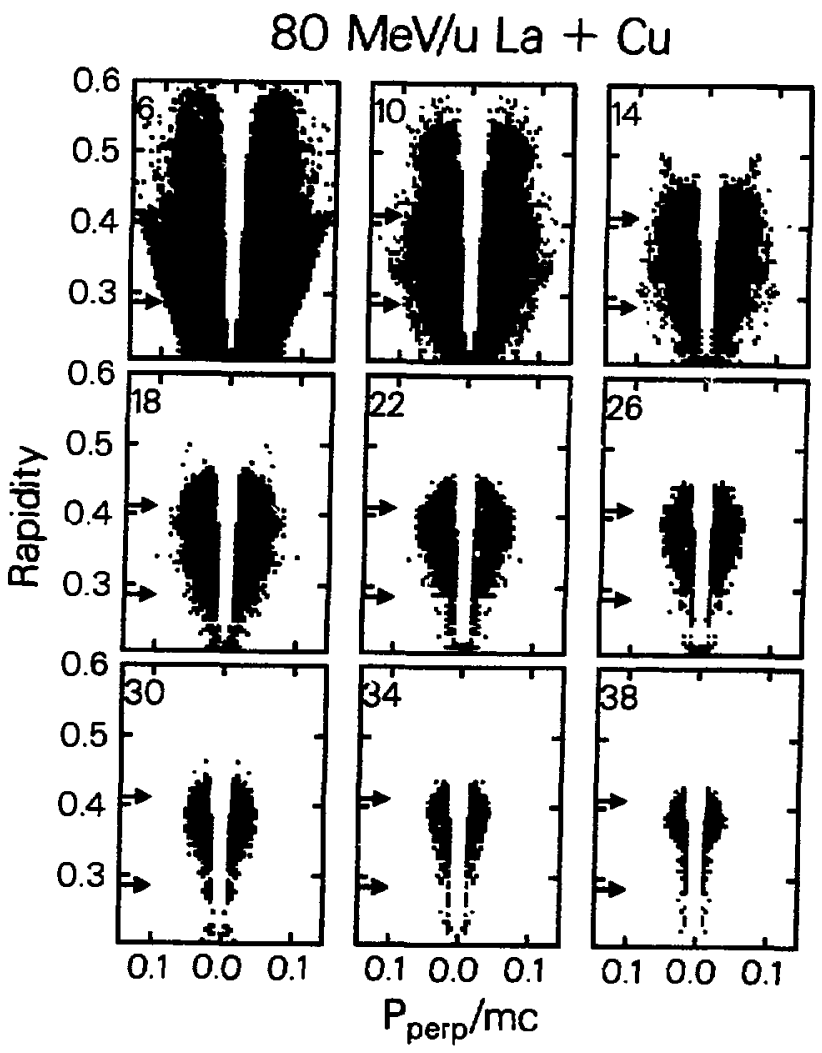

Figure IV.7 Linear density plots of $\frac{\partial^{2} \sigma}{\partial \mathrm{Y} \partial\left(\mathrm{P}_{\perp} / \mathrm{mc}\right)}$ in the rapidity - perpendicular momentum plane for representative $\mathrm{Z}$-values between 6 and 38 in the $80 \mathrm{MeV} / \mathrm{u}^{139} \mathrm{La}+$ nat $\mathrm{Cu}$ reaction. The fragment $Z$-values are indicated in the upper left hand comer of each subplot. The upper arrow in each subplot corresponds to the beam rapidity and the lower arrow to the center-of-mass rapidity of the entrance channel. Red indicates the regions of highest intensity. 


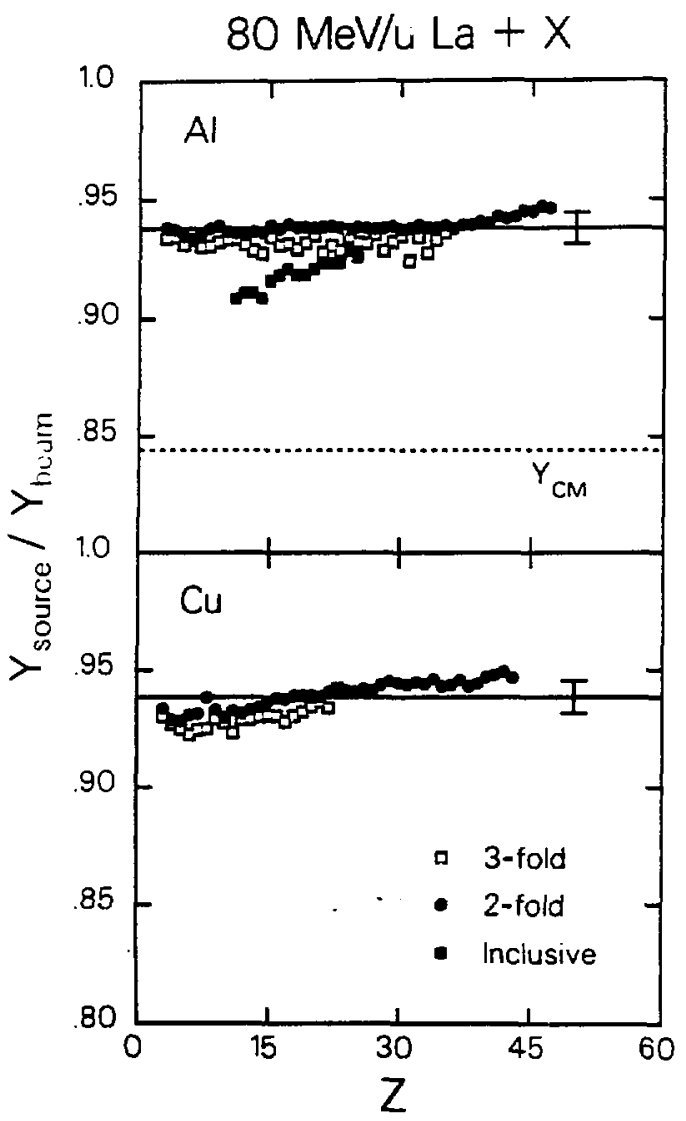

$X B L 897-2752$

Figure IV.8 Ratios of the source rapidity to the beam rapidity extracted from inclusive, 2-fold, and 3-fold events as a function of $\mathrm{Z}$-value for the $80 \mathrm{MeV} / \mathrm{u}{ }^{139} \mathrm{La}+{ }^{27} \mathrm{Al}$ and ${ }^{\text {nat }} \mathrm{Cu}$ reactions. The statistical errors of each point are smaller than the data points. The solid lines are the mean source rapidities from the 2-fold coincidences, the large error bars associated with these lines indicate the possible systematic error from the energy calibration and mass parametrization. The dashed line corresponds to the center-of-mass rapidity of the entrance channel. 


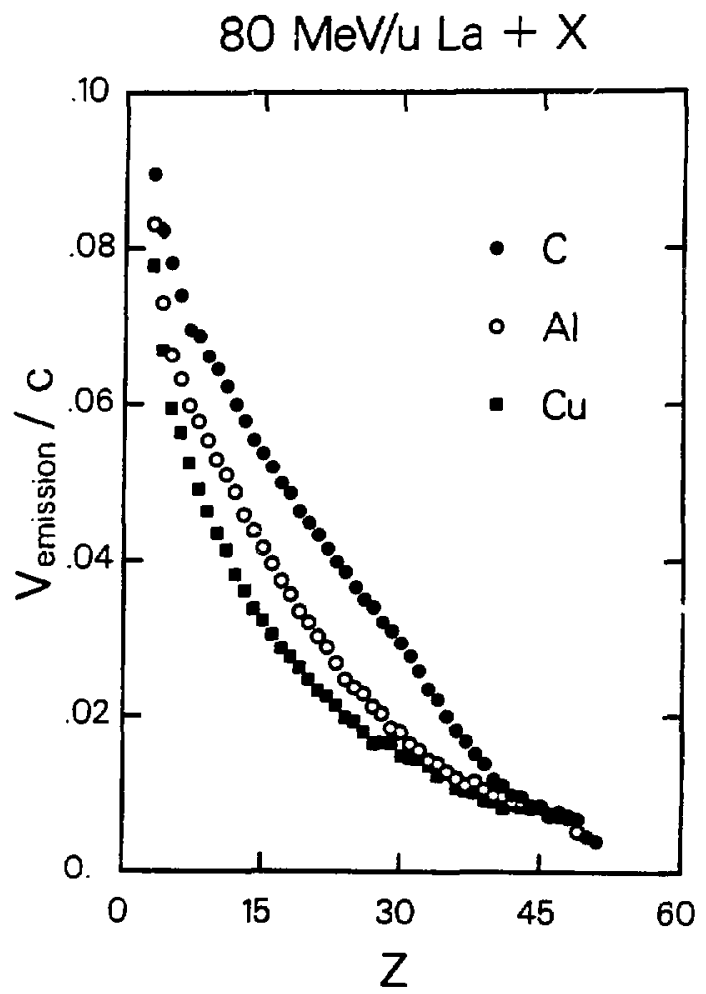

XBL 897-2764

Figure IV.9 The emission velocities as a function of Z-value from inclusive, 2-fold, and 3-fold events for the $80 \mathrm{MeV} / \mathrm{u}{ }^{139} \mathrm{La}+{ }^{27} \mathrm{Al}$ and nai $\mathrm{Cu}$ reactions. Error bars are shown for those data points in which the statistical errors are larger than the points. 


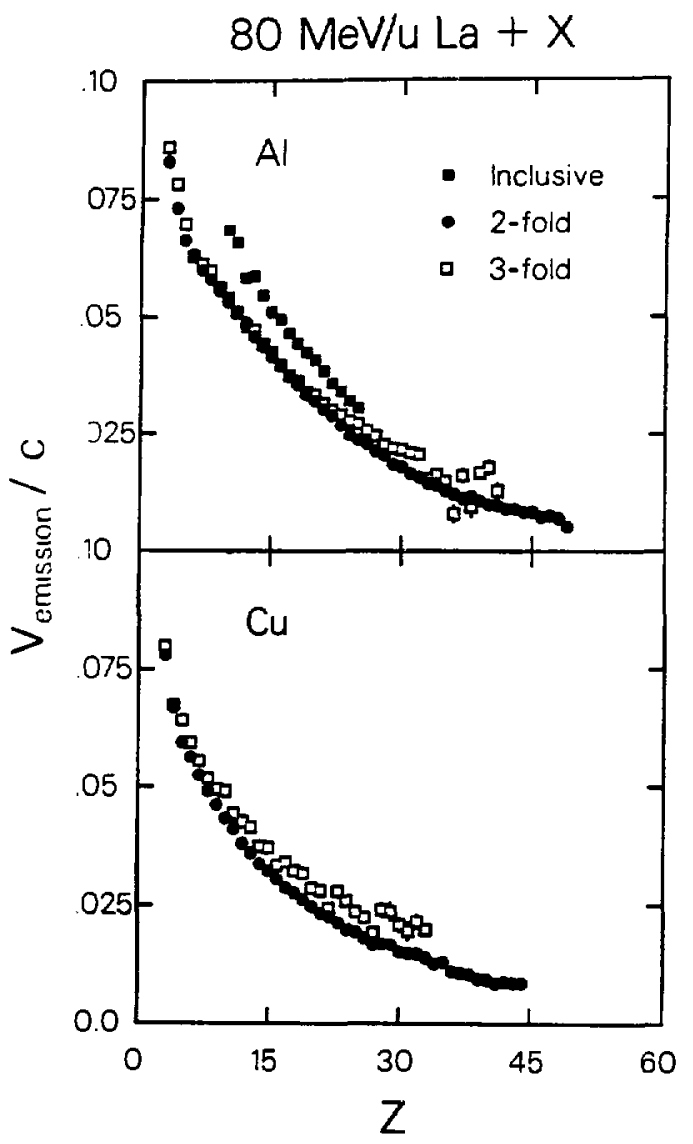

$X 9 L \quad 897-2763$

Figure IV.10 The emission velocities as a function of Z-value extracted from the 2-fold coincidence events for the $80 \mathrm{MeV} / \mathrm{u}{ }^{139} \mathrm{La}+{ }^{12} \mathrm{C},{ }^{27} \mathrm{Al}$, and nat $\mathrm{Cu}$ reactions. In all cases the statistical errors are smaller than the data points. 


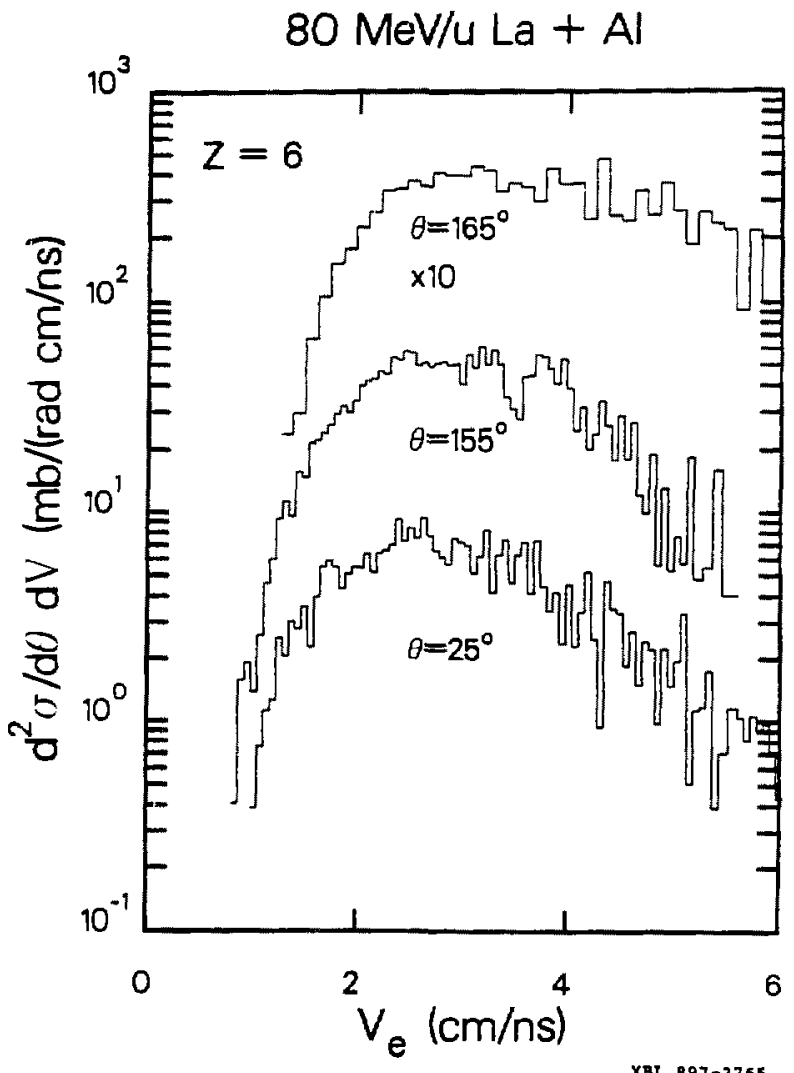

Figure IV.11 Emission velocity distributions for $Z=6$ fragments in the $80 \mathrm{MeV} / \mathrm{u}{ }^{139} \mathrm{La}$ $+{ }^{27} \mathrm{Al}$ reaction at angles of $25^{\circ}, 155^{\circ}$, and $165^{\circ}$ in the source frame. The distribution at $165^{\circ}$ has been multiplied by a factor of 10 . 


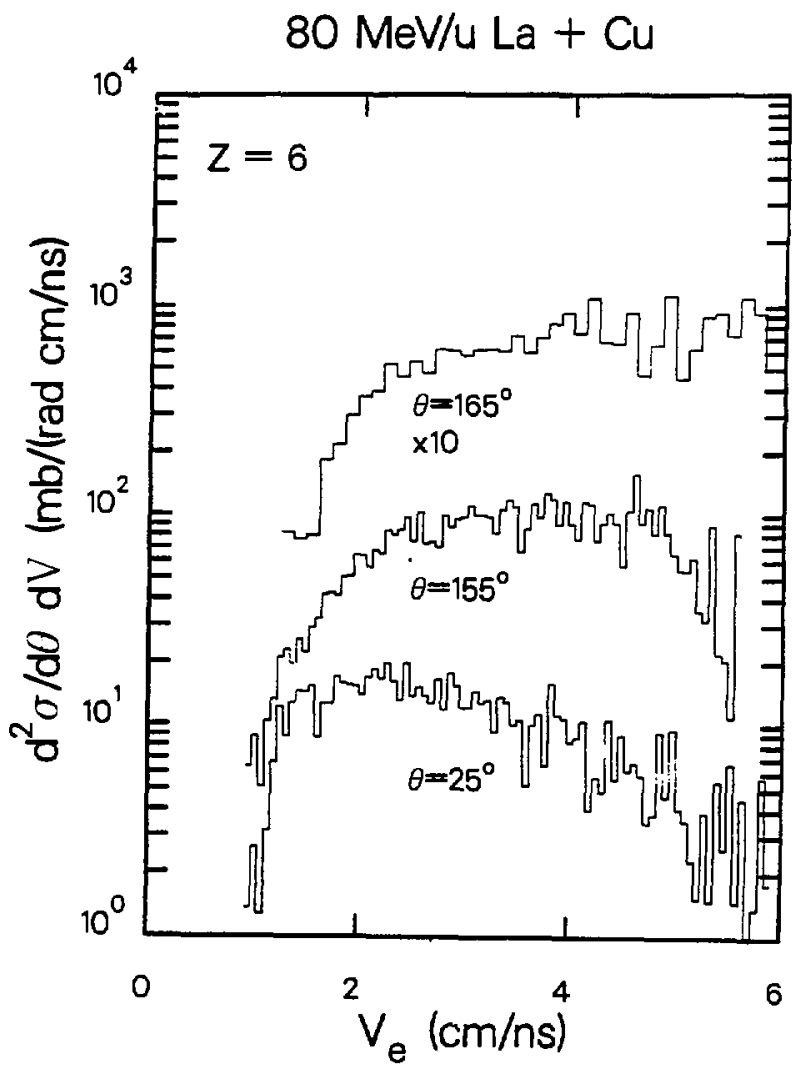

XBL $997-2766$

Figure IV.12 Emission velocity distributions for $Z=6$ fragments in the $80 \mathrm{MeV} / \mathrm{u}^{139} \mathrm{La}$ + nar $\mathrm{Cu}$ reaction at angles of $25^{\circ}, 155^{\circ}$, and $165^{\circ}$ in the source frame. The distribution at $165^{\circ}$ has been multiplied by a factor of 10 . 


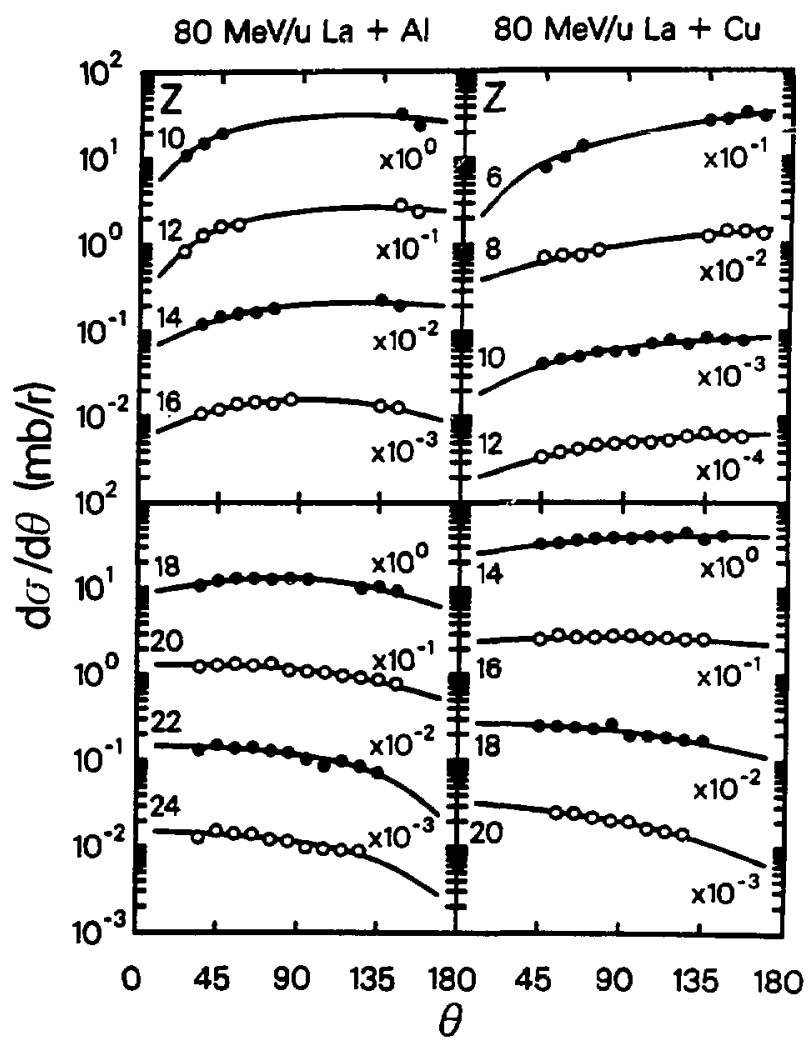

XBL $897-2767$

Figure IV.13 Representative source frame $d \sigma / d \theta$ angular distributions for the $80 \mathrm{MeV} / \mathrm{u}$ ${ }^{139} \mathrm{La}+{ }^{27} \mathrm{Al}$ and ${ }^{n a t} \mathrm{Cu}$ reactions. The $\mathrm{Z}$-value and normalization are indicated for each set of data. The solid lines show the fits to the distributions used to extract the absolute cross sections. At emission angles near $90^{\circ}$, the lighter complex fragments are emitted to larger laboratory angles than covered by the detector array, so the differential cross sections could not be measured. 


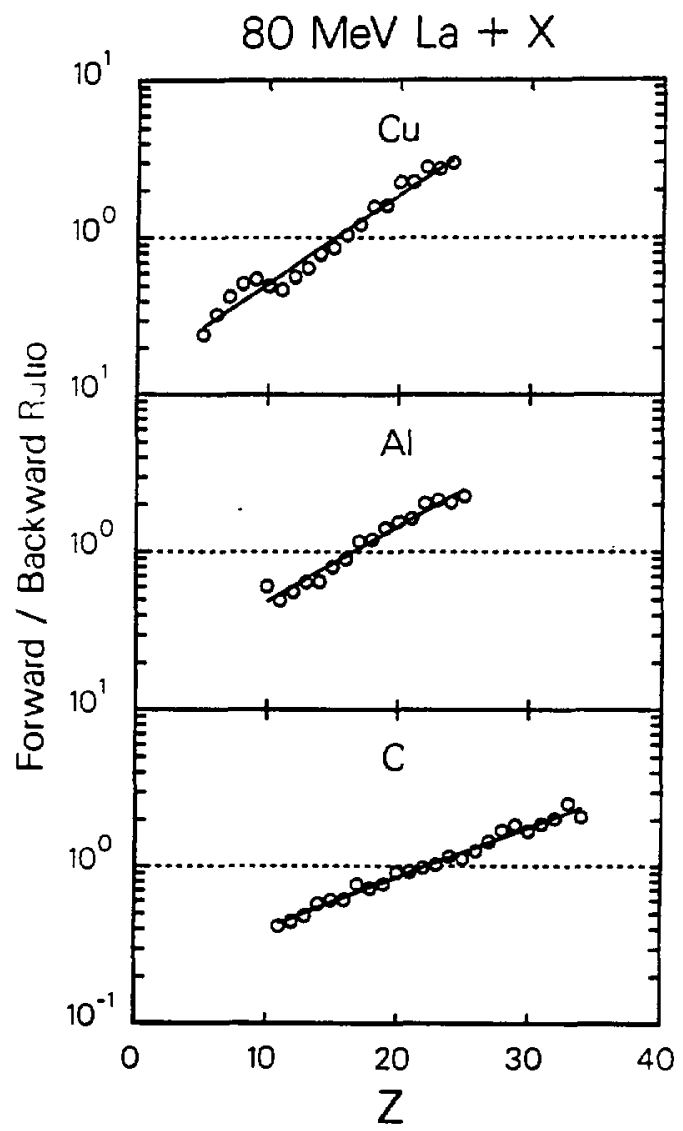

XBL $897-2768$

Figure IV.14 The ratios of the fragment yields forward of $90^{\circ}$ to those backward of $90^{\circ}$ (in the source frame) as a function of fragment $\mathrm{Z}$-value in the $80 \mathrm{MeV} / \mathrm{u}{ }^{139} \mathrm{La}+{ }^{12} \mathrm{C},{ }^{27} \mathrm{Al}$, and ${ }^{n a t} \mathrm{Cu}$ reactions. The solid lines are linear least squares fits to the $\log$ of the ratios. The dashed line $(=1)$ indicates forward/backward angular symmetry. 


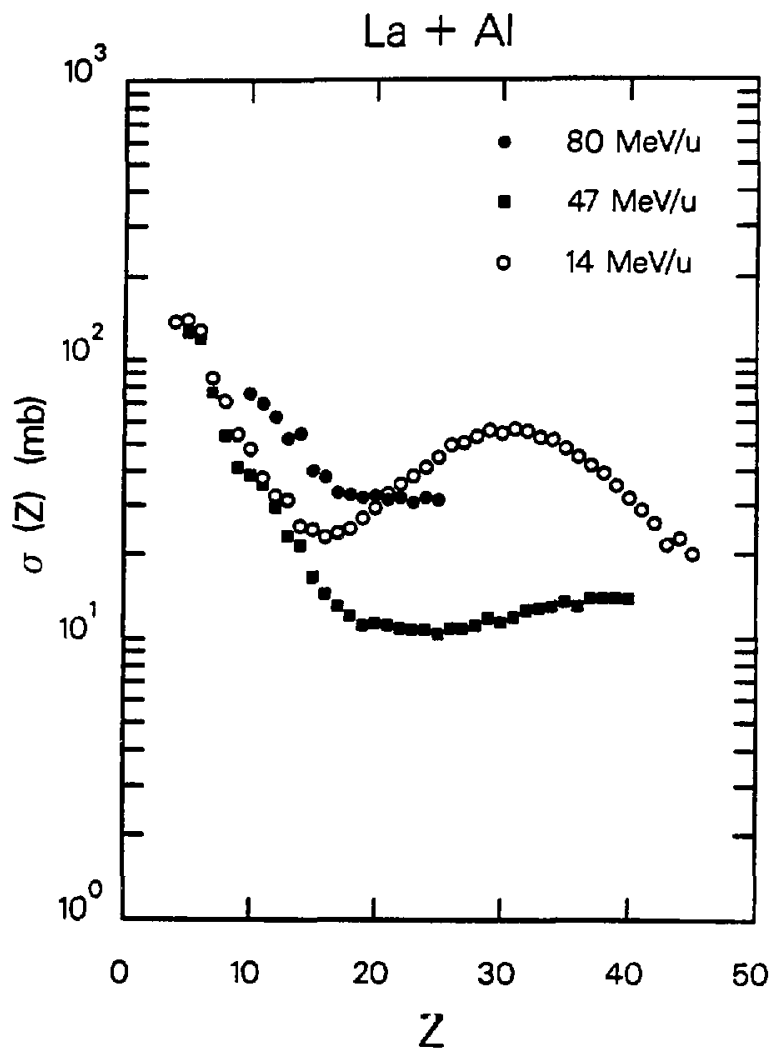

XEL $097-2769$

Figure IV.15 Angle-integrated complex fragment cross sections in the $14 \mathrm{MeV} / \mathrm{u}$ [Ch 89], $47 \mathrm{MeV} / \mathrm{u}$ [Ke 89], and $80 \mathrm{MeV} / \mathrm{u}{ }^{139} \mathrm{La}+{ }^{27} \mathrm{Al}$ reartions. The possible systematic errors associated with the absolute beam normalization, target thickness, and the integration procedure are approximately $20 \%$ at $14 \mathrm{MeV} / \mathrm{u}, 50 \%$ at $47 \mathrm{MeV} / \mathrm{u}$, and $20 \%$ at 80 MeV/u. 


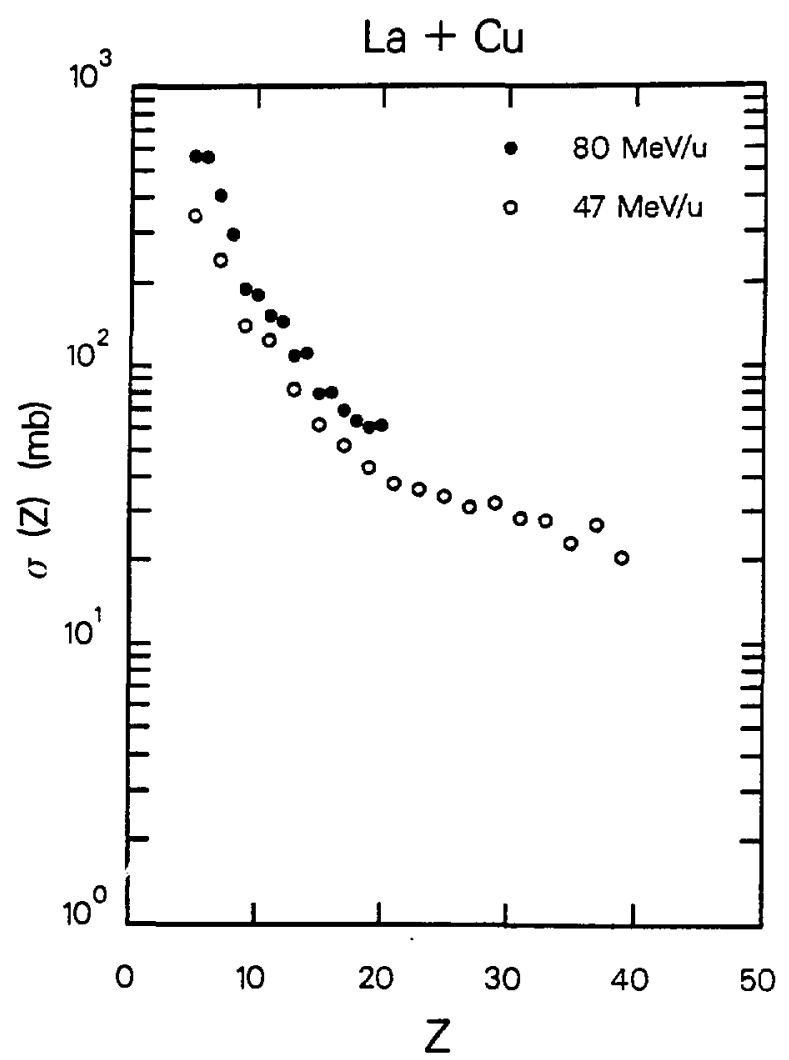

XBL $897-2770$

Figure IV.16 Angle-integrated cross sections of products in 47 [Ke 89] and $80 \mathrm{MeV} / \mathrm{u}$ ${ }^{139} \mathrm{La}+{ }^{\text {mat }} \mathrm{Cu}$ reactions. The possible systematic errors associated with the absolute beam normalization, target thickness, and the integration procedure are approximately $100 \%$ at $47 \mathrm{MeV} / \mathrm{u}$ and $20 \%$ at $80 \mathrm{MeV} / \mathrm{u}$. 


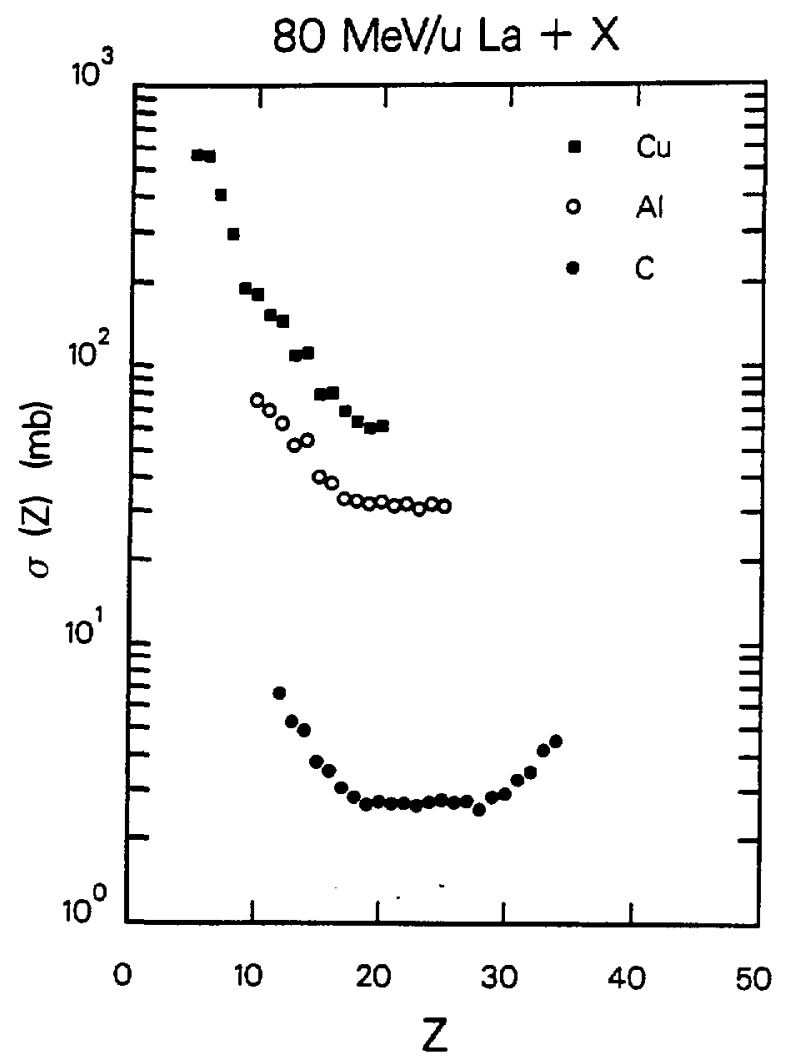

XBL $897-2771$

Figure IV.17 Angle-integrated cross sections of products in $80 \mathrm{MeV} / \mathrm{u}{ }^{139} \mathrm{La}+{ }^{12} \mathrm{C},{ }^{27} \mathrm{Al}$, and ${ }^{n a r} \mathrm{Cu}$ reactions. The possible systematic errors associated with the absolute beam normalization, target thickness, and the integration procedure are approximately $20 \%$. 


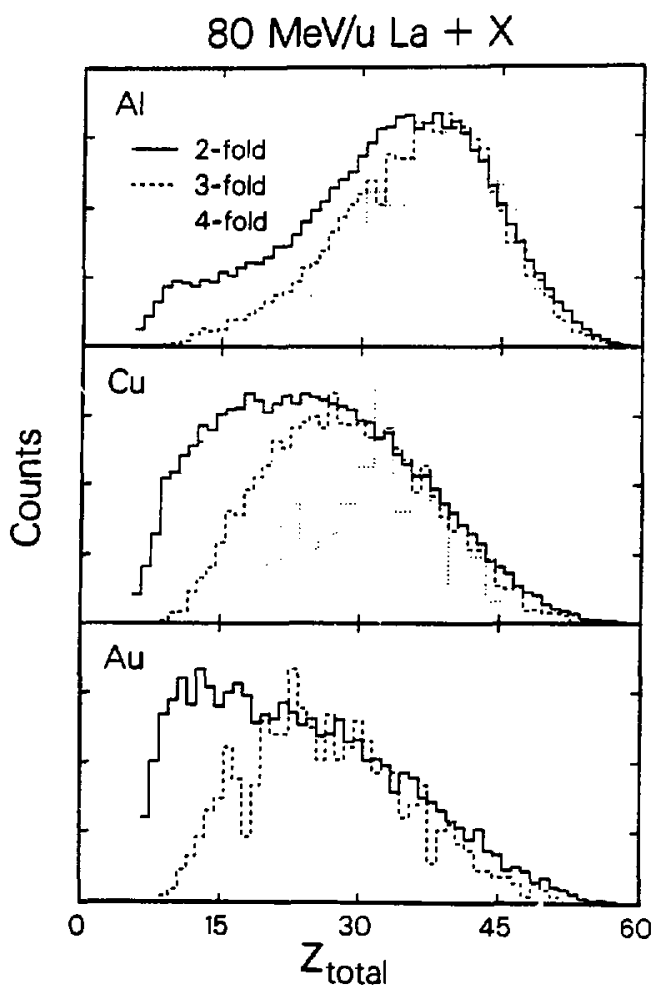

XBL $397-2772$

Figure IV.18 Distributions of $\mathrm{Z}_{\text {Total }}\left(\mathrm{Z}_{1}+\mathrm{Z}_{2}\right)$ for 2-fold, 3-fold, and 4-fold complex fragment $(\mathrm{Z}>2)$ events in the $80 \mathrm{MeV} / \mathrm{u}{ }^{139} \mathrm{La}+{ }^{12} \mathrm{C},{ }^{27} \mathrm{Al}$, and ritil $\mathrm{Cu}$ reactions. The maxima of the distributions have been normalized to each other. 


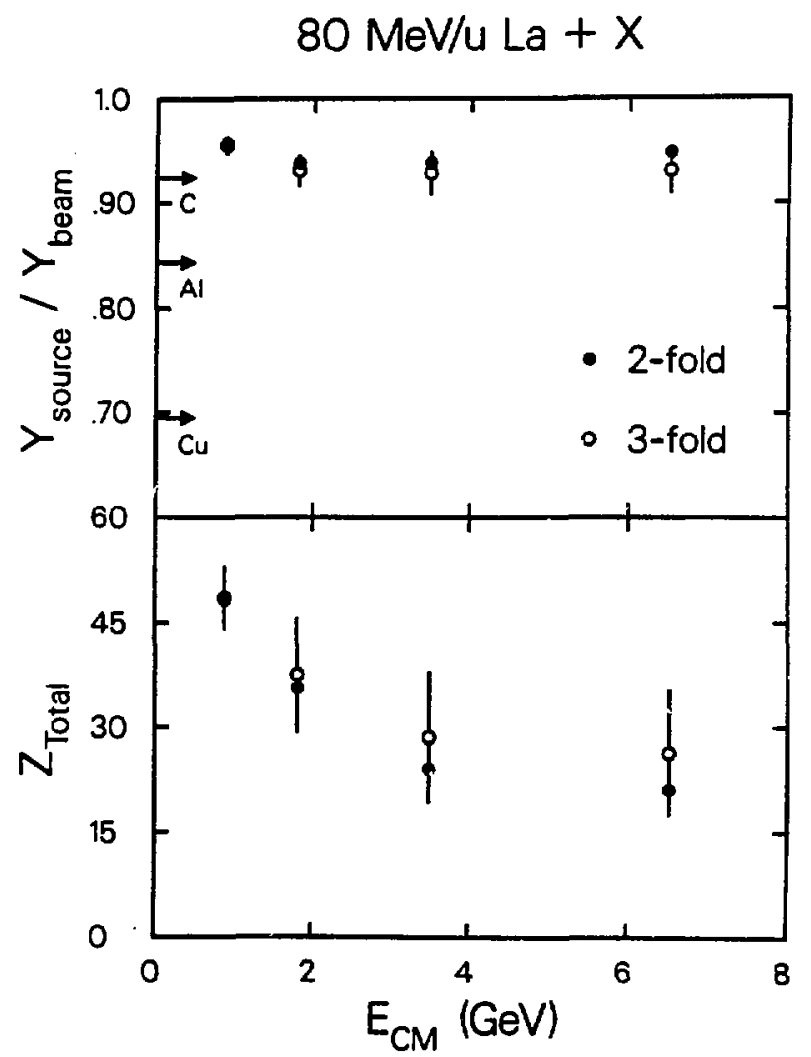

$\operatorname{XBL} 897-2773$

Figure IV.19 The upper portion of the Figure shows the average ratio of the source rapidity to the beam rapidity as a function of center-of-mass energy for $80 \mathrm{MeV} / \mathrm{u}{ }^{139} \mathrm{La}$ induced reactions. The widths of the rapidity distributions are indicated by the bars on the 3-fold data points. The entrance channel center-of-mass rapidities in the $80 \mathrm{MeV} / \mathrm{u}{ }^{139} \mathrm{La}+$ ${ }^{12} \mathrm{C},{ }^{27} \mathrm{Al}$, and ${ }^{n a} \mathrm{Cu}$ reactions are shown by the left arrows. The lower portion of the Figure shows the centroids of the total detected charge distributions as a function of centerof-mass energy for $80 \mathrm{MeV} / \mathrm{u}$ La-induced reactions. The widths of the total charge distributions are indicated by the bars on the 3-fold data points. 


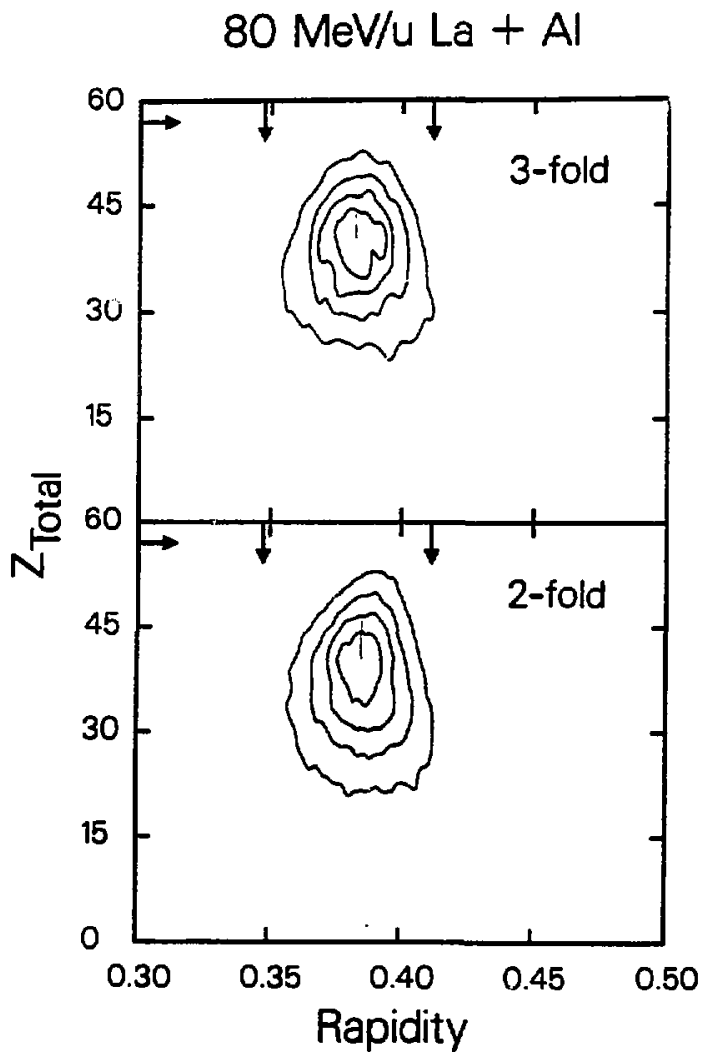

XBL $197-2774$

Figure IV.20 Linear contour plots of the total detected charge versus the rapidity of the center-of-mass for 2-fold and 3-fold coincidence events in the $80 \mathrm{MeV} / \mathrm{u}{ }^{139} \mathrm{La}+{ }^{27} \mathrm{Al}$ reaction. The sets of four lines correspond to contours with relative ratios of 4:3:2:1. The arrow along the ordinate indicates the charge of the ${ }^{139} \mathrm{La}$ projectile (57). The arrows at larger rapidity correspond to the beam rapidity, the arrows at smaller rapidity currespond to the center-of-mass rapidity of the enurance channel. 


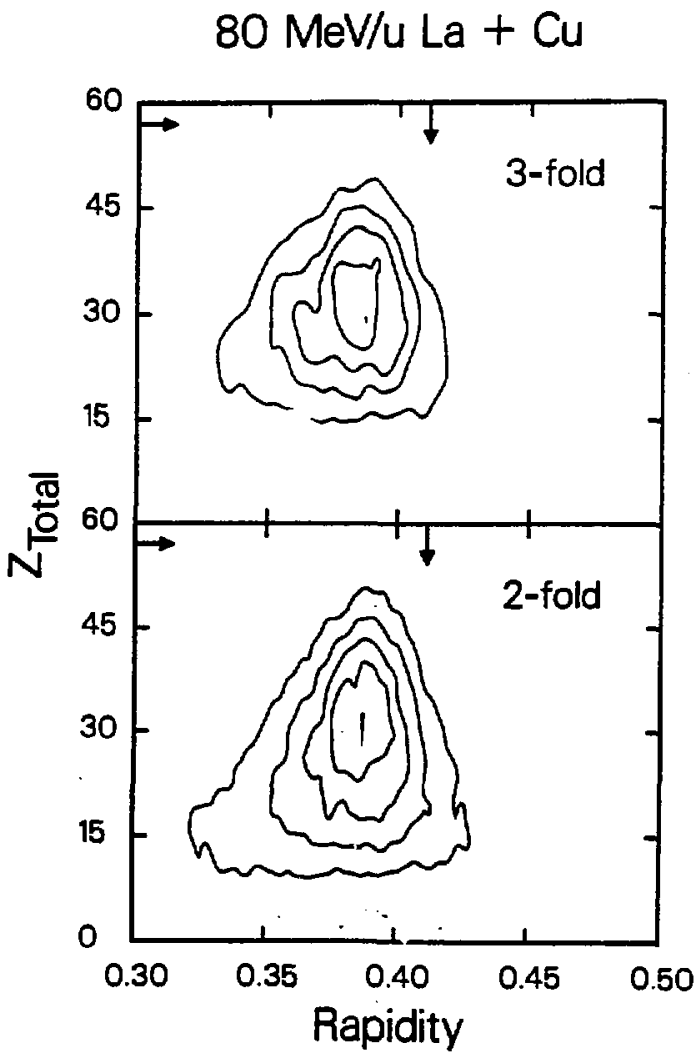

x.2 $997-2775$

Figure IV.21 Linear contour plots of the total detected charge versus the rapidity of the center-of-mass for 2-fold and 3-fold coincidence events in the $80 \mathrm{MeV} / \mathrm{u}^{139} \mathrm{La}+{ }^{\text {nar }} \mathrm{Cu}$ repaction. The sets of four lines correspond to contours with relative ratios of $4: 3: 2: 1$. The arrow along the ordinate indicates the charge of the La projectile (57). The arrow along the abscissa indicates the beam rapidity. The center-of-mass rapidity of the entrance channel = 0.2859 . 


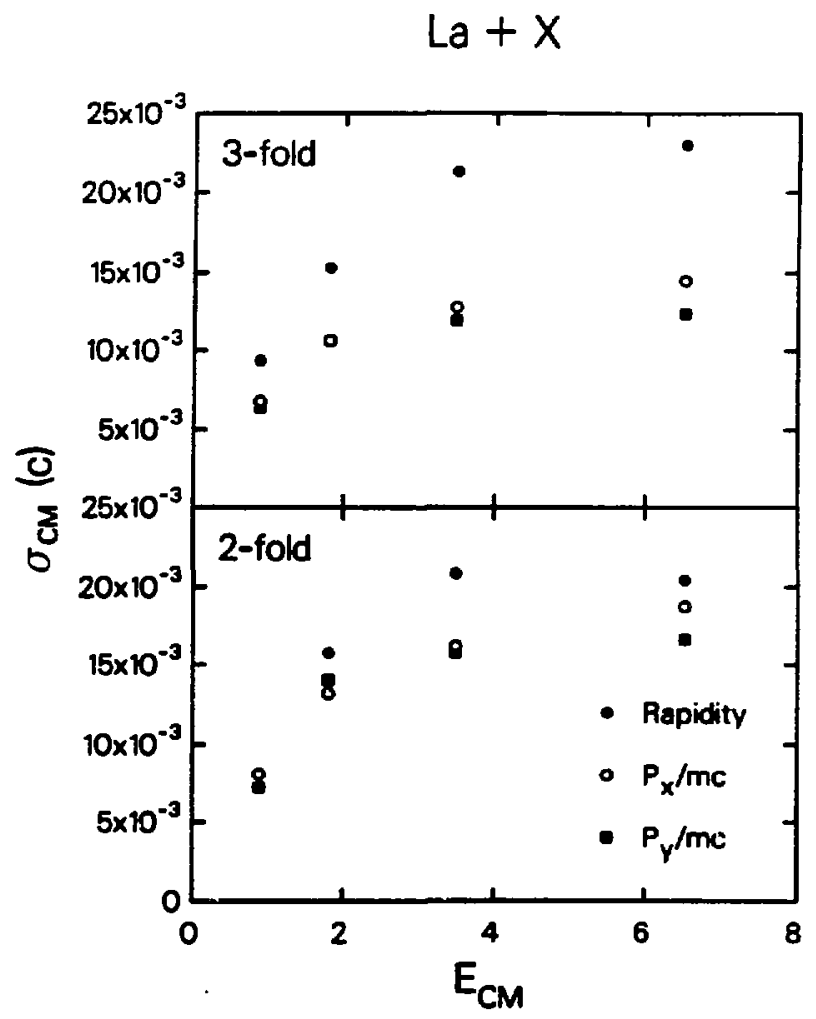

XBL $998-3049$

Figure IV.22 Widths of the 2-fold and 3-fold rapidity and momentum distributions as a function of the center-of-mass energy in the $80 \mathrm{MeV} / \mathrm{u}{ }^{139} \mathrm{La}+{ }^{27} \mathrm{Al}$, nat $\mathrm{Cu}$, and ${ }^{197} \mathrm{Au}$ reactions. 


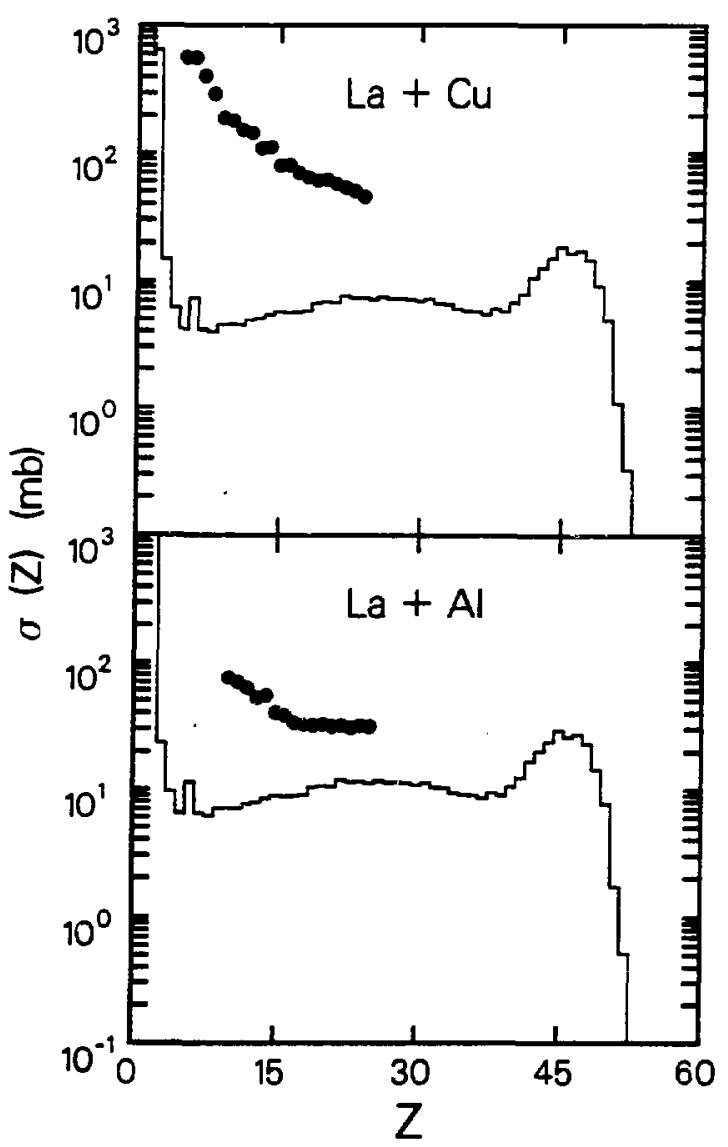

XBL $997-2878$

Figure IV.23 Experimental complex fragment cross sections (solid points) compared to statistical model calculations (histogram) using GEMINI (see text) for the $80 \mathrm{MeV} / \mathrm{L}{ }^{139} \mathrm{La}$ $+{ }^{27} \mathrm{Al}$ and nar $\mathrm{Cu}$ reactions. 


\section{SUMMARY AND CONCLUSIONS}

The incomplete fusion - compound statistical decay model has described the results of asymmetric reactions at bombarding energies $\leq 40 \mathrm{MeV} / \mathrm{u}$ very successfully. The goal of this thesis was to test this model at larger bombarding energies. In this section we will summarize our results and present the conclusions of the thesis. We will first discuss the $50 \mathrm{MeV} / \mathrm{u}^{139} \mathrm{La}+{ }^{12} \mathrm{C}$ reaction, followed by the 80 and $100 \mathrm{MeV} / \mathrm{u}{ }^{139} \mathrm{La}+{ }^{12} \mathrm{C}$ reactions, and finally the $80 \mathrm{MeV} / \mathrm{u}{ }^{139} \mathrm{La}+{ }^{27} \mathrm{Al}$, nat $\mathrm{Cu}$, and ${ }^{197} \mathrm{Au}$ reactions.

\section{A. $50 \mathrm{MeV} / \mathrm{L}^{139} \mathrm{La}+{ }^{12} \mathrm{C}$}

In this reaction, we have shown that following incomplete fusion, the complex fragments are produced in highly equilibrated binary processes. Incomplete fusion is indicated by the source rapidity distribution, which is very sharp and corresponds to the ${ }^{139} \mathrm{La}$ projectile capturing approximately one-half of the ${ }^{12} \mathrm{C}$ target. The experimental source rapidities are independent of Z-value within the range of $21 \leq Z \leq 35$, showing the common origin of all of the complex fragments.

The binary nature of the decay process is illustrated by the 2 -fold coincidence events $\left(Z_{1}+Z_{2}\right)$, which sum to a nearly constant value of total charge, by the $Z$ versus velocity contour plots, which show Coulomb ridges characteristic of relaxed binary decay, and by the well-defined Coulomb rings in the $\frac{\partial^{2} \sigma}{\partial v_{\perp} \partial v_{\|}}$density plots. Additional evidence for the binary nature of the mechanism is given by the coincidence efficiency (coincidence/singles ratio) for a given Z-value, which is in agreement with a Monte Carlo simulation of binary decay.

The relaxed nature of the decay process is shown by the source frame emission velocities and angular distributions. The fragment emission velocities show excellent agreement with the predictions of completely relaxed (Coulombic) binary decay. The forward/backward symmetry of all of the fragment angular distributions demonstrates the 
complete relaxation of the angular degrees of freedom.

We have used the average experimental source rapidity to infer the mass, charge, excitation energy, and angular momentum distribution of the incomplete fusion product. To determine conclusively whether the complex fragments are produced by compound nucleus decay it is necessary tu compare the absolute cross sections with predictions from the compound statistical decay model [Mo 72, Mo 75]. A statistical decay code (GEMENI [Ch 88b]) based on this model, which has had great success in reproducing the complex fragment cross sections following complete fusion reactions at lower bombarding energies [Ch 88b, $\mathrm{Ha} \mathrm{89,} \mathrm{Ch} \mathrm{89],} \mathrm{is} \mathrm{also} \mathrm{able} \mathrm{to} \mathrm{reproduce} \mathrm{the} \mathrm{experimental} \mathrm{cross} \mathrm{sections} \mathrm{in} \mathrm{this}$ reaction. The excellent agreement between the statistical model calculations and the experimental data in the ${ }^{139} \mathrm{La}+{ }^{12} \mathrm{C}$ system from $14-50 \mathrm{MeV} / \mathrm{u}$ is very strong evidence for compound nucleus formation in all of these reactions.

B. 80 and $100 \mathrm{MeV} / \mathrm{L}^{139} \mathrm{La}+{ }^{12} \mathrm{C}$

In these reactions, we also have evidence for highly equilibrated binary decay processes following incomplete fusion. The source rapidities once again correspond to incomplete fusion processes in which approximately one-half of the ${ }^{12} \mathrm{C}$ target is transferred to the ${ }^{139} \mathrm{La}$ projectile. The source rapidity distributions are independent of $Z$ value and of the complex fragment multiplicity, showing the common origin of all of the complex fragments.

The binary nature of the process is illustrated by the peaks in the $\left(Z_{1}+Z_{2}\right)$ charge distributions, by the Coulomb ridges in the $Z$ versus velocity contour plots, by the welldefined elliptical distributions in the $\frac{\partial^{2} \sigma}{\partial Y \partial\left(P_{\perp} / \mathrm{mc}\right)}$ density plots, and by the symmetric charge distributions (at $80 \mathrm{MeV} / \mathrm{u}$ ). The fragment emission velocities are Coulombic and completely relaxed. However, except for the fragments from symmetric and nearly symmetric decay $(Z \sim 20-24)$, the source frame angular distributions are anisotropic and inconsistent with purely statistical emission. Similarly, while the symmetric cross sections 
can be explained as originating solely from compound statistical decay following incomplete fusion, the asymmetric yield is larger than predicted by the model.

We have speculated that the anisotropic angular distributions and the increase in the non-equilibrium yield may be associated with the threshold for the decoupling of the fireball.

C. $80 \mathrm{MeV} / \mathrm{u}^{139} \mathrm{La} \mathrm{a}^{27} \mathrm{Al}{ }^{\text {nat } \mathrm{Cu}}{ }^{197} \mathrm{Au}$

The majority of the products from these resctions cannot be explained using the incomplete fusion - compound statistical emission model. We have observed that the source rapidity no longer characterizes the reactions as at lower bombarding energy [Co 89a]. This contradicts the incomplete fusion picture and strongly suggests that incomplete fusion is not applicable in the ${ }^{139} \mathrm{La}+{ }^{n a 4} \mathrm{Cu}$ and ${ }^{139} \mathrm{La}+{ }^{197} \mathrm{Au}$ reactions. The source rapidities also show dependences upon the $Z$-value of the detected fragment indicating a range of sources for the complex fragments.

The centroids of the coincidence charge distributions decrease and their widths increase as the target mass increases. The disappearance of the characteristic binary signature suggests a dramatic increase in the light charged particle and complex fragment multiplicity in these reactions. The complex fragments are no longer emitted onto welldefined Coulomb rings as they are in the ${ }^{139} \mathrm{La}+{ }^{12} \mathrm{C}$ reactions. While it appears that the complex fragment multiplicity is usually no greater than two in the ${ }^{139} \mathrm{La}+{ }^{27} \mathrm{Al}$ reaction, the velocity distributions are considerably broader than those of the ${ }^{139} \mathrm{Lz}+{ }^{12} \mathrm{C}$ reactions, perhaps due to a significant increase in the light particle multiplicity and/or the broader range of source rapidities. For the ${ }^{139} \mathrm{La}+{ }^{n a t} \mathrm{Cu}$ reaction, all evidence points to final states with $>\mathbf{3}$ complex fragments on the average; there is no longer any hint of two-body kinematics. The observed decrease in the emission velocity for fragments of a given charge as the target mass is increased also suggests an increase in the light charged particle and complex fragment multiplicity.

The inclusive angular distributions in these reactions are not consistent with 
compound nucleus emission. They become less isotropic and change more rapidly from backward to forward peaked as the target mass is increased. Only fragments withir. a limited range of $Z$-values $(Z \sim 15)$ have isotropic angular distributions. For the other fragments, the fraction of the cross section that can be attributed to isotropic emission is very small. The cross sections calculated from statistical compound nucleus decay are much smaller than the experimental cross sections, even for those products with isotropic angular distributions.

Light complex fragments with intermediate rapidity have been associated with the deep-inelastic/non-equilibrium mechanism at bombarding energies $\leq 50 \mathrm{MeV} / \mathrm{u}$. We have previously observed, and commented on, the disappearance of these fragments in the 80 $\mathrm{MeV} / \mathrm{u}{ }^{139} \mathrm{La}+{ }^{12} \mathrm{C}$ reaction. In the reactions with the heavier targets, we again observe such fragments. In these reactions it is not clear whether these fragments are still associated with the low-energy deep-inelastic mechanism or whether they are related to the onset of the rireball, as suggested in the ${ }^{139} \mathrm{La}+{ }^{12} \mathrm{C}$ reaction.

We have only touched on the basic observables in these reactions with heavier targets. To get a better idea of the reaction mechanisms leading to these very complex final states it would be instructive to investigate these more symmetric reactions in the same way as the asymmetric were investigated: by a systematic study beginning in the deep-inelastic regime and tracking the reactions up gradually in energy. Work in this direction is already in progress [Co 89]. 


\section{APPENDIX I: DETECTOR EFFICIENCY AND KINEMATICS}

\section{A. Detector Efficiency}

In order to determine cross sections we must correct for the geometric efficiency of the detectors. The geometric efficiency at a given polar angle $\left(\theta_{\mathrm{lab}}\right)$ is equal to the azimuthal angle $\left[\Delta \phi\left(\theta_{1 \mathrm{ab}}\right)\right]$ subtended by the detectors divided by $2 \pi, \operatorname{Eff}\left(\theta_{\mathrm{lab}}\right)=\frac{\Delta \phi\left(\theta_{\mathrm{lab}}\right)}{2 \pi}$. At 50 $\mathrm{MeV} / \mathrm{u}$, the detectors had continuous position resolution, so the efficiency was obtained by performing a numerical integration over $\phi$ at each $\theta_{\text {lab }}$.

The geometric efficiency at the higher bombarding energies was calculated using a different method. Each of the 11 detector telescopes has $15 \times 15=225$ discrete position elements. The solid angle of an individual element is:

$$
\Delta \omega\left(\theta_{1 a b}\right) \equiv \frac{A \cos \vartheta}{R^{2}}
$$

where $A$ is the area of the position element, $\vartheta$ is the angle between the center of the telescope and the center of the element, and $R$ is the distance from the target to the first 5 $\mathrm{mm} \mathrm{Si}(\mathrm{Li})$ detectors $(\sim 924 \mathrm{~mm})$. By summing over the position elements of all of the telescopes, the solid angle as a function of laboratory angle, $\Delta \Omega\left(\theta_{\mathrm{lab}}\right)$, was calculated in steps of $0.1^{\circ}$. To determine the detector efficiency, we must calculate the total solid angle of a $0.1^{\circ}$ slice at a given laboratory angle. To very good accuracy, this solid angle is given by:

$$
\Omega\left(\theta_{12 b}\right) \equiv 2 \pi \sin \theta_{1 a b} 0.1^{*} \frac{\pi}{180^{\circ}}
$$

The resulting efficiency is then simply: 


$$
\operatorname{Eff}\left(\theta_{12 b}\right)=\frac{\Delta \Omega\left(\theta_{12 b}\right)}{\Omega\left(\theta_{1 a b}\right)}
$$

\section{B. Kinematics}

\section{1. $50 \mathrm{MeV} / \mathrm{L}{ }^{139} \mathrm{La}+{ }^{2} \mathrm{CC}$}

At all bombarding energies, the velocity of the fragments was calculated relativistically from the mass $(M)$ and the measured kinetic energy $\left(E_{k i n}\right)$ as:

$$
V=\sqrt{1-\left(\frac{M}{M+E_{k i n}}\right)^{2}}
$$

Here the velocity is expressed in units of the speed of light (c) and $\mathrm{M}=931.5 \mathrm{~A} \mathrm{MeV}$, where $A$ is the mass of the fragment calculated using the mass parametrizations given in the Experimental section. For convenience we have set $c=1$ in all of these equations.

The source velocities were calculated from the laboratory velocity distributions. Let us define $\mathrm{V}_{\mathrm{a}}$ as the laboratory velocity of a fragment of mass $\mathrm{A}$ emitted forward in the source frame and detected at a laboratory angle $\theta$, and $V_{b}$ as the velocity of a fragment of mass A emitted backward in the source frame and detected at the same angle (see Fig II.1). From simple geometrical arguments, the source velocity can be extracted (nonrelativistically) from the inclusive velocity distributions as:

$$
V_{\text {source }} \frac{V_{a}+V_{b}}{2 \cos \theta}
$$

In practice, since the detectors span a range of laboratory angles, the source velocities were extracted using the equation $V_{s} \cong \frac{1}{2}\left(X_{a}+X_{b}\right)$, where $X_{a}$ and $X_{b}$ are the centroid of the $\frac{V}{\cos \theta}$ distributions. Simulations have shown that this approximation is good to better than $0.4 \%$ for determining the $V_{\text {source }}$ from equation (A4) above [Ch 88a]. 
The use of the non-relativistic kinematics is expected to introduce less than a $0.3 \%$ error in the source velocity determination.

From the radii of the distributions in velocity space we can determine the average emission velocity for each Z-value. At $50 \mathrm{MeV} / \mathrm{u}$, the lab distributions were explicitly transformed event by event into the source frame determined from the above analysis using the non-relativistic (Galilean) transformation:

$$
\begin{aligned}
& v_{\|}=v \cos \theta \\
& v_{\perp}=v \sin \theta \\
& v^{\prime}=\sqrt{\left(v_{\|}-v_{\text {source }}\right)^{2}+v_{\perp}^{2}} \\
& \theta^{\prime}=\tan ^{-1} \frac{v_{\perp}}{v_{\| 1}-v_{\text {source }}} .
\end{aligned}
$$

The use of the non-relativistic transformation at $50 \mathrm{MeV} / \mathrm{u}$ introduces errors of the order of $\gamma(5 \%)$ in the emission velocities 5 .

2. 80 and $100 \mathrm{MeV} / \mathrm{u}{ }^{139} \mathrm{La}+{ }^{12} \mathrm{C},{ }^{27} \mathrm{Al}$, nat $\mathrm{Cu}$, and ${ }^{197} \mathrm{Au}$

From the measured $Z$-values at the higher bombarding energies, the fragment masses were calculated from the mass parametrization given in the Experimental section. Then from the mass $(M$, defined as above), the laboratory detection angle ( $\theta$ ), and the measured kinetic energy $\left(E_{k i n}\right)$, the laboratory momentum $(P)$, longitudinal velocity $\left(v_{\|}\right)$, and rapidity $(Y)$ were determined:

$$
P=\sqrt{\left(E_{k i n}+M\right)^{2}-M^{2}}
$$




$$
P_{\|}=P \cos \theta
$$

$$
P_{\perp}=P \sin \theta
$$

$$
v_{\|}=\frac{P_{\|}}{E_{\text {kin }}+M}
$$

$$
Y=\tanh ^{-1}\left(v_{\|}\right)=0.5 \ln \frac{E_{k i n}+M+P_{\|}}{E_{k i n}+M-P_{\|}} .
$$

Rapidity is used as a variable in relativistic kinematics because it has the convenient property that its Lorentz-transformation is linear:

$$
\mathbf{Y}^{\prime}=\mathbf{Y}_{0}+\mathbf{Y}
$$

In this equation $Y_{0}$ is the rapidity in the unprimed frame (i.e. source frame), $Y$ is the rapidity of the primed frame with respect to the unprimed frame, and $Y^{\prime}$ is the resulting rapidity in the primed frame (i.e. lab frame). Rapidity is not a vector and is defined along only one direction, usually that of the beam.

In order to extract the source rapidities from the inclusive events at 80 and 100 $\mathrm{MeV} / \mathrm{u}$, the experimental distributions were corrected for detector efficiency as described above and Lorentz-transformed into an assumed source frame:

$$
\begin{aligned}
& P_{\|}{ }^{\prime}=\cosh \left(Y^{\prime}\right) P_{\|}-\cosh \left(Y^{\prime}\right) \tanh \left(Y^{\prime}\right)\left(E_{k i n}+M\right) \\
& P_{\perp}{ }^{\prime}=P_{\perp} \\
& E_{k_{\text {in }}{ }^{\prime}}=\cosh \left(Y^{\prime}\right)\left(E_{k \text { kin }}+M\right)-\cosh \left(Y^{\prime}\right) \tanh \left(Y^{\prime}\right) P_{\|}
\end{aligned}
$$




$$
\begin{aligned}
& P^{\prime}=\sqrt{P_{\|^{\prime 2}+P_{\perp}{ }^{\prime 2}}} \\
& v^{\prime}=\frac{P^{\prime}}{E^{\prime}} \\
& \theta^{\prime}=\tan ^{-1}\left(P_{\perp}^{\prime} / P_{\|^{\prime}}\right) .
\end{aligned}
$$

Here $Y^{\prime}$ is the assumed rapidity of the source. The centroids of the emission velocity ( $\left.v^{\prime}\right)$ distributions were determined as a function of the emission angle ( $\left.\theta^{\prime \prime}\right)$

The distribution of centroids as a function of emission angle was then fit to an ellipse. The equation for an ellipse in polar coordinates is:

$$
v^{\prime}=\frac{-B+\sqrt{B^{2}-4 A C}}{2 A}
$$

with

$$
\begin{aligned}
& A=\left(\frac{\cos \theta^{\prime}}{v_{\|}}\right)^{2}+\left(\frac{\sin \theta^{\prime}}{v_{\perp}}\right)^{2} \\
& B=-2\left(\frac{h \cos \theta^{\prime}}{v_{\|}^{2}}\right) \\
& C=\frac{h^{2}}{v_{\| 1}^{2}}-1 .
\end{aligned}
$$

A least squares fit of $v^{\prime}$ and $\theta^{\prime}$ was performed and the following parameters extracted from the fits: $v_{\| l}$, the emission velocity parallel to the beam, $v_{1}$, the emission velocity perpendicular to the beam, and $h$, the correction to the assumed source rapidity $\left(Y^{\prime}\right)$. The 
center of the ellipses was assumed to lie along the beam vector. The measured source rapidities equal the assumed rapidity $\left(Y^{\prime}\right)$ plus the correction (h) extracted from the fitting procedure.

The source rapidity can also be obtained as the center-of-mass rapidity of the coincidence events. In any frame the velocity is given by equation (A20) above. With mare than one fragment, the center-of-mass velocity of the system of fragments is given by:

$$
v_{\|}=\frac{\sum_{i=1}^{n} P_{\| i}}{\sum_{i=1}^{n}\left(E_{k i n_{i}}+M_{i}\right)},
$$

where the summation is over all of the fragments. Since there was a clear peak in the coincidence charge distributions for the ${ }^{139} \mathrm{La}+{ }^{12} \mathrm{C}$ reactions, a gate was set upon the peak to exclude events in which one large fragment was missed. Since no wcll-defined peak was observed in the reactions with the heavier targets, no gate was set on the total detected charge.

To facilitate comparisons with the higher energy data, the measured source velocities in the $50 \mathrm{MeV} / \mathrm{u}{ }^{139} \mathrm{La}+{ }^{12} \mathrm{C}$ reaction (A4) were transformed into rapidities:

$$
Y_{\text {source }}=\tanh ^{-1}\left(V_{\text {source }}\right) \text {. }
$$

For the 80 and $100 \mathrm{MeV} / \mathrm{u}$ reactions the inclusive emission velocities were determined from the minor axis $\left(v_{\perp}\right)$ of the elliptical fits. The emission velocity can also be calculated from the coincidence events as the velocity in the center-of-mass frame. To calculate this velocity, a general Lorentz transformation was applied, in which the 
momentum vector of each fragment was transformed into the center-of-mass frame (not necessarily along the beam direction).

The components of the center-of-mass momentum vector are:

$$
\begin{aligned}
& P_{\| C M}=\sum_{i=1}^{n} P_{i} \cos \theta_{i} \\
& P_{X C M}=\sum_{i=1}^{n} P_{i} \sin \theta_{i} \cos \phi_{i} \\
& P_{y C M}=\sum_{i=1}^{n} P_{i} \sin \theta_{i} \sin \phi_{i} .
\end{aligned}
$$

Then

$$
\begin{aligned}
& P_{C M}=\sqrt{P_{\| C M}{ }^{2}+P_{x C M}{ }^{2}+P_{y C M}{ }^{2}} \\
& E_{C M}=\sum_{i=1}^{n} E_{k i n i}+M_{i}
\end{aligned}
$$

and the corresponding components of the center-of-mass velocity are:

$$
\begin{aligned}
& v_{\| C M}=\frac{P_{\| C M}}{E_{C M}} \\
& v_{x C M}=\frac{P_{X C M}}{E_{C M}} \\
& v_{y C M}=\frac{P_{Y C M}}{E_{C M}},
\end{aligned}
$$


and the magnitude of the center-of-mass velocity vector is:

$$
v_{C M}=\frac{P_{C M}}{E_{C M}}
$$

With

$$
\gamma=\frac{E_{\mathrm{CM}}}{\sum_{\mathrm{i}=1}^{\mathrm{n}} \mathrm{M}},
$$

we have the general Lorentz transformation for each fragment $\mathrm{i}$ :

$$
\begin{aligned}
& P_{\| H_{i}}=P_{\|_{i}}+\gamma_{\|_{C M}}\left[\frac{\gamma_{v_{C M}} P_{i} \cos \alpha}{\gamma+1}-\left(E_{k i n_{i}}+M_{i}\right)\right] \\
& \mathrm{P}_{x_{\mathrm{i}}}{ }=\mathrm{P}_{\mathrm{x}_{\mathrm{i}}}+\gamma_{x_{\mathrm{CM}}}\left[\frac{\gamma_{\mathrm{CM}} \mathrm{P}_{\mathrm{i}} \cos \alpha}{\gamma+1}-\left(\mathrm{E}_{\mathrm{kin} n_{\mathrm{i}}}+\mathrm{M}_{\mathrm{i}}\right)\right] \\
& P_{y_{i}}{ }^{\prime}=P_{y_{i}}+\gamma v_{Y_{C M}}\left[\frac{w_{C M} P_{i} \cos \alpha}{\gamma+1}-\left(E_{k i n_{i}}+M_{i}\right)\right] \\
& P_{i}=\sqrt{P_{\| i}{ }^{\prime 2}+P_{x i}{ }^{2}+P_{y i}{ }^{\prime 2}} \\
& E_{i}^{\prime}=\gamma\left(E_{k i n i}+M_{i}\right)-\gamma P_{i} v_{i} \cos \alpha \\
& v_{i}^{\prime}=\frac{P_{i}^{\prime}}{E_{i}^{\prime}} .
\end{aligned}
$$

Here $\alpha$ is the angle between the center-of-mass momentum vector and the fragment momentum vector. 
To calculate the mass transfer ( $A_{\text {Trane }}$ ) in the incomplete fusion process, we can assume that all of the beam momentum $\left(\mathrm{P}_{\text {beam }}\right)$ is given to the incomplete fusion product. Then

$$
\begin{aligned}
& P_{\text {beam }}=P_{\mathrm{IF}}=\sqrt{E_{I F}{ }^{2}-M_{\mathrm{IF}^{2}}} \\
& P_{\text {beam }}{ }^{2}=u^{2}\left(\gamma^{2}-1\right)\left(A_{\mathrm{P}}+A_{\text {Truss }}\right)^{2},
\end{aligned}
$$

where $u$ is the energy equivalent of an atomic mass unit $\left(931.5 \mathrm{MeV} / \mathrm{c}^{2}\right), A_{P}$ is atomic number of the projectile, and we have used $\mathrm{E}=\gamma \mathrm{M}$. Solving the above quadratic equation for ATrans gives:

$$
A_{\text {Trnos }}=\frac{P_{\text {beam }}}{u \sqrt{p^{2}-1}} \cdot A_{P}
$$

But

$$
\gamma=\frac{1}{\sqrt{1-v^{2} / c^{2}}} \quad \text { and } \quad \frac{v}{c}=\tanh (Y)
$$

so substitution into (A37) gives:

$$
A_{T r a s}=\frac{P_{\text {beam }}}{u \sinh (Y)}-A P
$$

The excitation energy of the incomplete fusion product can be calculated from:

$$
E^{*}=E_{\text {beam }}-E_{I F}+Q=E_{\text {beam }}-u \cosh (Y)\left(A_{P}+A_{\text {Trans }}\right)+Q
$$


where we have made the substitution $\gamma=\cosh (Y)$, and $Q$ is the ground state $Q$-value of the incomplete fusion process. 


\section{APPENDIX II: AN INCOMPLETE FUSION MODEL}

We can attempt to gain some insight into the incomplete fusion process by considering a very simple model that incorporates kinematics into a standard geometrical picture of a nucleus - nucleus interaction [Mo 86].

Let us assume that a nucleus of mass $A$ and velocity $V$ collides with a target nucleus of mass $B$ at an impact parameter $b$. There will be a portion of each nucleus that is occluded by the other. Let the occluded portions of $A$ and $B$ have masses of $\alpha$ and $\beta$, respectively. In the geometrical spirit of the model we can imagine three scenarios: 1) The piece $\beta$ is sheared off and attaches itself to $A, 2)$ The piece $\alpha$ is sheared off and attaches itself to B, or 3) Both pieces, $\alpha$ and $\beta$, are separated from A and B. Scenarios 1) and 2) correspond to the incomplete fusion process, while scenario 3) is the fireball model proposed for reactions at bombarding energies $>100 \mathrm{MeV} / \mathrm{u}$ [We 76, Go 77]. The processes in scenarios 1) and 2) are completely kinematical and can be theoretically studied without introducing dynamical assumptions, while scenario 3) requires dynamical information, such as restoring forces, to be included. It may be instructive to first investigate the simpler cases of scenarios 1) and 2) by assuming that either $\alpha$ or $\beta$, but not both, will be sheared off.

Let us assume that during the collision the portion $\beta$ attach itself to $A$. If the force generated between $A+\beta$ and $B-\beta$ is cs where $s$ is the distance between $A+\beta$ and $B-\beta$, then the velocities are:

$$
v_{A+\beta}=v_{\beta} \cdot \frac{1}{(A+\beta)} \int \operatorname{csd}, ; \quad v_{B-\beta}=\frac{1}{(B-\beta)} \int \operatorname{cs} d t,
$$

where $v_{\beta}$ is the intial velocity given to $\beta$ by $A, v_{\beta}=\frac{A}{A+\beta} v$. With $v_{S}=v_{A+\beta}-v_{B-\beta}$ we have the differential equation of motion: 


$$
\frac{d v_{s}}{d t}=-\frac{1}{m \beta^{*}} c s,
$$

where $m_{\beta} *=\frac{(A+\beta)(B-\beta)}{A+B}$. Integrating this equation yields:

$$
v_{A+\beta}-v_{B-\beta}=v_{\beta} \sqrt{1-\frac{c s^{2}}{m_{\beta}^{*} v_{\beta}^{2}}}
$$

Introducing momentum conservation $(A+\beta) v_{\beta}=(A+\beta) v_{A+\beta}+(B-\beta) v_{B-\beta}$ gives for the two velocities:

$$
\begin{aligned}
& v_{A+\beta}=v_{\beta}\left[1-\frac{B-\beta}{A+B}\left(1-\sqrt{1-\frac{2 \Delta \beta}{m \beta^{*} v_{\beta}^{2}}}\right)\right] \\
& v_{B-\beta}=v_{\beta} \frac{A+\beta}{A+B}\left(1-\sqrt{1-\frac{2 \Delta \beta}{m \beta^{*} v_{\beta}^{2}}}\right),
\end{aligned}
$$

where $\Delta \beta$ is the separation energy of $\beta$ when fracture of $B$ occurs at the maximum elongation $s_{\max }, \Delta_{\beta}=\frac{1}{2} c s_{\max }{ }^{2}$.

A well-studied parameter in intermediate energy reactions is the momentum transfer [Vi 82]. From equations (A44) the momentum of the pieces can be easily determined:

$$
\begin{aligned}
& \frac{P_{A+\beta}}{P_{A}}=1-\frac{B-\beta}{A+B}\left(1-\sqrt{1-\frac{2 \Delta_{\beta}}{m \beta^{*} v_{\beta}^{2}}}\right) \\
& \frac{P_{B-\beta}}{P_{A}}=\frac{B-\beta}{A+B}\left(1-\sqrt{1 \cdot \frac{2 \Delta_{\beta}}{m \beta^{*} v_{\beta}^{2}}}\right),
\end{aligned}
$$

where $P_{A}$ is the momentum of the beam. The momentum of the pieces in scenario (2) can be calculated in the same fashion: 


$$
\begin{aligned}
& \frac{P_{B+\alpha}}{P_{A}}=\frac{\alpha}{A}+\frac{B(A-\alpha)}{A(A+B)}\left(1-\sqrt{1-\frac{2 \Delta_{\alpha}}{m_{\alpha}^{*} v_{\alpha}^{2}}}\right) \\
& \frac{P_{A-\alpha}}{P_{A}}=\frac{A-\alpha}{A}-\frac{B(A-\alpha)}{A(A+B)}\left(1-\sqrt{1-\frac{2 \Delta_{\alpha}}{m_{\alpha}^{*} v_{\alpha}^{2}}}\right),
\end{aligned}
$$

here $m_{\alpha}{ }^{*}=\frac{(A-\alpha)(B+\alpha)}{A+B}$ and $v_{\alpha}=\frac{B}{B+\alpha} v$. It is interesting to note that full momentum transfer (complete fusion) occurs when the square roots in (A45) and (A46) vanish:

$$
\frac{2 \Delta_{\beta}}{m \beta^{*} v_{0}}=1 \quad \text { and } \quad \frac{2 \Delta_{\alpha}}{m_{\alpha^{*} y_{0}}}=1
$$

This occurs when the bombarding energy per nucleon of $A$ is:

$$
\frac{E}{A}=\frac{\Delta \beta(A+\beta)(A+B)}{(B-\beta) A^{2}} \text { and } \quad \frac{E}{A}=\frac{\Delta \alpha(B+\alpha)(A+B)}{(A-\alpha) B^{2}}
$$

Notice also from (A45) and (A46) that at very large bombarding energies the momentum transfers asymptotically tend to the values one would have without binding, where $\Delta_{\alpha}=0$ and $\Delta_{\beta}=0$.

The separation energies, $\Delta_{\alpha}$ and $\Delta_{\beta}$, and masses, $\alpha$ and $\beta$, can be calculated from the geometrical aspects of the problem. We take the separation energy as the energy necessary to create new nuclear surface. This can be calculated from the area of the surface created when the projectile nucleus $A$ drills a hole through the target nucleus $\mathrm{B}$, or vice versa. If we assume $A$ and $B$ to be spherical, then the area of the cylindrical surface that is created can be calculated analytically. If $R$ is the radius of the sphere (drilled-out nucleus), $r$ the radius of the cylinder (drilling nucleus), and $b$ the impact parameter, then 
the new surface area $S$ is:

$$
S=8 r \sqrt{R^{2}-r^{2}-b^{2}+2 b r} E(\pi / 2, k) \quad k=\sqrt{\frac{4 b r}{R^{2}-r^{2}-b^{2}+2 b r}}
$$

for $R^{2}-r^{2}-b^{2}>2 b r>0$, and

$$
S=4 r \sqrt{\frac{1}{b r}}\left(\left(R^{2}-r^{2}-b^{2}-2 b r\right) F(\pi / 2,1 / k)+4 b r E(\pi / 2,1 / k)\right)
$$

for $\left|R^{2}-r^{2}-b^{2}\right|<2 b r$, where $E$ and $F$ are the elliptic integrals of the 1 st and 2 nd kind. The separation energy, $\Delta$, is then $\Delta=2 s_{0} S$ because the total new surface created is twice the cylindrical area. The nuclear surface energy constant $s_{0}$, is $s_{0} \equiv 0.95 \mathrm{MeV} / \mathrm{fm}^{2}$.

To determine $\alpha$ or $\beta$, the mass of the detached piece, requires the calculation of the volume of the spherical target swept out by the projectile. Following Swiatecki [Sw 76, Go 77], the volumes can be approximately calculated analytically as:

$$
\beta=B F(v, \gamma) \quad \text { and } \alpha=A F(v, \gamma) \text {, }
$$

where $F$ is a function (given below) of the dimensionless parameter $v$ specifying the relative sizes of the projectile and target ( $R$ and $r$ ), and of the dimensionless variable $\gamma$ specifying the impact parameter (b):

$$
v=\frac{R}{R+r} \quad ; \quad \gamma=\frac{b}{R+r} .
$$

The approximate formulae for $F$ are:

$$
F_{I} \pi\left[1-\left(1-\mu^{2}\right)^{3 / 2}\right] \sqrt{1-(\gamma / v)^{2}} \quad \text { for } v>\frac{(1+\gamma)}{2}
$$




$$
\begin{array}{ll}
F_{I I}=\frac{3}{4} \sqrt{1-v}\left(\frac{1-\gamma}{v}\right)^{2}-\frac{1}{8}\left\{\frac{3 \sqrt{1-v}}{\mu}-\frac{\left[1-\left(1-\mu^{2}\right)^{3 / 2}\right] \sqrt{1-(1-\mu)^{2}}}{\mu^{3}}\right\}\left(\frac{1-\gamma}{v}\right)^{3} & \text { for } \frac{(1+\gamma)}{2}>v>\frac{1}{2} \\
F_{I I I}=\frac{3}{4} \sqrt{1-v}\left(\frac{1-\gamma}{v}\right)^{2}-\frac{1}{8}\{3 \sqrt{1-v}-1\}\left(\frac{1-\gamma}{v}\right)^{3} & \text { for } \frac{1}{2}>v>\frac{(1-\gamma)}{2} \\
F_{I V}=1 & \text { for } \frac{(1-\gamma)}{2}>v .
\end{array}
$$

Here the abbreviation $\mu=\frac{1}{v}-1=\frac{r}{R}$ has been used.

The reaction ${ }^{93} \mathrm{Nb}+{ }^{9} \mathrm{Be}$ has been studied both experimentally and theoretically at several energies between 11 and $30 \mathrm{MeV} / \mathrm{u}$ [Ch 88a, Ch 88b]. Figure A.1 shows the impact parameters in which incomplete fusion processes become energetically possible in this reaction. At impact parameters less than $-3 \mathrm{fm}$ the ${ }^{9} \mathrm{Be}$ nucleus is completely occluded by the ${ }^{93} \mathrm{Nb}$ and no breakup of the ${ }^{9} \mathrm{Be}$ is possible. For each impact parameter, there is a lower energy limit given by (A47) above at which neither $\alpha$ or $\beta$ can be detached. This energy is the upper limit for complete fusion at the given impact parameter. This threshold moves to lower energies with increasing impact parameter for geometric reasons. At the largest impact parameters, the shearing process can be associated with quasi-elastic and direct reactions. At the upper energy range, where both ${ }^{93} \mathrm{Nb}$ and ${ }^{9} \mathrm{Be}$ breakup are possible, we expect incomplete fusion to end and give way to the fireball process.

Figure A.2 shows the momentum transfered to the target-like $\left({ }^{93} \mathrm{Nb}\right)$ nucleus in reactions in which a portion of the ${ }^{9} \mathrm{Be}$ projectile is absorbed as a function of bombarding energy, and purametrically, as a function of impact parameter. Figure A.3 shows the complementary momentum remaining with the ${ }^{9} \mathrm{Be}$ residue in the same reaction. In Figure 
A.4 the fractional momentum of the ${ }^{93} \mathrm{Nb}$-like fragment has been integrated over impact parameter with the proper geometric weighting (b). The points with error bars are the experimentally determined momentum transfers in the ${ }^{93} \mathrm{Nb}+{ }^{9} \mathrm{Be}$ system [Ch $88 \mathrm{~b}$ ]. The dashed line is the momentum transfer systematics [Vi 82, Ga 82, Ch 83b, Mo 84, St 85]. The model predictions are somewhat lower than the systematic data at bombarding energies $540 \mathrm{MeV} / \mathrm{u}$. However, the experimental points are the average momentum transfers associated with fission or complex fragment emission. The potential barriers for these processes can make them rare events, especially at small bombarding energy, and they will predominandy occur in the events with the largest energy depositions, and hence the largest momentum transfers. Thus, it is not too surprising that below $40 \mathrm{MeV} / \mathrm{u}$ the experimental momentum transfer associated with fission and complex fragment emission would be larger than that predicted by geometrically weighting over the allowed range of impact parameters.

Notice the asymptotic behavior of the momentum transfer above $-30 \mathrm{MeV} / \mathrm{u}$ in Figures A.2 - A.4. This predicted asymptotic behavior is consistent with the experimental results in both the ${ }^{93} \mathrm{Nb}+{ }^{9} \mathrm{Be}$ reactions at 25 and $30 \mathrm{MeV} / \mathrm{u}$ [Ch $88 \mathrm{a}$ ], and in the ${ }^{139} \mathrm{La}+$ $12 \mathrm{C}$ reactions discussed in this thesis (see table III.1)

Using this model, it is also possible to calculate the spins and excitation energies of the incomplete fusion products in order to simulate their decay with statistical models. The spin transferred to the incomplete fusion product has been calculated by numerically integrating over the volume of the transferred piece to find its center of mass relative to the center $f$ mass of the receiving nucleus. The excitation energy of the incomplete fusion product can be approximated by $E^{*}=E A \frac{\beta A}{\beta+A}+s_{0} S+Q$, where $E A$ is the bombarding energy per nucleon, $\beta$ is the mass of the transferred piece, $A$ is the mass of the receiving nucleus, $Q$ is the ground state mass difference of the entrance and exit channel fragments, and so and $S$ are defined as above.

The model has been used to calculate the spins and excitation energies of complete 
and incomplete fusion products in the $30.3 \mathrm{MeV} / \mathrm{u}{ }^{93} \mathrm{Nb}+{ }^{9} \mathrm{Be}$ reaction as shown in Figure A.5 [Ch 88a]. For entrance channel angular momenta $<37 \mathrm{~K}$, the model predicts complete fusion, therefore the excitation energy is constant. At larger $\ell$-waves, the incomplete fusion process sets in and both the transferred spin and excitation energy decrease with increasing \&-wave. In Figure III.24 the calculated spins and excitation energies have been used as input for statistical decay calculations. The cross sections are presented as a function of the entrance channel $l$-wave. The points at which the cross sections drop dramatically are where the model predicts the incomplete fusion process to begin. Most of the $\mathrm{Z}=20$ yield is predicted to arise from complete fusion in the $25.4 \mathrm{MeV} / \mathrm{u}{ }^{93} \mathrm{Nb}+{ }^{9} \mathrm{Be}$ reaction, while instead most of the yield is predicted to arise from incomplete fusion in the case of $30.3 \mathrm{MeV} / \mathrm{u}{ }^{93} \mathrm{Nb}+{ }^{27} \mathrm{Al}$ [Ch $\left.88 \mathrm{a}\right]$.

The excitation energy of the products in these reactions should be calculated by taking into account the difference in surface areas of the products just after the mass transfer has occurred and at equilibrium ( spherical configuration). For the incomplete fusion product, most of the excitation energy is due to mass transfer rather than to surface relaxation, thus calculating the difference in surface areas explicitly will have a negligible effect on the total excitation energy. However, for the spectator fragment which loses a portion of its mass, the difference in surface areas is equal to the total excitation energy, and should be calculated as accurately as possible.

This problem has also been investigated by Swiatecki [Sw 76, Go 77]. He calculated the excess surface area above that of the spherical equilibrium configuration, and the corresponding excitation energy as approximately:

$$
\Delta A=4 \pi R^{2}\left(1+P \cdot(1-F)^{2 / 3}\right) \quad \text { and } \quad E^{*}=\Delta A s_{0} .
$$

where $R$ is the (initial) radius of the spectator fragment, $F$ are given by (A53) above and $P$ are given by the following formulae: 


$$
\begin{aligned}
& P_{I}=\left(\frac{1}{v} \sqrt{1-\mu^{2}}-1\right) \sqrt{1-(\gamma / v)^{2}} \\
& P_{\text {II }}=\frac{1}{8} \sqrt{\mu v\left(\frac{1}{\mu}-2\right)\left(\frac{1-\gamma}{v}\right)^{2}-} \\
& \qquad \frac{1}{8}\left\{\frac{1}{2} \sqrt{\left.\frac{v}{\mu}\left(\frac{1}{\mu}-2\right)-\frac{\left(\frac{1}{v} \sqrt{1-\mu^{2}}-1\right) \sqrt{(2-\mu) \mu}}{\mu^{3}}\right\}\left(\frac{1-\gamma}{v}\right)^{3}}\right. \\
& P_{\text {III }}=\frac{1}{8} \sqrt{\mu v}\left(\frac{1}{\mu}-2\right)\left(\frac{1-\gamma}{v}\right)^{2}-\frac{1}{8}\left\{\frac{1}{2} \sqrt{\mu v}\left(\frac{1}{\mu}-2\right)+1\right\}\left(\frac{1-\gamma}{v}\right)^{3} \\
& \text { PIV }=-1 .
\end{aligned}
$$

$P_{1}$ - $F_{I V}$ are used in the same geometrical regions as specified above for $F_{I}-F_{I V}$.

These formulae also give the excitation energies of the spectator fragments in the fireball scenario [(3) above]. According to the model, the excitation energy of the spectator fragments should be insensitive to the bombarding energy. Evidence for this excitation energy saturation could be found by observing crcss sections that are constant over a wide range of bombarding energy. However, one should remember that important considerations have been neglected. While the effect of the finite range potential should decrease the calculated excitation energies, nucleon scattering from the fireball into the spectator fragments might increase the excitation energies substantially. 

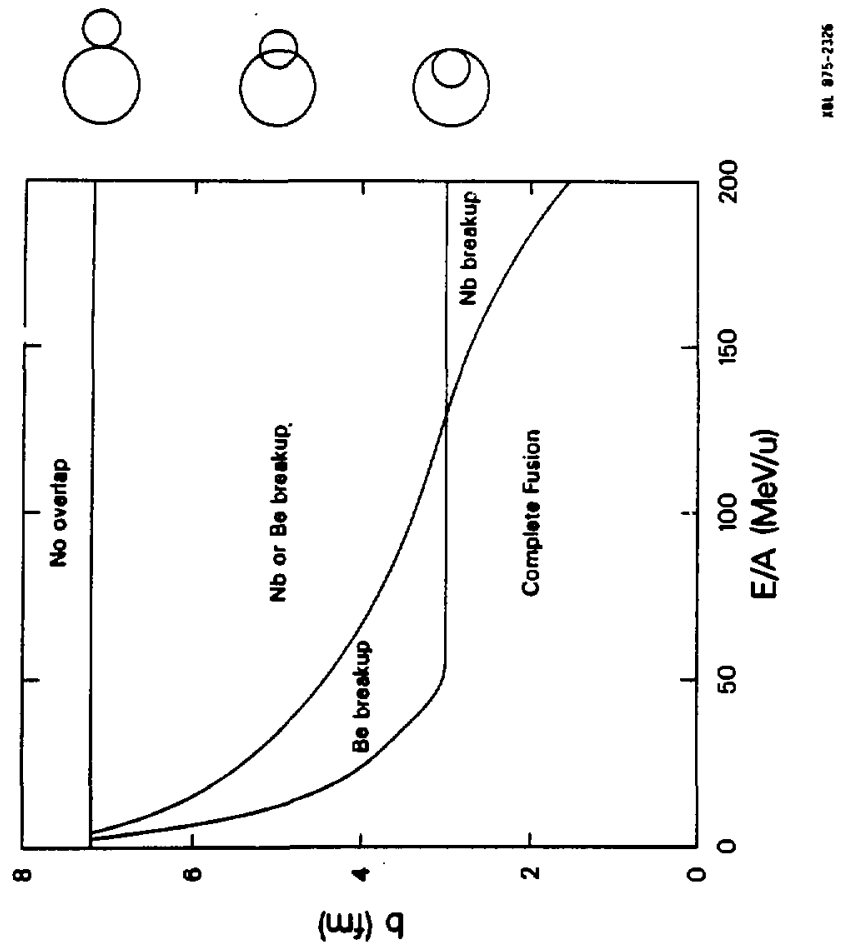

Figure A.1 The predicted impact parameters, as a function of bombarding energy, at which it is possible to shear off a portion of the target or projectile in the reaction ${ }^{93} \mathrm{Nb}+$ ${ }^{9} \mathrm{Be}$. The schematic drawings at the right show the reaction configurations at impact parameters corresponding to touching, partial overlap, and complete overlap. 


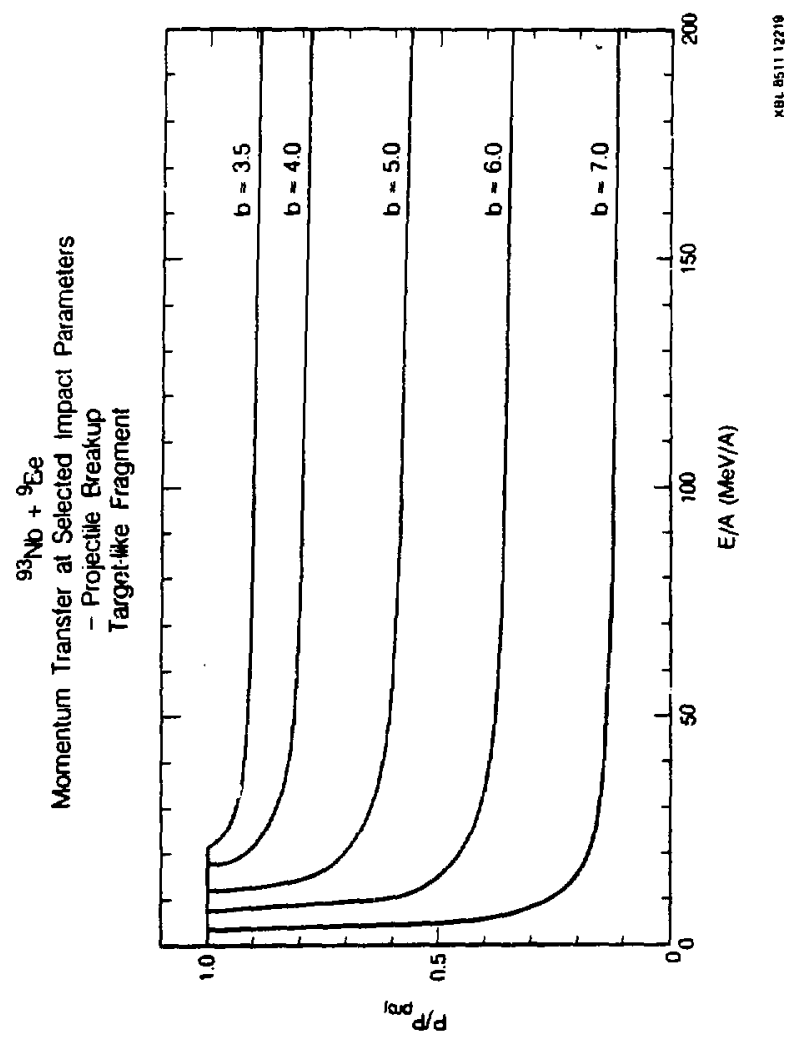

Figure A.2 The momentum transferred to the target-like $\left({ }^{93} \mathrm{Nb}\right)$ fragment in the reaction of ${ }^{9 \mathrm{Be}}+{ }^{93} \mathrm{Nb}$ as a function of bon:bazding energy, and parametrically, of impact parameter. A portion of the ${ }^{9} \mathrm{Be}$ projectile is assumed to be absorbed by the ${ }^{93} \mathrm{Nb}$ target. 


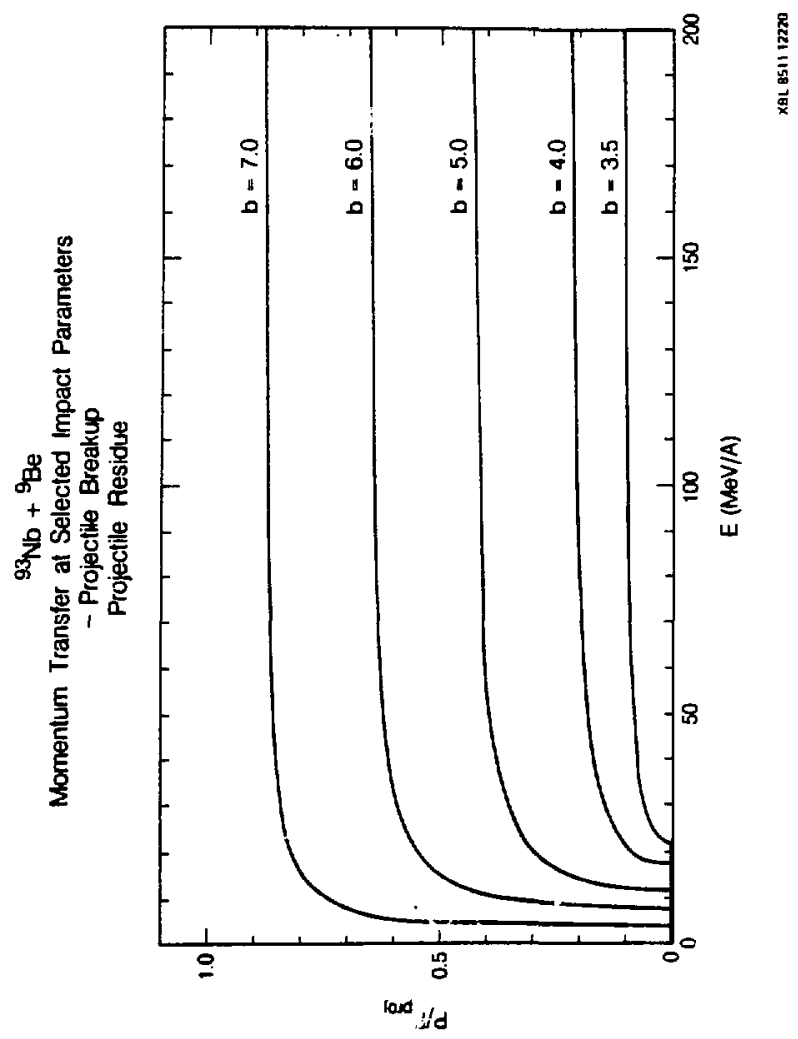

Figure A.3 The momentum remaining in the projectile-like $\left({ }^{9} \mathrm{Be}\right)$ fragment in the reaction of ${ }^{9} \mathrm{Be}+{ }^{93} \mathrm{Nb}$ as a function of bombarding energy, and parametrically, of impact parameter. This Figure is the complement of Figure A.2. 


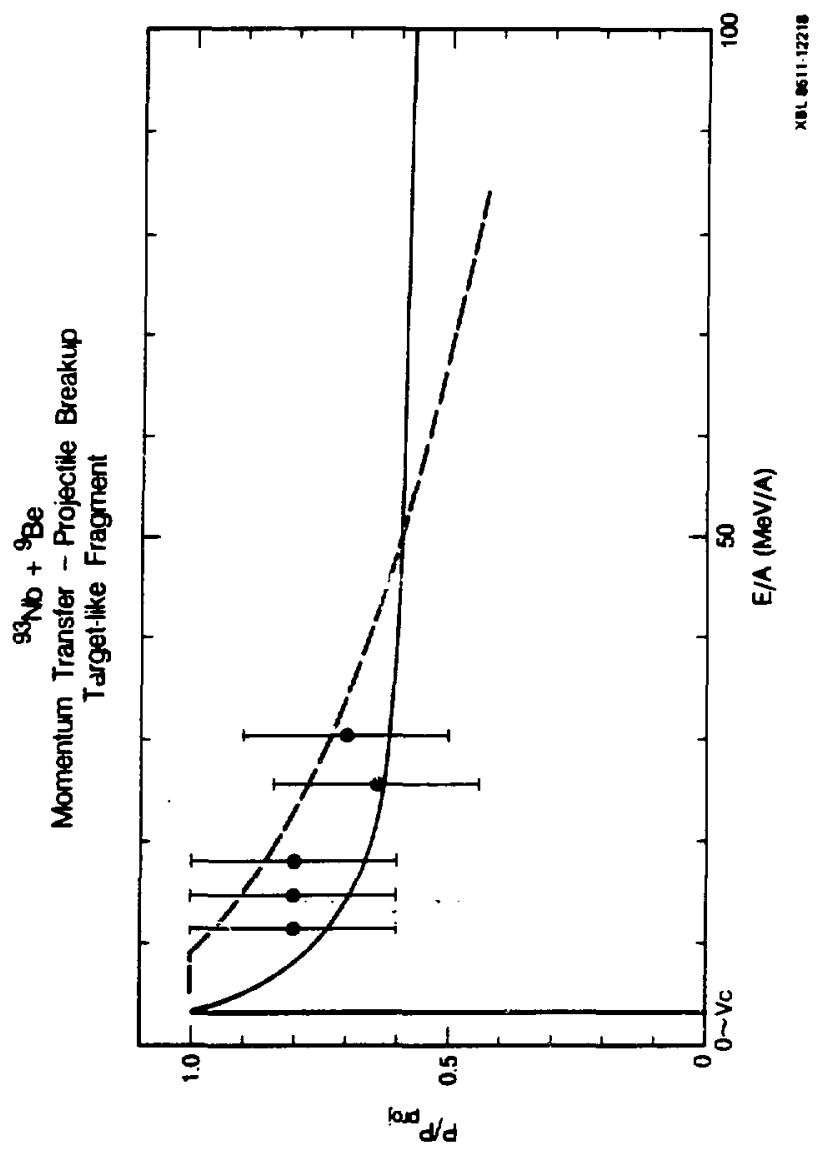

Figure A.4 The solid lines represent the momentum transferred to the target-like $\left({ }^{93} \mathrm{Nb}\right)$ fragment in the reaction of ${ }^{9} \mathrm{Be}+{ }^{93} \mathrm{Nb}$ as a function of bombarding energy. The momentum transfer has been integrated over impact parameter with the proper geometric weighting (b). The solid points and error bars are the experimental momentum transfers associated with complex fragment emission in the ${ }^{93} \mathrm{Nb}+{ }^{9} \mathrm{Be}$ reaction at the given bombarding energies [ $\mathrm{Ch} 88 \mathrm{a}, \mathrm{Ch} 88 \mathrm{~b}$ ]. The dashed line is the experimental momentum transfer systematics [Vi 82, Ga 82, Ch 83b, Mo 84, St 85]. 
$30.3 \mathrm{MeV} / \mathrm{u} \mathrm{Nb}+\mathrm{Be}$

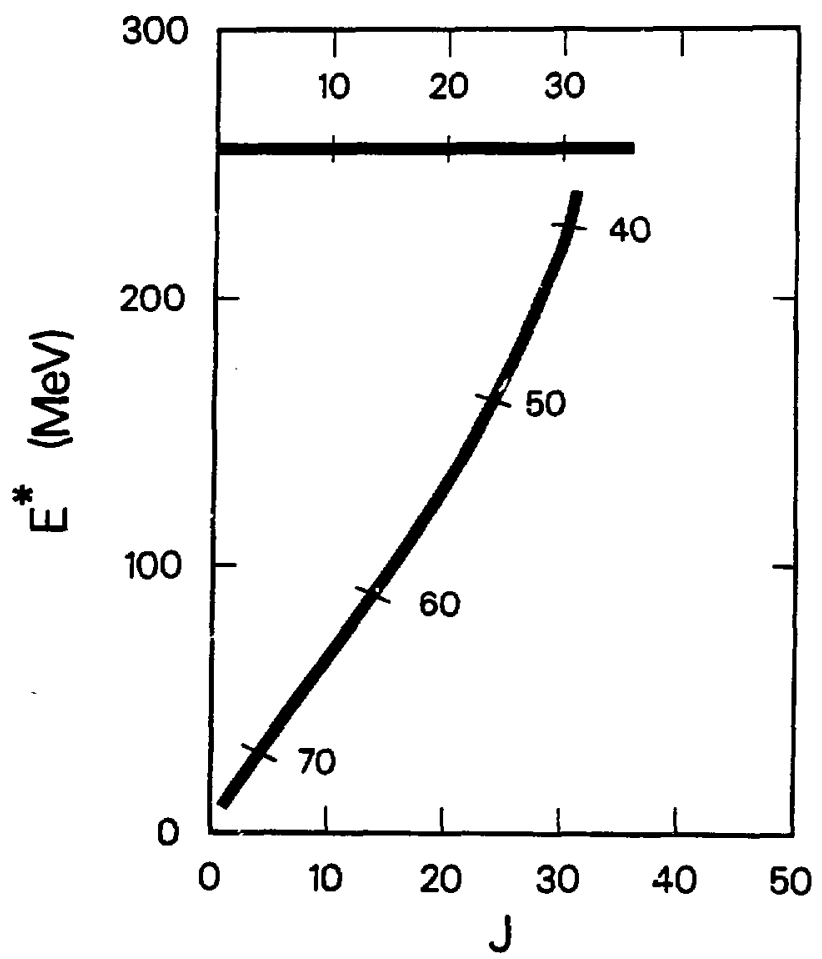

XBL $875-2336$

Figure A.5 The predicted excitation energy of the compound system plotted as a function of its predicted spin in the $30.3 \mathrm{MeV} / \mathrm{u}{ }^{93} \mathrm{Nb}+{ }^{9} \mathrm{Be}$ reaction. The entrance channel l-waves corresponding to the $E^{*}$ and $J$ values are shown. At $l$-waves $>37$ complete fusion process gives w'yy to incomplete fusion of a portion of the ${ }^{9} \mathrm{Be}$ target with the ${ }^{93} \mathrm{Nb}$ projectile [Ch 88a]. 


\section{FOOTNOTES}

1The angular distributions can be expressed in terms of $d \sigma / d \Omega$ or $d \sigma / d \theta$. In the $d \sigma / d \theta$ representation, "isotropic" or "flat" should be read as "isotropic in the reaction plane". Isotropic distributions in the reaction plane ( $\mathrm{d} \sigma / \mathrm{d} \theta=$ constant) are equivalent to $\mathrm{d} \sigma / \mathrm{d} \Omega \propto$ $1 / \sin \theta$, which is the equilibrium distribution of fragments emitted from systems with high angular momentum. Unless otherwise noted, we will always refer to angular distributions in terms of $d \sigma / d \theta$.

2By "Coulomb-like" velocities we mean that the relative energy between the fragment $\left(Z_{1}, A_{1}\right)$ and its decay partner $\left(Z_{2}, A_{2}\right)$ is approximately given by the parametrization:

$$
E_{\text {Coul }}=1.44 \frac{Z_{1} Z_{2}}{I_{0}\left(A_{1}^{1 / 3}+A_{2}^{1 / 3}\right)+2}
$$

with $r_{0} \equiv 1.2 \mathrm{fm}$. This parametrization has successfully reproduced the relative energies between the partners in completely relaxed deep-inelastic reactions, and, fcr symmetric decay, gives relative energies very close to the Viola fission fragment systematics [ $\mathrm{Vi} 85$ ].

${ }^{3}$ The Lorentz-invariant cross section, $\frac{d^{2} \sigma}{f d v d \Omega}$, can be calculated from:

$$
f d v d \Omega=f d v^{\prime} d \Omega^{\prime} \frac{f \partial(v, \Omega)}{f^{\prime} \partial\left(v^{\prime}, \Omega^{\prime}\right)}
$$

when the Jacobian of the transformation $\frac{f(v, \Omega)}{f^{\prime} \partial\left(v^{\prime}, \Omega^{\prime}\right)}=1$. Here $f$ and $f^{\prime}$ are the factors to be determined. They can be calculated most easily from: 


$$
\begin{aligned}
\frac{f^{\prime}}{f}=\frac{\partial(v, \Omega)}{\partial\left(v^{\prime}, \Omega^{\prime}\right)} & =\frac{\partial(v, \Omega)}{\partial(p, \Omega)} \frac{\partial(p, \Omega)}{\partial\left(p^{\prime}, \Omega^{\prime}\right)} \frac{\partial\left(p^{\prime}, \Omega^{\prime}\right)}{\partial\left(v^{\prime}, \Omega^{\prime}\right)} \\
& =\frac{d v}{d p} \frac{\partial(p, \Omega)}{\partial\left(p^{\prime}, \Omega^{\prime}\right)} \frac{d p^{\prime}}{d v^{\prime \prime}}
\end{aligned}
$$

To calculate the Jacobian $\frac{\partial(\mathrm{p}, \Omega)}{\partial\left(\mathrm{p}^{\prime}, \Omega^{\prime}\right)}$ we remember

$$
d p_{x} d p_{y} d p_{z}=p^{2} \sin \theta d p d \theta d \phi
$$

The solid angle, $d \Omega$, is defined as, $d \Omega=\sin \theta d p d \theta$, so,

$$
d p_{x} d p_{y} d p_{z}=p^{2} d p d \Omega
$$

Similarly,

$$
d p_{x}^{\prime} d p^{\prime} d p_{z}^{\prime}=p^{2 \prime} d p^{\prime} d \Omega^{\prime}
$$

Now to determine the Jacobian $\frac{\partial\left(p_{x}, p_{y}, p_{z}\right)}{\partial\left(p_{x}{ }^{\prime}, p_{y}{ }^{\prime}, p_{z}{ }^{\prime}\right)}$, we choose a coordinate system such that the relative motion is along the $z$ axis, so $d p_{x}=d p_{x}^{\prime}$ and $d p_{y}=d p_{y}^{\prime}$. The Jacobian we are

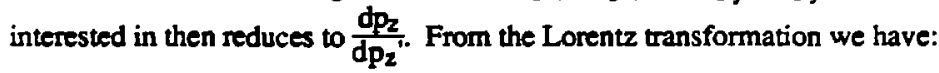

$$
\begin{aligned}
& p_{z}=\gamma\left(p_{z^{\prime}}+\beta E^{\prime}\right) \\
& E=\gamma\left(E^{\prime}+\beta p_{z}^{\prime}\right) \\
& \frac{d p_{z}}{d p_{z}^{\prime}}=\gamma\left(1+\beta \frac{d E^{\prime}}{d p_{z}^{\prime}}\right) .
\end{aligned}
$$


With $\frac{d E^{\prime}}{d p_{z}^{\prime}}=\frac{p_{z}^{\prime}}{E^{\prime}}$, equation (F9) becomes:

$$
\frac{d p_{z}}{d p_{z}^{\prime}}=\gamma\left(1+\beta \frac{p_{z}^{\prime}}{E^{\prime}}\right)=\frac{E}{E^{\prime}}=\frac{\gamma}{\gamma}
$$

Hence, from (F5), (F6), and (F10) we have:

$$
\frac{\partial(p, \Omega)}{\partial\left(p^{\prime}, \Omega^{\prime}\right)}=\frac{p^{\prime 2}}{p^{2}} \frac{\partial\left(p_{x}, p_{y}, p_{z}\right)}{\partial\left(p_{x}^{\prime}, p_{y}^{\prime}, p_{z}^{\prime}\right)}=\frac{p^{\prime 2}}{p^{2}} \frac{d p_{z}}{d p_{z}^{\prime}}=\frac{p^{\prime 2} \gamma}{p^{2} \gamma}
$$

The other factors in (F3) can be found from:

$$
\begin{aligned}
& p=\gamma m v=\frac{m v}{\sqrt{1-\frac{v^{2}}{c^{2}}}} \\
& \frac{d p}{d v}=m \gamma\left(\frac{v^{2} \gamma^{2}}{c^{2}}+1\right)=m \gamma^{3},
\end{aligned}
$$

and of course,

$$
\frac{d p^{\prime}}{d v^{\prime}}=m \gamma^{3}
$$

Then from (F11), (F13), and (F14):

$$
\frac{f^{\prime}}{f}=\frac{\partial(v, \Omega)}{\partial\left(v^{\prime}, \Omega^{\prime}\right)}=\frac{\gamma^{2} p^{\prime 2}}{\gamma^{2} p^{2}}=\frac{\gamma^{4} v^{\prime 2}}{\gamma^{4} v^{2}} .
$$

Hence, the Lorentz-invariant cross section, $\frac{d^{2} \sigma}{f d v d \Omega}$, is $\frac{d^{2} \sigma}{\gamma^{4} v^{2} d v d \Omega}$. Note that at small 
velocity where $\gamma \cong 1$ this cross section approaches the Galilean-invariant cross section $\frac{d^{2} \sigma}{v^{2} d v d \Omega}$ (as it must). At $50 \mathrm{MeV} / \mathrm{u}$, the use of the Galilean-invariant, rather than the Lorentz-invariant, cross section introduces errors in the cross section at the $20 \%$ level.

${ }^{4}$ In this case we want to calculate the Lorentz-invariant cross section $\frac{d^{2} \sigma}{f d v_{\|} d v_{\perp}}$. As abo' $e$, the cross section is invariant when the Jacobian of the transformation equals one:

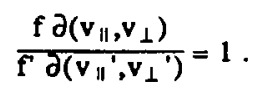

Hence,

$$
\begin{aligned}
& \frac{f^{\prime}}{f}=\frac{\partial\left(v_{\|}, v_{\perp}\right)}{\partial\left(v_{\|}{ }^{\prime}, v_{\perp}{ }^{\prime}\right)}=\frac{\partial\left(v_{\|}, v_{\perp}\right)}{\partial\left(v_{\|}, p_{\perp}\right)} \frac{\partial\left(v_{\|}, p_{\perp}\right)}{\partial\left(p_{\|}, p_{\perp}\right)} \frac{\partial\left(p_{\|}, p_{\perp}\right)}{\partial\left(p_{\|}{ }^{\prime}, p_{\perp}{ }^{\prime}\right)} \frac{\partial\left(p_{\|}, p_{\perp}{ }^{\prime}\right)}{\partial\left(v_{\|}{ }^{\prime}, p_{\perp}{ }^{\prime}\right)} \frac{\partial\left(v_{\|}{ }^{\prime}, p_{\perp}{ }^{\prime}\right)}{\partial\left(v_{\|}, v_{\perp}{ }^{\prime}\right)} \\
& =\frac{d v_{\perp}}{d p_{\perp}} \frac{d v_{\|}}{d p_{\|}} \frac{\partial\left(\dot{p}_{\|}, p_{\perp}\right)}{\partial\left(p_{\|}^{\prime}, p_{\perp}{ }^{\prime}\right)} \frac{d p_{\|}{ }^{\prime}}{d v_{\|}^{\prime}} \frac{d p_{\perp}{ }^{\prime}}{d v_{\perp}} .
\end{aligned}
$$

To determine $\frac{\partial\left(p_{\| t}, p_{\perp}\right)}{\partial\left(p_{\|}, p_{\perp}\right)}$ we write

$$
d p_{x} d p_{y} d p_{z}=p_{\perp} d p_{\|} d p_{\perp} d \phi \text {, }
$$

and

$$
d p_{x}^{\prime} d p_{y}^{\prime} d p_{z}^{\prime}=p_{\perp}^{\prime} d p_{\|}^{\prime} d p_{\perp}^{\prime} d \phi^{\prime} .
$$

Using (F7 - F10) and remembering that $d \phi=d \phi^{\prime}$, we can immediately write 


$$
\frac{\partial\left(p_{\|}, p_{\perp}\right)}{\partial\left(p_{\|}^{\prime}, p_{\perp}\right)}=\frac{p_{\perp}^{\prime}}{p_{\perp}} \frac{\partial\left(p_{x}, p_{y}, p_{z}\right)}{\partial\left(p_{x}^{\prime}, p_{y}^{\prime}, p_{z}^{\prime}\right)}=\frac{p_{\perp}^{\prime}}{p_{\perp}} \frac{d p_{z}}{d p_{z}^{\prime}}=\frac{p_{\perp}^{\prime} \gamma}{p_{\perp} \gamma}
$$

The other factors in (F17) can be calculated from:

$$
\frac{d p_{\|}}{d v_{\|}}=\frac{d p_{\|}}{d p} \frac{d p}{d v} \frac{d v}{d v_{\|}}=m \gamma^{3} \frac{p}{p_{\|}} \frac{v_{\|}}{v}=m \gamma^{3},
$$

and similarly:

$$
\frac{d p_{\perp}}{d v_{\perp}}=m \gamma^{3}
$$

Hence from (F20 - F22) we have:

$$
\frac{\partial\left(v_{11}, v_{\perp}\right)}{\partial\left(v_{11}, v_{\perp}\right)}=\frac{p_{\perp} \cdot \gamma^{5}}{p_{\perp} \gamma^{5}}=\frac{v_{\perp} \cdot \gamma^{6}}{v_{\perp} \gamma^{6}} \text {. }
$$

The Lorentz-invariant cross section is then $\frac{\partial^{2} \sigma}{\gamma_{v_{\perp}} \partial v_{\|} \partial v_{\perp}}$. However, to show the reaction plane isotropy, we have multiplied this cross section by $v_{\perp}$. The resulting cross section is invariant only to translations of $v_{\|}$for which $p_{\perp}=p_{\perp}$ : Hence, $\frac{\partial^{2} \sigma}{\gamma^{5} \partial v_{\|} \partial v_{\perp}}$ is the cross section that is Lorentz-invariant to translations of $\mathrm{v}_{\|}$. The use of the non-relativistic cross section $\frac{\partial^{2} \sigma}{v_{\perp} \partial \gamma_{\|} \partial v_{\perp}}$ at $50 \mathrm{MeV} / \mathrm{u}$ introduces errors of the order of $\gamma^{5}$ (20\% for the intermediate Z-values, $20 \leq \mathrm{Z} \leq 30$ ).

SThe errors introduced by using the Galilean transformation, rather than the Lorentz transformation, will depend upon the emission angle of the fragr.unt. The error at $90^{\circ}$ can 
be calculated quite easily. Here, vemiss $=v_{\perp}{ }^{\prime}$ (source frame). With $p_{\perp}=p_{\perp}{ }^{\prime}$, we have (relativistically) $v_{\perp}{ }^{\prime}=\frac{\gamma}{\gamma} v_{\perp}$, whereas non-relativistically we assume $v_{\perp}{ }^{\prime}=v_{\perp}$. Since $\gamma \equiv$ 1 , the error in using non-relativistic kinematics is $\gamma$. 


\section{REFERENCES}

Ab 86

Ai 84a

Ai $84 b$

Ai 85

Ai 86a

Ai $86 b$

Ai 87

Au 85

Au 86

Au 87

Ba. 74

Ba 76

Ba 77

Ba 8:

Ba $86 a$

Ba B6b

A.Y. Abul-Magd and D.H.E. Gross, Z. fur Phys. A325, 373 (1986).

J. Aichelin and J. Huefner, Phys. Lett. 136B, 15 (1984).

J. Aichelin, J. Huefner, and R. Tbarra, Phys. Rev. C 30, 107 (1984).

J. Aichelin and G. Bertsch, Phys. Rev. C 31, 1730 (1985).

J. Aichelin, J. Phys. C4, 63 (1986).

J. Aichelin and H. Stocker, Phys. Lett 176B, 14 (1986).

J. Aichelin, A. Rosenhauer, G. Peilert, H. Stocker, and W. Greiner, Phys. Rev. Lett. 58, 1926 (1987).

G. Auger, D. Jouan, E. Plagnol, F. Pougheon, F. Naulin, H. Doubre, and C. Gregoire, Z fur Phys. A321, 243 (1985).

G. Auger, E. Plagnol, D. Jouan. $r$ Guet, D. Heuer, M Maurel, H. Nifenecker, C. Ristori, F. Schı $\quad$ H. Doubre, and C. Gregoire, Phys. Lett. 169B, 161 (1986).

F. Auger, B. Berthier, A. Cunsolo, A. Foti, W. Mittig, J.M. Pascaud, E. Plagnol, J. Quebert, and J.P. Wieleczko, Phys. Rev. C 35, 190 (1987). R. Bass, Nucl. Phys. A231, 45 (1974).

R. Babinet, L.G. Moretto, J. Galin, R. Jared, J. Mos:ton, and S.G. Thompson, Nucl. Phys. A258, 172 (1976).

R. Bass, Phys. Rev. Lett. 39, 265 (1977).

W. Bauer, D.R. Dean, U. Mosel, and U. Post, Phys. Lett. 150B, 53 (1985).

H.W. Barz, J.P. Bondorf, R. Donangelo, I.N. Mishustin, and H. Schulz, Nucl. Phys. A448, 753 (1986).

W. Bauet, U. Post, D.R. Dean, and U. Mosel, Nucl. Phys. A452, 699 (1986). 
Be 36

Be 84

Bj 82

Bo 39

Bo 48

Bo 69

Bo 73

Bo 85a

Bo $85 b$

Bo 85c

Bo 87

Bo 88

Bu 55

Ca 83

Ca 88

Ce 88

H.A. Bethe, Phys. Rev. 50, 332 (1936).

G.F. Bertsch, H. Kruse, and S. Das Gupta, Phy/s. Rev. C 29, 673 (1984).

S. Bjomholm and W.J. Swiatecki, Nucl. Phys. A391, 471 (1982).

Niels Bohr and John Archibald Wheeler, Phys. Rev 56, 426 (1939).

N. Bohr, $\mathrm{K}_{\xi}$ : Danske Videnskab. Selskab, Mat.-fys. Medd., XVII, 8 (1948).

A. Bor and B. Mottelson, Nuclear Structure, Vol. I, (Benjamin, New York, 1969).

J.D. Bowman, W.J. Swiatecki , and C.F. Tsang, Lawrence Berkeley Laboratory publication LBL - 2908 (1973).

J.P. Bondorf, R. Donangelo, I.N. Mishustin, C.J. Pethick, H. Schulz, and K. Sneppen, Nucl. Phys. A443, 321 (1985).

J. Bondorf, R. Donangelo, I.N. Mishustin, and H. Schulz, Nucl. Phys. A444, 460 (1985).

B. Borderie and M.F. Rivet, Z. fur Phys. A321, 703 (1985).

D.R. Bowman, R.J. Charity, H. Han, K. Jing, M.A. MicMahan, R.J. McDonald, L.G. Moretro, G.J. Wozniak, W.L. Kehoe, B. Libby, and A.C. Mignerey, Proceerings of the 8th High Energy Heavy Ion Study, LBL - 24580, p. 352, (1987).

B. Borderie, M. Montoyz, M.F. Rivet, D. Jouan, C. Cabot, H. Fuchs, D. Gardes, H. Gauvin, D. Jacquet, and F. Monnet, Phys. Lett. 205B, 26 (1988).

U.L. Businaro and S. Gallone, Nuovo Cim. 1, 629, 1277 (1955).

X. Campi and S. Stringari, Z Phys. A309, 239 (1983).

N. Carjan and J.M. Alexander: Phys. Rev. C 38, 1692 (1988).

C. Cerruti, J. Desbois, R. Boisgard, C. Ngo, J. Natowitz, and J. Nemeth, Nucl. Phys. A476, 74 (1988). 
Ch 79 L.G. Christophorou, D.L. McCorkle, D.V. Maxey, and J.G. Carter, Nucl. Instr. Meth. 163, 141 (1979).

Ch 83a C.B. Chitwood, D.J. Fields, C.K. Gelbke, W.G. Lynch, A.D.

Panagiotou, M.B. Tsang, H. Utsunomiya, and W.A. Freidman, Phys. Lett. 131B, 289 (1983).

Ch 83b Y. Chan, M. Murphy, R.G. Stokstad, I. Tserruya, S. Wald, and A Buizzanowski, Phys. Rev. C 27, 447 (1983).

Ch 86 R.J. Charity, M.A. McMahan, D.R. Bowman, Z.H. Liu, R.J. McDonald, G.J. Wozniak, L.G. Moretto, S. Bradley, W.L. Kehoe, A.C. Mignerey, and M.N. Namboodiri, Phys. Rev. Lett. 56, 1354 (1986).

Ch 88a R.J. Charity, D.R. Bowman, Z.H. Liu, R.J. McDonald, M.A. McMahan, G.J. Wozniak, L.G. Moretto, S. Bradley, W.L. Kehoe, and A.C. Mignerey, Nucl. Phys. A476, 516 (1988).

Ch 88b R.J. Charity, M.A. McMahan, G.J. Wozniak, R.J. McDonald, L.G.Moretto, D.G. Sarantities, L.G. Sobotka, G. Guarino, A. Pantaleo, L. Fiore, A. Gobbi, and X.D. Hildenbrand, Nucl. Phys. A483, 371 (1988). Ch 89 R.J. Charity, K. Jing, D.R. Bowman, M.A. McMahan, G.J. Wozniak, L.G. Moretto, N, Colonna, G. Guarino, A. Panteleo, L. Fiore, A. Gobbi, and K. Hildenbrand, Lawrence Berkeley Laboratory preprint LBL-26859 and submitted to Nucl. Phys., (1989).

Co 89 N. Colonna, R.J. Charity, D.R. Bowman, M.A. McMahan, G.J. Wozniak, L.G. Moretto, G. Guarino, A. Panteleo, L. Fiore, A. Gobbi, and K.D. Hildenbrand, Phys. Rev. Lett. 62, 1833 (1989).

Cu 83 M.W. Curtin, H. Toki, and D.K. Scoth, Phys. Lett. 123B, 289, (1983).

De 85 K. Thomas R. Davies and Amold J. Sierk, Phys. Rev. C 31, 915 (1985).

Da 86 R. Dayras, A. Pagano, J. Barrette, B. Berthier, D.M. De Castro Rizzo, E. Chavez, O. Cisse, R. Legrain, M.C. Mermaz, E.C. Pollacco, H. 
Delagrange, W. Mittig, B. Heusch, R. Coniglione, G. Lanzano, and A. Palmeri, Nucl. Phys. A460, 299 (1986).

De $87 a$

De 87b

Du 84

Fa 87

Fi 67

Fi 71

Fi 82

Fi 84

Fi 89

Fr 83a

Fr 83b

Ga 75

Ge 80

Ga 82
J. Desbois, R. Boisgard, C. Ngo, and J. Nemeth, Z. Phys. A328, 101 (1987).

J. Desbois, Nucl. Phys. A466, 724, (1987).

W. Dunnweber, W. Hering, H. Puchta, R. Ritzka, W. Trautmann, W.

Trombik, and C. Egelhaaf, Phys. Rev. Lett. 52, 1405 (1984).

M. Fatyga, R.C. Byrd, K. Kwiatkowski, W.G. Wilson, L.W. Woo, V.E.

Viola, Jr., H.J. Karwowski, J. Jastrzebski, and W. Skulski, Phys. Lett.

185B, 321 (1987).

M.E. Fiseher, Physics (N.Y.), 3, 255 (1967).

M.E. Fischer, Proceedings of the International School of Physics. Enrico

Fermi, Course LI, Critical Phenomena, editor M.S. Green (Academic, New York, 1971).

J.E. Finn, S. Agarwal, A. Bujak, J. Chuang, L.J. Gutay, A.S. Hirsch, R.W. Minich, N.T. Porile, R.P. Scharenberg, B.C. Stringfellow, and F. Turkot, Phys. Rev. Lett. 49, 1321 (1982).

D.J. Fields, W.G. Lynch, C.B. Chitwood, C.K. Gelbke, M.B. Tsang, H. Utsunomiya, and J. Aichelin, Phys. Rev. C 30, 1912 (1984).

D.E. Fields, K. Kwiatkowski, D. Bonser, R.W. Viola, V.E. Viola, W.G. Lynch, J. Pochodzalla, M.B. Tsang, C.K. Gelbke, D.J. Fields, and Sam Austin, Phys. Lett. 220B, 356 (1989).

W.A. Friedman and W.G. Lynch, Phys. Rev. C 28, 16 (1983).

W.A. Freidman and W.G. Lynch, Phys. Rev. C 28, 950 (1983).

J. Galin, Nucl. Phys. A255, 472 (1975).

A. Gavron, Phys. Rev. C 21, 230 (1980).

J. Galin, H. Oeschler, S. Song, B. Borderie, M.F. Rivet, I. Forest, R. 
Bimbot, D. Gardes, B. Gatty, H. Guillemot, M. Lefort, B. Tamain, and X. Tarrago, Phys. Rev. Lett. 48, 1787 (1982).

Go 74

Go 77

Go 88

Gr 85

Gr 86

Gr 87a

Gr 87b

Gr 87c

Gr 88

Gu 82

Ha 52

Ha 87

$\mathrm{Ha} 89$

Hi 84

Hu 78

A.S. Goldhaber, Phys. Lett. 53B, 306 (1974).

J. Gosse:, H.H. Gutbrod, W.G. Meyer, A.M. Poskanzer, A. Sandoval, R. Stock, and G.D. Westfall, Phys. Rev. C 16, 629 (1977).

J. Gomez del Campo, J.L. Charvet, A. D'Onofrio, R.L. Auble, J.R. Beene, M.L. Halbert ,and H.J. Kim, Phys. Rev. Lett. 61, 290 (1988).

C. Gregoire, B. Remaud, F. Scheuter, and F. Sebille, Nucl. Phys. A436, 365 (1985).

D.H.E. Gross, X. Zhang, and S. Xu, Phys. Rev. Lett. 56, 1544 (1986).

D.H.E. Gross and H. Massman, Nucl. Phys. A471, 339c (1987).

C. Gregoire, B. Remaud, F. Sebrille, L. Vinet, and Y. Raffray, Nucl. Phys. A465, 317 (1987).

C. Gregoire, B. Remaud, F. Sebrille, and L. Vinet, Phys. Lett. 186B, 14 (1987).

D.H.E. Gross, Phys. Lett. 203B, 26 (1988).

Hans H. Gutbrod, A.I. Warwick, and H.H. Wieman, Nucl. Phys. A387, 177c (1982).

H. Hauser and H. Feshbach, Phys. Rev. 87, 366 (1952).

S. Harar, Nucl. Phys. A471, 205c (1987).

H.Y. Han, K.X. Jing, E. Plagnol, D.R. Bowman, R.J. Charity, L. Vinet, G.J. Wozniak, and L.G. Moretto, Nucl. Phys. A492 138 (1989).

A.S. Hirsch, A. Bujak, J.E. Finn, L.J. Gutay, R.W. Minich, N.T. Porile, R.P. Scharenberg, B.C. Stringfellow, and F. Tu.kot, Phys. Rev. C 29, 508 (1984).

F. Hubert, A. Fleury, R. Bimbot, and D. Gardes, Ann. de Phys. S, Suppl. (1980). 
Hy 71 Earl K. Hyde, Gilbert W. Butler, and A.M. Poskanzer, Phys. Rev. C 4, 1759 (1971).

Ja 83a B.V. Jacak, G.D. Westfall, C.K. Gelbke, L.H. Harwood, W.G. Lynch, D.K. Scott, H. Stocker, M.B. Tsang, and T.J.M. Symons, Phys. Rev. Lett. 51, 1846 (1983).

Ja 83b H.R. Jaqaman, A.Z. Mekjian, and L. Zamick, Phys. Rev. C 27, 2782 (1983).

Ja 84

H.R. Jaqaman, A.Z. Mekjian, and L. Zamick, Phys. Rev. C 29, 2067 (1984).

$\mathrm{Ka} 70$

S.B. Kaufman, B.D. Wilkins, M.J. Fluss, and E.P. Steinberg, Nucl. Instr. Meth. 82, 117 (1970).

Ke 89 W.L. Kehoe, Ph.D. Thesis, University of Maryland (1989).

Ko 73

Ralph G. Korteling, C.R. Toren, and Earl K. Hyde, Pinys. Rev. C 7, 1611 (1973).

$\mathrm{Kr} 84$

H.W. Kraner, Nucl. Instr. Meth. A225, 615 (1984).

$\mathrm{Kr} 85$

H. Kruse, B.V. Jacak, J.J. Molitoris, G.D. Westfall, and H. Stocker, Phys. Rev. C 31, 1770 (1985).

Li 88

J. Liu and H. Matis, Lawrence Berkeley Laboratory report PUB - 3071 (1988).

Lo 84 J.A. Lopez and P.J. Siemens, Nucl. Phys. A431, 728 (1984).

Ly 82 U. Lynen, H. Ho, W. Kuhn, D. Pelte, U. Winkler, W.F.J. Mueller, Y.-T. Chu, P. Doll, A. Gobbi, K. Hildenbrand, A. Olmi, H. Sann, H. Stelzer, R. Bock, H. Lohner, R. Glasow, and R. Santo, Nucl. Phys. A387, 129c (1982).

Mc 85 M.A. McMahan, L.G. Moretto, M.L. Padgett, G.J. Wozniak, L.G. Sobotka, and M.G. Mustafa, Phys. Rev. Lett. 54, 1995 (1985). M.A. McMahan, IEEE Transactions on Nuclear Science 35, 42 (1988). 
Me 80

Mi 53

Mi 82

Mo 72

Mo 75

Mo 78

Mo 80

Mo 81a

Mo 81b

Mo 84

Mo 85

Mo 86

Ne 86a

Ne 86b

$\mathrm{Pa} 84$
W.G. Meyer, H.H. Gutbrod, Ch. Lukner, and A. Sandoval, Phys. Rev. C 22, 179 (1980).

J.M. Miller and G. Friedlander, Phys. Rev. 91, 485 (1953).

R.W. Minich, S. Agarwal, A. Bujak, J. Chuang, J.E. Finn, L.J. Gutay, A.S. Hirsch, N.T. Porile, R.P. Scharenberg, B.C. Stringfellow, and F. Turkot, Phys. Lett. 118B, 458 (1982).

L.G. Moretto, Phys. Lett 40B, 185 (1972).

L.G. Moretto, Nucl. Phys A247, 211 (1975).

J.B. Moulton, J.E. Stephenson, R.P. Schmith, and G.J. Wozniak, Nucl. Instr. Meth. 157, 325 (1978).

L.G. Moretto and R.P. Schmitt, Phys. Rev. C 21, 204 (1980).

L.G. Moretto and R.P. Schmitt, Reports on Progress in Physics 44, 533 (1981).

D.J. Morrissey and L.G. Moretto, Phys. Rev. C 23, 1835 (1981).

H. Morgenstem, W. Bohne, W. Galster, K. Grabisch, and A. Kyanowski, Phys. Rev. Lett. 52, 1104 (1984).

J.J. Molitoris, D. Hahn, and H. Stocker, Progress in Particle and Nuclear Physics 15, 239 (1985).

L.G. Moretto and D.R. Bowman, Proceedings of the XXIV International Winter Meeting on Nuclear Physics, Bormio, Italy, ed. I. Iori, Ricerca Scientifica ed Educazione Permanente, Suppl. 49, p. 126 (1986). J. Nemeth, M. Barranco, J. Desbois, and C. Ngo, Z. Phys. A325, 347 (1986).

J. Nemeth, M. Barranco, C. Ngo, and E. Tomasi, Z. Phys. A323, 419 (1986).

A.D. Panagiotou, M.W. Curtin, H. Toki, D.K. Scott, and P.J. Siemens, Phys. Rev. Lett. 52, 496 (1984). 
Pa 85

Pl 89

Po 71

Ra 81

Sa 76

Sa 89

Sc 77

Sc 82

Si 84

Si 86

Sn 88

So 83

So 84

So 85

So 89

St 85
A.D. Panagiotou, M.W. Curtin, and D.K. Scott, Phys. Rev. C 31, 55 (1985).

E. Plagnol, L. Vinet, D.R. Bowman, Y.D. Chan, R.J. Charity, E. Chavez, S.B. Gazes, H. Han, W.L. Kehoe, M.A. McMahan, L.G. Moretto, R.G. Stokstad, G.J. Wozniak, and G. Auger, Phys. Lett. 221B, 11 (1989). A.M. Poskanzer, Gilbert W. Butler, and Earl K. Hyde, Phys. Rev. C 3, 882 (1971).

J. Randrup and S.E. Koonin, Nucl. Phys. A356, 223 (1981).

G. Sauer, H. Chandra, and U. Mosel, Nucl. Phys. A264, 221 (1976).

D.G. Sarantities, D.R. Bowman, G.J. Wozniak, R.J. Charity, Z.H. Liu, R.J. McDonald, M.A. McMahan, and L.G. Moretto, Phys. Lett. 218B, 427 (1989).

W.U. Schroder and J.R. Huizenga, Annual Review of Nuclear and Particle Science 27, 465 (1977).

R.P. Schmitt and A.J. Pacheco, Nucl. Phys. A379, 313 (1982).

P.J. Siemens, Nature 305, 410 (1984).

Amold J. Sierk, Phys. Rev. C 33, 2039 (1986).

K. Sneppin and L. Vinet, Nucl. Phys. A480, 342 (1988).

L.G. Sobotka, M.L. Padgett, G.J. Wozniak, G. Guarino, A.J. Pacheco, L.G. Moretto, Y. Chan, R.G. Stokstad, I. Tserruya, and S. Wald, Phys. Rev. Lett. 51, 2187 (1983).

L.G. Sobotka, M.A. McMahan, R.J. McDonald, C. Signarbieux, G.J. Wozniak, M.L. Padget, J.H. Gu, Z.H. Liu, ZQ. Yao, and L.G. Moretto, Phys. Rev. Lett. 53, 2004 (1984).

L.G. Sobotka and L.G. Moretto, Phys. Rev. C 31, 668 (1985).

L.G. Sobotka, private communication (1989).

G.S.F. Stephans, D.G. Kovar, R.V.F. Janssens, G. Rosner, H. Ikezoe, 
B. Wilkins, D. Henderson, K.T. Lesko, J.J. Kolata, C.K. Gelbke, B.V. Jacak, Z.M. Koenig, G.D. Westfall, A. Szanto de Toledo, E.M. Szanto, and P.L. Gonthier, Phys. Lett. 161B, 60 (1985).

Su 54 Nathan Sugarman, Robert B. Duffield, G. Friedlander, and J.M. Miller, Phys. Rev. 95, 1704 (1954).

Sw 76 W.J. Swiatecki, report (unpublished) (1976).

Sw 82 W.J. Swiatecki, Nucl. Phys. A376, 275 (1982).

To 85

J. Toke, R. Bock, G.X. Dai, A. Gobbi, S. Gralla, K.D. Hildenbrand, J. Kuzminski, W.F.J. Mueller, A. Olmi, and H. Stelzer, Nucl. Phys. A440, 327 (1985).

Tr 84

W. Trautmann, W. Dunnweber, W. Hering, C. Lauterbach, H. Puchta, R. Ritzka, and W. Trombik, Nucl. Phys. A422, 418 (1984).

$\operatorname{Tr} 85$ R. Trockel, K.D. Hildenbrand, U. Lynen, W.F.J. Mueller, H.J. Rabe, H. Sann, H. Steizer, R. Wada, N. Brummund, R. Glasow, K.H. Kampert, R. Santo, D. Pelte, J. Pochodzalla, and E. Eckert, Gesellschaft fur Schwerionenforschung Report 85 - 45, 1985.

$\operatorname{Tr} 88$ R. Trockel, K.D. Hildenbrand, U. Lynen, W.F.J. Muller, H.J. Rabe, H. Sann, H. Stelzer, W. Trautmann, R. Wada, E. Eckert, P. Kreutz, A. Kuhmichel, and J. Pochodzaila, Phys. Rev. C 39, 729 (1988).

Ts 88 M.B. Tsang, W.G. Lynch, R.M. Ronningen, Z. Chen, C.K. Gelbke, T. Nayak, J. Pochodzalla, F. Zhu, M. Tohyama, W. Trautmann, and W. Dunnweber, Phys. Rev. Lett. 60, 1479 (1988).

Va 73 Robert Vandenbosch and John R. Huizenga, Nuclear Fission, (Academic Press, New York, 1973).

Va 83 J. van der Plicht, H.C. Brith, M.M. Fowler, Z. Fraenkel, A. Gavron, J.B. Wilhelmy, F. Plasil, T.C. Awes, and G.R. Young, Phys. Rev. C 28, 2022 (1983). 
Vi 82

Vi 85

Wa 82

Wa 83

We 37

We 76

We 78

Wh 63

Wo 56

Wo 82

Ye 88
V.E. Viola, Jr., B.B. Back, K.L. Wolf, T.C. Awes, C.K. Gelbke, and H. Breuer, Phys. Rev. C 26, 178 (1982).

V.E. Viola, K. Kwiatkowski, and M. Walker, Phys. Rev C 31, 1550 (1985).

A.I. Warwick, A. Baden, H.H. Gutbrod, M.R. Maier, J. Peter, H.G.

Ritter, H. Stelzer, H.H. Wieman, F. Weik, M. Freedman, D.J. Henderson, S.B. Kaufman, E.P. Steinberg, and B.D. Wilkins, Phys. Rev. Lett. 48, 1719 (1982).

A.I. Warwick, H.H. Wieman, H.H. Gutbrod, M.R. Maier, J. Peter, H.G. Ritter, H. Stelzer, F. Weik, M. Freedman, D.J. Henderson, S.B. Kaufman, E.P. Steinberg, and B.D. Wilkins, Phys.Rev. C 27, 1083 (1983).

V.F. Weisskopf, Phys. Rev. 52, 295 (1937).

G.D. Westfall, J. Gosset, P.J. Johansen, A.M. Poskanzer, W.G. Meyer, H.H. Gutbrod, A. Sandoval, and R. Stock, Phys. Rev. Lett. 37, 1202 (1976).

G.D. Westfall, R.G. Sextro, A.M. Poskanzer, A.M. Zebelman, G.W. Butler, and E.K. Hyde, Phys. Rev. C 17, 1368 (1978).

J.A. Wheeler, East Neutron Physics, Vol. II (Interscience, New York, 1963) p. 2051.

R. Wolfgang, E.W. Baker, A.A. Caretto, J.B. Cumming, G. Friedlander, and J. Hudis, Phys. Rev. 103 (1956).

C.Y. Wong and K. Van Bibber, Phys. Rev. C 25, 2990 (1982).

S. Yennello, K. Kwiatkowski, D.E. Fields, R. Planeta, V.E. Viola, Y.

Cassagnou, R. Dayras, S. Harar, R. Legrain, E.C. Pollacco, C. Volant, and E. Hourani, Indiana University Nuclear Chemistry Report. INC. 40007-55, p. 31 (1988). 


\section{9}

Ze 75

A.M. Zebelman, A.M. Poskanzer, J.D. Bowman, R.G. Sextro, and V.E. Viola, Jr., Phys. Rev. C 11, 1280 (1975).

Zh 87a X. Zhang, D.H.E. Gross, S. Xu, and Y. Zheng, Nucl. Phys. A461, 641 (1987).

Zh 87b X. Zhang, D.H.E. Gross, S. Xu, and Y. Zheng. Yu-ming, Nucl. Phys. A461, 668 (1987). 